\title{
Bibliography of Work on the
} Heterogeneous Photocatalytic Removal of Hazardous Compounds from Water and Air Update Number 1 To June, 1995

Daniel M. Blake

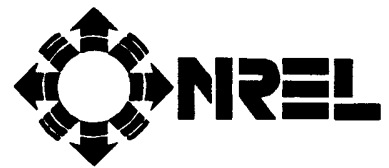

National Renewable Energy Laboratory 1617 Cole Boulevard Golden, Colorado 8040 1-3393 A national laboratory of the U.S. Department of Energy Managed by the Midwest Research Institute for the U.S. Department of Energy under Contract No. DE-AC36-83CH10093 


\section{NOTICE}

This report was prepared as an account of work sponsored by an agency of the United States government. Neither the United States government nor any agency thereof, nor any of their employees, makes any warranty, express or implied, or assumes any legal liability or responsibility for the accuracy, completeness, or usefulness of any information, apparatus, product, or process disclosed, or represents that its use would not infringe privately owned rights. Reference herein to any specific commercial product, process, or service by trade name, trademark, manufacturer, or otherwise does not necessarily constitute or imply its endorsement, recommendation, or favoring by the United States government or any agency thereof. The views and opinions of authors expressed herein do not necessarily state or reflect those of the United States government or any agency thereof.

Available to DOE and DOE contractors from:

Office of Scientific and Technical Information (OSTI)

P.O. Box 62

Oak Ridge, TN 37831

Prices available by calling (615) 576-8401

Available to the public from:

National Technical Information Service (NTIS)

U.S. Department of Commerce

5285 Port Royal Road

Springfield, VA 22161

(703) $487-4650$ 


\section{Summary}

The Solar Industrial Program, funded by the United States Department of Energy, is carrying out research and development on processes for the destruction or removal of hazardous substances from water and air. The work is being done at the National Renewable Energy Laboratory in Golden, CO, and Sandia National Laboratory in Albuquerque, NM, and by their subcontractors. The processes of interest in this report are based on the application of heterogeneous photocatalysts. The current state of the art in catalysts are forms of titanium dioxide or modifications thereof but work on other heterogeneous catalysts is included in this compilation.

This report is an update of a bibliography of work done on the photocatalytic oxidation of organic or inorganic compounds in air or water and on the photocatalytic reduction of metal containing ions in water that was published in May, 1994. The previous report included 663 citations obtained through the middle of 1993 and some selected references from the balance of that year. This update contains an additional 574 references. These were published during the period from January 1993 to June 1995, or are references from prior years that were not included in the initial report The general focus of the work is removing hazardous contaminants from air or water to meet environmental or health regulations. This report follows the same organization as the previous publication. The first part provides citations for work done in a few broad categories that are generic to the process. Three tables provide references to work on specific substances. The first table covers organic compounds that are included in various lists of hazardous substances identified by the United States Environmental Protection Agency (EPA). The second table lists compounds not included in those categories, but which have been treated in aphotocatalytic process. The third table covers inorganic compounds that are on EPA lists of hazardous materials or that have been treated by a photocatalytic process. A new section has been added which gives information about companies that are active in providing products based on photocatalytic processes or that can provide pilot, demonstration, or commercial-scale water- or air-treatment systems. Key words, assigned by the author of this report, have been included with the citations in the listing of the bibliography. 


\section{Table of Contents}

Introduction

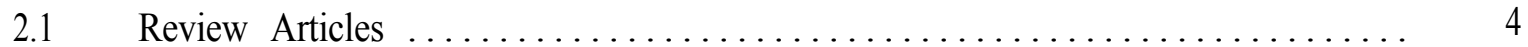

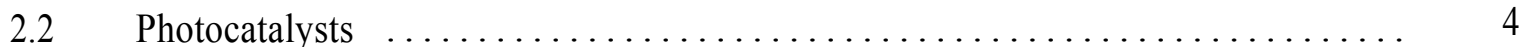

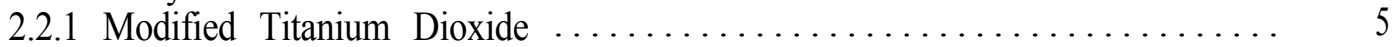

2.2.2 Hydrophobic Surface Treatment ........................ 5

2.2.3 Dye Sensitized Titanium Dioxide. ........................ 5

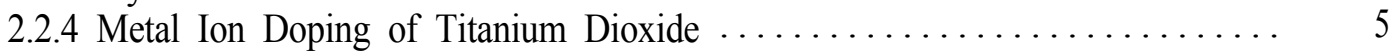

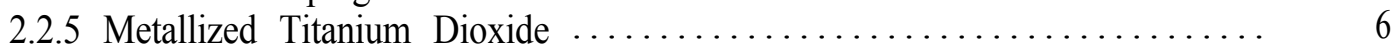

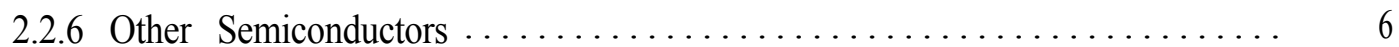

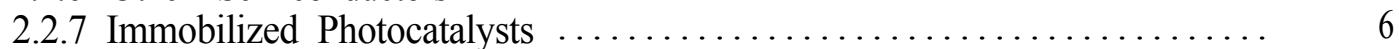

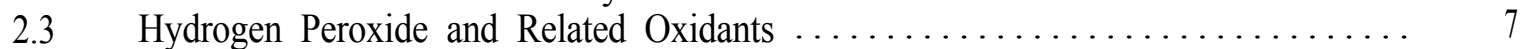

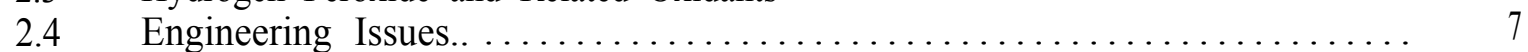

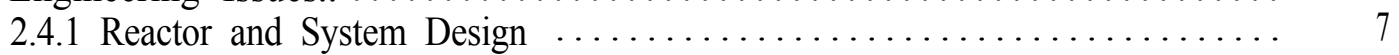

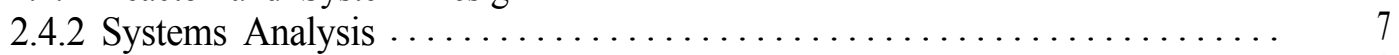

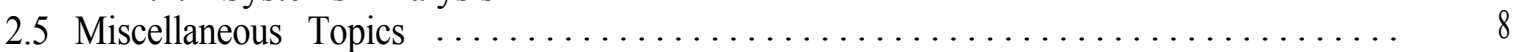

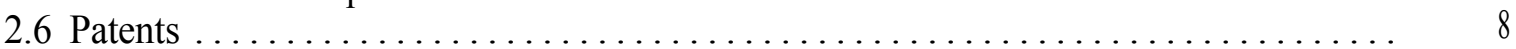

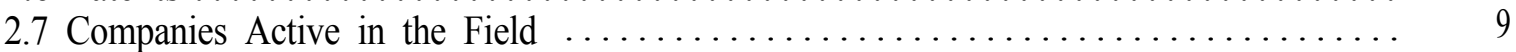

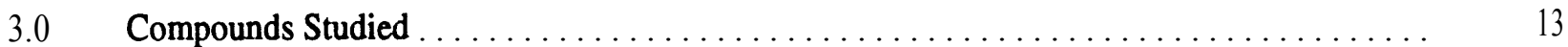

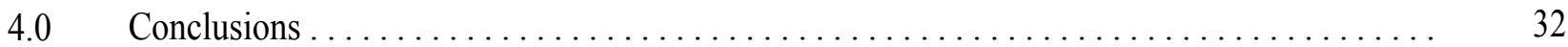

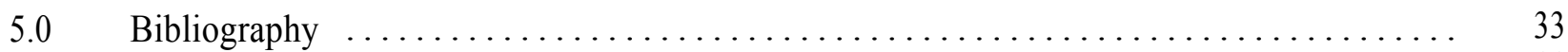

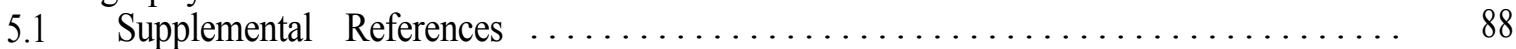

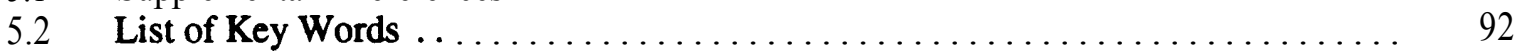

5.3 Address for Sending Corrections or Additions to the Bibliography $\ldots \ldots \ldots \ldots \ldots .92$

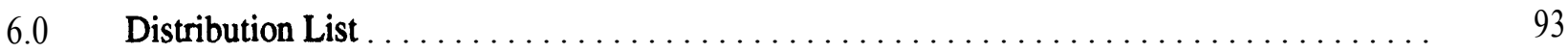




\subsection{Introduction}

This update in combination with the previous report provides a comprehensive bibliography of work available in the open literature for scientists and engineers interested in the use of heterogeneous photocatalytic oxidation or reduction processes in environmental remediation, process emission control, indoor air quality, or other applications. The combined bibliographies include more than 1200 citations to work published between 1970 and the middle of 1995. The literature cited includes United States and foreign patents. Information was compiled by manually scanning the literature and searching commercial databases. This update includes about 100 citations to work done prior to 1993 that were not included in the initial report. Some citations have doubtless been missed and topics covered in certain papers may not have been identified and covered in every appropriate category. The limitation to heterogeneous processes means that work on homogeneous photocatalytic processes is not included. Examples of these are the recently patented process based on the iron(III)-oxalate-hydrogen peroxide system ${ }^{2}$ and the systems based on soluble polyoxometalates. ${ }^{3}$ The author invites readers to send references to relevant work that appeared before 1995 that has been missed to the mail ur e:mail address included in Section 5.3 of this report.

The photocatalytic oxidation of organic compounds in water is the subject of a large body of research that has been performed in laboratories all over the world. A lesser but rapidly increasing amount of work has been devoted to the oxidation of volatile organic or inorganic compounds in the gas phase. Photocatalytic reduction of both organic compounds and metal-containing ions is also receiving increasing attention. Representative reactions are shown below:

'Blake, Daniel M. (1994). Bibliography of Work on the Photocatalytic Removal of Hazardous Compoundsfrom Water and Air. NREL/TP-430-6084. Golden, CO: National Renewable Energy Laboratory. 75 pp. [DE94006906] Available from the National Technical Information Service, Springfield, VA 22161.

${ }^{2}$ Safarzedeh-Amiri, Ali, inventor. "Photocatalytic Method for Treatment of Contaminated Water." Cryptonics Corporation, assignee. United States Patent, 5,266,214. November 30, 1993.

${ }^{3}$ Hill, C. L. and C. M. Prosser-McCartha. "Photocatalytic and Photoredox Properties of Polyoxometalate Systems." Photosensitization and Photocatalysis Using Inorganic and Organometallic Compounds, eds. K. Kalyanasunderam and M. Gratzel, 307-30, Dordrecht: Kluwer Academic Publishers, 1993. 


$$
\begin{gathered}
\mathrm{C}_{6} \mathrm{H}_{5} \mathrm{OH}_{(\mathrm{aq})}+7 \mathrm{O}_{2(\mathrm{aq})} \frac{\text { semiconductor }}{\mathrm{hv}} 6 \mathrm{CO},+3 \mathrm{H}_{2} \mathrm{O} \\
\mathrm{C}_{2} \mathrm{HCl}_{3(\mathrm{~g})}+3 / 2 \mathrm{O}_{2(\mathrm{~g})}+\mathrm{H}_{2} \mathrm{O}_{(\mathrm{g})} \frac{\text { semiconductor }}{\mathrm{hv}} 2 \mathrm{CO}_{2(\mathrm{~g})}+3 \mathrm{HCl}_{(\mathrm{g})} \\
2 \mathrm{CrO}_{4(\mathrm{aq})}^{-2}+\mathrm{CH}_{3} \mathrm{OH}_{(\mathrm{aq})}+4 \mathrm{H}+\underset{\mathrm{semiconductor}}{\longrightarrow} 2 \mathrm{Cr}(\mathrm{OH})_{3(\mathrm{~s})}+\mathrm{co},+\mathrm{H}_{2} \mathrm{O}
\end{gathered}
$$

Reactions are shown in the idealized case of complete oxidation or reduction. It is widely observed that intermediates and by-products are often formed that persist in the treated stream. These can include a variety of acids, oxygenated compounds, and carbon monoxide.

The net process involves oxidizing the organic compound to an intermediate stage of oxygen content or to carbon dioxide, water, and a mineral acid (if a heteroatom such as nitrogen or chlorine is present). Other oxidizing agents may be substituted for oxygen. Modifying or removing certain metal ions from water can be accomplished when the ions replace oxygen as the electron acceptor in the process and sacrificial electron donor compounds are provided.

A survey of the literature collected reveals that about $20 \%$ of the new citations are for work in which the target compound is carried in the gas phase rather than in water. Twenty-four citations cover work on disinfection of water or air or to potential medical applications. Control of odor compounds or application to removal of volatile organic compounds specifically for indoor air purification was the subject of nine papers. Use of the sun as the light source was identifiable in more than 80 papers. Engineering issues were addressed in more than 70 papers. Of the 46 patents that are included in the new list, 35 have assignees in Japan.

Reference to test work on more than 150 compounds is included in the Tables in Section 3. The most-studied compounds are phenol derivatives, BTEX (benzene, toluene, ethyl benzene, and xylene) components found in fuel spills, and chlorinated solvents such as trichloroethylene and chloroform. A significant number of pesticides, dye compounds, and surfactants have been shown to be mineralized and a variety of bacteria and virus have been found to be killed by irradiation with near-ultraviolet light in the presence of titanium dioxide. The breadth of work attests to the very wide range of applications that are being evaluated for the technology. 
A new section has been added which lists the names and addresses of companies that offer products or services based on a heterogeneous photocatalytic process. These are mainly in the United States and Canada but a few are from Europe and Japan. The author has attempted to obtain information directly from the company or used information from the open literature in the preparation of the entries which identify the product or service. Companies not listed are invited to send information, to the address in Section 5.3, that can be included in the next update of the bibliography.

The following sections cover reviews written on various aspects of the technology, work in developing and testing photocatalysts and oxidants, engineering issues, other topics, patents, and companies active in bringing photocatalytic processes or devices to the marketplace. These sections, which include information that can apply to a range of applications, are followed by tables listing references to work performed on specific substances. Documents referring to work on systems in which the compound to be treated is carried in the gas phase are indicated by the prefix " $\mathrm{g}$ " in the citation number. 


\subsection{Generic Information}

This section refers to work that cuts across the field of photocatalytic processes.

\subsection{Review Articles}

Many new reviews have been written that cover various aspects of photocatalytic chemistry and technology. This section also includes reviews written in the years prior to 1993 that were not cited in the first report. Reviews covering the broad topics given can be found in the following references:

methodology for comparing reaction systems $-21,350$, and 428; mechanisms/activity $\mathbf{- 2 7}, \mathbf{5 0}, \mathbf{6 3}$, $133,205,258,290,325,338,425,426,427,429,535,537,546,549,550,561$, and 569 ; ultra small particles - 28,206, 326, and 547; photoelectrochemical systems - 32 and 33; solar facilities - 36; photocatalysis - 45, 116, 124, 309, 366, 543, 549, 564, 568, and 570; solar processes - 51, 509, 531 , and 574; companies active in the field - 69; adsorption -90,91, and 537; metallized photocatalysts 145; disinfection - 150 and 319; indoor air quality - 150,319, and 565; oil spills - 150; surfactant degradation - 159 and 360; environmental applications -171, 266, 286, 300, 316, 342, 343, 358, 381, $421,422,424,425,429,517,556,558,559,560,562,565$, and 571 ; production and applications of ultra-fine $\mathrm{TiO}_{2}$ - 201; preparation and applications of semiconductor thin films - 204; microfabrication and integrated chemical systems - 226; overview of Department of Energy R\&D in photocatalysis - 289; Degussa P25 properties - 321; new photocatalytic processes - 338; titanium dioxide pigments and the control of photodegradation of polymers - 339; comparison of advanced oxidation processes - 340 and 557; issues and questions in application of photocatalysis - 341; solar production of fuels and chemicals - 381; and photochemical reactors - 390 .

\subsection{Photocatalysts}

The nature of the photocatalyst determines the rate and efficiency of the process. The anatase form of titanium dioxide has the desirable properties of being chemically stable, readily available, and active as a catalyst for oxidation processes. On the negative side, its $3.2 \mathrm{eV}$ band gap results in only a small overlap of its action spectrum with the solar spectrum. Also, the efficiency for converting photons absorbed to hazardous molecules destroyed is generally low, particularly for the aqueous phase processes. In order to identify ways to increase process efficiency and to improve the overlap of the absorption spectrum of the photocatalyst with 
the solar spectrum, a great deal of work has been done on modifying $\mathrm{TiO}_{2}$ and testing other semiconductors. This work is broken down into a few broad categories and covered in the references cited.

\subsubsection{Modified Titanium Dioxide}

Titanium dioxide and modified forms, including different commercially available forms, heat treated materials, and materials prepared by a range of techniques:

sol-gel $-11,167,211,212,355,376,520$, and 523 ; rutile $-12,46,48,160,167,219,220,292$, and 389 ; heat treatment $-22,49,78,167,303,337,352,353,406,520$, and 526 ; colloidal or quantized particles $-28,67,81,114,115,136,170,200,241,254,277,278,279,389,510,511,519$, and 548; modified - 29; xerogel/aerogel - 94, 95, 96,97, 230,413, and 466; flame synthesis - 119 and 376; or catalysts from different vendors - 405 .

\subsubsection{Hydrophobic Surface Treatment}

Titanium dioxide has been modified to make the surface hydrophobic to alter the interaction with organic compounds in water. This work is covered in the following: silica - 94.

\subsubsection{Dye Sensitized Titanium Dioxide}

Dye sensitizers have been used in conjunction with titanium dioxide to improve the response to visible light: ruthenium complex - 38, 200, and 460; eosin - 151; cobalt tetraphenylphorphine - 187; cobalt tetrasulfophthalocyanine - 235; organic dyes - 209 and 393; polyviologen - 397;

$\left[\mathrm{Mo}_{2} \mathrm{~S}_{4}\left(\mathrm{~S}_{2} \mathrm{C}_{2} \mathrm{H}_{4}\right)\right](-2)-254$; iron-porphyrin - g368; coupled semiconductors - 423; or

Ni-phthalocyanines -499 .

\subsubsection{Metal Ion Doping of Titanium Dioxide}

Other metal ions have been introduced into the titanium dioxide lattice to modify the properties. They are covered in the following:

$\mathrm{Si}-323 ; \mathrm{Al}-82$ and 323; V - 40, 81, 82, and 279; Fe - 28, 40, 47, 49, 81, 82, 259, 278, 311, and 348; $\mathrm{Co}-40$ and 82; $\mathrm{Zr}-230 ; \mathrm{Ni}-474 ; \mathrm{Nb}-89,223$, and 230; Mo - 81, 82, 242, 352, 353, and 380; W $104,105,269,270,352,353$, and 380; Re - 81 and 82; Ru - 81 and 82; Rh - 81 and 82; or Os - 81. 


\subsubsection{Metaiiized Titanium Dioxide}

Noble metals have been deposited on the titanium dioxide surface to enhance catalytic activity:

$\mathrm{Cu}$ - 311,504, and 515; $\mathrm{Ru}$ - 203; Os - 203; Rh - 203; Ir - 203; Pd -151, 203, 269, 270, 333, and 335;

$\mathrm{Ag}-243,269,270,311,337$, and 459; $\mathrm{Au}-31,244,269,270$, and 337; or Pt -23, 24, 87, 88, 146,

$147,160,164,191,203,269,270,273,317,327,337,370,401,413,467$, and 486 .

\subsubsection{Other Semiconductors}

A wide range of other semiconductors have been tested for photocatalytic activity. In general they have been found to be less active than titanium dioxide. Relevant work is cited in the following:

$\mathrm{Al}_{2} \mathrm{O}_{3}-148$ and g436; $\mathrm{Sr}-$ or $\mathrm{BaTiO}_{3}-219$ and 220; $\mathrm{V}_{2} \mathrm{O}_{5}-\mathrm{g} 14$ and g367; $\mathrm{Fe}_{2} \mathrm{O}_{3}-28,87,106,114$, $148,184,259,356,364,423,461,541$, and 542; $\mathrm{FeO}(\mathrm{OH})-135,354$, and 524; $\mathrm{CaFe}_{2} \mathrm{O}_{4}-282 ; \mathrm{ZnO}-$ $9,23,28,30,106,157,160,204,217,261,278,292,337,356,357,364, \mathrm{~g} 367,385,386,388,423$, g436, 461, g487, 499, 545, 566, and 573; Zn- or CdS - 106, 112, 143, 301, 337, 423, 433, 519,539, and 540; $\mathbf{Z O}_{2}-87$ and g367; $\mathbf{Z P O}_{4}-313 ; \mathbf{M o O}_{3}-\mathrm{g} 14 ; \mathrm{MoS}_{2}-160 ; \mathrm{SnO}_{2}-9,38,87,204,356, \mathrm{~g} 367$, 482, and 484; $\mathrm{Sb}_{2} \mathrm{O}_{4}-\mathrm{g} 367 ; \mathrm{CeO}_{2}-\mathrm{g} 367 ; \mathrm{WO},-9,39,106,160,204,337,356, \mathrm{~g} 367,499,541$, and 542; $\mathrm{SiO}_{2}-148, ; \mathrm{SiC}-221 ; \mathrm{Nb}_{2} \mathrm{O}_{5}-17 ; \mathrm{RuS}_{2}-18$; fly ash - 148 and 555; polyaniline - 296; or natural minerals - g192 and 354 .

\subsection{7 immobilized Photocatalysts}

Most experimental work on aqueous systems has been performed using the photocatalyst in the form of fine particles suspended in the liquid phase. In a waste treatment application it would be simpler if the catalyst were immobilized in the photoreactor so the material would not have to be separated from the treated fluid in a subsequent process step. For the treatment of gases most work has involved immobilized catalyst. Titanium dioxide has been affixed to a variety of surfaces:

glass (including fibers) - g14, 42, 152, 153, 172, 173, 174, 194, 212, 265, 280, 281, 304, 314, 315, 320,345, $\mathbf{g} 440, \mathbf{4 8 1}, \mathbf{4 8 4}, \mathbf{4 8 5}, \mathbf{5 5 2}, \mathbf{5 5 3}, \mathbf{5 5 4}$, and 572; silica - 186 and 532; metal - 178 and 446; ceramic-2, 10, g16, 168, 403, g407; clays - 260,449, and g452; polymer -1, 12, 40, 41, 60, 70, 145, $149,296,345,401$, and 474 ; thin films $-26,38,83,84,144,210,223,334,398,406$, and 523; zeolite $-14,35,120,143,260$, and 402; alumina - 143; carbon -179, 184, 185, 231, 312, g453, and 473; Ambersorb - 327; or $\boldsymbol{\beta}$-cyclodextrin - 510 . 


\subsection{Hydrogen Peroxide and Other Oxidants}

Oxygen has been the oxidant of choice in most studies, but hydrogen peroxide has been found to improve the rates of reaction with a variety of organic substrates under some conditions. This work is covered in the following:

hydrogen peroxide $-13,22,40,62,92,102,110,126,137,170,173,251,310,330,394$, and 399; persulfate - 130; or ozone -17 and g436.

\subsection{Engineering Issues}

In recent years the success of laboratory work has led to interest in applying the technology to environmental remediation and treatment of process waste streams. Work has appeared in the literature addressing issues related to the scale-up of the process and resolution of engineering problems. Progress has been significant and many companies are now providing turn-key systems for treating contaminated water and air (see Section 2.7).

\subsubsection{Reactor and System Design}

A number of papers have addressed topics relevant to the design of reactors for photocatalytic processes: photochemical reactors - 390; photoelectrochemical system - 10; non-concentrating reactor - 137, $138,330,347,421,471,513,514$, and 532; shallow ponds/tanks $-37,85$, and 512; parabolic trough $52,53,71,72,134,288,306$, and 346; kinetic modelling - 68, 214, 215, 276, 298, 301, 302, and 572; recirculating reactor -80 and 456 ; fixed bed $-88,110,369,415$, and 530; filtration - 110; hybrid lamp/solar system - 111; equipment requirements - 197 and 421; ultrasonic reactor - 199; batch-type reactors - 228 and 229; optimization of air purification systems - 363; controlled periodic illumination - 418,419, and 420; or falling film reactor - 521 .

\subsubsection{Systems Analysis}

As the technology for the photocatalytic treatment of contaminated air or water has progressed, a few studies have compared the costs of sunlight and electric lamps as photon sources; others have compared the photocatalytic processes with conventional treatment methods, such as carbon adsorption or W-peroxide oxidation: 
process waste water - 53, 394, and 442; photocatalysis process cost $-56,110,251,364,421,431$, g472, and 557; or photon costs from lamps and sunlight - 411 and 471 .

\subsection{Miscellaneous Topics}

This category includes papers of interest that do not fall into the preceding headings: adsorption - 382 and 528; combined photocatalytic and biological treatment -152, 227, 267, 268, 310, 412, 434,435, and 476; non-aqueous solvent systems - 488 and 49 1; or electron microscopy of $\mathrm{TiO}_{2}$ phases -493.

\subsection{Patents}

The number of patents that cover aspects of photocatalytic technology has increased rapidly in the last decade. They cover a range of aqueous and gas-phase applications. The general topic of the patents are included in the following:

catalyst formulation - 1, 149, 193, 224, 225, 280, 281, 312, 345, 400, 404, 502, and 518;

photoelectrochemical system - 10 and 344; spent plating bath - 24; point-of-use water treatment system - 65; water treatment system - 85 and 456; regeneration of adsorbents - 87; metals removal from water - 118 and 281 ; magnetic catalyst particle - 128; oil spill treatment - 152; catalyst on inorganic fibers - 168; photocatalysis with $185 \mathrm{~nm}$ light for air treatment - 169; removal of chlorinated compounds from vent gas (includes scrubber) - 183; removal of air pollutants (catalyst on exterior walls of buildings) - 185 and 335; additive to enhance degradation of plastics in the environment -

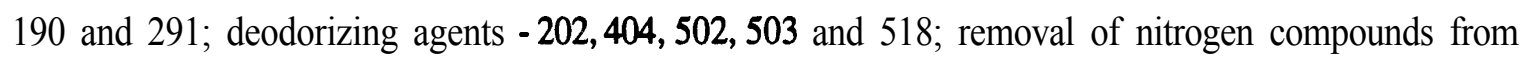
water - 222; regenerable deodorant - 231; combined biological and photocatalytic treatment of wastewater - 267 and 268; process for killing cells or disinfection - 283,391, and 503; photocatalyst plate material - 285; total organic carbon analyzer - 287; nitrogen and phosphorus analyzer - 451; purification of waste gases - 331; disinfecting construction materials - 333; methanol synthesis - 334 and 345; fluid purification - 391; method for preparing dialkyldipyridinium salts - 395; semiconductor modified with insoluble polyviologen derivatives - 396; moisture-resistant catalyst for ethylene oxidation - 400; or optical fiber as light source -489 . 


\subsection{Companies Active in the Field}

The following is a list of companies that have products or services based on heterogeneous photocatalysis. The list is compiled from the best information available to the author from personal contacts and material published in the open literature.

American Energy Technologies, Inc.

Mr. Greg Peebles

P.O. Box 1865

Green Cove Springs, FL 32043

Telephone: 904-284-0552

Fax: 904-284-0006

Product(s): Stable W-transmissive polymers and glass for photoreactor construction and development and fabrication of photoreactors.

Degussa Corporation

Ms. Maria Nargiello

3500 Embassy Parkway, Suite 100

Akron, OH 44333

Telephone: 2 16-668-2235

Fax: $216-668-3846$

Product(s): Photocatalysts, fumed titanium dioxide, P25 and high surface area P25.

Industrial Solar Technology

Mr. Ken May

4420 McIntyre Street

Golden, CO 80403

Telephone: $303-279-8108$

Fax: 303-279-8107

-Product(s): Compound parabolic concentrators, parabolic concentrators, and glass and polymer tubes for solar reactors.

Ishihara Sangyo Kaishi, Ltd.

1-3-11, Edohori, Nishi-ku

Osaka City 550, JAPAN

Telephone: +81-6-444-5812

Fax: $+81-6-444-5878$

Product(s): Photocatalytic paints and papers.

IT Corporation

Mr. Richard Miller

312 Directors Drive

Knoxville, TN 37923

Telephone: 423-690-3211

Fax: 423-694-9573

Product(s): Provides technology based solutions to environmental problems, reactor design and process engineering for treatment systems, and manufactures treatment systems through its NEPCCO Equipment Division. 
Kato Manufacturing Company, Ltd.

400, Iwasaki, Komaki City

Aichi Pref. 485, JAPAN

Telephone: $\mathbf{+ 8 1 - 5 6 8 - 7 2 - 8 2 8 0}$

Fax: +81-568-75-1385

Product(s): Glassware with photocatalytic titanium dioxide coatings.

KSE, Inc.

Dr. Charles Quinlan

P.O. Box 368

Amherst, MA 01004

Telephone: 413-549-5506

Fax: 413-549-5788

Product(s): Development and manufacturing of air emission control equipment for environmental remediation, industrial emissions, and indoor air quality applications.

\section{LightStream Photocatalytic LLC}

(A Division of E. Heller \& Company)

Ephraim Heller

1311 Harbor Bay Parkway

Suite 1000

Alameda, CA 94502

Telephone: 5 10-748-690 1

Fax: 5 10-748-6902

Product(s): Photocatalytic indoor air purification systems and photocatalytic paints and coatings.

Matrix Photocatalytic, Inc.

Mr. Bob Henderson

22 Pegler Street

London, Ontario

CANADA N5Z 2B5

Telephone: 5 19-660-8669

Fax: 5 19-660-8525

Product(s): Develop and supply photocatalytic treatment systems for aqueous and gas phase environmental remediation and control of process emissions.

\section{NEPCCO}

Mr. John S. "Sandy" Reese

2140-100 N.E. 36th Avenue

Ocala, FL 34470

Telephone: 904-867-7482

Fax: 904-867- 1320

Product(s): Provides photocatalytic oxidation treatment systems as stand alone units or integrated into existing treatment or manufacturing processes.

Photo-Catalytics, Inc.

Mr. Gerald Cooper

755 S. 42nd Street

Boulder, CO 80303

Telephone: $303-494-7623$

Fax: $303-494-7623$ 
Photox

Dr. Elliot Berman

P.O. Box 15717

Kenmore Station

Boston, MA 02215

Telephone: 617-353-6407

Fax: 617-353-6466

Product(s): Development of photocatalysts and coatings for indoor air purification and residential water treatment.

Purifics Environmental Technologies, Inc.

Mr. Brian Butters

161 Mallard Road

London, Ontario

CANADA NOM 120

Telephone: $519-473-5788$

Fax: 519-473-0934

Product(s): Sell or lease turn-key $\mathrm{TiO}_{2}$ photocatalytic systems for treatment of ground water, process, and ultra pure water, perform laboratory and on-site treatability tests, and perform design work to integrate units into existing treatment systems.

Sachtleben Chemie

Z. Hd. Bernhard Becker/NPP

Dr.-Rudolf-Sachtleben - Str. 4

D-47198 Duisburg, GERMANY

Telephone: 02066-22-0 Fax: 02066-2222-00

Product(s): Photocatalysts, sulfate process titanium dioxide, Hombikat UV 100

Science Applications International

Mr. Kelly Beninga

15000 West 6th Avenue

Suite 202

Golden, CO 80401

Telephone: 303-279-5677

Fax: 303-384-0320

Product(s): Develop and fabricate low-cost photochemical reactors.

Solar Kinetics, Inc.

Mr. Bennett Howell

P.O. Box 540636

Dallas, TX 75354

Telephone: 2 14-556-2376

Fax: 214-869-4158

Product(s): Develop and demonstrate solar photocatalytic systems for water treatment.

Trojan Technologies

Dr. William Cairns or Mr. Rory Murphy

3020 Gore Road

London, Ontario

CANADA N5V $4 \mathrm{~T} 7$

Telephone: 5 19-457-3400

Fax: $519-457-3030$

Product(s): Cooperative and in-house research and development of commercial photocatalytic systems. 
United Technologies Research Center

Dr. James Freihaut

411 Silver Lane, MS 129-24

East Hartford, CT 06108

Telephone: 203-727-7328

Fax: 203-727-2 151

Product(s): Research and development services for photocatalytic systems. 


\subsection{Compounds Studied}

The tables in this section have the same format as in the first report. No compounds have been removed from the tables but new compounds have been added to the second and third tables to incorporate the expanded work. The list of the compounds included in various lists of priority pollutants, air toxics, and the toxic release inventory compiled by the EPA ${ }^{4}$ provides a convenient frame of reference for citing the application of photocatalysis to compound oxidation. Table 1 lists compounds in the EPA categories; Table 2 lists organic compounds that are not in EPA lists; and Table 3 covers inorganic compounds in EPA lists or that have been treated by a photocatalytic process. The inorganic compounds are arranged by element unless a significant number of citations referred to work on a specific ion or compound. Formulas of compounds, when given, are not in the standard format because the software used to prepare the tables does not support subscripts. A few broad categories are included in Table 2 that reflect new applications: bacteria, algae, and virus; coal or carbon; adsorbable organic halides (AOX); color or chemical oxygen demand (COD); and oil or petroleum. Again, the citation prefix " $\mathrm{g}$ " indicates a gas-phase study. The treatability of compounds not demonstrated can in many cases be inferred from results for related compounds in the tables.

\footnotetext{
4"Notice of the Second Priority List of Hazardous Substances Commonly Found at Superfund Sites," Environmental Reporter, October 28, 1988, 1255-1260.
} 
Table 1. Organic Compounds in EPA Lists of Priority Pollutants, Air Toxics, or Toxic Release Inventory

\begin{tabular}{|c|c|c|c|c|}
\hline Substance & Formula & $\begin{array}{l}\text { Halo- } \\
\mathrm{g} \mathrm{e} \mathrm{n} \\
\end{array}$ & $\begin{array}{c}\text { Het. } \\
\text { Atom } \\
\end{array}$ & Reference \\
\hline 1,1,1-Trichloroethane & $\mathrm{C}: \mathrm{HCl} 2 \mathrm{CH} 2 \mathrm{Cl}$ & $\mathrm{Cl}$ & & $; 433,232,275$ \\
\hline 1.1,2,2-Tetrachloroethane & $\mathrm{C} \cdot \mathrm{HCl} 2 \mathrm{CHCl} 2$ & $\mathrm{Cl}$ & & $: 43,275$ \\
\hline 1, I.2-Trichloroethane & $\mathrm{C}: \mathrm{HCl} 2 \mathrm{CH} 2 \mathrm{Cl}$ & $\mathrm{Cl}$ & & $: 75, g 384$ \\
\hline 1,1,2-Trichloro-1,2.2-trifluoroethane & $\mathrm{C}=\mathrm{Cl} 2 \mathrm{FCClF} 2$ & Cl.F & & \\
\hline 1.1 -Dichloroethane & $\mathrm{C}=\mathrm{H} 3 \mathrm{CHCl} 2$ & $\mathrm{Cl}$ & & $: 75$ \\
\hline 1,1 -Dimethyl hydrazine & $(\mathrm{CH} 3) 2 \mathrm{NNH} 2$ & & $\mathrm{~N}$ & \\
\hline 1,2,3-Trichloropropane & $\mathrm{C} \cdot \mathrm{H} 2 \mathrm{ClCHClCH} 2 \mathrm{Cl}$ & $\mathrm{Cl}$ & & \\
\hline 1,2.4-Trichlorobenzene & $\mathrm{C}=6 \mathrm{H} 3 \mathrm{Cl} 3$ & $\mathrm{cl}$ & & 159,474 \\
\hline 1.2,4-Trimethylbenzene & $\mathrm{C} 6 \mathrm{H} 3(\mathrm{CH} 3) 3$ & & & \\
\hline 1,2-Butylene oxide & $\mathrm{H} 2 \mathrm{COCHCH} 2 \mathrm{CH} 3$ & & & \\
\hline 1,2-Dibromoethane & $\mathrm{B} 3 \mathrm{rCH} 2 \mathrm{CH} 2 \mathrm{Br}$ & $\mathrm{Br}$ & & \\
\hline 1,2-Dibromo-3-chloropropane (DBCP) & $\mathrm{C}=\mathrm{H} 2 \mathrm{BrCHBrCH} 2 \mathrm{Cl}$ & $\mathrm{Br}, \mathrm{Cl}$ & & \\
\hline 1,2-Dichlorobenzene & $\mathrm{C} 6 \mathrm{H} 4 \mathrm{Cl} 2$ & $\mathrm{Cl}$ & & \\
\hline 1.2-Dichloroethane & $\mathrm{ClCH} 2 \mathrm{CH} 2 \mathrm{Cl}$ & $\mathrm{Cl}$ & & $6,232,275$ \\
\hline 1,2-Dichloroethylene & $\mathrm{ClHC}: \mathrm{CHCl}$ & $\mathrm{Cl}$ & & \\
\hline 1,2-Dichloropropane & $\mathrm{CH} 3 \mathrm{CHClCH} 2 \mathrm{Cl}$ & $\mathrm{cl}$ & & $: 32$ \\
\hline 1,2-Dinitrotoluene & $\mathrm{C} 6 \mathrm{H} 3 \mathrm{CH} 3(\mathrm{NO} 2) 2$ & & $\mathbf{N}$ & \\
\hline 1.2-Diphenylhydrazine & $\mathrm{C} 12 \mathrm{H} 12 \mathrm{~N} 2$ & & $\mathrm{~N}$ & \\
\hline 1,2-Trans-dichloroethene & $\mathrm{C} 2 \mathrm{H} 2 \mathrm{Cl} 2$ & $\mathrm{Cl}$ & & \\
\hline 1.3.5-Trinitrobenzene & $\mathrm{C} 6 \mathrm{H} 3(\mathrm{NO} 2) 3$ & & $\mathrm{~N}$ & \\
\hline 1,3-Butadiene & $\mathrm{H} 2 \mathrm{C}: \mathrm{CHHC}: \mathrm{CH} 2$ & & & \\
\hline 1,3-Dichlorobenzene & $\mathrm{C} 6 \mathrm{H} 4 \mathrm{Cl}$ & $\mathrm{Cl}$ & & \\
\hline 1,3-Dichloropropene & $\mathrm{CHCl}: \mathrm{CHCH} 2 \mathrm{Cl}$ & $\mathrm{Cl}$ & & 232 \\
\hline 1,4-Dichlorobenzene & $\mathrm{C} 6 \mathrm{H} 4 \mathrm{Cl} 2$ & $\mathrm{Cl}$ & & $38,89,104,242,243,244,380$ \\
\hline 1.4-Dioxane & $\mathrm{OCH} 2 \mathrm{CH} 2 \mathrm{OCH} 2 \mathrm{CH} 2$ & & & \\
\hline 1 -Amino-2-methylanthrquinone & $\mathrm{C} 6 \mathrm{H} 4[\mathrm{C}(\mathrm{O})] 2 \mathrm{C} 6 \mathrm{H} 2 \mathrm{NH} 2 \mathrm{CH} 3$ & & & \\
\hline 1-Bromo-4-phenyoxybenzene & p-BrC6H4OC6H5 & $\mathrm{Br}$ & & \\
\hline 2,2,4-Trimethylpentane & $(\mathrm{CH} 3) 3 \mathrm{C} 5 \mathrm{H} 9$ & & & \\
\hline 2,3,7,8-Tetrachlorodibenzo-p-dioxin & $\mathrm{C} 12 \mathrm{H} 4 \mathrm{Cl} 4 \mathrm{O} 2$ & $\mathrm{Cl}$ & & \\
\hline 2,4,5-Trichlorophenoxyacetic acid & $\mathrm{C} 6 \mathrm{H} 2 \mathrm{Cl} 3 \mathrm{OCH} 2 \mathrm{CO} 2 \mathrm{H}$ & $\mathrm{Cl}$ & & \\
\hline 2,4.5-TP acid (silvex) & $\mathrm{Cl} 3 \mathrm{C} 6 \mathrm{H} 2 \mathrm{OCH}(\mathrm{CH} 3) \mathrm{COOH}$ & $\mathrm{Cl}$ & & \\
\hline 2,4,5-Trichlorophenol & $\mathrm{C} 6 \mathrm{H} 2 \mathrm{Cl} 3 \mathrm{OH}$ & $\mathrm{Cl}$ & & \\
\hline 2,4,6-Trichlorophenol & $\mathrm{C} 6 \mathrm{H} 2 \mathrm{Cl} 3 \mathrm{OH}$ & $\mathrm{Cl}$ & & 457 \\
\hline 2,4,6-Trinitrotoluene & $\mathrm{CH} 3 \mathrm{C} 6 \mathrm{H} 2(\mathrm{NO} 2) 3$ & & & 412,500 \\
\hline 2.4 Diaminoanisole & (NH2)2C6H3OCH3 & & $\mathrm{N}$ & \\
\hline 2,4-Dichlorophenoxyacetic acid (24-D) & $\mathrm{Cl} 2 \mathrm{C} 6 \mathrm{H} 3 \mathrm{OCH} 2 \mathrm{COOH}$ & $\mathrm{Cl}$ & & 380,467 \\
\hline 2.4-Diaminoanisole sulfate & (NH2)2C6H3OCH3.H2SO4 & & $\mathrm{N}$ & \\
\hline 2,4-Dichlorophenol & $\mathrm{Cl} 2 \mathrm{C} 6 \mathrm{H} 3 \mathrm{OH}$ & $\mathrm{Cl}$ & & $5,430,448,467,468$ \\
\hline 2,4-Dimethylphenol & $(\mathrm{CH} 3) 2 \mathrm{C} 6 \mathrm{H} 3 \mathrm{OH}$ & & & \\
\hline
\end{tabular}




\begin{tabular}{|c|c|c|c|c|}
\hline Substance & Formula & g & $\begin{array}{l}\text { Het. } \\
\text { Atom }\end{array}$ & Reference \\
\hline 2,4-Dinitrophenol & $\mathrm{C} 6 \mathrm{H} 3 \mathrm{OH}(\mathrm{NO} 2) 2$ & & $\mathrm{~N}$ & \\
\hline 2,4-Dinitrotoluene & $\mathrm{C} 6 \mathrm{H} 3 \mathrm{CH} 3(\mathrm{NO} 2) 2$ & & & \\
\hline 2,4-Toluene diamine & $\mathrm{CH} 3(\mathrm{NH} 2) 2 \mathrm{C} 6 \mathrm{H} 3$ & & $\mathbf{N}$ & \\
\hline 2,6-Dinitrotoluene & ( $66 \mathrm{H} 3 \mathrm{CH} 3(\mathrm{NO} 2) 2$ & & $\mathrm{~N}$ & \\
\hline 2,6-Xylidine & $(\mathrm{CH} 3) 2 \mathrm{C} 6 \mathrm{H} 3 \mathrm{NH} 2$ & & $\mathbf{N}$ & \\
\hline 2-Acetylaminofluorene & , $\mathrm{CH} 3 \mathrm{C}(\mathrm{O}) \mathrm{NHC} 6 \mathrm{H} 3 \mathrm{CH} 2 \mathrm{C} 6 \mathrm{H} 4$ & $\mathrm{~F}$ & $\mathrm{~N}$ & \\
\hline 2-Aminoanthraquinone & $\mathrm{C} 6 \mathrm{H} 4(\mathrm{CO}) 2 \mathrm{C} 6 \mathrm{H} 3 \mathrm{NH} 2$ & & $\mathbf{N}$ & \\
\hline 2-Butanone & $\mathrm{CH} 3 \mathrm{COCH} 2 \mathrm{CH} 3$ & & & \\
\hline 2-Chloroacetophenone & $\mathrm{C} 6 \mathrm{H} 5 \mathrm{COCH} 2 \mathrm{Cl}$ & $\mathrm{Cl}$ & & \\
\hline 2-Chloroethyl vinyl ether & $\mathrm{CH} 2 \mathrm{ClCH} 2 \mathrm{OCHCH} 2$ & $\mathrm{Cl}$ & & \\
\hline 2-Chlorophenol & $\mathrm{C} 6 \mathrm{H} 4 \mathrm{OHCl}$ & $\mathrm{Cl}$ & & $91,107,175,175,176,296,423,497$ \\
\hline 2-Ethoxyethanol & $\mathrm{H} 3 \mathrm{CCH} 2 \mathrm{OCH} 2 \mathrm{CH} 2 \mathrm{CH} 2 \mathrm{OH}$ & & & \\
\hline 2-Methoxyethanol & $\mathrm{MeOCH} 2 \mathrm{CH} 2 \mathrm{OH}$ & & & \\
\hline 2-Methylnaphthalene & $\mathrm{ClOH} 7 \mathrm{CH} 3$ & & & \\
\hline 2-Nitrophenol & $\mathrm{NO} 2 \mathrm{C} 6 \mathrm{H} 4 \mathrm{OH}$ & & $\mathrm{N}$ & \\
\hline 2-Nitropropane & $\mathrm{CH} 3 \mathrm{CHNO} 2 \mathrm{CH} 3$ & & $\mathrm{~N}$ & \\
\hline 2-Pentanone. 4-Methyl & $\mathrm{CH} 3(\mathrm{CH} 2) 2 \mathrm{COCH} 3$ & & & \\
\hline 2-Phenylphenol & $\mathrm{C} 6 \mathrm{H} 5 \mathrm{C} 6 \mathrm{H} 4 \mathrm{OH}$ & & & \\
\hline 3,3'-Dichlorobenzidine & $\mathrm{C} 6 \mathrm{H} 3 \mathrm{ClNH} 2 \mathrm{C} 6 \mathrm{H} 3 \mathrm{ClNH} 2$ & $\mathrm{Cl}$ & $\mathrm{N}$ & \\
\hline 3,3'-Dimethoxybenzidine & {$[\mathrm{C} 6 \mathrm{H} 3(\mathrm{OCH} 3) \mathrm{NH} 2] 2$} & & $\mathrm{~N}$ & \\
\hline 3.3'-Dimethylbenzidine (0-Tolidine) & {$[\mathrm{C} 6 \mathrm{H} 3(\mathrm{CH} 3) \mathrm{NH} 2] 2$} & & $\mathrm{~N}$ & \\
\hline 4.4'-Dichlorodiphenyldichloroethylene & $(\mathrm{ClC} 6 \mathrm{H} 4) 2 \mathrm{CCCl} 2$ & $\mathrm{Cl}$ & & \\
\hline 4,4'-Diaminodiphenyl ether & $\mathrm{NH} 29 \mathrm{C} 6 \mathrm{H} 4) 2 \mathrm{NH} 2$ & & $\mathrm{~N}$ & \\
\hline 4,4'-Isopropylidenediphenol & $(\mathrm{CH} 3) 2 \mathrm{C}(\mathrm{C} 6 \mathrm{H} 4 \mathrm{OH}) 2$ & & & \\
\hline $\begin{array}{l}\text { 4,4'-Methylenebis }(\mathrm{N}, \mathrm{N} \text {-dimethyl) } \\
\text { benzenamine }\end{array}$ & $\mathrm{C} 17 \mathrm{H} 22 \mathrm{~N} 2$ & & $\mathrm{~N}$ & \\
\hline 4,4'-Methylenedianiline & $\mathrm{H} 2 \mathrm{NC} 6 \mathrm{H} 4 \mathrm{CH} 2 \mathrm{C} 6 \mathrm{H} 4 \mathrm{NH} 2$ & & $\mathrm{~N}$ & \\
\hline 4,4'-Methylene-bis-(2-chloroaniline) & $\mathrm{CH} 2(\mathrm{C} 6 \mathrm{H} 4 \mathrm{ClNH} 2) 2$ & $\mathrm{Cl}$ & $\mathrm{N}$ & \\
\hline 4,4'-Thiodianiline & $\mathrm{C} 12 \mathrm{H} 12 \mathrm{~N} 2 \mathrm{~S}$ & & $\mathrm{~S}, \mathrm{~N}$ & \\
\hline 4,6-Dinitro-o-cresol & $\mathrm{CH} 3 \mathrm{C} 6 \mathrm{H} 2(\mathrm{NO} 2) 2 \mathrm{OH}$ & & $\mathrm{N}$ & \\
\hline 4,6-Dinitro-2-methylphenol & $\mathrm{C} 7 \mathrm{H} 6 \mathrm{~N} 2 \mathrm{O}$ & & $\mathrm{N}$ & \\
\hline 4-Aminoazobenzene & C6H5NNC6H4NH2 & & $\mathrm{N}$ & \\
\hline 4-Aminobiphenyl & C6H5C6H4NH2 & & $\mathrm{N}$ & \\
\hline 4-Chloroaniline & $\mathrm{ClC} 6 \mathrm{H} 4 \mathrm{NH} 2$ & $\mathrm{Cl}$ & & \\
\hline 4-Chlorophenyl phenyl ether & p-ClC6H4OC6H5 & $\mathrm{Cl}$ & & \\
\hline 4-Dimethylaminoazobenzene & (CH3)2C6H3NH2 & & $\mathrm{N}$ & \\
\hline 4-Methylphenol & $\mathrm{p}-\mathrm{CH} 3 \mathrm{C} 6 \mathrm{H} 4 \mathrm{OH}$ & & & 328 \\
\hline 4-Nitrobiphenyl & $\mathrm{C} 6 \mathrm{H} 5 \mathrm{C} 6 \mathrm{H} 4 \mathrm{NO} 2$ & & $\mathrm{~N}$ & \\
\hline 4-Nitrophenol & $\mathrm{NO} 2 \mathrm{C} 6 \mathrm{H} 4 \mathrm{OH}$ & & $\mathrm{N}$ & 140,348 \\
\hline 5-Nitro-0-anisidine & $\mathrm{NO} 2 \mathrm{C} 6 \mathrm{H} 3(\mathrm{NH} 2)(\mathrm{OCH} 3)$ & & $\mathrm{N}$ & \\
\hline Acenaphthene & $\mathrm{ClOH} 6(\mathrm{CH} 2) 2$ & & & \\
\hline Acenaphthylene & $\mathrm{Cl} 2 \mathrm{H8}$ & & & \\
\hline Acetaldehyde & $\mathrm{CH} 3 \mathrm{CHO}$ & & & $g 404, g 440$ \\
\hline
\end{tabular}




\begin{tabular}{|c|c|c|c|c|}
\hline Substance & Formula & $\begin{array}{l}\text { Halo- } \\
\text { gen }\end{array}$ & $\begin{array}{l}\text { Het. } \\
\text { Atom }\end{array}$ & Reference \\
\hline Acetamide & $\mathrm{CH} 3 \mathrm{CNOH} 2$ & & $\mathrm{~N}$ & 194 \\
\hline Acetone & $\mathrm{CH} 3 \mathrm{COCH} 3$ & & & $\begin{array}{l}\mathrm{g} 43 . \mathrm{g} 253 . \mathrm{g} 383 . \mathrm{g} 384 . \mathrm{g} 407, \\
\mathrm{~g} 450\end{array}$ \\
\hline Acetonitrile & $\mathrm{CH} 3 \mathrm{CN}$ & & $N$ & $\mathrm{~g} 43, \mathrm{~g} 252 . \mathrm{g} 253$ \\
\hline Acetophenone & $\mathrm{CH} 3 \mathrm{C}(\mathrm{O}) \mathrm{C} 6 \mathrm{H} 5$ & & & 477 \\
\hline Acrolein & $\mathrm{CH} 2 \mathrm{CHCHO}$ & & & \\
\hline Acrylamide & $\mathrm{CH} 2 \mathrm{CHCONH} 2$ & & $\mathrm{~N}$ & \\
\hline Acrylic acid & $\mathrm{H} 2 \mathrm{C}: \mathrm{CHCOOH}$ & & & \\
\hline Acrylonitrile & $\mathrm{H} 2 \mathrm{C}: \mathrm{CHCN}$ & & $\mathrm{N}$ & \\
\hline Aldrin & $\mathrm{C} 12 \mathrm{H} 8 \mathrm{Cl} 6$ & $\mathrm{Cl}$ & & \\
\hline Ally1 chloride & $\mathrm{H} 2 \mathrm{C}=\mathrm{CHCH} 2 \mathrm{Cl}$ & $\mathrm{Cl}$ & & \\
\hline Aniline & C6H5NH2 & & $N$ & 374 \\
\hline Anthracene & $\mathrm{C} 6 \mathrm{H} 4(\mathrm{CH}) 2 \mathrm{C} 6 \mathrm{H} 4$ & & & 98.188 \\
\hline Aramite & $\begin{array}{l}(\mathrm{CH} 3) 3 \mathrm{CC} 6 \mathrm{H} 4 \mathrm{OCH} 2 \mathrm{CH}(\mathrm{CH} 3)- \\
\mathrm{SO} 3 \mathrm{C} 2 \mathrm{H} 4 \mathrm{Cl}\end{array}$ & $\mathrm{cl}$ & $S$ & \\
\hline Atrazine & $\mathrm{C} 18 \mathrm{H} 14 \mathrm{ClN} 5$ & $\mathrm{Cl}$ & $\mathrm{N}$ & $80,296,355.356 .357,447,527$ \\
\hline Benzal chloride & $\mathrm{C} 6 \mathrm{H} 5 \mathrm{CHCl} 2$ & $\mathrm{Cl}$ & & \\
\hline Benzamide & C6H5CONH2 & & $\mathbf{N}$ & 271.272 \\
\hline Benzene & $\mathrm{C} 6 \mathrm{H} 6$ & & & $\begin{array}{l}\mathrm{g} 43,110,137,138,154, \mathrm{~g} 169.200, \\
269,322,330, \mathrm{~g} 384,414,470, \\
488.530\end{array}$ \\
\hline Benzidine & NH2(C6H4)2NH2 & & $N$ & \\
\hline Benzoic acid & $\mathrm{C} 6 \mathrm{H} 5 \mathrm{COOH}$ & & & 91,135 \\
\hline Benzoic tnchloride & $\mathrm{C} 6 \mathrm{H} 5 \mathrm{CCl} 3$ & $\mathrm{Cl}$ & & \\
\hline Benzoyl chloride & $\mathrm{C} 6 \mathrm{H} 5 \mathrm{COCl}$ & $\mathrm{Cl}$ & & \\
\hline Benzoyl peroxide & $(\mathrm{C} 6 \mathrm{H} 5 \mathrm{CO}) 2 \mathrm{O} 2$ & & & \\
\hline Benzo(a)anthracene & $\mathrm{C} 22 \mathrm{H} 14$ & & & 188 \\
\hline Benzo(a)pyrene & $\mathrm{C} 2 \mathrm{OH} 12$ & & & 188 \\
\hline Benzo(b)fluoranthene & $\mathrm{C} 2 \mathrm{OH} 12$ & & & \\
\hline Benzo(g,h.i) perylene & $\mathrm{C} 22 \mathrm{H} 12$ & & & \\
\hline Benzyl alcohol & $\mathrm{C} 6 \mathrm{H} 5 \mathrm{CH} 2 \mathrm{OH}$ & & & 91.120 \\
\hline Benzyl chloride & $\mathrm{C} 6 \mathrm{H} 5 \mathrm{CH} 2 \mathrm{Cl}$ & $\mathrm{Cl}$ & & \\
\hline BHC (Benzenehexachloride) & $\mathrm{C} 6 \mathrm{H} 6 \mathrm{Cl} 6$ & $\mathrm{Cl}$ & & \\
\hline Biphenyl & $\mathrm{C} 6 \mathrm{H} 5 \mathrm{C} 6 \mathrm{H} 5$ & & & \\
\hline bis(2-Chloroethoxy)methane & $\mathrm{CH} 2(2-\mathrm{ClC} 2 \mathrm{H} 5 \mathrm{O}) 2$ & $\mathrm{Cl}$ & & \\
\hline Bis(2-chloroethy!) ether & $\mathrm{ClCH} 2 \mathrm{CH} 2 \mathrm{OCH} 2 \mathrm{CH} 2 \mathrm{Cl}$ & $\mathrm{Cl}$ & & \\
\hline Bis(2-chloro- 1 -methylethyl) ether & {$[\mathrm{ClCH} 2(\mathrm{CH} 3) \mathrm{CH}] 2 \mathrm{O}$} & $\mathrm{Cl}$ & & \\
\hline Bis(2-ethylhexyl) adipate & $\mathrm{C} 7 \mathrm{H} 13) 2 \mathrm{C} 4 \mathrm{H} 8(\mathrm{CO} 2) 2$ & & & \\
\hline Bis(2-ethylhexyl)phthalate & $\mathrm{C} 4 \mathrm{H} 9 \mathrm{CH}(\mathrm{CH} 2)) 2 \mathrm{OOC}$ & & & \\
\hline Bis(chloromethyl)ether & $\mathrm{CH} 2 \mathrm{Cl}) \mathrm{O}(\mathrm{CH} 2 \mathrm{Cl})$ & $\mathrm{Cl}$ & & \\
\hline Bromochloromethane & $\mathrm{BrCH} 2 \mathrm{Cl}$ & $\mathrm{Br}, \mathrm{Cl}$ & & \\
\hline Bromodichloromethane & ב $\mathrm{HCl} 2 \mathrm{Br}$ & $\mathrm{Cl}, \mathrm{Br}$ & & \\
\hline Bromoethane & $22 \mathrm{H} 5 \mathrm{Br}$ & $\mathrm{Br}$ & & \\
\hline
\end{tabular}




\begin{tabular}{|c|c|c|c|c|}
\hline Substance & Formula & $\begin{array}{l}\text { Halo- } \\
\text { g e n }\end{array}$ & $\begin{array}{l}\text { Het. } \\
\text { Atom }\end{array}$ & Reference \\
\hline Bromoform (Tribromomethane) & $\mathrm{CHBr} 3$ & $\mathrm{Br}$ & & 235 \\
\hline Bromomethane (Methyl bromide) & $\mathrm{CH} 3 \mathrm{Br}$ & $\mathrm{Br}$ & & \\
\hline Butyl acrylate & $\mathrm{CH} 2: \mathrm{CHCOOC} 4 \mathrm{H} 9$ & & & \\
\hline Butylbenzyl phthalate & $\mathrm{C} 4 \mathrm{H} 900 \mathrm{OC} 6 \mathrm{H} 4 \mathrm{COOC} 7 \mathrm{H} 7$ & & & \\
\hline Butyraldehyde & $\mathrm{CH} 3(\mathrm{CH} 2) 2 \mathrm{CHO}$ & & & \\
\hline Calcium cyanamide & $\mathrm{NCNCa}$ & & $\mathrm{N}$ & \\
\hline Caprolactam & $\mathrm{CH} 2(\mathrm{CH} 2) 4 \mathrm{NHCO}$ & & $\mathrm{N}$ & \\
\hline $\begin{array}{l}\text { Captan (N-Trichloromethylmercapto- } \\
\text { tetrahydrophthalimide) }\end{array}$ & $\mathrm{C} 9 \mathrm{H} 8 \mathrm{Cl} 3 \mathrm{NO} 2 \mathrm{~S}$ & $\mathrm{Cl}$ & N.S & \\
\hline $\begin{array}{l}\text { Carbaryl [ } 1 \text {-Naphthalenol, } \\
\text { methylcarbamate] }\end{array}$ & $\mathrm{C} 10 \mathrm{H} 700 \mathrm{CNHCH} 3$ & & $\mathrm{~N}$ & 362 \\
\hline Carbon disulfide & $\operatorname{cs} 2$ & & S & \\
\hline Carbon tetrachloride & $\mathrm{CCl} 4$ & $\mathrm{Cl}$ & & $g 43,81,82.88 .164 .9169 .277$ \\
\hline Carbonyl sulfide & $\cos$ & & & \\
\hline Catechol & $\mathrm{C} 6 \mathrm{H} 4(\mathrm{OH}) 2$ & & & \\
\hline $\begin{array}{l}\text { Chloramben (Benzoic acid, 3-amino-2, } \\
\text { 5-dichloro-) }\end{array}$ & $\mathrm{C} 6 \mathrm{H}(\mathrm{CO} 2 \mathrm{H})(\mathrm{NH} 2) \mathrm{Cl} 2$ & $\mathrm{Cl}$ & $\mathrm{N}$ & \\
\hline Chlordane & c $10 \mathrm{H} 6 \mathrm{Cl} 8$ & $\mathrm{Cl}$ & & \\
\hline Chloroacetic acid & $\mathrm{CH} 2 \mathrm{ClCOOH}$ & $\mathrm{Cl}$ & & 40,275 \\
\hline Chlorobenzene & $\mathrm{C} 6 \mathrm{H} 5 \mathrm{Cl}$ & $\mathrm{Cl}$ & & $\mathrm{g} 102,322.336$ \\
\hline $\begin{array}{l}\text { Chlorobenzilnte (Benzeneacetic acid, } \\
\text { 4-chloro-alpha-(4-chlorophenyl)-) }\end{array}$ & $(\mathrm{C} 6 \mathrm{H} 4 \mathrm{Cl}) 2 \mathrm{C}(\mathrm{OH}) \mathrm{COOC} 2 \mathrm{H} 5$ & $\mathrm{Cl}$ & & \\
\hline Chlorodibentodioxins, various & c $1202 \mathrm{H} 8-\mathrm{xClx}$ & $\mathrm{Cl}$ & & \\
\hline Chlorodibenzofurans & c $120 \mathrm{H} 8-\mathrm{xClx}$ & $\mathrm{Cl}$ & & \\
\hline Chlorodibromomethane & $\mathrm{ClBr} 2 \mathrm{CH}$ & $\mathrm{Br}, \mathrm{Cl}$ & & \\
\hline Chlorodifluoromethane & CHClF2 & $\mathrm{Cl}, \mathrm{F}$ & & \\
\hline Chloroethane & $\mathrm{C} 2 \mathrm{H} 5 \mathrm{Cl}$ & $\mathrm{Cl}$ & & \\
\hline Chloroform & $\mathrm{CHCl} 3$ & $\mathrm{Cl}$ & & $\begin{array}{l}\mathrm{g} 43,73,81,129.219,220,232, \mathrm{~g} 253 \\
277,278,314,315,327,364\end{array}$ \\
\hline Chloromethane & $\mathrm{CH} 3 \mathrm{Cl}$ & $\mathrm{Cl}$ & & g263 \\
\hline Chloromethyl methyl ether & $\mathrm{C} 2 \mathrm{H} 5 \mathrm{ClO}$ & $\mathrm{Cl}$ & & \\
\hline Chloroprene & $\mathrm{H} 2 \mathrm{C}: \mathrm{CHCCl}: \mathrm{CH} 2$ & $\mathrm{Cl}$ & & \\
\hline $\begin{array}{l}\text { Chlorothalonil (1,3-Benzendedicarboni- } \\
\text { trile, 2.4.5,6-tetrachloro-) }\end{array}$ & $\mathrm{C} 6 \mathrm{Cl} 4(\mathrm{CN}) 2$ & & $\mathrm{~N}$ & \\
\hline Chrysene & $\mathrm{C} 18 \mathrm{H} 12$ & & & \\
\hline cis-1,2-Dichloroethylene & $\mathrm{ClHC}: \mathrm{CHCl}$ & $\mathrm{Cl}$ & & \\
\hline cis-1,3-Dichloropropene & $\mathrm{CHCl}: \mathrm{CHCH} 2 \mathrm{Cl}$ & $\mathrm{Cl}$ & & \\
\hline o-,m-.p-Cresols & $\mathrm{CH} 3 \mathrm{C} 6 \mathrm{H} 4 \mathrm{OH}$ & & & $60,61,429$ \\
\hline Cumene & $\mathrm{C} 6 \mathrm{H} 5 \mathrm{CH}(\mathrm{CH} 3) 2$ & & & \\
\hline Cumene hydroperox:de & $\mathrm{C} 6 \mathrm{H} 5 \mathrm{C}(\mathrm{CH} 3) 2 \mathrm{OOH}$ & & & \\
\hline $\begin{array}{l}\text { Cupferron (Benzeneamine, } \mathrm{N} \text {-hydroxy- } \\
\text { N-nitrose. ammori:m salt) }\end{array}$ & C6H5N(NO)ONH4 & & $\mathrm{N}$ & \\
\hline Cyclohexane & $\mathrm{C} 6 \mathrm{H} 12$ & & & \\
\hline
\end{tabular}




\begin{tabular}{|c|c|c|c|c|}
\hline Substance & Formula & $\begin{array}{l}\text { Halo- } \\
\text { gen }\end{array}$ & $\begin{array}{l}\text { Het. } \\
\text { Atom }\end{array}$ & Reference \\
\hline Cyclohexanone & $\mathrm{C} 6 \mathrm{H} 100$ & & & \\
\hline Cyclonite (RDX) & $(\mathrm{CH} 2) 4\left(\mathrm{NNO}_{2}\right) 4$ & & $\mathrm{~N}$ & \\
\hline Decabromodiphenyl oxide & $(\mathrm{C} 6 \mathrm{Br} 5) 2 \mathrm{O}$ & $\mathrm{Br}$ & & \\
\hline $\begin{array}{l}\text { Dialate [Carbamothioic acid. bis } \\
\text { (1-methylethyl)-, S-(2,3- } \\
\text { dichloro-2-propenyl) ester] }\end{array}$ & $[(\mathrm{CH} 3) 2 \mathrm{CH}] 2 \mathrm{NCOSCH} 2 \mathrm{CClCHC}]$ & $\mathrm{Cl}$ & N.S & \\
\hline Diaminotoluene (mixed isomers) & $\mathrm{CH} 3 \mathrm{C} 6 \mathrm{H} 3(\mathrm{NH} 2) 2$ & & $\mathrm{~N}$ & \\
\hline Diazomethane & $\mathrm{CH} 2 \mathrm{~N} 2$ & & $\mathrm{~N}$ & \\
\hline Dibenzofuran & $\mathrm{Cl} 2 \mathrm{H} 8 \mathrm{O}$ & & & \\
\hline Dibenzo(a.h)anthracene & $\mathrm{C} 22 \mathrm{H} 14$ & & & \\
\hline Dibromochloropropane & $\mathrm{CH} 2 \mathrm{BrCHBrCH} 2 \mathrm{Cl}$ & $\mathrm{Br}, \mathrm{Cl}$ & & \\
\hline Dibutyl phthalate & $\mathrm{C} 6 \mathrm{H} 4(\mathrm{COOC} 4 \mathrm{H} 9) 2$ & & & \\
\hline Dichlorobenzene (mixed isomers) & $\mathrm{C} 6 \mathrm{H} 4 \mathrm{Cl} 2$ & $\mathrm{Cl}$ & & \\
\hline Dichlorobromomethane & $\mathrm{CHBrC12}$ & $\mathrm{Cl}, \mathrm{Br}$ & & \\
\hline Dichlorodifluoromethane & $\mathrm{CCl} 2 \mathrm{~F} 2$ & $\mathrm{Cl}, \mathrm{F}$ & & \\
\hline $\begin{array}{l}\text { Dichlorvos (Phosphoric acid. } 2 \\
\text { dichloroethenyl dimethyl ester) }\end{array}$ & $(\mathrm{CH} 3 \mathrm{O}) 2 \mathrm{P}(\mathrm{O}) \mathrm{OCH}: \mathrm{CCl} 2$ & $\mathrm{Cl}$ & $\mathrm{P}$ & 265 \\
\hline $\begin{array}{l}\text { Dicofol ,4.4'-Dichloro-alpha-trichloro- } \\
\text { methylbenzhydroi }\end{array}$ & $\mathrm{C} 14 \mathrm{H} 9 \mathrm{Cl} 5 \mathrm{O}$ & $\mathrm{Cl}$ & & \\
\hline Dieldrin/aldrin & $\mathrm{C} 12 \mathrm{H} 10 \mathrm{OPCl} 6$ & $\mathrm{Cl}$ & $\mathrm{P}$ & \\
\hline Diepoxybutane & $\mathrm{C} 4 \mathrm{H} 6 \mathrm{O} 2$ & & & \\
\hline Diethanolamine & $(\mathrm{HOCH} 2 \mathrm{CH} 2) 2 \mathrm{NH}$ & & $\mathrm{N}$ & \\
\hline Diethyl phthalate & $\mathrm{C} 6 \mathrm{H} 4(\mathrm{CO} 2 \mathrm{C} 2 \mathrm{H} 5) 2$ & & & \\
\hline Diethyl sulfate & (C2H5)2SO4 & & $S$ & \\
\hline Dimethyl aminoazobenzene & $\mathrm{C} 6 \mathrm{H} 5 \mathrm{NNC} 6 \mathrm{H} 4 \mathrm{~N}(\mathrm{CH} 3)_{2}$ & & $\mathrm{~N}$ & \\
\hline Dimethyl formamide (DMF) & $\mathrm{HCON}(\mathrm{CH} 3) 2$ & & $\mathrm{~N}$ & \\
\hline Dimethyl phthalate & $\mathrm{C} 6 \mathrm{H} 4(\mathrm{COOCH} 3) 2$ & & & \\
\hline Dimethyl sulfate & $(\mathrm{CH} 3) 2 \mathrm{SO} 4$ & & $S$ & \\
\hline Dimethylcarbamyl chloride & $(\mathrm{CH} 3) 2 \mathrm{NCOCl}$ & $\mathrm{Cl}$ & $\mathrm{N}$ & \\
\hline Disulfoton & $(\mathrm{C} 2 \mathrm{H} 5 \mathrm{O}) 2 \mathrm{P}(\mathrm{S}) \mathrm{SCH} 2 \mathrm{CH} 2 \mathrm{SCH} 2 \mathrm{CH} 3$ & & P.S & \\
\hline Di-n-butyl phthalate & $\mathrm{C} 6 \mathrm{H} 4(\mathrm{COOC} 4 \mathrm{H} 9) 2$ & & & \\
\hline Di-n-octyl phthalate & $\mathrm{C} 6 \mathrm{H} 4(\mathrm{CO} 2)(\mathrm{n}-\mathrm{C} 8 \mathrm{H} 17) 2$ & & & \\
\hline Di-(2-ethylhexy) phthalate (DEHP) & $\mathrm{C} 6 \mathrm{H} 4[\mathrm{COOCH} 2 \mathrm{CH}(\mathrm{C} 2 \mathrm{H} 5) \mathrm{C} 4 \mathrm{H} 9] 2$ & & & \\
\hline Endosulfan & $\mathrm{C} 9 \mathrm{H} 6 \mathrm{Cl} 603 \mathrm{~S}$ & $\mathrm{Cl}$ & & \\
\hline Endrin aldehyde/ endrin & $(\mathrm{C} 12 \mathrm{H} 8 \mathrm{OCl})$ & $\mathrm{Cl}$ & & \\
\hline Epichlorohydrin & $\mathrm{CH} 2 \mathrm{OCHCH} 2 \mathrm{Cl}$ & $\mathrm{Cl}$ & & \\
\hline Ethyl acrylate & $\mathrm{CH} 2: \mathrm{CHCOOC2H5}$ & & & \\
\hline Ethyl chloroformate & $\mathrm{ClCOOC2H5}$ & $\mathrm{Cl}$ & & \\
\hline Ethylbenzene & $\mathrm{C} 6 \mathrm{H} 5 \mathrm{C} 2 \mathrm{H} 5$ & & & $35,137,138,330,477,530$ \\
\hline Ethylene & $\mathrm{H} 2 \mathrm{C}: \mathrm{CH} 2$ & & & $g 169, g 400, g 405, g 518$ \\
\hline Ethylene glycol & $\mathrm{CH} 2 \mathrm{OHCH} 2 \mathrm{OH}$ & & & 31 \\
\hline Ethylene oxide & $\mathrm{CH} 2 \mathrm{CH} 2 \mathrm{O}$ & & & g169 \\
\hline Ethylene thiourea & $\mathrm{NHCH} 2 \mathrm{CH} 2 \mathrm{NHCS}$ & & N.S & \\
\hline
\end{tabular}




\begin{tabular}{|c|c|c|c|c|}
\hline Substance & Formula & $\begin{array}{l}\text { Halo- } \\
\text { gen }\end{array}$ & $\begin{array}{l}\text { Het. } \\
\text { Atom }\end{array}$ & Reference \\
\hline Ethyleneimme ( Aziridine) & $\mathrm{CH} 2 \mathrm{NHCH} 2$ & & $\mathrm{~N}$ & \\
\hline $\begin{array}{l}\text { Fluometuron [Urea. N,N-dimethyl-N'-[3- } \\
\text { (trifluoromethyl)phenyl]-] }\end{array}$ & $\mathrm{ClOH} 11 \mathrm{~F} 3 \mathrm{~N} 2 \mathrm{O}$ & $\mathrm{F}$ & $\mathrm{N}$ & \\
\hline Fluoranthene & $\mathrm{C} 16 \mathrm{H} 10$ & & & \\
\hline Fluorene & $\mathrm{C} 6 \mathrm{H} 4 \mathrm{CH} 2 \mathrm{C} 6 \mathrm{H} 4$ & & & 98 \\
\hline Fluorotrichloromethane & $\mathrm{CCl} 3 \mathrm{~F}$ & Cl.F & & \\
\hline Formaldehyde & $\mathrm{HCHO}$ & & & g329,354 \\
\hline Heptachlor/heptachlor epoxide & $\mathrm{C} 10 \mathrm{H} 7 \mathrm{Cl} 7$ & $\mathrm{Cl}$ & & \\
\hline Heptane & $\mathrm{CH} 3(\mathrm{CH} 2) 5 \mathrm{CH} 3$ & & & g368 \\
\hline Hexachlorobenzene & $\mathrm{C} 6 \mathrm{Cl} 6$ & $\mathrm{Cl}$ & & 234 \\
\hline Hexachlorobutadiene & $\mathrm{Cl} 2 \mathrm{C}: \mathrm{CClCCl}: \mathrm{CCl} 2$ & $\mathrm{Cl}$ & & \\
\hline Hexachlorocyclopentadiene & $\mathrm{C} 5 \mathrm{Cl} 6$ & $\mathrm{Cl}$ & & \\
\hline Hexachloroethane & $\mathrm{Cl} 3 \mathrm{CCCl} 3$ & $\mathrm{Cl}$ & & \\
\hline Hexachloronaphthalene & $\mathrm{C} 10 \mathrm{H} 2 \mathrm{Cl} 6$ & $\mathrm{Cl}$ & & \\
\hline Hexamethylphosphoramide & {$[(\mathrm{N}(\mathrm{CH} 3) 2] 3 \mathrm{PO}$} & & $\mathrm{P}, \mathrm{N}$ & \\
\hline Hexamethylene- 1,6-diisocyanate & $\mathrm{OCN}(\mathrm{CH} 2) 6 \mathrm{NCO}$ & & $\mathrm{N}$ & \\
\hline Hexane & $\mathrm{CH} 3(\mathrm{CH} 2) 4 \mathrm{CH} 3$ & & & g43 \\
\hline Hydroqunone & $\mathrm{C} 6 \mathrm{H} 4(\mathrm{OH}) 2$ & & & 467521 \\
\hline Indeno( $1,2.3-c d)$ pyrene & $\mathrm{C} 22 \mathrm{H} 12$ & & & \\
\hline Isophorone & $\mathrm{C}(\mathrm{O}) \mathrm{CHC}(\mathrm{CH} 3) \mathrm{CH} 2 \mathrm{C}(\mathrm{CH} 3) 2 \mathrm{CH} 2$ & & & \\
\hline Isopropyl alcohol & $(\mathrm{CH} 3) 2 \mathrm{CHOH}$ & & & $28, \mathrm{~g} 46,115,143.203 .235 .386$ \\
\hline Lindane (gamma-Benzenehexachloride) & $\mathrm{C} 6 \mathrm{H} 6 \mathrm{Cl} 6$ & $\mathrm{Cl}$ & & \\
\hline Malachite Green & $\mathrm{C} 23 \mathrm{H} 25 \mathrm{CIN} 2$ & $\mathrm{Cl}$ & N & \\
\hline Malathion & $\begin{array}{l}(\mathrm{CH} 3 \mathrm{O}) 2 \mathrm{P}(\mathrm{S}) \mathrm{SCH}(\mathrm{CO} 2 \mathrm{C} 2 \mathrm{H} 5)- \\
\mathrm{CH} 2 \mathrm{CO} 2 \mathrm{C} 2 \mathrm{H} 5\end{array}$ & & P,S & 131,177 \\
\hline Maleic anhydride & $\mathrm{HC}: \mathrm{CHC}(\mathrm{O}) \mathrm{OC}(\mathrm{O})$ & & & 30 \\
\hline $\begin{array}{l}\text { Maneb (Carbamodithioic acid, 1,2- } \\
\text { ethanediylbis-,manganese complex) }\end{array}$ & (SSCNCH2CH2NHCSS)Mn & & N.S & \\
\hline Mechlorethamine & $\mathrm{CH} 3 \mathrm{~N}(\mathrm{CH} 2 \mathrm{CH} 2 \mathrm{Cl}) 2$ & $\mathrm{Cl}$ & $\mathrm{N}$ & \\
\hline Melamine & $\mathrm{H} 2 \mathrm{NCNC}(\mathrm{NH} 2) \mathrm{NC}(\mathrm{NH} 2) \mathrm{N}$ & & $\mathrm{N}$ & \\
\hline Methanol & $\mathrm{CH} 3 \mathrm{OH}$ & & & $\begin{array}{l}\mathrm{g} 43,53,203, \mathrm{~g} 252, \mathrm{~g} 253,293 \\
364,373, \mathrm{~g} 486,498\end{array}$ \\
\hline Methoxychlor & $\mathrm{Cl} 3 \mathrm{CCH}(\mathrm{C} 6 \mathrm{H} 4 \mathrm{OCH} 3) 2$ & $\mathrm{Cl}$ & & \\
\hline Methyl acrylate & $\mathrm{CH} 2: \mathrm{CHCOOCH} 3$ & & & \\
\hline Methyl butyl ketone & $\mathrm{CH} 3 \mathrm{COC} 4 \mathrm{H} 9$ & & & \\
\hline Methyl ethyl ketone & $\mathrm{CH} 3 \mathrm{COCH} 2 \mathrm{CH} 3$ & & & 88,448 \\
\hline Methyl iodide & $\mathrm{CH} 3 \mathrm{I}$ & I & & \\
\hline Methyl isobutyl ketone & $(\mathrm{CH} 3) 2 \mathrm{CHCOCH} 3$ & & & \\
\hline Methyl isocyanate & CH3NCO & & $\mathrm{N}$ & \\
\hline Methyl methacrylate & $\mathrm{CH} 2: \mathrm{C}(\mathrm{CH} 3) \mathrm{COOCH} 3$ & & & \\
\hline Methyl ten-butyl ether & $(\mathrm{CH} 3) 3 \mathrm{COCH}$ & & & $34,8383, g 334$ \\
\hline Methylene bromide & $\mathrm{CH} 2 \mathrm{Br} 2$ & $\mathrm{Br}$ & & \\
\hline Methylene chloride & $\mathrm{CH} 2 \mathrm{Cl} 2$ & $\mathrm{Cl}$ & & $40, g 43,232, \mathrm{~g} 253$ \\
\hline
\end{tabular}




\begin{tabular}{|c|c|c|c|c|}
\hline Substance & Formula & $\begin{array}{l}\text { Halo- } \\
\text { gen }\end{array}$ & $\begin{array}{l}\text { Het. } \\
\text { Atom }\end{array}$ & Reference \\
\hline Methylenebis(phenylisocyanate) (MBI) & $\mathrm{CH} 2(\mathrm{C} 6 \mathrm{H} 4 \mathrm{NCO}) 2$ & & $\mathrm{~N}$ & \\
\hline Methylhydrazine & $\mathrm{CH} 3 \mathrm{NHNH} 2$ & & $\mathrm{~N}$ & \\
\hline Michler's ketone & $\mathrm{CO}\left[\mathrm{C} 6 \mathrm{H} 4 \mathrm{~N}(\mathrm{CH} 3)_{2}\right] 2$ & & $\mathrm{~N}$ & \\
\hline Mirex & $\mathrm{ClOCl12}$ & $\mathrm{Cl}$ & & \\
\hline Mustard gas & $\mathrm{S}(\mathrm{CH} 3 \mathrm{CH} 2 \mathrm{Cl}) 2$ & $\mathrm{Cl}$ & $s$ & 121 \\
\hline m-Nitroaniline & NO2C6H4NH2 & & $\mathrm{N}$ & \\
\hline N.N-Dimethylaniline & $\mathrm{C} 6 \mathrm{H} 5 \mathrm{~N}(\mathrm{CH} 3)_{2}$ & & $\mathrm{~N}$ & \\
\hline Naphthalene & $\mathrm{ClOH} 8$ & & & 98 \\
\hline Naphthylamine (alpha-, beta-) & $\mathrm{C} 10 \mathrm{H} 7 \mathrm{NH} 2$ & & $\mathrm{~N}$ & 148 \\
\hline Nitrilotriacetic acid & $\mathrm{N}(\mathrm{CH} 2 \mathrm{COOH}) 3$ & & $\mathrm{~N}$ & \\
\hline Nitrobenzene & $\mathrm{C} 6 \mathrm{H} 5 \mathrm{NO} 2$ & & $\mathrm{~N}$ & $272,307,336.357,373$ \\
\hline $\begin{array}{l}\text { Nitrofen [Benzene, 2,4-dichloro-1- } \\
\text { (4-nitrophenoxy)-] }\end{array}$ & $\mathrm{C} 12 \mathrm{H} 7 \mathrm{Cl} 2 \mathrm{NO} 3$ & $\mathrm{Cl}$ & $\mathrm{N}$ & \\
\hline $\begin{array}{l}\text { Nitrogen mustard (2-Chloro-N-(2- } \\
\text { chloroethyl)-N-methylethanamine) }\end{array}$ & $(\mathrm{ClCH} 2 \mathrm{CH} 2) 2 \mathrm{NCH} 3$ & $\mathrm{Cl}$ & $\mathrm{N}$ & \\
\hline Nitroglycerin & - $2 \mathrm{H} 2 \mathrm{NO} 3 \mathrm{CHNO} 3 \mathrm{CH} 2 \mathrm{NO} 3$ & & $\mathrm{~N}$ & $317, \mathrm{~g} 318 . \mathrm{g} 450$ \\
\hline Nitrophenol & $\mathrm{NO} 2 \mathrm{C} 6 \mathrm{H} 4 \mathrm{OH}$ & & $\mathrm{N}$ & 19,307 \\
\hline n-Butyl alcohol & $\mathrm{CH} 3(\mathrm{CH} 2) 2 \mathrm{CH} 2 \mathrm{OH}$ & & & \\
\hline n-Dioctyl phthalate & (C8H17OOC) $2 \mathrm{C} 6 \mathrm{H} 4$ & & & \\
\hline N-Nitrosodiethylamine & C4H ION20 & & $\mathrm{N}$ & \\
\hline $\mathrm{N}$-Nitrosodimethylamine & $(\mathrm{CH} 3) 2 \mathrm{~N} 2 \mathrm{O}$ & & $\mathrm{N}$ & \\
\hline N-Nitrosodiphenylamine & (C6H5)2NNO & & $\mathrm{N}$ & \\
\hline N-Nitrosodi-n-butylamine & ONN(n-C4H9)2 & & $\mathrm{N}$ & \\
\hline N-Nitrosodi-n-propylamine & $\mathrm{ONN}(\mathrm{n}-\mathrm{C} 3 \mathrm{H} 7) 2$ & & $\mathrm{~N}$ & \\
\hline N-Nitrosomethylvinylamine & $\mathrm{ONN}(\mathrm{CH} 3)(\mathrm{C} 2 \mathrm{H} 3)$ & & $\mathrm{N}$ & \\
\hline N-Niuosomorpholine & ONNC4H8O & & $\mathrm{N}$ & \\
\hline N-Nitrosonomicotine & & & $\mathrm{N}$ & \\
\hline N-Niuosopiperidine & C5H1ONHNO & & $\mathrm{N}$ & \\
\hline N-Niuoso-N-ethylurea & $\mathrm{C}(\mathrm{O})(\mathrm{NH} 2) \mathrm{N}(\mathrm{NO}) \mathrm{C} 2 \mathrm{H} 5$ & & $\mathrm{~N}$ & \\
\hline N-Nitroso-N-methylurea & $\mathrm{C}(\mathrm{O})(\mathrm{NH} 2) \mathrm{N}(\mathrm{NO})(\mathrm{CH} 3)$ & & $\mathrm{N}$ & \\
\hline n-Pentane & $\mathrm{CH} 3(\mathrm{CH} 2) 3 \mathrm{CH} 3$ & & & \\
\hline Octachloronaphthalene & $\mathrm{ClOCl} 8$ & $\mathrm{Cl}$ & & \\
\hline Octane & $\mathrm{CH} 3(\mathrm{CH} 2) 6 \mathrm{CH} 3$ & & & \\
\hline Oxirane & $\mathrm{H} 2 \mathrm{COCH} 2$ & & & \\
\hline o-Anisidine & $\mathrm{CH} 3 \mathrm{OC} 6 \mathrm{H} 4 \mathrm{NH} 2$ & & $\mathrm{~N}$ & \\
\hline o-Anisidine hydrochloride & $\mathrm{CH} 3 \mathrm{OC} 6 \mathrm{H} 4 \mathrm{NH} 2 . \mathrm{HCl}$ & $\mathrm{Cl}$ & $\mathrm{N}$ & \\
\hline o-Nitroaniline & NO2C6H4NH2 & & $\mathrm{N}$ & \\
\hline o-Toluidine & $\mathrm{CH} 3 \mathrm{C} 6 \mathrm{H} 4 \mathrm{NH} 2$ & & $\mathrm{~N}$ & \\
\hline o-Toluidine hydrochloride & $\mathrm{CH} 3 \mathrm{C} 6 \mathrm{H} 4 \mathrm{NH} 2 . \mathrm{HCl}$ & $\mathrm{Cl}$ & & \\
\hline Parathion (DNTP) & $(\mathrm{C} 2 \mathrm{H} 5 \mathrm{O}) 2 \mathrm{P}(\mathrm{S}) \mathrm{OC} 6 \mathrm{H} 4 \mathrm{NO} 2$ & & P,S & \\
\hline PCBs (Aroclor $1260,1254,1248$, and 1242) & C 12ClxH IO-x & $\mathrm{Cl}$ & & $88,359,529536$ \\
\hline Pentachlorobenzene & $\mathrm{C} 6 \mathrm{Cl} 5 \mathrm{H}$ & $\mathrm{Cl}$ & & \\
\hline Pentachlorophenol & $\mathrm{C} 6 \mathrm{Cl} 5 \mathrm{OH}$ & $\mathrm{Cl}$ & & $5,52,130,277,305,306,423$ \\
\hline
\end{tabular}




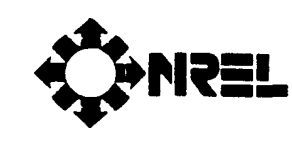

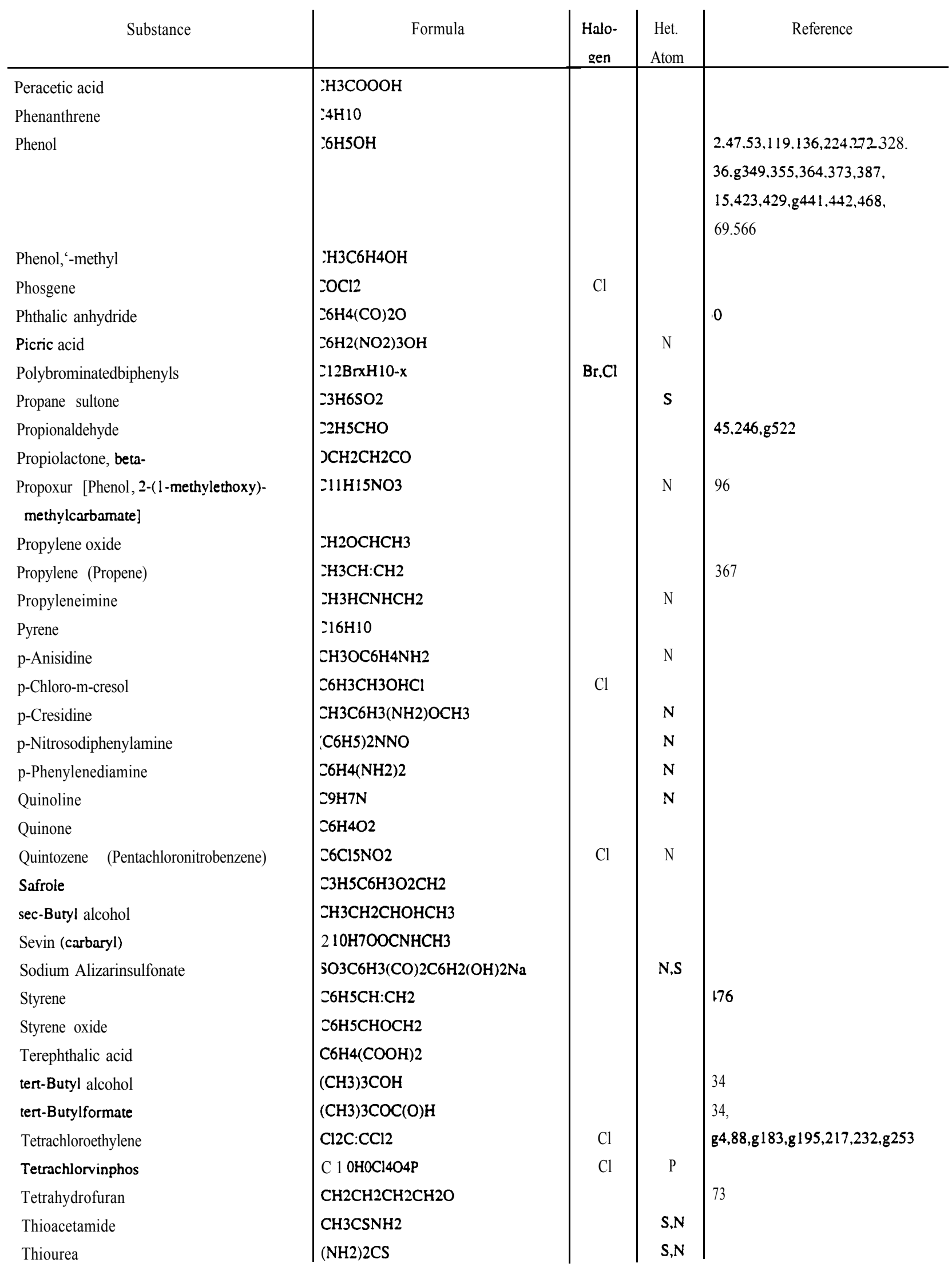




\begin{tabular}{|c|c|c|c|c|}
\hline Substance & Formula & $\begin{array}{l}\begin{array}{r}\text { Halo- } \\
\mathrm{g}\end{array} \mathrm{e} \quad \mathrm{n} \\
\end{array}$ & $\begin{array}{l}\text { Het. } \\
\text { Atom }\end{array}$ & Reference \\
\hline Tol uene & $\mathrm{C} 6 \mathrm{H} 5 \mathrm{CH} 3$ & & & $\begin{array}{l}\text { g16.g43.66,110.137.138.g169, } \\
\text { g195.269,270,g329.330.336, } \\
g 403,448.470,530\end{array}$ \\
\hline Toluene diisocyanate & $\mathrm{CH} 3 \mathrm{C} 6 \mathrm{H} 3(\mathrm{NCO}) 2$ & & $\mathrm{~N}$ & \\
\hline Total xylenes & $\mathrm{C} 6 \mathrm{H} 4(\mathrm{CH} 3) 2$ & & & \\
\hline Toxaphene & $\mathrm{ClOH} 10 \mathrm{Cl} 8$ & $\mathrm{Cl}$ & & \\
\hline Triaziquone & $\mathrm{Cl} 2 \mathrm{H} 13 \mathrm{~N} 3 \mathrm{O} 2$ & & $\mathrm{~N}$ & \\
\hline Trichlorfon & $(\mathrm{CH} 3 \mathrm{O}) 2 \mathrm{P}(\mathrm{O}) \mathrm{CH}(\mathrm{OH}) \mathrm{CCl} 3$ & $\mathrm{Cl}$ & $\mathrm{P}$ & \\
\hline Trichloroethylene & $\mathrm{CHCl}: \mathrm{CCl} 2$ & $\mathrm{Cl}$ & & $\begin{array}{l}\mathrm{g} 4, \mathrm{~g} 11,40, \mathrm{~g} 43,167, \mathrm{~g} 174 . \mathrm{g} 183, \\
\mathrm{~g} 195, \mathrm{~g} 196, \mathrm{~g} 198,217.232 .239, \\
240, \mathrm{~g} 252, \mathrm{~g} 253,269.270 .288 \\
327,346, \mathrm{~g} 384, \mathrm{~g} 445,448 . \mathrm{g} 494, \\
\mathrm{~g} 485, \mathrm{~g} 496,523,532\end{array}$ \\
\hline Triethylamine & $\mathrm{N}(\mathrm{C} 2 \mathrm{H} 5) 3$ & & $\mathrm{~N}$ & \\
\hline Trifluralin & $\mathrm{F} 3 \mathrm{C}(\mathrm{NO} 2) 2 \mathrm{C} 6 \mathrm{H} 2 \mathrm{~N}(\mathrm{C} 3 \mathrm{H} 7) 2$ & $\mathrm{~F}$ & $\mathrm{~N}$ & \\
\hline Tnnitrophenylmethylnitramine & (NO2)3C6H2N(NO2)CH3 & & $\mathrm{N}$ & \\
\hline Tris(2.3-dibromopropyl) phosphate & (CH2BrCHBrCH2O)3PO & $\mathrm{Br}$ & $P$ & \\
\hline Urethane (ethyl carbamate) & $\mathrm{CO}(\mathrm{NH} 2) \mathrm{OC} 2 \mathrm{H} 5$ & & $\mathrm{~N}$ & \\
\hline Vinyl acetate & $\mathrm{CH} 3 \mathrm{COOCH}: \mathrm{CH} 2$ & & & \\
\hline Vinyl bromide & $\mathrm{CH} 2 \mathrm{CHBr}$ & $\mathrm{Br}$ & & \\
\hline Vinyl chloride & $\mathrm{CH} 2: \mathrm{CHCl}$ & $\mathrm{Cl}$ & & $g 43$ \\
\hline Vinylidene chloride & $\mathrm{CH} 2: \mathrm{CCl} 2$ & $\mathrm{Cl}$ & & \\
\hline Xylene (mixed isomers) & $\mathrm{C} 6 \mathrm{H} 4(\mathrm{CH} 3) 2$ & & & $\begin{array}{l}110,137.138,269,313,330, g 403 . \\
470,530\end{array}$ \\
\hline Zineb & $\mathrm{Zn}(\mathrm{CS} 2 \mathrm{NHCH} 2) 2$ & & S,N & \\
\hline
\end{tabular}


Table 2. Other Organic Compounds Treated by a Photocatalytic Process

\begin{tabular}{|c|c|c|c|c|}
\hline Substance & Formula & $\begin{array}{l}\text { Halo- } \\
\text { gen }\end{array}$ & $\begin{array}{c}\text { Het. } \\
\text { Atom }\end{array}$ & Reference \\
\hline 1,1.1,2-Tetrachloroethane & $\mathrm{O} 13 \mathrm{CCH} 2 \mathrm{Cl}$ & $\mathrm{Cl}$ & & 275 \\
\hline 1,1.1-Trifluoro-2.2.2-trichioroethane & F3CCCL3 & $\mathrm{F}, \mathrm{Cl}$ & & \\
\hline 1.1,1-Trifluorobromochloroethane & $22 \mathrm{HF} 3 \mathrm{ClBr}$ & $\mathrm{F}$ & & \\
\hline 1. I-Difluoro-I ,2,2-trichloroethane & $\mathrm{IF} 2 \mathrm{CCHCl} 2$ & $\mathrm{~F} . \mathrm{Cl}$ & & \\
\hline 1, I-Difluoro-1,2-dichloroethane & $=\mathrm{ClCCClH} 2$ & $\mathrm{~F}, \mathrm{Cl}$ & & \\
\hline 1,1-Difluoroethylene & IH2CF2 & $\mathrm{F}$ & & \\
\hline 1,2-Dimethoxybenzene & $\mathrm{CH} 3 \mathrm{O}) 2 \mathrm{C} 6 \mathrm{H} 4$ & & & :72 \\
\hline 1,2-Bis(2-chloroethoxy)ethane & $\mathrm{ClC} 2 \mathrm{H} 4) 2 \mathrm{C} 2 \mathrm{H} 4$ & $\mathrm{Cl}$ & & 506 \\
\hline I,2,4,5-Tetramethylbenzene & (CH3)4C6H2 & & & 113 \\
\hline 1.3-Diphenylisobenzofuran & (C6H5)2C6H2OC6H4 & & & \\
\hline I-Benzylnicotinamide & $(\mathrm{C} 6 \mathrm{H} 5) \mathrm{CH} 2(\mathrm{C} 5 \mathrm{H} 3 \mathrm{~N}) \mathrm{C}(\mathrm{O}) \mathrm{NH} 2$ & & $\mathrm{~N}$ & 133 \\
\hline 1-Bromodecane & $\mathrm{BrClOH} 21$ & $\mathrm{Br}$ & & \\
\hline 1-Bromododecane & $3 \mathrm{rC} 12 \mathrm{H} 25$ & $\mathrm{Br}$ & & \\
\hline 1-Butanol & $\mathrm{ZH} 3(\mathrm{CH} 2) 3 \mathrm{OH}$ & & & \\
\hline 1 -Dodecanol & $\mathrm{ZH} 3(\mathrm{CH} 2) 11 \mathrm{OH}$ & & & \\
\hline 1-Hexene & $26 \mathrm{H} 12$ & & & \\
\hline 1-(Methoxyphenyl)-2-propanol & : $\mathrm{CH} 3 \mathrm{OC} 6 \mathrm{H} 4)(\mathrm{CH} 3) \mathrm{CHOH}$ & & & 25 \\
\hline 1 -Propanol & $\mathrm{n}-\mathrm{C} 3 \mathrm{H} 7 \mathrm{OH}$ & & & $245,246,247$ \\
\hline 2.3-, 2,4- or 3,4-Difluorophenol & $\mathrm{F} 2 \mathrm{C} 6 \mathrm{H} 3 \mathrm{OH}$ & $\mathrm{F}$ & & 429 \\
\hline Tris-(2.4-dichlorophenoxy)ethylphosphite & $\mathrm{C} 2 \mathrm{H} 5 \mathrm{P}[\mathrm{OC} 6 \mathrm{H} 3 \mathrm{Cl} 2]_{3}$ & $\mathrm{Cl}$ & $\mathrm{P}$ & \\
\hline 2,6-Dichlorophenol & $\mathrm{C} 6 \mathrm{H} 3 \mathrm{Cl} 2 \mathrm{OH}$ & $\mathrm{Cl}$ & & \\
\hline 2,7-Dichlorodibenzo-p-dioxin & $\mathrm{Cl} 2 \mathrm{C} 12 \mathrm{H} 6 \mathrm{O} 2$ & $\mathrm{Cl}$ & & \\
\hline 2-, 3-, 4-Fluorophenol & FC6H4OH & $\mathrm{F}$ & & 129 \\
\hline 2-Chlorodibenzo-p-dioxin & $2 \mathrm{IC} 12 \mathrm{H} 7 \mathrm{O} 2$ & $\mathrm{Cl}$ & & \\
\hline 2-Chloroethylmethylsulfide & $\mathrm{OICH} 2 \mathrm{CH} 2 \mathrm{SCH} 3$ & $\mathrm{Cl}$ & S & \\
\hline 2.3-Benzofuran & $\mathrm{C} 8 \mathrm{H} 6 \mathrm{O}$ & & & \\
\hline 2,3- and 2,5-Dichlorophenol & $\mathrm{Cl} 2 \mathrm{C} 6 \mathrm{H} 3 \mathrm{OH}$ & $\mathrm{Cl}$ & & \\
\hline 2,5-Dinitrophenol & (NO2)2C6H3OH & $\mathrm{CI}$ & $\mathrm{N}$ & \\
\hline 2,6-Dichloroindophenol & $\mathrm{C} 8 \mathrm{H} 2 \mathrm{~N}(\mathrm{OH}) \mathrm{Cl} 2$ & & & \\
\hline 2.5-Furandimethanol & $\mathrm{C} 4 \mathrm{H} 2 \mathrm{O}(\mathrm{CH} 2 \mathrm{OH}) 2$ & & & 388 \\
\hline 2-Chloroaniline & $\mathrm{ClC} 6 \mathrm{H} 4 \mathrm{NH} 2$ & $\mathrm{Cl}$ & $\mathrm{N}$ & 525 \\
\hline 2-Coumaranone & $\mathrm{C} 8 \mathrm{H} 6 \mathrm{O} 2$ & & & \\
\hline 2-Furoic Acid & (CH2)3CHOCO2H & & & \\
\hline 2-Hydroxypyridine & HOC5H4N & & $\mathrm{N}$ & 271 \\
\hline 2-Hydroxytetrahydropyran & HOC5H9O & & & 59 \\
\hline 2-Naphthol & $\mathrm{ClOH} 7 \mathrm{OH}$ & & & \\
\hline 3,3,3-Trifluoropropene & $\mathrm{CH} 2 \mathrm{CHCF} 3$ & & & \\
\hline 3,3'-Dichlorobiphenyl & $(\mathrm{ClC} 6 \mathrm{H} 4) 2$ & $\mathrm{Cl}$ & & \\
\hline
\end{tabular}




\begin{tabular}{|c|c|c|c|c|}
\hline Substance & Formula & $\begin{array}{l}\text { Halo- } \\
\text { gen }\end{array}$ & $\begin{array}{l}\text { Het. } \\
\text { Atom }\end{array}$ & Reference \\
\hline 3.4-Chlorophenol & $3,4-\mathrm{Cl} 2 \mathrm{C} 6 \mathrm{H} 3 \mathrm{OH}$ & $\mathrm{Cl}$ & & \\
\hline 3-Chlorophenol & $\mathrm{m}-\mathrm{ClC} 6 \mathrm{H} 4 \mathrm{OH}$ & $\mathrm{Cl}$ & & $175.296,497$ \\
\hline 3-Chlorosalicylic acid & $\mathrm{C} 7 \mathrm{H} 5 \mathrm{ClO} 3$ & $\mathrm{Cl}$ & & \\
\hline 4-Bromophenol & BrC6H4OH & $\mathrm{Br}$ & & 328 \\
\hline 4-t-Butyltoluene & $\mathrm{p}-(\mathrm{t}-\mathrm{C} 4 \mathrm{H} 9) \mathrm{C} 6 \mathrm{H} 4 \mathrm{CH} 3$ & & & \\
\hline 4-Chloro-3-nitro-benzotrifluoride & $\mathrm{C} 6 \mathrm{HCl}(\mathrm{NO} 2) \mathrm{F} 3$ & $\mathrm{~F}, \mathrm{Cl}$ & N & \\
\hline 4-Chlorophenol & $\mathrm{ClC} 6 \mathrm{H} 4 \mathrm{OH}$ & $\mathrm{Cl}$ & & $\begin{array}{l}108,140,141,142,172.173,175.277, \\
279,296,298,303,328,394, \\
443, g 444,481,484,485,497, \\
512,513,514,521\end{array}$ \\
\hline 4-Chlorophenylisocyanate & $\mathrm{CIC} 6 \mathrm{H} 4 \mathrm{NCO}$ & $\mathrm{Cl}$ & N & 375 \\
\hline 4-Fluorophenol & FC6 $440 H$ & $\mathrm{~F}$ & & 328 \\
\hline 4-Hydroxyacetophenone & $\mathrm{HOC} 6 \mathrm{H} 4 \mathrm{C}(\mathrm{O}) \mathrm{CH} 3$ & & & 328 \\
\hline 4-Hydroxybenzyl Alcohol & $\mathrm{p}-\mathrm{HO}(\mathrm{C} 6 \mathrm{H} 4) \mathrm{CH} 2 \mathrm{OH}$ & & & 386 \\
\hline 4-Iodophenol & IC6H4OH & I & & 328 \\
\hline 4-Methoxyphenol & $\mathrm{CH} 3 \mathrm{C} 6 \mathrm{H} 4 \mathrm{OH}$ & & & 328 \\
\hline 4-Nitroaniline & $\mathrm{NO} 2 \mathrm{C} 6 \mathrm{H} 4 \mathrm{NH} 2$ & & N & 442 \\
\hline 4-Nitrocatechol & $(\mathrm{NO} 2) \mathrm{C} 6 \mathrm{H} 3(\mathrm{OH}) 2$ & & $\mathbf{N}$ & \\
\hline 4-Niuophenylethylphosphinate & $(\mathrm{NO} 2) \mathrm{C} 6 \mathrm{H} 4(\mathrm{C} 2 \mathrm{H} 5) \mathrm{PO} 2$ & & $\mathrm{~N}, \mathrm{P}$ & \\
\hline 4-Nitrophenylisopropylphosphinate & & & N.P & \\
\hline 4-nitrophenyldiethylphosphate & & & N.P & \\
\hline 4-Thiophenyl- 1 -butanol & $\mathrm{C} 6 \mathrm{H} 5 \mathrm{~S}(\mathrm{CH} 2) 4 \mathrm{OH}$ & & $\mathbf{S}$ & \\
\hline 4-Trifluoromethylphenol & $\mathrm{CF} 3 \mathrm{C} 6 \mathrm{H} 4 \mathrm{OH}$ & $\mathrm{F}$ & & 328 \\
\hline 5-Fluorouracil & $\mathrm{FC} 4 \mathrm{H}(\mathrm{NH}) 2(\mathrm{O}) 2$ & $\mathrm{~F}$ & & 12 \\
\hline 5-Hydroxypentanoic acid & $\mathrm{HO}(\mathrm{CH} 2) 4 \mathrm{CO} 2 \mathrm{H}$ & & & \\
\hline 12-phenyldodecanesulfonate, Sodium Salt & $\mathrm{C} 6 \mathrm{H} 5(\mathrm{CH} 2) 12 \mathrm{SO} 3 \mathrm{H}$ & & & \\
\hline Acenapthane & $\mathrm{ClOH} 16(\mathrm{CH} 2) 2$ & & & 98,188 \\
\hline Acetic Acid & $\mathrm{CH} 3 \mathrm{CO} 2 \mathrm{H}$ & & & $\begin{array}{l}52,203,210,212,275,354, g 416, \\
g 417\end{array}$ \\
\hline Acetophenone & $\mathrm{CH} 3 \mathrm{COC} 6 \mathrm{H} 5$ & & & \\
\hline Acetylene & $\mathrm{C} 2 \mathrm{H} 2$ & & & 236,254 \\
\hline Acid orange 7 & $\mathrm{Na} .03 \mathrm{SC} 6 \mathrm{H} 4 \mathrm{~N} 2 \mathrm{Cl} 10 \mathrm{H} 6 \mathrm{OH}$ & & N.S & $480,482,483$ \\
\hline Adipic acid & $\mathrm{CSH} 11 \mathrm{CO} 2 \mathrm{H}$ & & & 147 \\
\hline Aldicarb & $\mathrm{CH} 3 \mathrm{SC}(\mathrm{CH} 3) 2 \mathrm{CHN}(\mathrm{O}) \mathrm{C}(\mathrm{O}) \mathrm{NHCH} 3$ & & $\mathrm{~N}, \mathrm{~S}$ & 5 \\
\hline p-alkylphenol (various) & $\mathrm{R}(\mathrm{C} 6 \mathrm{H} 4) \mathrm{OH}$ & & & \\
\hline Allyl alcohol & $\mathrm{C} 3 \mathrm{H} 5 \mathrm{OH}$ & & & \\
\hline Alochlor & & & & \\
\hline p-Aminophenol & $\mathrm{NH} 2(\mathrm{C} 6 \mathrm{H} 4) \mathrm{OH}$ & & $\mathrm{N}$ & $74,79,101$ \\
\hline Anthraquinone-2-sulfonic acid & $\mathrm{HO} 3 \mathrm{SC} 14 \mathrm{H} 7 \mathrm{O} 2$ & & 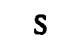 & 227 \\
\hline AOX or Haloform Precursors & & & & $248,249,434,435$ \\
\hline Asulam & & & & \\
\hline Azobenzenes (various) & $\mathrm{XC6H} 4 \mathrm{NNC} 6 \mathrm{H} 4 \mathrm{X}$ & & $\mathbf{N}$ & 103 \\
\hline Azobisformamidoaceuc acid & & & $\mathbf{N}$ & 490 \\
\hline
\end{tabular}




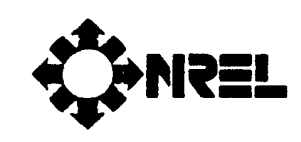

\begin{tabular}{|c|c|c|c|c|}
\hline Substance & Formula & $\begin{array}{c}\text { Halo- } \\
\text { g } \\
\end{array}$ & $\begin{array}{l}\text { Het. } \\
\text { Atom }\end{array}$ & Reference \\
\hline Bacteria/Algae/Virus & & & & $\begin{array}{l}\text { 15,55,123,124.125, 126,g139,189, } \\
216.283,284.333,365,391,401, \\
437,503,505,507\end{array}$ \\
\hline Benzaldehyde & $\mathrm{C} 6 \mathrm{H} 5 \mathrm{C}(\mathrm{O}) \mathrm{H}$ & & & 476 \\
\hline Benzophenone & (C6H5)2CO & & & 323 \\
\hline Benzoquinone & $26 \mathrm{H} 4 \mathrm{O} 2$ & & & $272,385.387$ \\
\hline Benzyldodecyldimethylarnonium chloride & (C6H5CH2)(C12H25)(CH3)2N,Cl & $\mathrm{Cl}$ & $\mathrm{N}$ & 161,533 \\
\hline Benzyltetradecyldimethylamonium chloride & . $66 \mathrm{H} 5 \mathrm{CH} 2)(\mathrm{Cl} 4 \mathrm{H} 27)(\mathrm{CH} 3) 2 \mathrm{~N}, \mathrm{Cl}$ & $\mathrm{Cl}$ & $\mathrm{N}$ & 158,160 \\
\hline Biomass & & & & 187 \\
\hline Biphthalate & (C6H4) $(\mathrm{CO} 2 \mathrm{H}) \mathrm{CO} 2)(-1)$ & & & \\
\hline Bromobentene & $\mathrm{BrC6H5}$ & $\mathrm{Br}$ & & 336 \\
\hline Butane & $24 \mathrm{H} 10$ & & & \\
\hline But-2-ene. trans & $\mathrm{Z} 33 \mathrm{CHCHCH} 3$ & & & g181 \\
\hline Butyl alcohol & , $-\mathrm{C} 4 \mathrm{H} 7 \mathrm{OH}$ & & & 53 \\
\hline Butylamine & 1-C4H7NH2 & & $\mathrm{N}$ & 75 \\
\hline t-Butylazine & & & $\mathrm{N}$ & 296,393 \\
\hline Butadiene & 2 $\mathrm{H} 2 \mathrm{CHCHCH} 2$ & & & 3329 \\
\hline Butyric acid & $23 \mathrm{H} 7 \mathrm{CO} 2 \mathrm{H}$ & & & 135,354 \\
\hline Carbetamide & & & $\mathrm{N}$ & 54 \\
\hline Carbon dioxide (reduction) & 202 & & & $166,186,19 \mathrm{I}, 282.334,438,460$ \\
\hline Carbon monoxide & 30 & & & $3264, g 405, g 452$ \\
\hline Carbon tetrabromide & $\mathrm{zBr} 4$ & $\mathrm{Br}$ & & \\
\hline Catechol & $26 \mathrm{H} 4(\mathrm{OH}) 2$ & & & 521 \\
\hline Cetyldimethylbenzylamonium chloride & $\mathrm{ZH} 3(\mathrm{CH} 2) 15(\mathrm{CH} 3) 2(\mathrm{C} 6 \mathrm{H} 5 \mathrm{CH} 2) \mathrm{N}, \mathrm{Cl}$ & & $\mathrm{N}$ & 200,370 \\
\hline Cetylpyridinium chloride & $\mathrm{V}-[\mathrm{CH} 3(\mathrm{CH} 2)[5](\mathrm{C} 5 \mathrm{H} 5 \mathrm{~N}), \mathrm{Cl}$ & & $\mathrm{N}$ & 370 \\
\hline Chloroacetaldehyde & $\mathrm{IH} 2 \mathrm{ClC}(\mathrm{O}) \mathrm{H}$ & $\mathrm{Cl}$ & & 275 \\
\hline Chlorobenzoic acids, o-, m-, or p- & $\mathrm{Il}(\mathrm{C} 6 \mathrm{H} 4) \mathrm{CO} 2 \mathrm{H}$ & $\mathrm{Cl}$ & & 33 \\
\hline Chlorofluorocarbons, various & & $\mathrm{F}, \mathrm{C}$ & & \\
\hline Chloral hydrate & $\mathrm{Zl} 3 \mathrm{CO}(\mathrm{OH}) 2$ & $\mathrm{cl}$ & & 275.534 \\
\hline Chloranil, o- and p- & $26 \mathrm{Cl} 14 \mathrm{O} 2$ & $\mathrm{Cl}$ & & \\
\hline Chloroethylammonium chloride & $2 \mathrm{IH} 3 \mathrm{~N} . \mathrm{Cl}$ & $\mathrm{Cl}$ & $\mathrm{N}$ & \\
\hline Chlorpyrifos & & $\mathrm{Cl}$ & $S, N$ & 127 \\
\hline Ciba Orange RI & & & & 528 \\
\hline Coal or Carbon & & & & 567 \\
\hline Color and/or COD (in wastewater) & & & & $\begin{array}{l}13,100,103,251,332,434,435 \\
159.465\end{array}$ \\
\hline Congo Red & $232 \mathrm{H} 22 \mathrm{O} 6 \mathrm{~N} 6 \mathrm{~S} 2 \mathrm{Na2}$ & & & \\
\hline Cresol violet & $216 \mathrm{H} 8 \mathrm{NO}(\mathrm{NH} 2), \mathrm{Cl}$ & & $\mathrm{N}$ & \\
\hline Creosote phenolics & & & & 164 \\
\hline Cyanuric acid & $23 \mathrm{~N} 3(\mathrm{OH}) 3$ & & $\mathrm{~N}$ & \\
\hline Cyclododecanol & $=12 \mathrm{H} 23 \mathrm{OH}$ & & & 120 \\
\hline Cyclohexane & $26 \mathrm{H} 12$ & & & $\mathrm{~g} 368, \mathrm{~g} 508$ \\
\hline Cyclohexanedicarboxylic Acids & $26 \mathrm{H} 10(\mathrm{CO} 2 \mathrm{H}) 2$ & & & \\
\hline
\end{tabular}




\begin{tabular}{|c|c|c|c|c|}
\hline Substance & Formula & \multirow[t]{6}{*}{$\begin{array}{l}\text { Halo- } \\
\text {-g e n }\end{array}$} & $\begin{array}{c}\text { Her. } \\
\text { Atom } \\
\end{array}$ & Reference \\
\hline Cyclohexanol & $=6 \mathrm{H} 11 \mathrm{OH}$ & & & 120.442 \\
\hline Cyclohexene & $26 \mathrm{H} 10$ & & & \\
\hline Cyclohexene oxide & $=6 \mathrm{H} 10 \mathrm{O}$ & & & \\
\hline Cyclophosphamide & JPONHC3H6[N(C2H4Cl)2] & & $\therefore N$ & \\
\hline Cynnamyl alcohol & $26 \mathrm{H} 4 \mathrm{C} 2 \mathrm{H} 2 \mathrm{OH}$ & & & \\
\hline DDT & $\mathrm{ClC} 6 \mathrm{H} 4) 2 \mathrm{CHCCl} 3$ & $\mathrm{Cl}$ & & \\
\hline Decalin & $210 \mathrm{H} 18$ & & & \\
\hline Decamethylteuasiloxane & $\mathrm{CH} 3) 10 \mathrm{Si} 4 \mathrm{O} 3$ & & $\mathrm{Si}$ & 3361 \\
\hline Decanoic acid & $29 \mathrm{H} 19 \mathrm{CO} 2 \mathrm{H}$ & & & \\
\hline Decanol & $\mathrm{HOC} 10 \mathrm{H} 21$ & & & \\
\hline Desipramine & $\mathrm{C} 6 \mathrm{H} 4) 2(\mathrm{CH} 2) 2 \mathrm{~N}(\mathrm{CH} 2) 3 \mathrm{NHCH} 3$ & & & \\
\hline Dibenzo-p-dioxines, various & & $\mathrm{Cl}$ & & 536,555 \\
\hline Dibromomethane & $\mathrm{ZH} 2 \mathrm{Br} 2$ & $\mathrm{Br}$ & & \\
\hline Dichloroacetic acid & $\mathrm{Z} 12 \mathrm{CHCO} 2 \mathrm{H}$ & $\mathrm{Cl}$ & & $40,52,167,256,275,277,392$ \\
\hline Dichloroacetyl Chloride & $=12 \mathrm{CHCOCl}$ & $\mathrm{Cl}$ & & 3239.9240 \\
\hline Dimethylamme & $\mathrm{CH} 3) 2 \mathrm{NH}$ & & $\mathrm{N}$ & 364 \\
\hline Dimethylsulfide & $\mathrm{CH} 3) 2 \mathrm{~S}$ & & $s$ & $5361, g 363$ \\
\hline Dimethyl-2.2-dichlorusinyl phosphate & $\mathrm{CH} 3) 2(\mathrm{Cl} 2 \mathrm{CCH}) \mathrm{PO} 4$ & $\mathrm{Cl}$ & $\mathrm{P}$ & 177 \\
\hline Diphenylmethane & $\mathrm{C} 6 \mathrm{H} 5) 2 \mathrm{CH} 2$ & & & \\
\hline Diphenylsulfide & $\mathrm{C} 6 \mathrm{H} 5) 2 \mathrm{~S}$ & & $s$ & \\
\hline Direct blue 1 & $(\mathrm{Na} . \mathrm{O} 3 \mathrm{~S}) \mathrm{Cl} 6 \mathrm{H} 6(\mathrm{NH} 2)(\mathrm{OH})-$ & & & \\
\hline & $(\mathrm{OCH} 3) \mathrm{N} 2] 2$ & & N.S & 180 \\
\hline Dodecane & $=12 \mathrm{H} 26$ & & & \\
\hline Dodecyl sulfate & $\mathrm{C} 12 \mathrm{H} 25) 2 \mathrm{SO} 4$ & & & \\
\hline Dodecylbenzenesulfonate & $\mathrm{Cl} 2 \mathrm{H} 25) \mathrm{C} 6 \mathrm{H} 4 \mathrm{SO} 3)(-1)$ & & $S$ & $160,161,533$ \\
\hline Dodecyldecaoxyethylenephosphates & & & $\mathrm{P}$ & 162 \\
\hline Dodecylpyridinium chloride & $\mathrm{Cl} 2 \mathrm{H} 25) \mathrm{C} 5 \mathrm{H} 5 \mathrm{NH}, \mathrm{Cl}$ & $\mathrm{I}$ & 1 & $23,156,158$ \\
\hline Doxycycline & & & & \\
\hline Eosin & & & & \\
\hline Ethambutol & & & $\mathrm{N}$ & 261 \\
\hline Ethane & $22 \mathrm{H} 6$ & & & $\zeta 487,488$ \\
\hline Ethanol & $22 \mathrm{H} 5 \mathrm{OH}$ & & & $\begin{array}{l}28,843,122,194,203, g 450, \\
454\end{array}$ \\
\hline Ethylacetate & $\mathrm{Z} \mathrm{H} 3 \mathrm{CO} 2 \mathrm{C} 2 \mathrm{H} 5$ & & & \\
\hline Ethylenediaminetetraacetic acid & $\mathrm{O} 2 \mathrm{CCH} 2) 4 \mathrm{~N} 2 \mathrm{C} 2 \mathrm{H} 4$ & & J & 259,373 \\
\hline 2-, 3-, or 4-Ethylphenol & $\mathrm{C} 2 \mathrm{H} 5) \mathrm{C} 6 \mathrm{H} 4 \mathrm{OH}$ & & & 177 \\
\hline Feniuothion & C9H12NOSPS & & ISP & \\
\hline Fluorescein & $\mathrm{C} 2 \mathrm{OH} 1205$ & & & \\
\hline Folicur & & & $\mathrm{N}$ & 491 \\
\hline Formic Acid & $\mathrm{HCO} 2 \mathrm{H}$ & & & $\begin{array}{l}2,80,117,135,223,233,275,354 \\
373,418,419,420\end{array}$ \\
\hline Fullerenes & $\mathrm{C} 60, \mathrm{C} 70$, and $\mathrm{C} 84$ & & & $207,208,408$ \\
\hline Fulvic acid & & & & 177,573 \\
\hline
\end{tabular}




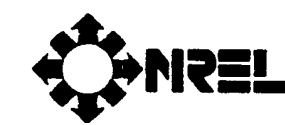

\begin{tabular}{|c|c|c|c|c|}
\hline Substance & Formula & $\begin{array}{l}\text { Halo- } \\
\text { g e n }\end{array}$ & $\begin{array}{c}\text { Het. } \\
\text { Atom }\end{array}$ & Reference \\
\hline Glycerol & $\mathrm{C} 3 \mathrm{H} 5(\mathrm{OH}) 3$ & & & \\
\hline HCFC or HFC & & $\mathrm{Cl} . \mathrm{F}$ & & g455 \\
\hline Heparin & & & & 54 \\
\hline Hexafluorobenzene & C6F6 & $\mathrm{F}$ & & 308 \\
\hline Hexafluoropropene & CF2CFCF3 & $\mathrm{F}$ & & 113 \\
\hline Hexanol & $\mathrm{C} 6 \mathrm{H} 13 \mathrm{OH}$ & & & 120 \\
\hline Humic Acids & & & & $132.170,250.332,479$ \\
\hline Hydroxybenzoic acid (various) & $\mathrm{HOC} 6 \mathrm{H} 4(\mathrm{OH}) \mathrm{CO} 2 \mathrm{H}$ & & & \\
\hline Hydroxycarboxylic acids, alpha & $\mathrm{RCH}(\mathrm{OH}) \mathrm{CO} 2 \mathrm{H}$ & & & \\
\hline Hydroxyethylcellulose & & & & \\
\hline Indole & $\mathrm{C} 8 \mathrm{H} 6 \mathrm{NH}$ & & $\mathrm{N}$ & g361 \\
\hline Isobutane & $\mathrm{C} 4 \mathrm{H} 10$ & & & 86 \\
\hline Isobutanol & $\mathrm{CH} 3 \mathrm{CH}(\mathrm{CH} 3) \mathrm{CH} 2 \mathrm{OH}$ & & & \\
\hline Isobutene & $\mathrm{C} 4 \mathrm{H} 8$ & & & \\
\hline Isobutyric Acid & $\mathrm{CH} 3 \mathrm{CH}(\mathrm{CH} 3) \mathrm{CO} 2 \mathrm{H}$ & & & \\
\hline Isoprene & $\mathrm{CH} 2 \mathrm{C}(\mathrm{CH} 3) \mathrm{CHCH} 2$ & & & g192 \\
\hline Isorsorbide dinitrate & $\mathrm{C} 6 \mathrm{O} 2 \mathrm{H} 8(\mathrm{ONO} 2) 2$ & & v & \\
\hline Iso-octane & $(\mathrm{CH} 3) 2 \mathrm{CH}(\mathrm{CH} 2) 4 \mathrm{CH} 3$ & & & g43,g253 \\
\hline L-Lysine & $\mathrm{NH} 2(\mathrm{CH} 2) 4 \mathrm{CH}(\mathrm{NH} 2) \mathrm{CO} 2 \mathrm{H}$ & & & \\
\hline Lactic acid & $\mathrm{C} 33 \mathrm{H} 6 \mathrm{O} 3$ & & & 163 \\
\hline Kraft lignin & & & & $187,337,434,435$ \\
\hline Maleic acid & $\mathrm{HO} 2 \mathrm{CCHCHCO} 2 \mathrm{H}$ & & & 135 \\
\hline Malonic acid & $\mathrm{CH} 2(\mathrm{CO} 2 \mathrm{H}) 2$ & & & $135,147,259$ \\
\hline Methane & $\mathrm{CH} 4$ & & & 3487,488 \\
\hline Methanethiol & $\mathrm{C}=\mathrm{H} 3 \mathrm{SH}$ & & & 3504 \\
\hline Methylcyclohexane & (:.H3C6H11 & & & 3368 \\
\hline Methyl orange & $\mathrm{Na}, \mathrm{O} 3 \mathrm{SC} 6 \mathrm{H} 4 \mathrm{~N} 2 \mathrm{C} 6 \mathrm{H} 4 \mathrm{~N}(\mathrm{CH} 3)_{2}$ & & N.S & $77,78,301$ \\
\hline Methyl viologen & $(\mathrm{CH} 3 \mathrm{C} 5 \mathrm{H} 4 \mathrm{~N}) 2 . \mathrm{Cl} 2$ & $\mathrm{Cl}$ & $\mathrm{N}$ & $260,30 \mathrm{I}, 501$ \\
\hline Methylene blue & $(\mathrm{CH} 3) 2 \mathrm{NC} 6 \mathrm{H} 3 \mathrm{NSC} 6 \mathrm{H} 3 \mathrm{~N}(\mathrm{CH} 3) 2, \mathrm{Cl}$ & $\mathrm{Cl}$ & N.S & $17,39,324,463,475$ \\
\hline Methylvinylketone & $\mathrm{C} \cdot \mathrm{H} 3 \mathrm{COC} 2 \mathrm{H} 3$ & & & \\
\hline Monuron & C.IC6H4NHCON(CH3)2 & $\mathrm{Cl}$ & $\mathrm{N}$ & 20,375 \\
\hline m-Phenoxytoluene & $\mathrm{n}_{2}-\mathrm{C} 6 \mathrm{H} 5 \mathrm{O}-\mathrm{C} 6 \mathrm{H} 4 \mathrm{CH} 3$ & & & \\
\hline$N, N, N^{\prime}, N^{\prime}-$ Tetraethyloxonine & & & $\mathrm{N}$ & 179 \\
\hline Naphthol & $\mathrm{ClOH} 7 \mathrm{OH}$ & & & \\
\hline Nile Blue A & $\mathrm{C} 16 \mathrm{NO}(\mathrm{NH} 2) \mathrm{N}(\mathrm{C} 2 \mathrm{H} 5) 2, \mathrm{SO} 4$ & & N,S & 179 \\
\hline Nitrocellulose & & & $\mathrm{N}$ & 12 \\
\hline p-Nitrotoluenesulfonic acid & $(\mathrm{CH} 3)(\mathrm{NO} 2) \mathrm{C} 6 \mathrm{H} 3 \mathrm{SO} 3 \mathrm{H}$ & & $\mathrm{N}, \mathrm{S}$ & 310 \\
\hline Nitrotoluene, various & $\mathrm{NO} 2 \mathrm{C} 6 \mathrm{H} 4 \mathrm{CH} 3$ & & $\mathrm{~N}$ & \\
\hline Nonylphenolethoxylate & $\mathrm{C} 9 \mathrm{H} 17 \mathrm{C} 6 \mathrm{H} 4 \mathrm{OC} 2 \mathrm{H} 5$ & & & \\
\hline Oil/Petroleum & & & & $44,152,153,200,320$ \\
\hline Oxalic acid & $\mathrm{C} 2 \mathrm{O} 4 \mathrm{H} 2$ & & & $135,147,259,273,275,354$ \\
\hline Pendimethalin & & & & 351 \\
\hline Pentafluorophenol & $\mathrm{C} 6 \mathrm{~F} 5 \mathrm{OH}$ & & & 308 \\
\hline
\end{tabular}




\begin{tabular}{|c|c|c|c|c|}
\hline Substance & Formula & $\begin{array}{l}\text { Halo- } \\
\mathrm{g} \quad \mathrm{e} \quad \mathrm{n}\end{array}$ & $\begin{array}{l}\text { Het. } \\
\text { Atom } \\
\end{array}$ & Reference \\
\hline n-Pentyl amine & n-C5H11 NH2 & & $\mathrm{N}$ & \\
\hline Permethrin & & & & 161 \\
\hline Phenosafranin & $\mathrm{C} 6 \mathrm{H} 5 \mathrm{~N} 2 \mathrm{C} 12 \mathrm{H} 4(\mathrm{NH} 2(\mathrm{CH} 3) 2$ & & $\mathrm{N}$ & 39 \\
\hline Picoline & $\mathrm{CH} 3 \mathrm{C} 5 \mathrm{H} 4 \mathrm{~N}$ & & $\mathrm{~N}$ & \\
\hline Piperidene & $\mathrm{CSHIONH}$ & & $\mathrm{N}$ & 75 \\
\hline Polyethoxylene Alkyl Ethers & $\mathrm{R} 2(\mathrm{OC} 2 \mathrm{H} 4) \mathrm{n}$ & & & 156,432 \\
\hline Polyethylene & $(\mathrm{CH} 2 \mathrm{CH} 2) \mathrm{n}$ & & & 291 \\
\hline Polypropylene & {$[(\mathrm{CH} 3) \mathrm{CHCH} 2] \mathrm{n}$} & & & 291 \\
\hline Polyvinylalcohol & $(\mathrm{C} 2 \mathrm{H} 3 \mathrm{OH}) \mathrm{n}$ & & & 213,292 \\
\hline Proline & $\mathrm{C} 4 \mathrm{H} 8 \mathrm{NCO} 2 \mathrm{H}$ & & $\mathrm{N}$ & \\
\hline Prometon & & & & \\
\hline Prometryn & & & & \\
\hline Propane & $\mathrm{C} 3 \mathrm{H} 8$ & & & g487 \\
\hline Propionic acid & $\mathrm{C} 2 \mathrm{H} 5 \mathrm{CO} 2 \mathrm{H}$ & & & \\
\hline Propylene glycol dinitrate & $\mathrm{CH} 3 \mathrm{CH}(\mathrm{NO} 3) \mathrm{CH} 2$ (NO3) & & $\mathbf{N}$ & 257 \\
\hline Propyne & $\mathrm{CH} 3 \mathrm{CCH}$ & & & \\
\hline Propyzarnide & & & $\mathbf{N}$ & 473 \\
\hline Pyridine & C5H5N & & $\mathbf{N}$ & 75.g195.27 1.g402 \\
\hline Pyrocatechol & o-C6H4(OH)2 & & & \\
\hline Pyrrole & C4H5N & & $\mathbf{N}$ & $\mathrm{g} 361$ \\
\hline Reactive Dyes & & & & 299 \\
\hline Red Dye 79 & & & $\mathrm{~N}, \mathrm{~S}$ & \\
\hline Resorcinol & $\mathrm{C} 6 \mathrm{H} 6 \mathrm{O} 2$ & & & 101 \\
\hline Rhodamine B & $\mathrm{CH} 3 \mathrm{OC}(\mathrm{O})(\mathrm{C} 6 \mathrm{H} 4) \mathrm{C} 13 \mathrm{H} 6 \mathrm{O}(\mathrm{NH} 2) 2$ & & $\mathbf{N}$ & \\
\hline Rhodamine $6 \mathrm{G}$ & $\begin{array}{l}\mathrm{C} 2 \mathrm{H} 5 \mathrm{OC}(\mathrm{O}) \mathrm{C} 6 \mathrm{H} 4 \mathrm{C} 13 \mathrm{H} 4(\mathrm{CH} 3) 2- \\
{[\mathrm{N}(\mathrm{C} 2 \mathrm{H} 5)] 2, \mathrm{Cl}}\end{array}$ & $\mathrm{Cl}$ & $\mathbf{N}$ & 297 \\
\hline Rhodamine $6 \mathrm{ZH}$ & & & & 237 \\
\hline Rose Bengal & $\mathrm{Na} 2.02 \mathrm{CC} 6 \mathrm{Cl} 4 \mathrm{C} 13 \mathrm{H} 2 \mathrm{OI} 4 \mathrm{O} 2$ & Cl.I & & 393 \\
\hline S-Dodecyl thioether carboxylates & & & $S$ & 157 \\
\hline $\begin{array}{l}\text { S-Ethyl-N,N-dipropyl thiocarbamate } \\
\text { (EPTC) }\end{array}$ & $(\mathrm{C} 2 \mathrm{H} 5) \mathrm{SC}(\mathrm{O}) \mathrm{N}(\mathrm{C} 3 \mathrm{H} 7) 2$ & & N.S & 478 \\
\hline $\begin{array}{l}\text { S-Ethyl-N.N-diisopropylthiocarbamate } \\
\text { ( Butylate) }\end{array}$ & $(\mathrm{C} 2 \mathrm{H} 5) \mathrm{SC}(\mathrm{O}) \mathrm{N}(\mathrm{i}-\mathrm{C} 3 \mathrm{H} 7) 2$ & & N.S & 478 \\
\hline $\begin{array}{l}\text { S-Ethyl-4-hexahydro-1-H-azepine-1- } \\
\text { carbothionate (molinate) }\end{array}$ & & & $\mathrm{N}, \mathrm{S}$ & 478 \\
\hline $\begin{array}{l}\text { S-Propyl-N-cyclohexyl thiocarbamate } \\
\text { (cycloate) }\end{array}$ & $(\mathrm{C} 3 \mathrm{H} 7) \mathrm{SC}(\mathrm{O}) \mathrm{NH}(\mathrm{C} 6 \mathrm{H} 11)$ & & $\mathrm{N}, \mathrm{S}$ & 478 \\
\hline $\begin{array}{l}\text { S-Propyl-N,N-dipropyl thiocarbamate } \\
\text { (vemolate) }\end{array}$ & $(\mathrm{C} 3 \mathrm{H} 7) \mathrm{SC}(\mathrm{O})(\mathrm{NC} 3 \mathrm{H} 7) 2$ & & N.S & 478 \\
\hline Salicylic acid & $\mathrm{C} 7 \mathrm{H} 6 \mathrm{O} 3$ & & & $\begin{array}{l}7,92,95,97,99,221,302,373,376 \\
448,454,466.521,524\end{array}$ \\
\hline Simazine & $(\mathrm{C} 2 \mathrm{H} 5) \mathrm{Cl}(\mathrm{NHC} 2 \mathrm{H} 5) \mathrm{C} 3 \mathrm{~N} 3$ & & & \\
\hline Sodium chloroacetate & $\mathrm{CH} 3 \mathrm{CO} 2 \mathrm{Na}$ & $\mathrm{Cl}$ & & \\
\hline
\end{tabular}




\begin{tabular}{|c|c|c|c|c|}
\hline Substance & Formula & \multirow{6}{*}{$\begin{array}{l}\text { Halo- } \\
\text { g e n }\end{array}$} & & Reference \\
\hline Sodium dodecylbenzene sulfonate & $=12 \mathrm{H} 25 \mathrm{C} 6 \mathrm{H} 4 \mathrm{SO} 3 \mathrm{Na}$ & & $S$ & \\
\hline$S$ tilbene & $26 \mathrm{H} 5 \mathrm{CHCHC} 6 \mathrm{H} 5$ & & & \\
\hline Succinic acid & $1 \mathrm{O} 2 \mathrm{CCH} 2 \mathrm{CH} 2 \mathrm{CO} 2 \mathrm{H}$ & & & 35.147 .163 \\
\hline Sucrose & $212 \mathrm{H} 22 \mathrm{O} 11$ & & & \\
\hline Sulfones & $2 \mathrm{~S}(\mathrm{O}) 2 \mathrm{R}^{\prime}$ & & $\mathbf{S}$ & 139 \\
\hline Tetrachlorvinphos & $\mathrm{ZHClCH}(2,4,5-\mathrm{Cl} 3 \mathrm{C} 6 \mathrm{H} 2)(\mathrm{CH} 3) \mathrm{PO} 4$ & $\mathrm{Cl}$ & $\mathrm{P}$ & $\because 18$ \\
\hline Tetrafluoroethylene & $22 \mathrm{~F} 4$ & $\mathrm{~F}$ & & \\
\hline Tetralin & $210 \mathrm{H} 12$ & & & \\
\hline Tetramethylenediamine & $\mathrm{NH} 2(\mathrm{CH} 2) 4 \mathrm{NH} 2$ & & $\mathrm{~N}$ & \\
\hline Tetrabutylammonium phosphate & {$[(\mathrm{n}-\mathrm{C} 4 \mathrm{H} 9) 4 \mathrm{~N}] 4, \mathrm{PO} 4$} & & $\mathrm{P}$ & \\
\hline Theophylline & $\mathrm{C} 7 \mathrm{H} 8 \mathrm{~N} 4 \mathrm{O} 2 . \mathrm{H} 2 \mathrm{O}$ & & $N$ & \\
\hline Thioethers & RSR' & & $\mathbf{S}$ & 121 \\
\hline Thiobencarb & & & & 322.336 \\
\hline Thymine & $25 \mathrm{H} 6 \mathrm{~N} 2 \mathrm{O} 2$ & & $\mathrm{~N}$ & \\
\hline Thionine & $312 \mathrm{NS}(\mathrm{NH} 2), \mathrm{O} 2 \mathrm{CCH} 3$ & & $\mathrm{~S}, \mathrm{~N}$ & 19 \\
\hline p-Toluenesulfonic acid & $\mathrm{ZH} 3(\mathrm{C} 6 \mathrm{H} 4) \mathrm{SO} 3 \mathrm{H}$ & & $\mathrm{S}$ & 50.62 \\
\hline $\mathrm{s}$-Triazines & & & $\mathrm{N}$ & 274 \\
\hline Trichloroacetic acid & $\mathrm{Cl} 3 \mathrm{CCO} 2 \mathrm{H}$ & $\mathrm{Cl}$ & & 10.275 \\
\hline Trietazine & & & $\sqrt{1}$ & \\
\hline Triethanolamine & $\mathrm{N}(\mathrm{CH} 2 \mathrm{CH} 2 \mathrm{OH}) 3$ & & J & 433 \\
\hline Trifluoroacetic acid & $\mathrm{CF} 3 \mathrm{CO} 2 \mathrm{H}$ & $\mathrm{F}$ & & 238 \\
\hline Trihydrazmotriazine & & & $\mathrm{N}$ & 490 \\
\hline Trihydroxybenzene & $(\mathrm{HO}) 3 \mathrm{C} 6 \mathrm{H} 3$ & & & \\
\hline Trimethylamine & $(\mathrm{CH} 3) 3 \mathrm{~N}$ & & 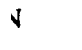 & \\
\hline Trinitrophenol & $(\mathrm{NO} 2) 3 \mathrm{C} 6 \mathrm{H} 2 \mathrm{OH}$ & & & \\
\hline Triphenylacetic acid & $(\mathrm{C} 6 \mathrm{H} 5) 3 \mathrm{CCO} 2 \mathrm{H}$ & & & \\
\hline Umbelliferone & $\mathrm{C} 9 \mathrm{H} 6 \mathrm{O} 3$ & & & \\
\hline
\end{tabular}


Table 3. Inorganic Substances Included in EPA Lists of Hazardous Substances and/or Treated by a Photocatalytic Process

\begin{tabular}{|c|c|c|}
\hline Substance/Element & Formula/Symbol & Reference \\
\hline Actinides & Th,Pa,U.Np,Pu & \\
\hline Aluminum (fume or dust) & Al & \\
\hline Aluminum oxide & A1203 & \\
\hline Ammonia & NH3 & $364,492, \mathrm{~g} 518$ \\
\hline Ammonium nitrate (soln) & NH4NO3 & 58 \\
\hline Ammonium sulfate (soln) & (NH4)2SO4 & \\
\hline Antimony & $\mathrm{Sb}$ & \\
\hline Arsenic & As & \\
\hline Asbestos & $\mathrm{Mg}, \mathrm{Si}$ & \\
\hline Azide ion & $\mathrm{N} 3(-)$ & \\
\hline Barium & $\mathrm{Ba}$ & \\
\hline Beryllium & $\mathrm{Be}$ & \\
\hline Bismuth & $\mathrm{Bi}$ & \\
\hline Boron & B & \\
\hline Cadmium & $\mathrm{Cd}$ & $221,371,372.373$ \\
\hline Chlorine & $\mathrm{Cl}$ & \\
\hline Chlorine dioxide & $\mathrm{Cl} 02$ & \\
\hline Chromium & $\mathrm{Cr}$ & $71,72,106,134,255,371,372,373$ \\
\hline Cobalt & co & \\
\hline Copper & $\mathrm{cu}$ & $\begin{array}{l}109,117,165,166,221,371,372,373 \\
198\end{array}$ \\
\hline Cyanide and Complexes & $\mathrm{CN}(-1)$ and $\mathrm{M}(\mathrm{CN}) \mathrm{x}$ & $3,9,106,178,262,377,378,379,540,545,571$ \\
\hline Cyanite ion & CNO(-1) & 57 \\
\hline Gold & $\mathrm{Au}$ & $\$ 7,115,373$ \\
\hline Halide ion & $\mathrm{X}(1-), \mathrm{X}=\mathrm{F}, \mathrm{Cl}, \mathrm{Br}$, or I & $292,398,542$ \\
\hline Hydrazine & H2NNH2 . & \\
\hline Hydrogen sulfide & $\mathrm{H} 2 \mathrm{~S}$ & $\$ 38,539,562,570$ \\
\hline Hypophosphorus acid & $\mathrm{H} 2 \mathrm{PO} 2$ & $24,163,282$ \\
\hline Iron & $\mathrm{Fe}$ & 154 \\
\hline Lead & $\mathrm{Pb}$ & 372 \\
\hline Manganese & $\mathrm{Mn}$ & \\
\hline Mercury & $\mathrm{Hg}$ & $\begin{array}{l}3,221.371,373,377,379 \\
162,499\end{array}$ \\
\hline Molybdenum & Mo & \\
\hline Nickel & $\mathrm{Ni}$ & $109.371,372,373$ \\
\hline Nitrates/nitrites & NO3(-1),NO2(-1) & :94,295 \\
\hline
\end{tabular}




\begin{tabular}{|c|c|c|}
\hline Substance/Element & Formula/Symbol & Reference \\
\hline Nitrogen oxides & Nox & $\begin{array}{l}\text { g179,g180,g181.g182,g409, } \\
\text { g410,g453 }\end{array}$ \\
\hline Nitrogen & N2 & 461 \\
\hline Oxalate ion & $\mathrm{C} 2 \mathrm{O} 4(2-)$ & \\
\hline Oxygen & 02 & \\
\hline Ozone & 03 & g436 \\
\hline Palladium & $\mathrm{Pd}$ & \\
\hline Phosphorus & & \\
\hline Platinum & $\mathrm{Pt}$ & $371,372,373$ \\
\hline Radium & $\mathrm{Ra}$ & \\
\hline Radon & Rn & \\
\hline Rhodium & $\mathrm{Rh}$ & \\
\hline Selenium & $\mathrm{Se}$ & \\
\hline Silicon & $\mathrm{Si}$ & \\
\hline Silver & $\mathrm{Ag}$ & $25,78,92,155 \ldots n \mid,-294,371,373$ \\
\hline Strontium & $\mathrm{Sr}$ & \\
\hline Sulfate radical & $504(1-)$ & \\
\hline Sulfite & & $114,294,541$ \\
\hline Sulfur & & \\
\hline Sulfur dioxide & so2 & \\
\hline Sulfuric acid & $\mathrm{H} 2 \mathrm{SO}_{4}$ & \\
\hline Thallium & $\Pi$ & \\
\hline Thiocyanate & $\mathrm{SCN}(1-)$ & 59,540 \\
\hline Thiosulfate & $\mathrm{S} 2 \mathrm{O} 3(2-)$ & \\
\hline Thorium & rh & \\
\hline Tin & Sn & \\
\hline Tritium & $\mathrm{H},(\mathrm{T})$ & \\
\hline Tungsten & W & \\
\hline Vanadium & v & \\
\hline Zinc & $\mathrm{zn}$ & \\
\hline
\end{tabular}




\subsection{Conclusions}

The level of activity in this field continues to grow at a rapid rate. The potential to develop new methods for solving environmental problems is the primary driving force for the R\&D activity. However, the patents that have been awarded and the increase in research activity in areas such as water and air disinfection, odor control, indoor air quality, and consumer products indicate that the technology has a broader commercial potential. Recent work reflects the increased interest in applying photocatalytic processes to the treatment of contaminated gas streams. More attention is also being paid to the detection and identification of intermediates and by-products that can be formed during the photocatalytic process, both in aqueous and gas phase systems. This can be an aid in developing an understanding of the chemical mechanisms of the processes and is necessary to insure that potentially harmful substances are not left in the processed stream. Still, relatively few studies include mass balances for the reactions. Kinetic models that can be used to size treatment systems are also relatively rare. As systems are deployed in the field, it is increasingly important that the issues of catalyst lifetime and regeneration be addressed. Related to this is the need to identify those components of an air or water stream that can inhibit or kill activity. All of these are important to the design of efficient and economical treatment systems. The high level of activity in this field is likely to continue -it remains to be seen how widespread the applications may be. 


\subsection{Bibliography}

1. Abe, Takeaki, inventor. "Photocatalyst." Fuji Chitan Kogyo Kk, assignee. Japan Patent, 05096181 A2. 20 April 1993. CA119(14):147490u.

modified $\mathrm{TiO}_{2}$, other semiconductor, immobilized $\mathrm{TiO}_{2}$.

2. Aguado, M. A., M. A. Anderson, and C. G. Hill Jr. "Influence of Light Intensity and Membrane Properties on the Photocatalytic Degradation of Formic Acid Over $\mathrm{TiO}_{2}$ Ceramic Membranes." J. Mol. Catal.89(1-2) (1994): 165-78.

immobilized $\mathrm{TiO}_{2}$, process efficiency, aqueous phase.

3. Aguado, M. A., S. Cervera-March, and J. Gimenez. "Continuous Photocatalytic Treatment of Mercury(II) on Titania Powders. Kinetics and Catalyst Activity." Chem. Eng. Sci. 50, no. 10 (1995): $1561-9$.

$\mathrm{TiO}_{2}$, oxidant, reductant, aqueous phase, application, mechanism, modeling, metal removal.

4. AI-Ekabi, Hussain, Brian Butters, Dale Delany, Wendy Holden, Tony Powell, and Joan Story. "The Photocatalytic Destruction of Gaseous Trichloroethylene and Tetrachloroethylene Over Immobilized Titanium Dioxide." Photocatalytic Purification and Treatment of Water and Air, eds. David F. Ollis and Hussain AI-Ekabi, 719-25, Trace Metals and the Environment, ed. Jerome 0. Nriagu, New York, NY: Elsevier, 1994.

$\mathrm{TiO}_{2}$, gas phase, immobilized $\mathrm{TiO}_{2}$.

5. Al-Ekabi, Hussain, Ali Safarzadeh-Amiri, Wendy Sifton, and Joan Story. "Advanced Technology for Water Purification by Heterogeneous Photocatalysis." Int. J. Environ. Pollut. 1, no. 1-2 (199 1): 125-36.

$\mathrm{TiO}_{2}$, reactor, aqueous phase, process efficiency, immobilized $\mathrm{TiO}_{2}$.

6. Alarcon, R. Parreno, and A. Morales-Rubio. "Online Catalytic Photodegradation of Aldicarb." J. Flow Injection Anal. 11, no. 1 (1994): 79-93.

$\mathrm{TiO}_{2}$, reactor, pesticide, aqueous phase, process efficiency.

7. Amalric, Laurence, Chantal Guillard, and Pierre Pichat. "The Photodegradation of 2,3-Benzofuran and Its Intermediates, 2-Coumaranone and Salicylaldehyde, in $\mathrm{TiO}_{2}$ Aqueous

Suspensions." J.Photochem. Photobiol., A 85, no. 3 (1995): 257-62.

$\mathrm{TiO}_{2}$, aqueous phase, application, mechanism, process efficiency.

8. Ameta, Surech C., Mukesh Mehta, Bhoopendra Sharma, and Manju Dak. "Photocatalyzed Reaction in Sodium Nitroprusside-Thiocyanate System." Izv. Vyssh. Uchebn. Zaved., Khim. Khim. Tekhnol.37, no. 2 (1994): 43-7.

$\mathrm{TiO}_{2}$, other semiconductor, aqueous phase, process efficiency, metal removal.

9. Ameta, Suresh C., Manju Bala, Jatinder Kaur, and Sapna Sahasi. "Photocatalytic Reactions of Sodium Nitroprusside." Arabian J.Sci.Eng. 19, no. 1 (1994): 71-5.

$\mathrm{TiO}_{2}$, other semiconductor, aqueous phase, application, mechanism, metal removal. 
10. Anderson, Marc A., inventor. "Reactor Process Using Metal Oxide Ceramic Membranes." Wisconsin Alumini Research Foundation, assignee. United States Patent, 5308454.3 May 1994. CA121:94509.

$\mathrm{TiO}_{2}$, other semiconductor, engineering, reactor, modified $\mathrm{TiO}_{2}$, immobilized $\mathrm{TiO}_{2}$, catalyst reactivation.

11. Anderson, Marc A., Suzuko Yamazaki-Nishida, and Salvador Cervera-March.

"Photodegradation of Trichloroethylene in the Gas Phase Using Titanium Dioxide Porous Ceramic Membrane." Photocatalytic Purification and Treatment of Water and Air, eds. David F. Ollis and Hussain Al-Ekabi, 405-20, Trace Metals and the Environment, ed. Jerome 0. Nriagu, New York, NY: Elsevier, 1994.

$\mathrm{TiO}_{2}$, reactor, gas phase, modified $\mathrm{TiO}_{2}$, immobilized $\mathrm{TiO}_{2}$.

12. Ando, Hiromi, Akiyoshi Yamaoka, Jun Yamauchi, and Masami Sugita. "Influence of Titanium Pigments on Lightfastness of Nitrocellulose (NC) Film." Hikaku Kagaku 40, no. 2 (1994): 103-10.

$\mathrm{TiO}_{2}$, application, mechanism, immobilized $\mathrm{TiO}_{2}$, polymer stabilization.

13. Anheden, Marie, D. Yogi Goswami, and G. Svedberg. "Photocatalytic Treatment of Wastewater From 5-Fluorouracil Manufacturing." Solar Engineering 1995, eds. William B. Stine, Tadayoshi Tanaka' and David E. Claridge, 439-8, New York, NY: ASME, 1995.

$\mathrm{TiO}_{2}$, oxidant' aqueous phase, application.

14. Anpo, M. "Photocatalysis and Photoinduced Reactions on Well-Defined Supported Metal Oxides - Molecular Scale Reaction Mechanisms." Photochemical Conversion and Storage of Solar Energy, Proceedings of the 8th International Conference on Photochemical Conversion and Storage of Solar Energy, eds. E. Pelizzetti and M. Schiavello, 307-2 1, Dordrecht: Kluwer Academic Publishers, 1991. $\mathrm{TiO}_{2}$, other semiconductor, modeling, gas phase, modified $\mathrm{TiO}_{2}$, mechanism, immobilized $\mathrm{TiO}_{2}$, adsorption.

15. Araz, Klodya Vert, and Miray Bekbolet. "Photochemical Sterilization of E. Coli in Drinking Water." Kim. Kim Muhendisligi Semp., Volume 4,255-K Edited by: Aydin, Adnan. Marmara Univ. Fac. Sci. Lett.: Istan, 1992.

$\mathrm{TiO}_{2}$, disinfection, aqueous phase.

16. Artem'ev, Yu. M., N. N. Nikolaeva, and M. A. Artem'eva "Some Features of the Photocatalytic Destruction of Air Pollutants - Toluene on Titanium Dioxide." Vestn. S.-Peterb. Univ., Ser. 4: Fiz., Khim. (1) (1994): 95-9.

$\mathrm{TiO}_{2}$, gas phase, oxidant, immobilized $\mathrm{TiO}_{2}$.

17. Artemjeva, M. A., and Yu. M. Artemjev. "Photostimulated Degradation of Methylene Blue in Ozonated Water Suspensions of $\mathrm{TiO}_{2}$ and $\mathrm{Nb205."} \mathrm{Vestn.} \mathrm{S.-Peterb.} \mathrm{Univ.,} \mathrm{Ser.} \mathrm{4:} \mathrm{Fit.,} \mathrm{Khim.,}$ no. 4 (1993): 90-2.

$\mathrm{TiO}_{2}$, other semiconductor, oxidant' aqueous phase.

18. Ashokkumar, M., A. Kudo, N. Saito, and T. Sakata. "Semiconductor Sensitization by RuS Colloids on $\mathrm{TiO}_{2}$ Electrodes." Chem. Phys. Let. 229 (1994): 383-K $\mathrm{TiO}_{2}$, other semiconductor, modified $\mathrm{TiO}_{2}$. 
19. Augugliaro, V., M. J. Lopez-Munoz, L. Palmisano, and J. Soria "Influence of pH on the Degradation Kinetics of Nitrophenol Isomers in a Heterogeneous Photocatalytic System." Appl.Catal., A 101, no. 1 (1993): 7-13.

$\mathrm{TiO}_{2}$, aqueous phase.

20. Augugliaro, V., G. Marci, L. Palmisano, E. Pramauro, and A. Bianco-Prevot. "Kinetics of Heterogeneous Photocatalytic Decomposition of Monuron Over Anatase Titanium Dioxide Powder." Res. Chem. Intermed. 19, no. 9 (1993): 839-53.

$\mathrm{TiO}_{2}$, pesticide, aqueous phase.

21. Augugliaro, V., M. Schiavello, and L. Palmisano. "Rate of Photoabsorption and Turnover Number: Two Parameters for the Comparison of Heterogeneous Photocatalytic Systems in a Quantitative Way." Coord. Chem. Rev. 125, no. 1-2 (1993): 173-81.

$\mathrm{TiO}_{2}$, other semiconductor, engineering, modeling, aqueous phase, process efficiency.

22. Augugliaro, Vincenzo, Vittorio Loddo, Leonardo Palmisano, and Mario Schiavello. "Performance of Heterogeneous Photocatalytic Systems: Influence of Operational Variables on Photoactivity of Aqueous Suspension." J. Catal. 153, no. 1 (1995): 3240.

$\mathrm{TiO}_{2}$, modeling, oxidant, aqueous phase, modified $\mathrm{TiO}_{2}$, process efficiency, mechanism, anion inhibition.

23. Avranas, A., I. Poulios, C. Kypri, D. Jannakoudakis, and G. Kyriakou. "Heterogeneous Photocatalytic Degradation of the Cationic Surfactant Dodecylpyridinum Chloride." Appl.Catal., B 2, no. 4 (1993): 289-302.

$\mathrm{TiO}_{2}$, other semiconductor, metallized $\mathrm{TiO}_{2}$, aqueous phase.

24. Azuma, Kunihiko, and Kenji Ootsuka, inventors. "Treatment of Aging Effluents From Plating Containing Hypophosphorous Acid Ion.” Japan Tokyo Prefecture, assignee. Japan Patent' 06136549 A2. 17 June 1994. CA121:116805.

$\mathrm{TiO}_{2}$, reductant, oxidant, metallized $\mathrm{TiO}_{2}$, aqueous phase, modified $\mathrm{TiO}_{2}$, application, engineering.

25. Baciocchi, Enrico, Cesare Rol, Giovanni V. Sebastiani, and Luca Taglieri. "Structural Effects in the $\mathrm{TiO}_{2}$-Photocatalyzed Oxidation of Alkylaromatic Compounds in Acetonitrile in the Presence of $\mathrm{Ag}_{2} \mathrm{SO}_{4} . "$ J. Org. Chem. 59, no. 18 (1994): 5272-6.

$\mathrm{TiO}_{2}$, oxidant, metallized $\mathrm{TiO}_{2}$, nonaqueous, metal removal.

26. Bahador, Sardar Khan. "Semiconducting Metal Oxide Photoelectrodes: Their Probed Characteristics and Implications. (Pt. 3). Interfacial Photodynamics." Front. Sci. Ser. 7 (New Energy Systems and Conversions) (1993): 715-20.

$\mathrm{TiO}_{2}$, aqueous phase, mechanism.

27. Bahnemann, D. W. "Mechanism of Organic Transformations on Semiconductor Particles." Photochemical Conversion and Storage of Solar Energy, Proceedings of the 8th International Conference on Photochemical Conversion and Storage of Solar Energy, eds. E. Pelizzetti and M. Schiavello, 251-76, Dordrecht: Kluwer Academic Publishers, 1991.

$\mathrm{TiO}_{2}$, other semiconductor, oxidant, aqueous phase, modified $\mathrm{TiO}_{2}$, process efficiency, mı chanism.

28. Bahnemann, Detlef. "Ultrasmall Metal Oxide Particles: Preparation, Photophysical Characterization, and Photocatalytic Properties." Isr. J. Chem.33, no. 1 (1993): 115-36.

$\mathrm{TiO}_{2}$, other semiconductor, oxidant, reductant, aqueous phase, modified $\mathrm{TiO}_{2}$, mechanism. 
29. Bai, Chuansheng. "Preparation and Characterization of the Phases Formed by the Reactions of Transition Metal Precursors With $\mathbf{M g O}, \mathrm{Al}_{2} \mathrm{O}_{3}$, and $\mathrm{MgAl}_{2} \mathrm{O}_{4}$, and Preparation and Characterization of Titanium(N) Oxide Photocatalysts." Ph.D. diss., Brown Univ., Providence, RI, USA, 1993.

$\mathrm{TiO}_{2}$, modified $\mathrm{TiO}_{2}$.

30. Bajt, O., B. Sket, and J. Faganeli. "The Effect of Semiconductor Oxides on the Photochemical Degradation of Phthalic and Maleic Anhydrides in Aqueous Media." Toxicol. Environ. Chem. 40, no. 1-4 (1993): 267-73.

$\mathrm{TiO}_{2}$, other semiconductor, aqueous phase.

31. Bamwenda, G. R., S. Tsubota, T. Kobayashi, and M. Haruta "Photoinduced Hydrogen Production From an Aqueous Solution of Ethylene Glycol Over Ultrafine Gold Supported on $\mathrm{TiO}_{2}$." J. Photochem. Photobiol. A: Chem. 77 (1994): 59-67.

$\mathrm{TiO}_{2}$, reductant, metallized $\mathrm{TiO}_{2}$, aqueous phase, modified $\mathrm{TiO}_{2}$.

32. Bard, Allen J. "Photoelectrochemistry and Heterogeneous Photocatalysis at Semiconductors." J. Photochem. 10 (1979): 59-75.

$\mathrm{TiO}_{2}$, other semiconductor, reactor, modeling, solar, metallized $\mathrm{TiO}_{2}$, aqueous phase, modified $\mathrm{TiO}_{2}$, application, process efficiency, mechanism.

33. Bard, Allen J. "Semiconductor Particles and Arrays for the Photoelectrochemical Utilization of Solar Energy." Ber. Bunsenges. Phys. Chem. 92 (1988): 1187-94.

$\mathrm{TiO}_{2}$, other semiconductor, solar, aqueous phase, application, mechanism.

34. Barreto, Reynaldo D., Kimberly A. Gray, and Krista Anders. "Photocatalytic Degradation of Methyl-Tert-Butyl Ether in TiO $_{2}$ Slurries: a Proposed Reaction Scheme." Water Res. 29, no. 5 (1995): $1243-8$

$\mathrm{TiO}_{2}$, aqueous phase, application, mechanism.

35. Beaune, O., A. Finiels, P. Geneste, P. Graffin, A. Guida, J. L. Olive, and A. Saeedan. "Selective Photocatalytic Oxidation of Hydrocarbon Compounds Over Zeolites." Stud. Surf. Sci. Catal. 78, Heterogeneous Catalysis and Fine Chemicals III (1993): 401-8.

$\mathrm{TiO}_{2}$, modified $\mathrm{TiO}_{2}$, gas phase, immobilized $\mathrm{TiO}_{2}$, adsorbent.

36. Becker, M., B. Gupta, W. Meineke, and M. Bohn. Solar Energy Concentrating Systems. Heidelberg: C. F. Muller Verlag, 1995.

engineering, solar, reactor.

37. Bedford, J., James F. Klausner, D. Yogi Goswami, and Kirk S. Schanze. "Performance of Nonconcentrating Solar Photocatalytic Reactors: Part I - Shallow Pond Configuration." Solar Engineering 1993, eds. Allan Kirkpatrick, and William Worek, 3542, New York, NY: ASME, 1993.

aqueous phase, engineering, solar, $\mathrm{TiO}_{2}$.

38. Bedja, Idriss, Surat Hotchandani, and Prashant K. Kamat. "Preparation and Photoelectrochemical Characterization of Thin Sn02 Nanocrystalline Semiconductor Films and Their Sensitization With Bis(2,2'-Bipyridine-4,4'-Dicarboxylic Acid)Ruthenium(II) Complex." J. Phys. Chem. 98, no. 15 (1994): 4133-O.

other semiconductor. 
39. Bedja, Idriss, Surat Hotchandani, and Prashant V. Kamat. "Photoelectrochemistry of Quantized $\mathrm{WO}_{3}$ Colloids. Electron Storage, Electrochromic, and Photoelectrochromic Effects." J. Phys. Chem. 97, no. 42 (1993): 11064-70.

other semiconductor, reductant, aqueous phase, mechanism.

40. Bellobono, Ignazio R., Carrara Anna, Barbara Bami, and Aleardo Gazzotti. "Laboratoryand Pilot-Plant-Scale Photodegradation of Chloroaliphatics in Aqueous Solution by Photocatalytic Membranes Immobilizing Titanium Dioxide." J. Photochem. Photobiol A: Chem. 84 (1994): 83-90. $\mathrm{TiO}_{2}$, other semiconductor, engineering, modeling, aqueous phase, modified $\mathrm{TiO}_{2}$, immobilized $\mathrm{TiO}_{2}$.

41. Bellobono, Ignazio Renato, and Anna Carrara "Laboratory and Pilot Reactor Experience for Photochemical Degradation of Organic Contaminants in Wastewaters by Photocatalytic Membranes Immobilizing Titanium Dioxide.” BHR Group Conf. Ser. Publ. 3, Effective Membrane Processes-New Perspectives (1993): 257-74.

aqueous phase, immobilized $\mathrm{TiO}_{2}$.

42. Bennett, P. A., and S. Beadles. "Photocatalytic Oxidation: a Safe and Clean Alternative in TOC Analysis." Am. Lab. 26, no. 15 (1994): 29-32.

$\mathrm{TiO}_{2}$, aqueous phase, application, immobilized $\mathrm{TiO}_{2}$.

43. Berman, Elliot, and Junchang Dong. "Photocatalytic Decomposition of Organic Pollutants in Gas Streams." Chem.Oxid. Volume Date 1993, no. 3 (1994): 183-9.

$\mathrm{TiO}_{2}$, gas phase, modified $\mathrm{TiO}_{2}$, application, process efficiency, immobilized $\mathrm{TiO}_{2}$, solar.

44. Berry, R. James, and Michael R. Mueller. "Photocatalytic Decomposition of Crude Oil Slicks Using $\mathrm{TiO}_{2}$ on a Floating Substrate." Microchem. J. 50, no. 1 (1994): 28-32.

$\mathrm{TiO}_{2}$, solar, aqueous phase, modified $\mathrm{TiO}_{2}$, immobilized $\mathrm{TiO}_{2}$, adsorption.

45. Bickley, R. I. "Heterogeneous Photo-Catalysis.“, 308-2. Catalysis, A Specialist Periodical Report, eds. G. C. Bond, and G. Webb, 5. London: The Chemical Society, 1982.

$\mathrm{TiO}_{2}$, other semiconductor, solar, gas phase, aqueous phase.

46. Bickley, R. I., G. Munuera, and F. S. Stone. "Photoadsorption and Photocatalysis At Rutile Surfaces. II. Photocatalytic Oxidation of Isopropanol.” J. Catal. (1973): 398-407. gas phase, modified $\mathrm{TiO}_{2}$, $\mathrm{TiO}_{2}$.

47. Bickley, R. I., L. Palmisano, M. Schiavello, and A. Sclafani. "Heterogeneous Photocatalysis: Mechanistic Considerations of Photocatalytic Reductions and Photocatalytic Oxidations on Semiconductor Oxide Surfaces." Stud. Surf. Sci. Catal. 75, New Frontiers in Catalysis, Pt. C (1993): 2151-4.

$\mathrm{TiO}_{2}$, aqueous phase, modified $\mathrm{TiO}_{2}$.

48. Bickley, R. I., and F. S. Stone. "Photoadsorption and Photocatalysis At Rutile Surfaces. I. Photoadsorption of Oxygen." J. Catal. 31 (1973): 389-97.

adsorption, gas phase, $\mathrm{TiO}_{2}$, oxidant.

49. Bickley, Roger I., Teresita Gonzalez-Carreno, Agustin R. Gonzalez-Elipe, Guillermo Munuera, and Leonardo Palmisano. "Characterization of Iron,Titanium Oxide Photocatalysts. Part 2. Surface Studies.” J.Chem. Soc., Faraday Trans. 90, no. 15 (1994): 2257-64, 1 plate. $\mathrm{TiO}_{2}$, modified $\mathrm{TiO}_{2}$. 
50. Bideau, M., B. Claudel, L. Faure, and H. Kazouan. "Metallic Complexes As Intermediates in Homogeneously and Heterogeneously Photocatalysed Reactions." J. Photochem. Photobiol. A: Chem. 84 (1994): 57-67.

$\mathrm{TiO}_{2}$, other semiconductor, modeling, aqueous phase, mechanism.

51. Blake, Daniel M. "Solar Processes for the Destruction of Hazardous Chemicals." Alternative Fuels and the Environment, ed. Francis S. Sterrett, 175-86. Boca Raton, FL: Lewis Publishers, 1994.

$\mathrm{TiO}_{2}$, engineering, solar, gas phase, aqueous phase, application.

52. Blanco, Julian, and Sixto Malato. "Wastewater Decontamination by Solar Photocatalysis." Ing. Quim. 25, no. 286 (1993): 129-37.

$\mathrm{TiO}_{2}$, engineering, reactor, solar, aqueous phase, application, adsorbent.

53. Blanco, Julian, and Sixto Malato. "Solar Photocatalytic Mineralization of Real Hazardous Waste Water At Pre-Industrial Level."Solar Engineering 1994, eds. David E. Klett, Roy E. Hogan, and Tadayoshi Tanaka' 103-9, 1994.

$\mathrm{TiO}_{2}$, engineering, reactor, solar, oxidant' aqueous phase, application, cost.

54. Blazkova, A., V. Brezova, Z. Soldanova, A. Stasko, M. Soldan, and M. Ceppan.

"Photocatalytic Degradation of Heparin Over Titanium Dioxide." J. Mater. Sci. 30, no. 3 (1995): 729-33. . $\mathrm{TiO}_{2}$, aqueous phase, application, mechanism.

55. Block, Seymour S., and D. Yogi Goswami. "Chemically Enhanced Sunlight for Killing Bacteria." Solar Engineering 1995, eds. William B. Stine, Tadayoshi Tanaka' and David E. Claridge, 431-7, New York, NY: ASME, 1995.

$\mathrm{TiO}_{2}$, solar, disinfection, aqueous phase.

56. Bolton, J. R., A. Safarzadeh-Amiri, and S. R. Cater. "The Detoxification of Waste Water Streams Using Solar and Artificial Light Sources." Alternative Fuels and the Environment, ed. Francis S. Sterrett, 187-92. Boca Raton, FL: Lewis Publishers, 1995. aqueous phase, cost, process efficiency, $\mathrm{TiO}_{2}$, solar.

57. Bravo, Agustin, Josep Garcia' Xavier Domenech, and Jose Peral. "Some Observations About the Photocatalytic Oxidation of Cyanate to Nitrate Over TiO." Electrochim. Acta 39, no. 16 (1994): 2461-3.

$\mathrm{TiO}_{2}$, aqueous phase, mechanism.

58. Bravo, Agustin, Josep Garcia' Xavier Domenech, and Jose Peral. "Some Aspects of the Photocatalytic Oxidation of Ammonium Ion by Titanium Dioxide." J. Chem. Res., Synop., no. 9 (1993): 376-7.

$\mathrm{TiO}_{2}$, aqueous phase, adsorption.

59. Brezova, V., M. Breza, and M. Ceppan. "The Photocatalytic Degradation of Cyclic Acetals in Aqueous Titanium Dioxide Suspension." Chem. Pap. 46, no. 6 (1992): 359-63.

$\mathrm{TiO}_{2}$, aqueous phase, mechanism. 
60. Brezova, V., M. Jankovicova, M. Soldan, A. Blazkova, M. Rehakova, I. Surina, M. Ceppan, and B. Havlinova. "Photocatalytic Degradation of P-Toluenesulfonic Acid in Aqueous Systems Containing Powdered and Immobilized Titanium Dioxide." J. Photochem. Photobiol., A 83, no. 1 (1994): 69-75.

$\mathrm{TiO}_{2}$, oxidant, aqueous phase, modified $\mathrm{TiO}_{2}$, immobilized $\mathrm{TiO}_{2}$.

61. Brezova, V., and A. Stasko. "Spin Trap Study of Hydroxyl Radicals Formed in the Photocatalytic System $\mathrm{TiO}_{2}$-Water-p-Cresol-Oxygen." J. Catal. 147, no. 1 (1994): 156-62.

$\mathrm{TiO}_{2}$, modeling, aqueous phase, process efficiency, mechanism, adsorption.

62. Brezova, V., A. Stasko, A. Blazkova, and B. Havlinova "Kinetics of Hydroxyl Radical Spin Trapping in Photoactivated Homogeneous $\left(\mathrm{H}_{2} \mathrm{O}_{2}\right)$ and Heterogeneous $\left(\mathrm{TiO}_{2}\right)$ Aqueous Systems." $J$. Phys. Chem. 98, no. 36 (1994): 8977-4.

$\mathrm{TiO}_{2}$, aqueous phase, mechanism, adsorption.

63. Brezova, V., A. Stasko, M. Ceppan, M. Mikula, J. Blecha, M. Vesely, A. Blazkova, J. Panak, and L. Lapcik. "Photocatalytic Activity of Titanium Dioxide and the Formation of Radical Intermediates." Photocatalytic Purification and Treatment of Water and Air, eds. David F. Ollis and Hussain Al-Ekabi, 659-4, Trace Metals and the Environment, ed. Jerome 0. Nriagu, New York, NY: Elsevier, 1994.

$\mathrm{TiO}_{2}$, mechanism.

64. Brun, H., J. P. Percherancier, and B. Pouyet. "Study of Optimal Experimental Conditions in the Photocatalytic Degradation of an Herbicide." Environ. Technol. 16, no. 4 (1995): 395-400. $\mathrm{TiO}_{2}$, pesticide, aqueous phase, process efficiency.

65. Brym, Stanley J., inventor. "Water Purification System." Conventure Corp, assignee. United States patent, 5227053. 13 July 1993. CA119(22):233639f.

$\mathrm{TiO}_{2}$, other semiconductor, reactor, disinfection, aqueous phase, application, immobilized $\mathrm{TiO}_{2}$, adsorbent.

66. Butler, Elizabeth C., and Allen P. Davis. "Photocatalytic Oxidation in Aqueous Titanium Dioxide Suspensions: the Influence of Dissolved Transition Metals." J. Photochem. Photobiol., A 70, no. 3 (1993): 273-83.

$\mathrm{TiO}_{2}$, oxidant, aqueous phase, mechanism.

67. Caballero, A., A. R. Gonzalez-Elipe, A. Fernandez, J.-M. Herrmann, H. Dexpert, and F. Villain. "Experimental Set-Up for in-Situ X-Ray Absorption Spectroscopy Analysis of Photochemical Reactions: the Photocatalytic Reduction of Gold on Titania." J. Photochem. Photobiol., A 78, no. 2 (1994): $169-72$.

$\mathrm{TiO}_{2}$, oxidant, metallized $\mathrm{TiO}_{2}$.

68. Cabrera, Maria I., Orlando M. Alfano, and Alberto E. Cassano. "Novel Reactor for Photocatalytic Kinetic Studies.” Ind. Eng. Chem. Res. 33, no. 12 (1994): 3031-42. $\mathrm{TiO}_{2}$, reactor, modeling, aqueous phase, process efficiency.

69. Caruana, Claudia M. "Photocatalysts Aim to Make Light Work of Pollution Cleanup." Chem. Eng. Prog. 91, no. 2 (1995): 11-20.

$\mathrm{TiO}_{2}$, engineering, gas phase, aqueous phase, application, process efficiency. 
70. Catilaz, L., and J. P. Fouassier. "Role of Pigments in Polymerization Under UV-Visible Light." Angew. Makromol. Chem. 218 (1994): 81-109.

71. Cervera-March, S., J. Gimenez-Farreras, M. A. Aguado, L. Borrell, D. Curco, and M. A. Queral. "Kinetic and Radiation Studies for the Photoreactor Design in Photocatalytic Detoxification Processes Using Titanium Dioxide." Photocatalytic Purification and Treatment of Water and Air, eds. David F. Ollis and Hussain Al-Ekabi, 633-8, Trace Metals and the Environment, ed. Jerome 0. Nriagu, New York, NY: Elsevier, 1994.

$\mathrm{TiO}_{2}$, modeling, aqueous phase, process efficiency, metal removal.

72. Cervera, S., J. Gimenez, and D. Curco. "Reaction Rate and Radiation Absorption in Photocatalysis." Proc. - Electrochem. Soc. 94-19(Water Purification by Photocatalytic Photoelectrochemical, and Electrochemical Processes) (1994): 255-66. $\mathrm{TiO}_{2}$, modeling, solar, reactor, oxidant, aqueous phase, process efficiency, metal removal.

73. Chant, Eileen E., Carmen A. Ortiz-Aponte, and Maribel Navarro-Ortiz. "Photocatalytic Detoxification of Tetrahydrofuran and Chloroform in Dilute Aqueous Solutions. " Solar Engineering 1995, eds. William B. Stine, Tadayoshi Tanaka, and David E. Claridge, 475-84, New York, NY: ASME, 1995. $\mathrm{TiO}_{2}$, oxidant, aqueous phase, application.

74. Chen, Ci-Ping, Xin-Min Ren, Dao-Hui Lu, and Guang-Zhi Xu. "Study on Free Radicals Produced During the Photolysis of p-Aminophenol in Aqueous $\mathrm{TiO}_{2}$ Suspension." Chin. Sci. Bull. 39, no. 1 (1994): 41-6. aqueous phase, $\mathrm{TiO}_{2}$, mechanism.

75. Chen, Ciping, Daohui $\mathrm{Lu}$, and Guangzhi Xu. "Free Radicals Generated in Photocatalytic Oxidation of Some Organic Compounds Containing Nitrogen Atoms." J. Environ. Sci 5, no. 4 (1993): 464-9.

$\mathrm{TiO}_{2}$, aqueous phase, mechanism.

76. Chen, H. Y., 0. Zahraa, M. Bouchy, F. Thomas, and J. Y. Bottero. "Adsorption Properties of $\mathrm{TiO}_{2}$ Related to the Photocatalytic Degradation of Organic Contaminants in Water." J. Photochem. Photobiol., A 85, no. 1-2 (1995): 179-86.

$\mathrm{TiO}_{2}$, modeling, aqueous phase, adsorption.

77. Chen, Lung Chyuan, and Tse-Chuan Chou. "Photobleaching of Methyl Orange in Titanium Dioxide Suspended in Aqueous Solution." J. Mol.Catal. 85, no. 2 (1993): 201-14. $\mathrm{TiO}_{2}$, aqueous phase, process efficiency.

78. Chen, Lung-Chyuan, and Tse-Chuan Chou. "Photodecolorization of Methyl Orange Using Silver Ion Modified $\mathrm{TiO}_{2}$ As Photocatalyst." Ind. Eng. Chem. Res. 33, no. 6 (1994): 143643. $\mathrm{TiO}_{2}$, oxidant, aqueous phase, process efficiency, adsorption.

79. Chen, Paris Honglay, and Christina H. Jeng. "Kinetic Study of Photocatalytic Oxidation With $\mathrm{TiO}_{2}$ to Remove DOC in \&inking Water." Zhongguo Huanjing Gongcheng Xuekun 4, no. 2 (1994): $79-87$.

$\mathrm{TiO}_{2}$, aqueous phase, application, adsorption, modeling. 
80. Chester, Cordon, Marc A. Anderson, Harry Read, and Santiago Esplugas. "A Jacketed Annular Membrane Photocatalytic Reactor for Wastewater Treatment: Degradation of Formic Acid and Atrazine." J.Photochem. Photobiol., A 71, no. 3 (1993): 291-7. $\mathrm{TiO}_{2}$, aqueous phase, modified $\mathrm{TiO}_{2}$, immobilized $\mathrm{TiO}_{2}$, modeling, process efficiency.

81. Choi, Wonyong, Andreas Termin, and Michael R. Hoffmann. "Effect of Doped Metal Ions on the Photocatalytic Reactivity of $\mathrm{TiO}_{2}$ Quantum Particles." Angew. Chem. 106, no. 10 (1994): 1148-9. $\mathrm{TiO}_{2}$, aqueous phase, modified $\mathrm{TiO}_{2}$.

82. Choi, Wonyong, Andreas Termin, and Michael R. Hoffmann. "The Role of Metal Ion Dopants in Quantum-Sized $\mathrm{TiO}_{2}$ : Correlation Between Photoreactivity and Charge Carrier Recombination Dynamics." J. Phys. Chem. 98, no. 51 (1994): 13669-79.

$\mathrm{TiO}_{2}$, modeling, aqueous phase, modified $\mathrm{TiO}_{2}$, process efficiency, mechanism.

83. Christensen, P. A., A. Hamnett, R. He, C. R. Howarth, and K. E. Shaw. "Fundamental Photocatalytic Studies on Immobilized Films of Titanium Dioxide." Photocatalytic Purification and Treatment of Water and Air, eds. David F. Ollis and Hussain Al-Ekabi, 765-70, Trace Metals and the Environment, ed. Jerome 0. Nriagu, New York, NY: Elsevier, 1994.

$\mathrm{TiO}_{2}$, immobilized $\mathrm{TiO}_{2}$, gas phase.

84. Christensen, P. A., A. Hamnett, R. He, C. R. Howarth, and K. E. Shaw. "Photocatalytic Detoxification of Water." Spec.Publ.-R Soc.Chem. 146 (Electrochemistry and Clean Energy) (1994): 64-86.

$\mathrm{TiO}_{2}$, mechanism, aqueous phase, immobilized $\mathrm{TiO}_{2}$.

85. Cooper, Gerald, and Matthew A. Ratcliff, inventors. "Method of Decontaminating a Contaminated Fluid by Using Photocatalytic Particles." Photocatalytics, Inc. assignee. United States Patent, 52943 15.15 March 1994.

$\mathrm{TiO}_{2}$, other semiconductor, engineering, reactor, gas phase, aqueous phase, application, adsorption.

86. Courbon, H., Marc Formenti, and Pierre Pichat. "Study of Oxygen Isotopic Exchange Over Ultraviolet Irradiated Anatase Samples and Comparison with the Photooxidation of Isobutane to Acetone." J. Phys. Chem. 81, no. 6 (1977): 550-4.

$\mathrm{TiO}_{2}$, gas phase, mechanism.

87. Crittenden, John C., Sawang Notthakun, David W. Hand, and David L. Perram, inventors. "Regeneration of Adsorbents Using Advanced Oxidation." Michigan Technological University, assignee. United States Patent, 5182030.26 January 1993. CAl19(6):52272k.

$\mathrm{TiO}_{2}$, other semiconductor, reactor, metallized $\mathrm{TiO}_{2}$, aqueous phase, modified $\mathrm{TiO}_{2}$, application, immobilized $\mathrm{TiO}_{2}$, adsorbent.

88. Crittenden, John C., Yin Zhang, David W. Hand, and David L. Perram. 'Destruction of Organic Compounds in Water Using Fixed-Bed Photocatalysts." Solar Engineering 1995, eds. William B. Stine, Tadayoshi Tanaka, and David E. Claridge, 449-57, New York, NY: ASME, 1995. $\mathrm{TiO}_{2}$, solar, modeling, metallized $\mathrm{TiO}_{2}$, aqueous phase, modified $\mathrm{TiO}_{2}$, application, process efficiency, immobilized $\mathrm{TiO}_{2}$, adsorption.

89. Cui, H., K. Dwight, S. Soled, and A. Wold. "Surface Acidity and Photocatalytic Activity of $\mathrm{Nb}_{2} \mathrm{O}_{5}, \mathrm{TiO}_{2}$ Photocatalysts." J. Solid State Chem. 115, no. 1 (1995): 187-91.

$\mathrm{TiO}_{2}$, aqueous phase, modified $\mathrm{TiO}_{2}$, adsorption. 
90. Cunningham, J. "Radiation and Photoeffects At Gas,Solid Interfaces." Simple Processes At the Gas-Solid Interface, 291-427. Comprehensive Chemical Kinetics, eds. C. H. Bamford, C. F. H. Tipper, and R. G. Compton, 19. New York: Elsevier,' 1984.

$\mathrm{TiO}_{2}$, other semiconductor, modeling, metallized $\mathrm{TiO}_{2}$, gas phase, mechanism, adsorbent, adsorption.

91. Cunningham, Joseph, and Petr Sedlak. "Initial Rates of Titanium Dioxide-Photocatalyzed Degradations of Water Pollutants: Influences of Adsorption, $\mathrm{pH}$ and Photon-Flux." Photocatalytic Purification and Treatment of Water and Air, eds. David F. Ollis and Hussain Al-Ekabi, 67-8 1, Trace Metals and the Environment, ed. Jerome 0. Nriagu, New York, NY: Elsevier, 1994.

$\mathrm{TiO}_{2}$, modeling, aqueous phase, mechanism, adsorption.

92. Cunningham, Joseph, and Petr Sedlak. "Interactive Adsorption and Charge-Transfer Processes in UV-Illuminated Aqueous Pollutant, $\mathrm{TiO}_{2}$ Slurries." Proc. Electrochem. Soc. 94-19(Water Purification by Photocatalytic Photoelectrochemical, and Electrochemical Processes) (1994): 267-77. $\mathrm{TiO}_{2}$, modeling, oxidant, aqueous phase, mechanism, adsorption.

93. D'Oliveira, J. C., W. D. W. Jayatilake, K. Tennakone, J. M. Herrmann, and P. Pichat. "Heterogeneous Photocatalysis As a Method of Water Decontamination: Degradation of 2-,3-, and 4-Chlorobenzoic Acids Over Illuminated Titania At Room Temperature." Stud. Surf. Sci. Catal. 75, no. New Frontiers in Catalysis, Pt. C (1993): 2167-7 1.

$\mathrm{TiO}_{2}$, aqueous phase, mechanism.

94. Dagan, Geula, Srinivasan Sampath, and Ovadia Lev. "Preparation and Utilization of Organically Modified Silica-Titania Photocatalysts for Decontamination of Aquatic Environments." Chem. Mater. 7, no. 3 (1995): 446-53.

$\mathrm{TiO}_{2}$, aqueous phase, modified $\mathrm{TiO}_{2}$, application, mechanism.

95. Dagan, Geula, and Micha Tomkiewicz. 'Preparation and Characterization of $\mathbf{T i O}_{2}$ Aerogels for Use As Photocatalysts.” J. Non-Cryst. Solids 175, no. 2,3 (1994): 294-302. $\mathrm{TiO}_{2}$, aqueous phase, modified $\mathrm{TiO}_{2}$, adsorption.

96. Dagan, Geula, and Micha Tomkiewicz. "Preparation and Utilization of Porous Titanium Dioxide for Photocatalysis in Aquatic Environments." Proc. - Electrochem. Soc. 93-18, Proceedings of the Symposium on Environmental Aspects of Electrochemistry and Photochemistry (1993): 137-46. $\mathrm{TiO}_{2}$, aqueous phase, modified $\mathrm{TiO}_{2}$, immobilized $\mathrm{TiO}_{2}$.

97. Dagan, Geula, and Micha Tomkiewicz. "Titanium Dioxide Aerogels for Photocatalytic Decontamination of Aquatic Environments." J. Phys. Chem. 97, no. 49 (1993): 1265 1-5. $\mathrm{TiO}_{2}$, aqueous phase, modified $\mathrm{TiO}_{2}$, immobilized $\mathrm{TiO}_{2}$.

98. Das, S., M. Muneer, and K. R. Gopidas. "Photocatalytic Degradation of Wastewater Pollutants. Titanium Dioxide-Mediated Oxidation of Polynuclear Aromatic Hydrocarbons." J. Photochem. Photobiol., A 77, no. 1 (1994): 83-8.

$\mathrm{TiO}_{2}$, solar, oxidant, mechanism.

99. Datye, Abhaya K., Georg Riegel, James R. Bolton, Min Huang, and Michael R. Prairie. "Microstructural Characterization of a Fumed Titanium Dioxide Photocatalyst." J. Solid State Chem. 115, no. 1 (1995): 236-9.

$\mathrm{TiO}_{2}$, aqueous phase, catalyst characterization. 
100. Davis, Robert J., John L. Gainer, Gilbert O'Neal, and I Wen Wu. "Photocatalytic Decolorization of Wastewater Dyes." Water Environ. Res. 66, no. 1 (1994): 50-3.

$\mathrm{TiO}_{2}$, aqueous phase, application.

101. de la Guardia' Miguel, Karim D. Khalaf, Berween A. Hasan, Angel Morales-Rubio, and Vicente Carbonell. "In-Line, Titanium Dioxide-Catalyzed, Ultraviolet Mineralization of Toxic Aromatic Compounds in the Waste Stream From a Flow-Injection-Based Resorcinol Analyzer." Analyst 120, no. 2 (1995): 23 1-5.

$\mathrm{TiO}_{2}$, aqueous phase, application.

102. Dellinger, Barry, John L. Graham, Joel M. Berman, and Zhen Jiang. "Photocatalytically Enhanced Oxidation of Chlorinated Hydrocarbons." Combust. Fundam. Appl., Jt. Tech. Meet., Cent. East. States Sect. Combust. Inst., 448-52, Combustion Inst. 1993.

$\mathrm{TiO}_{2}$, solar, gas phase, mechanism, immobilized $\mathrm{TiO}_{2}$.

103. Dieckmann, Melissa S., Kimberly A. Gray, and Richard G. Zepp. "The Sensitized Photocatalysis of Azo Dyes in a Solid System: a Feasibility Study." Chemosphere 28, no. 5 (1994): 1021-34.

Dyes, $\mathrm{TiO}_{2}$, other semiconductor, application, immobilized $\mathrm{TiO}_{2}$, adsorption.

104. Do, Y. R, W. Lee, K. Dwight' and A. Wold. "The Effect of $\mathbf{W O}_{3}$ on the Photocatalytic Activity of $\mathrm{TiO}_{2}$." J. Solid State Chem. 108, no. 1 (1994): 198-201. $\mathrm{TiO}_{2}$, aqueous phase, modified $\mathrm{TiO}_{2}$, mechanism.

105. Do, Youngrag. "The Crystal Growth and Characterization of I-III-VI2-Doped II-VI Chalcogenides and the Effect of WO, on the Photocatalytic Activity of $\mathrm{TiO}_{2}$." Ph.D. diss., Brown Univ., USA, 1994.

$\mathrm{TiO}_{2}$, modified $\mathrm{TiO}_{2}$, catalyst characterization.

106. Domenech, X. "Photocatalysis for Aqueous Phase Decontamination: Is Titanium Dioxide the Better Choice?" Photocatalytic Purification and Treatment of Water and Air, eds. David F. Ollis and Hussain Al-Ekabi, 337-51, Trace Metals and the Environment, ed. Jerome 0. Nriagu, New York, NY: Elsevier, 1994.

$\mathrm{TiO}_{2}$, other semiconductor, aqueous phase, metal removal.

107. Dong, C. D., and C. P. Huang. "A Comparative Study on the Direct Photolysis and Titanium Dioxide-Mediated Photodegradation of 2-Chlorophenol in Aqueous Solutions." Photocatalytic Purification and Treatment of Water and Air, eds. David F. Ollis and Hussain Al-Ekabi, 701-6, Trace Metals and the Environment' ed. Jerome 0. Nriagu, New York, NY: Elsevier, 1994. $\mathrm{TiO}_{2}$, aqueous phase, mechanism.

108. Dong, Chengdi, and Chin-Pao Huang. "Photocatalytic Degradation of 4-Chlorophenol in $\mathrm{TiO}_{2}$ Aqueous Suspensions." Adv. Chem. Ser. 244(Aquatic Chemistry) (1995): 291-3 13. $\mathrm{TiO}_{2}$, aqueous phase, mechanism.

109. Dong, Jim J., and Ronald J. Enzweiler. "Reductive Photocatalysis: A New Separation Technology for Heavy Metals." Adv. Filtr. Sep. Technol. 8 (1994): 283-6.

$\mathrm{TiO}_{2}$, oxidant, metal removal. 
110. Enzweiler, Ron J., Debby L. Mowery, Larry M. Wagg, and Jim J. Dong. "A Pilot Scale Investigation of Photocatalytic Detoxification of BETX in Water." Solar Engineering 1994, eds. David E. Klett, Roy E. Hogan, and Tadayoshi Tanaka' 155-62, 1994.

$\mathrm{TiO}_{2}$, engineering, reactor, solar, oxidant, aqueous phase, application, immobilized $\mathrm{TiO}_{2}$, cost.

111. Enzweiler, Ron, Wagg, Larry, and Dong, Jim. "Requirement, Design and Field Test of State of-the-Art Industrial Wastewater Recycling System." Presented at AIChE Summer Meeting, Seattle, WA, 17 August 1993, 28 pp.

112. Evans, J. E., K. W. Springer, and J. Z.Zhang. "Femtosecond Studies of Interparticle Electron Transfer in a Coupled CdS-TiO 2 Colloidal System." J. Chem. Phys. 101, no. 7 (1994): 6222-5. $\mathrm{TiO}_{2}$, other semiconductor, mechanism, process efficiency.

113. Fan, Jingfu, and John T. Yates Jr. "Infrared Study of the Oxidation of Hexafluoropropene on $\mathrm{TiO}_{2}$." J. Phys. Chem. 98 (1994): 10621-7.

$\mathrm{TiO}_{2}$, gas phase, mechanism, thermal catalysis.

114. Faust, Bruce C., Michael R. Hoffmann, and Detlef W. Bahnemann. "Photocatalytic Oxidation of Sulfur Dioxide in Aqueous Suspensions of Alpha-Fe O $_{3}$." J. Phys. Chem. 93, no. 17 (1989): 6371-81.

other semiconductor, aqueous phase, process efficiency.

115. Femandez, A., A. Caballero, A. R. Gonzalez-Elipe, J.-M. Herrmann, H. Dexpert, and F. Villain. "In Situ EXAFS Study of the Photocatalytic Reduction and Deposition of Gold on Colloidal Titania." J. Phys. Chem. 99, no. 10 (1995): 3303-9.

$\mathrm{TiO}_{2}$, reductant, metallized $\mathrm{TiO}_{2}$, aqueous phase, mechanism, metal removal.

116. Formenti, M., and S. J. Teichner. "Heterogeneous Photo-Catalysis." 87-106. Catalysis, A Specialist Periodical Report, eds. C. Kemball and D. A. Dowden, 2. London: The Chemical Society, 1978. $\mathrm{TiO}_{2}$, other semiconductor, solar, gas phase, aqueous phase.

117. Foster, Nancy S., Garrett N. Brown, Richard D. Noble, and Carl A. Koval. "Use of Titanium Dioxide Photocatalysis in the Treatment of Spent Electroless Copper Plating Baths." Photocatalytic Purification and Treatment of Water and Air, eds. David F. Ollis and Hussain Al-Ekabi, 365-73, Trace Metals and the Environment, ed. Jerome 0. Nriagu, New York, NY: Elsevier, 1994. $\mathrm{TiO}_{2}$, engineering, aqueous phase, metal removal.

118. Foster, Nancy S., Carl A. Koval, and Richard D. Noble, inventors. "Reversible Photodeposition and Dissolution of Metal Ions." USA University of Colorado, assignee. United States Patent, 5332508.26 July 1994. CA121:140869.

$\mathrm{TiO}_{2}$, other semiconductor, aqueous phase, application, metal removal.

119. Fotou, George P., Srinivas Vemury, and Sotiris E. Pratsinis. "Synthesis and Evaluation of Titania Powders for Photodestruction of Phenol." Chem. Eng. Sci 49, no. 24B (1994): 4939-8. $\mathrm{TiO}_{2}$, aqueous phase, modified $\mathrm{TiO}_{2}$.

120. Fox, Marye Anne, Kate E. Doan, and Maria T. Dulay. "The Effect of the "Inert" Support on Relative Photocatalytic Activity in the Oxidative Decomposition of Alcohols on Irradiated Titanium Dioxide Composites." Res. Chem. Intermed. 20, no. 7 (1994): 71 1-22.

nonaqueous, $\mathrm{TiO}_{2}$, modified $\mathrm{TiO}_{2}$, process efficiency, immobilized $\mathrm{TiO}_{2}$, adsorbent. 
121. Fox, Marye Anne, R. Barton Draper, Maria Dulay, and Kevin O'Shea. "Control of Photocatalytic Oxidative Selectivity on Irradiated $\mathrm{TiO}_{2}$ Powders: Diffuse Reflectance Kinetic Study." Photochemical Conversion and Storage of Solar Energy, Proceedings of the 8th International Conference on Photochemical Conversion and Storage of Solar Energy, eds. E. Pelizzetti, and M. Schiavello, 323-5, Dordrecht: Kluwer Academic Publishers, 1991.

nonaqueous, $\mathrm{TiO}_{2}$, mechanism.

122. Fox, Marye Anne, Shiyamalie Ruberu, Andrew Hadd, and Young Soo Kim. "Competitive Photooxidative Degradation of Amines and Alcohols on Heterogeneously Suspended Titanium Dioxide Particles." Proc.- Electrochem. Soc. 93-18, no. Proceedings of the Symposium on Environmental Aspects of Electrochemistry and Photoelectrochemistry (1993): 104-1.

nonaqueous, $\mathrm{TiO}_{2}$, mechanism, adsorption.

123. Fujishima, A., R. Cai, K. Hashimoto, H. Sakai, and Y. Kubota. "Biochemical Application of Titanium Dioxide Photocatalysts." Photocatalytic Purification and Treatment of Water and Air, eds. David F. Ollis and Hussain Al-Ekabi, 193-205, Trace Metals and the Environment' ed. Jerome 0. Nriagu, New York, NY: Elsevier, 1994.

$\mathrm{TiO}_{2}$, disinfection, aqueous phase, application.

124. Fujishima, Akira "Applications of Semiconductor Photocatalysts." Kikan Kagaku Sosetsu 23 (1994): 129-38.

$\mathrm{TiO}_{2}$, disinfection, application.

125. Fujishima, Akira "Highly Effective Photocatalytic Reactions using $\mathrm{TiO}_{2}$ Surface." Presented at 17th U.S.-Japan Cooperative Seminar on Photoconversion and Photosynthesis Research, La Jolla, CA, 12-14 March 1995, 3 pp.

126. Fujishima, Akira, Ru Xiong Cai, Hideki Sakai, Ryo Baba, Kazuhito Hashimoto, and Yoshinobu Kubota. "Detection of Intermediates Formed At the Surface of Photo-Excited Titanium Dioxide and Biological Application of Titanium Dioxide Photocatalysts." Proc.-Electrochem. Soc. 93-1 1, no. Proceedings of the Fifth International Symposium on Redox Mechanisms and Interfaces (1993): 363-72.

$\mathrm{TiO}_{2}$, disinfection, aqueous phase, mechanism, application.

127. Fujita, Jinji, Masahiro Kubo, Emi Fumrukawa, Makiko Higashikawa, and Keizou Nakano. "Photocatalytic Decomposition of Chlorpyrifos in the Presence of Titanium Dioxide." Kugawa-Ken Kankyo Kenkyu Senta Shoho 18 (1994): 31-4.

$\mathrm{TiO}_{2}$, pesticide, mechanism.

128. Fukui, Hiroshi, Yukiko Hashimoto, Michihiro Yamaguchi, and Shoichi Anho, inventors. "Magnetic Photocatalysts." Japan Shiseido Co Ltd, assignee. Japan Patent, 06154620 A2.3 June 1994. CA121:219967.

$\mathrm{TiO}_{2}$, other semiconductor, engineering, modified $\mathrm{TiO}_{2}$, application.

129. Funayama, Hitoshi, and Takuo Sugawara "Photocatalytic Decomposition Rate of Chloroform in Dilute Aqueous Solution in a Titanium Dioxide Suspension." Kagaku Kogaku Ronbunshu 19, no. 2 (1993): 272-8.

$\mathrm{TiO}_{2}$, aqueous phase. 
130. Galvez, Julian Blanco, and Sixto Malato Rodriguez. "Influence of Solar Irradiation Over Pentachlorophenol Solar Photocatalytic Decomposition." Photocutulytic Purification and Treatment of Water and Air, eds. David F. Ollis and Hussain Al-Ekabi, 63944, Trace Metals and the Environment, ed. Jerome 0. Nriagu, New York, NY: Elsevier, 1994.

$\mathrm{TiO}_{2}$, oxidant, aqueous phase, process efficiency.

131. Gao, Cuiqin, Yulin Dong, and Qinhua Dong. "Study on the Decomposition of Organophosphorous Insecticide in Water by Using Solar Energy." Wuhan Daxue Xuebao, Ziran Kexuebun, no. 3 (1992): 73-7.

$\mathrm{TiO}_{2}$, solar, pesticide, aqueous phase.

132. Gao, Cuiqin, Jianzhen Shi, Qinghua Dong, and Yu Guo. "Photocatalytic Decomposition of Humic Acid in Water in the Presence of Semiconductor Suspension." Huaxue Shijie 34, no. 8 (1993): 390-3.

$\mathrm{TiO}_{2}$, solar, aqueous phase.

133. Gerischer, H. "Conditions for an Efficient Photocatalytic Activity of Titanium Dioxide Particles." Photocatalytic Purification and Treatment of Water and Air, eds. David F. Ollis and Hussain Al-Ekabi, 1-17, Trace Metals and the Environment, ed. Jerome 0. Nriagu, New York, NY: Elsevier, 1994. $\mathrm{TiO}_{2}$, modeling, aqueous phase, process efficiency, mechanism.

134. Gimeniz-Farreras, Jaime, M. A. Aguado, S. Cervera, L. Borrell, D. Curco, and M. A. Queral. "Photoreactor Design for Photocatalytical Detoxification: Kinetic and Radiation Studies. " Solar Engineering 1994, eds. David E. Klett, Roy E. Hogan, and Tadayoshi Tanaka, 139-45, 1994.

$\mathrm{TiO}_{2}$, engineering, reactor, modeling, solar, oxidant, aqueous phase, metal removal.

135. Goldberg, Marvin C., Kirkwood M. Cunningham, and Eugene R. Weiner. "Aquatic Photolysis: Photolytic Redox Reactions Between Geothite and Adsorbed Organic Acids in Aqueous Solutions." J. Photochem. Photobiol. A: Chem. 73 (1993): 105-20.

other semiconductor, solar, reductant, aqueous phase, mechanism.

136. Goldstein, Sara' Gidon Czapski, and Joseph Rabani. "Oxidation of Phenol by Radiolytically Generated $\mathrm{OH}$ Radical and Chemically Generated $\mathrm{SO}_{4}(-1)$ Radical. A Distinction Between $\mathrm{OH}$ Transfer and Hole Oxidation in the Photolysis of $\mathrm{TiO}_{2}$ Colloid Solution." J. Phys. Chem. 98, no. 26 (1994): 6586-9 1.

$\mathrm{TiO}_{2}$, oxidant, aqueous phase, mechanism, metal removal.

137. Goswami, D. Y., J. Klausner, G. D. Mathur, A. Martin, K. Schanze, and P. Wyness. Solar Photocatalytic Treatment of Groundwater At Tyndall AFB: Field Test Results, University of Florida, Gainesville, FL, 1993.

engineering, aqueous phase, oxidant, solar, $\mathrm{TiO}_{2}$.

138. Goswami, D. Yogi, J. Klausner, G. D. Mathur, A. Martin, K. Schanze, P. Wyness, Craig T. Turchi, and E. Marchand. "Solar Photocatalytic Treatment of Groundwater At Tyndall AFB: Field Test Results." Solarl 993. Proceedings of the American Solar Energy Society Annual Conference, 235-9, Boulder, CO: ASES, 1993.

aqueous phase, engineering, process efficiency, oxidant' adsorbent, $\mathrm{TiO}_{2}$. 
139. Goswami, D. Yogi, Dhara M. Trivedi, and Seymour S. Block "Photocatalytic Disinfection of Indoor Air." Solar Engineering 1995, eds. William B. Stine, Tadayoshi Tanaka' and David E. Claridge, 421-7, New York, NY: ASME, 1995.

$\mathrm{TiO}_{2}$, reactor, disinfection, gas phase, application, IAQ, immobilized $\mathrm{TiO}_{2}$.

140. Gray, K. A., U. Stafford, M. S. Dieckmann, and P. Kamat. "Mechanistic Studies in Titanium Dioxide Systems: Photocatalytic Degradation of Chloro- and Nitrophenols." Photocatalytic Purification and Treatment of Water and Air, eds. David F. Ollis and Hussain Al-Ekabi, 455-72, Trace Metals and the Environment' ed. Jerome 0. Nriagu, New York, NY: Elsevier, 1994. $\mathrm{TiO}_{2}$, aqueous phase, mechanism, immobilized $\mathrm{TiO}_{2}$, adsorption.

141. Gray, Kimberly A., and Ulick Stafford. "Probing Photocatalytic Reactions in Semiconductor Systems: Study of the Chemical Intermediates in 4-Chlorophenol Degradation by a Variety of Methods." Res. Chem. Intermed. 20, no. 8 (1994): 835-53.

$\mathrm{TiO}_{2}$, aqueous phase, mechanism.

142. Gray, Kimberly A., and Ulick Stafford. "Probing Photocatalytic Reactions in Semiconductor Systems: Study of the Chemical Intermediates in 4-Chlorophenol Degredation by a Variety of Methods." Res. Chem Intermed. 20, no. 8 (1994): 835-53.

$\mathrm{TiO}_{2}$, aqueous phase, modified $\mathrm{TiO}_{2}$, mechanism, immobilized $\mathrm{TiO}_{2}$, adsorbent.

143. Green, Kevin J., and Robert Rudham. "Photocatalytic Oxidation of Propan-2-ol by Semiconductor-Zeolite Composites." J. Chem. Soc., Faraday Trans. 89, no. 11 (1993): 1867-70. nonaqueous, $\mathrm{TiO}_{2}$, other semiconductor, modified $\mathrm{TiO}_{2}$, mechanism, immobilized $\mathrm{TiO}_{2}$.

144. Hagfeldt, Anders, Henrik Lindstrom, Sven Sodergren, and Sten-Eric Lindquist. "Photoelectochemical Studies of Colloidal $\mathrm{TiO}_{2}$ Films: The Effect of Oxygen Studied by Photocurrent Transients." J. Electroanalytical Chem. 381 (1995): 39-46.

$\mathrm{TiO}_{2}$, oxidant, aqueous phase, process efficiency, mechanism, immobilized $\mathrm{TiO}_{2}$.

145. Hamid, S. H., M. B. Amin, A. G. Maadhah, and A. M. Al-Jarallah. "Polymer Lifetime Studies in Hostile Environments." J. Vinyl Technol. 14, no. 4 (1992): 182-6.

plastics, $\mathrm{TiO}_{2}$, solar, application, mechanism.

146. Harada, Hisashi. "Photocatalysis by Semiconductors Loaded With Metal." Kikan Kagaku Sosetsu 23 (1994): 69-77.

$\mathrm{TiO}_{2}$, metallized $\mathrm{TiO}_{2}$, aqueous phase, modified $\mathrm{TiO}_{2}$, mechanism.

147. Harada, Hisashi. "Photocatalytic Reactions for Aliphatic Dicarboxylic Acids Using MetalLoaded Titanium Oxide.” Res. Bull. Meisei Univ., Phys. Sci Eng. 29 (1993): 27-36. $\mathrm{TiO}_{2}$, metallized $\mathrm{TiO}_{2}$, aqueous phase, nonaqueous, mechanism.

148. Hasegawa, Kiyoshi, Masanori Murase, Masao Kuboshita, Hidetaka Saida, Misao Shinoda, Masatoshi Miyamoto, Choichiro Shimasaki, Toshiaki Yoshimura, Eiichi Tsukurimichi, and Shigeya Takeuchi. "Photooxidation of Naphthalenamines Adsorbed on Particles Under Simulated Atmospheric Conditions." Environ. Sci. Technol. 27, no. 9 (1993): 1819-25.

$\mathrm{TiO}_{2}$, other semi:onductor, reactor, solar, gas phase. 
149. Hasegawa, Shigeo, Hiroyuki Ozora, Kazuto Kobayashi, and Yoshimasa Fujimoto, inventors. "Photocatalyst Composite." Ltd Mitsubishi Heavy Industries, assignee. Japan Patent, 04334552 A2. 20 November 1992. CA1 18(14): 133096n.

$\mathrm{TiO}_{2}$, other semiconductor, modified $\mathrm{TiO}_{2}$, immobilized $\mathrm{TiO}_{2}$.

150. Hashimoto, Kazuhito, and Akira Fujishima. "Removal of Environmental Pollutants Using Photocatalytic Reactions." Kagaku Sochi 36, no. 4 (1994): 77-8 1.

$\mathrm{TiO}_{2}$, gas phase, aqueous phase, application, immobilized $\mathrm{TiO}_{2}$.

151. Heleg, Vered, and Itamar Willner. "Photocatalyzed CO,-Fixation to Formate and $\mathrm{H}_{2}$ Evolution by Eosin-Modified Pd-TiO 2 Powders." J. Chem. Soc., Chem. Commun. (1994): 2113-4. $\mathrm{TiO}_{2}$, solar, reductant, metallized $\mathrm{TiO}_{2}$, aqueous phase, modified $\mathrm{TiO}_{2}$.

152. Heller, Adam, and James R. Brock, inventors. "Materials and Methods for Enhanced Photocatalysis of Organic Compounds in Oil Spill Treatment." University of Texas System, assignee. World Patent, 9317971 Al. 16 September 1993. CA120(2):14565w.

$\mathrm{TiO}_{2}$, other semiconductor, engineering, solar, gas phase, aqueous phase, application, immobilized $\mathrm{TiO}_{2}$, adsorbent, biotreatment.

153. Heller, Adam, Maya Nair, Lois Davidson, Zhenghao Luo, Jorg Schwitzgebel, Jeffery Norrell, James R. Brock, Sten Eric Lindquist, and John G. Ekerdt. "Photoassisted Oxidation of Oil and Organic Spills on Water." Photocatalytic Purification and Treatment of Water and Air, eds. David F. Ollis and Hussain Al-Ekabi, 139-53, Trace Metals and the Environment, ed. Jerome 0. Nriagu, New York, NY: Elsevier, 1994.

$\mathrm{TiO}_{2}$, solar, aqueous phase, modified $\mathrm{TiO}_{2}$, application, process efficiency, immobilized $\mathrm{TiO}_{2}$, adsorbent.

154. Heller, Adam, Joerg Schwitzgebel, Michael V. Pishko, and J. G. Ekerdt. "Environmental Photoelectrochemistry." Proc. - Electrochem. Soc. 94-19 (Water Purification by Photocatalytic Photoelectrochemical, and Electrochemical Processes) (1994): 1-9. $\mathrm{TiO}_{2}$, oxidant, aqueous phase, modified $\mathrm{TiO}_{2}$, process efficiency, mechanism, immobilized $\mathrm{TiO}_{2}$.

155. Herrmann, Jean-Marie, Jean Disdier, and Pierre Pichat. "Photocatalytic Deposition of Silver on Powder Titania: Consequences for the Recovery of Silver." J.Catal. 113 (1988): 72-81. $\mathrm{TiO}_{2}$, oxidant, metallized $\mathrm{TiO}_{2}$, aqueous phase, application, process efficiency, metal removal.

156. Hidaka, H., J. Zhao, K. Nohara, K. Kitamura, Y. Satoh, E. Pelizzetti, and N. Serpone. "Photocatalyzed Mineralization of Non-Ionic, Cationic, and Anionic Surfactants At Titanium Dioxide,Water Interfaces." Photocatalytic Purification and Treatment of Water and Air, eds. David F. Ollis and Hussain Al-Ekabi, 251-9, Trace Metals and the Environment' ed. Jerome 0. Nriagu, New York, NY: Elsevier, 1994.

$\mathrm{TiO}_{2}$, aqueous phase.

157. Hidaka, Hisao, Kayo Nohara, Kazuhiko Ooishi, Jincai Zhao, Nick Serpone, and Ezio Pelizzetti. "Photodegradation of Surfactants. XV: Formation of SO42- Ions in the Photooxidation of Sulfur-Containing Surfactants." Chemosphere 29, no. 12 (1994): 26194. $\mathrm{TiO}_{2}$, aqueous phase, other semiconductor, application. 
158. Hidaka, Hisao, Kayo Nohara, Jincai Zhao, Keiko Takashima, Ezio Pelizzetti, and Nick Serpone. "Photodegradation of Surfactants. XIII. Photocatalytic Mineralization of Nitrogen-Containing Surfactants At the $\mathrm{TiO}_{2}$,Water Interface." New J. Chem. 18, no. 4 (1994): 541-5.

$\mathrm{TiO}_{2}$, aqueous phase.

159. Hidaka, Hisao, and Jincai Zhao. "Photodegradation of Surfactants Catalyzed by a Titania Semiconductor." Colloids Surf. 67 (1992): 165-82.

$\mathrm{TiO}_{2}$, aqueous phase.

160. Hidaka, Hisao, Jincai Zhao, Satoshi Horikoshi, Nick Serpone, and Ezio Pelizzetti. "The Photodegradation of Surfactants. XVI. Photocatalytic Effect of Various Semiconductors on the Photooxidation of Surfactants." Yukagaku 44, no. 2 (1995): 121-5.

$\mathrm{TiO}_{2}$, other semiconductor, metallized $\mathrm{TiO}_{2}$, aqueous phase, modified $\mathrm{TiO}_{2}$.

161. Hidaka, Hisao, Jincai Zhao, and Kayo Nohara. "Photodegradation of Surfactants Using Titanium Dioxide." Yosui to Haisui 36, no. 10 (1994): 863-70.

$\mathrm{TiO}_{2}$, aqueous phase, pesticide.

162. Hidaka, Hisao, Jincai Zhao, Yasuhito Satoh, Kayo Nohara, Ezio Pelizzetti, and Nick Serpone. "Photodegradation of Surfactants. Part XII: Photocatalyzed Mineralization of Phosphorus-Containing Surfactants At $\mathrm{TiO}_{2}, \mathrm{H}_{2} \mathrm{O}$ Interfaces." J. Mol. Catal. 88, no. 2 (1994):239-48. $\mathrm{TiO}_{2}$, aqueous phase.

163. Higashi, Kunihiko, and Kenji Otsuka. "Treatment of Hypophosphite lon in Waste Electroless Nickel Plating Solution by Photocatalysis.” Kogyo Yosui 415 (1993): 34-9. $\mathrm{TiO}_{2}$, reductant, aqueous phase, application, metal removal.

164. Hilgendorff, M., M. Hilgendorff, and D. W. Bahnemann. "Photocatalytic Reduction of Perhalogenated Hydrocarbons on Platinized Titanium Dioxide in Aqueous Solution." J. Inf. Rec. Mater. 21, no. 5-6 (1994): 697-8.

$\mathrm{TiO}_{2}$, metallized $\mathrm{TiO}_{2}$, aqueous phase, reductant.

165. Hirano, Katsuhiko, Kazuhisa Funaki, and Akio Hoshino. "Electron Transfer to Metal Particles From Photoilluminated N-Titania Electrode. Detection of the Electron-Loaded Particles by RRDE." Denki Kagaku Oyobi Kogyo Butsuri Kagaku 61, no. 4 (1993): 446-7.

$\mathrm{TiO}_{2}$, reductant, metallized $\mathrm{TiO}_{2}$, aqueous phase, process efficiency, mechanism, metal removal.

166. Hirano, Katsuhiko, Kohei Inoue, and Tomomi Yatsu. "Photocatalyzed Reduction of CO, in Aqueous $\mathrm{TiO}_{2}$ Suspension Mixed With Copper Powder." J. Photo\&em. and Photobiol. A: Chem. 64 (1992): 255-8.

$\mathrm{TiO}_{2}$, reductant, aqueous phase.

167. Hisanaga, Teruaki, and Keiichi Tanaka. "Effect of the Crystal Type of Titanium Dioxide on Photocatalytic Degradation of Organohalogen Compounds." Mizu Shori Gijutsu 34, no. 1 (1993): 13-18.

$\mathrm{TiO}_{2}$, aqueous phase, modified $\mathrm{TiO}_{2}$, mechanism.

168. Hisanaga, Teruaki, and Keiichi Tanaka, inventors. "Manufacture of Fixed Photocatalysts." Kogyo Gijutsuin, assignee. Japan Patent, 05096180 A2. 20 April 1993. CA119(10):102684u. $\mathrm{TiO}_{2}$, other semiconductor, aqueous phase, modified $\mathrm{TiO}_{2}$, application, immobilized $\mathrm{TiO}_{2}$. 
169. Hisanaga, Teruaki, and Keiichi Tanaka. "Photocatalytic Degradation of Harmful Compounds in Gas Phase by the Illumination With Short Wavelength UV." Denki Kagaku Oyobi Kogyo Butsuri Kagaku 63, no. 3 (1995): 212-16.

$\mathrm{TiO}_{2}$, gas phase.

170. Hoffman, A. J. "Photocatalytic Reactions on Quantum-Sized Semiconductor Colloids: Photoinitiated Polymerization of Vinylic Monomers, Formation of Hydrogen Peroxide and Organic Peroxides, Oxidation of Carboxylic Acids, and Synthesis of Humic-Like Material." Ph.D. diss., California Institute of Technology, 1993.

aqueous phase, modified $\mathrm{TiO}_{2}$, other semiconductor, oxidant.

171. Hoffmann, Michael R., Scot T. Martin, Wonyong Choi, and Detlef W. Bahnemann. "Environmental Applications of Semiconductor Photocatalysis." Chem. Rev. 95, no. 1 (1995): 69-96. $\mathrm{TiO}_{2}$, other semiconductor, engineering, reactor, modeling, metallized $\mathrm{TiO}_{2}$, gas phase, aqueous phase, modified $\mathrm{TiO}_{2}$, application, process efficiency, mechanism, metal removal.

172. Hofstadler, K., Rupert Bauer, S. Novalic, and G. Heisler. "New Reactor Design for Photocatalytic Wastewater Treatment With $\mathrm{TiO}_{2}$ Immobilized on Fused-Silica Glass Fibers: Photomineralization of 4-Chlorophenol." Environ. Sci. Technol. 28, no. 4 (1994): 670-4. $\mathrm{TiO}_{2}$, engineering, aqueous phase, modified $\mathrm{TiO}_{2}$, application, immobilized $\mathrm{TiO}_{2}$.

173. Hofstadler, K., G. Ruppert, R. Bauer, G. Heisler, and S. Novalic. "Photocatalyzed Treatment of 4-Chlorophenol With Immobilized Titanium Dioxide." Photocatalytic Purification and Treatment of Water and Air, eds. David F. Ollis and Hussain Al-Ekabi, 777-82, Trace Metals and the Environment' ed. Jerome 0. Nriagu, New York, NY: Elsevier, 1994.

$\mathrm{TiO}_{2}$, oxidant, aqueous phase, immobilized $\mathrm{TiO}_{2}$.

174. Holden, Wendy, Angelica Marcellino, Damir Valic, and Alan C. Weedon. "Titanium Dioxide Mediated Photochemical Destruction of Trichloroethylene Vapors in Air. "Photocatalytic Purification and Treatment of Water and Air, eds. David F. Ollis and Hussain Al-Ekabi, 393404, Trace Metals and the Environment' ed. Jerome 0. Nriagu, New York, NY: Elsevier, 1994.

$\mathrm{TiO}_{2}$, gas phase, immobilized $\mathrm{TiO}_{2}$.

175. Hsieh, Yung-Hsu, Kuo-Shu Huang, and Kuo-Hua Wang. "Study on the Photocatalytic Degradation of Monochlorophenol Pollutants by Titanium Dioxide in Aqueous Solutions."

Organohalogen Compd. 19, Dioxin '94 (1994): 503-7.

$\mathrm{TiO}_{2}$, aqueous phase, application, mechanism.

176. Hsieh, Yung Hsu, and Kuo Hua Wang. "Photocatalytic Decomposition of 2-Chlorophenol Over Titanium Dioxide." Xingda Gongcheng Xuebao 4 (1993): 85-93. $\mathrm{TiO}_{2}$, aqueous phase, mechanism.

177. Hung, Sze To, and Mark K. S. Mak. "Titanium Dioxide Photocatalyzed Degradation of Organophosphate in a System Simulating the Natural Aquatic Environment" Environ. Technol. 14, no. 3 (1993): 265-9.

aqueous phase, application, photocatalysis, $\mathrm{TiO}_{2}$, Malathion, DDVP, fulvic acid, pesticide.

178. Hwang, Bing Joe, and Shyh Song Jeng. "Photoelectrochemical Study for Destruction of Cyanide Waste With Modified $\mathrm{TiO}_{2}$ Electrodes." J. Chin. Inst. Chem. Eng. 24, no. 6 (1993): 401-6. $\mathrm{TiO}_{2}$, metallized $\mathrm{TiO}_{2}$, aqueous phase, modified $\mathrm{TiO}_{2}$, application, process efficiency, immobilized $\mathrm{TiO}_{2}$. 
179. Ibusuki, Takashi. "Nitrogen Oxides Reducing Technology by Photocatalysts." Kogyo Zairyo 41, no. 13 (1993): 59-64.

$\mathrm{TiO}_{2}$, gas phase, modified $\mathrm{TiO}_{2}$, application, immobilized $\mathrm{TiO}_{2}$, adsorbent.

180. Ibusuki, Takashi. "Nitrogen Oxides Removal by Photocatalytic Oxidation." PPM 24, no. 10 (1993): 66-72.

$\mathrm{TiO}_{2}$, solar, engineering, gas phase, aqueous phase, modified $\mathrm{TiO}_{2}$, application, immobilized $\mathrm{TiO}_{2}$, catalyst reactivation.

181. Ibusuki, Takashi, and Takeuchi Koji. "Photocatalytic Effect of Titanium Dioxide in Trans-2- $\mathrm{C}_{4} \mathrm{H}_{8} / \mathrm{O}_{2}$ and Trans-2- $\mathrm{C}_{4} \mathrm{H}_{8} / \mathrm{NO}_{2}$, Air Reaction Systems." Tatki Osen Gakkaishi 20, no. 2 (1985): $82-8$.

$\mathrm{TiO}_{2}$, reactor, oxidant, gas phase.

182. Ibusuki, Takashi, Shuzo Kutsuna, Koji Takeuchi, Kazuteru Shinkai, Toshiharu Sasamoto, and Masahiro Miyamoto. "Removal of Low Concentration Air Pollutants Through Photoassisted Heterogeneous Catalysis. " Photocatalytic Purification and Treatment of Water and Air, eds.

David F. Ollis and Hussain Al-Ekabi, 375-86, Trace Metals and the Environment' ed. Jerome 0. Nriagu, New York, NY: Elsevier, 1994.

$\mathrm{TiO}_{2}$, gas phase, modified $\mathrm{TiO}_{2}$, application, immobilized $\mathrm{TiO}_{2}$, adsorbent, catalyst reactivation.

183. Ibusuki, Takashi, Keigo Nakamura, and Schuzou Kutsuna, inventors. "Method for Photochemical Decomposition of Volatile Organic Chlorine Compound in Vent Gas." Agency of Industrial Science and Technology, assignee. Japan Patent, 4966665.30 October 1990.

gas phase, immobilized $\mathrm{TiO}_{2}, \mathrm{TiO}_{2}$.

184. Ibusuki, Takashi, and Koji Takeuchi. "Removal of Low Concentration Nitrogen Oxides Through Photoassisted Heterogeneous Catalysis." J. Mol. Catal. 88, no. 1 (1994): 93-102.

$\mathrm{TiO}_{2}$, engineering, gas phase, modified $\mathrm{TiO}_{2}$, application, immobilized $\mathrm{TiO}_{2}$, adsorbent, catalyst reactivation.

185. lbusuki, Takashi, Koji Takeuchi, Kazuteru Shinkai, Satoshi Nishikata, Masahiro Miyamoto, Yukihiro Noguchi, and Takeo Takahashi, inventors. "Photocatalyst for Removing Pollutants From Air." Ltd. Japan Fuji Electric Co., and Agency of Industrial Sciences and Technology, assignees. Europe Patent, 614682 Al. 14 September 1994. CA121:237630.

$\mathrm{TiO}_{2}$, engineering, reactor, solar, gas phase, modified $\mathrm{TiO}_{2}$, application, immobilized $\mathrm{TiO}_{2}$, adsorbent, catalyst reactivation.

186. Inoue, Hiroshi, Takehiko Matsuyama, Bi Jin Liu, Takao Sakata, Hirotaro Mori, and Hiroshi Yoneyama "Photocatalytic Activities of $\mathrm{TiO}_{2}$ Microcrystals Prepared in Si02 Matrixes Using a Sol-Gel Method for Carbon Dioxide Reduction." Chem. Lett., no. 3 (1994): 653-6. $\mathrm{TiO}_{2}$, reductant, gas phase, aqueous phase, modified $\mathrm{TiO}_{2}$, immobilized $\mathrm{TiO}_{2}$.

187. Ion, Rodica Mariana, and Viorel Gazdaru. "New Efficient Metalloporphyrin Photocatalysts for Kraft Lignin Photodegradation.” Prog. Catal. 1, no. 2 (1992): 21-7.

Lignin, $\mathrm{TiO}_{2}$, aqueous phase, modified $\mathrm{TiO}_{2}$, application. 
188. Ireland, John C., Brunilda Davila, Hector Moreno, Shannon K. Fink, and Stephanie Tassos. "Heterogeneous Photocatalytic Decomposition of Polyaromatic Hydrocarbons Over Titanium Dioxide." Chemosphere 30, no. 5 (1995): 965-84.

$\mathrm{TiO}_{2}$, aqueous phase, application.

189. Ireland, John C., Petra Klostermann, Eugene W. Rice, and Robert M. Clark "Inactivation of Escherichia Coli by Titanium Dioxide Photocatalytic Oxidation." Appl. Environ. Microbiol.59, no. 5 (1993): 1668-70.

$\mathrm{TiO}_{2}$, disinfection, aqueous phase.

190. Irick, Gether Jr, inventor. "Photoactive Catalyst of Barium Phosphate or Calcium Phosphate Supported on Anatase Titanium Dioxide." Eastman Kodak Co, assignee. United States Patent, 5242880 A. 7 September 1993. CA120(6):55977a.

Plastics Manufacture and Processing, $\mathrm{TiO}_{2}$, aqueous phase, modified $\mathrm{TiO}_{2}$, application.

191. Ishitani, Osamu, Chieko Inoue, Yuji Suzuki, and Takashi Ibusuki. "Photocatalytic Reduction of Carbon Dioxide to Methane and Acetic Acid by an Aqueous Suspension of Metal-Deposited Titania." J. Photochem. Photobiol., A 72, no. 3 (1993): 269-7 1.

$\mathrm{TiO}_{2}$, reductant, metallized $\mathrm{TiO}_{2}$, aqueous phase.

192. Isidorov, V. A. "Non-Methane Hydrocarbons in the Atmosphere of Boreal Forests: Composition, Emission Rates, Estimation of Regional Emission and Photocatalytic Transformation." Ecol. Bull. 42, Trace Gas Exchange in a Global Perspective (1992): 71-76.

other semiconductor, modeling, solar, gas phase.

193. Ito, Takehiko, inventor. "Iron Oxide Photocatalyst." Japan Mitsubishi Heavy Ind Ltd, assignee. Japan Patent' 06039285 A2.15 February 1994. CA121:46470.

$\mathrm{TiO}_{2}$, other semiconductor, aqueous phase, modified $\mathrm{TiO}_{2}$, application.

194. Jackson, N. B., C. M. Wang, Z. Luo, J. Schwitzgebel, J. Norrell, J. R. Brock, and A. Heller. "Attachment of $\mathrm{TiO}_{2}$ Powders to Hollow Glass Microbeads: Activity of the $\mathrm{TiO}_{2}$ Coated Beads to the Photoassisted Oxidation of Ethanol to Acetaldehyde." J. Electrochem. Soc. 138 (199 1): 3660-4. $\mathrm{TiO}_{2}$, aqueous phase, immobilization $\mathrm{TiO}_{2}$.

195. Jacoby, William A., Daniel M. Blake, LeAnn M. Vargo, John A. Fennell, Marya C. George, Suzanne K. Dolberg, and James E. Boulter. "Heterogeneous Photocatalysis for Control of Volatile Organic Compounds and Bioaerosols in Indoor Air." Presented at Engineering Solutions to Indoor Air Quality Problems, Research Triangle Park, NC, 24-26 July 1995, 28 pp.

196. Jacoby, William A., Mark R. Nimlos, Daniel M. Blake, Richard D. Noble, and Carl A. Koval. "Products, Intermediates, Mass Balances, and Reaction Pathways for the Oxidation of Trichloroethylene in Air Via Heterogeneous Photocatalysis." Environ. Sci. Technol. 28, no. 9 (1994): 1661-68. $\mathrm{TiO}_{2}$, gas phase, process efficiency, mechanism, immobilized $\mathrm{TiO}_{2}$.

197. Jakob, Laurent, Esther Oliveros, Omar Legrini, and Andre M. Braun. "Titanium Dioxide Photocatalytic Treatment of Water. Reactor Design and Optimization Experiments. "Photocatalytic Purification and Treatment of Water and Air, eds. David F. Ollis and Hussain Al-Ekabi, 5 1 1-32, Trace Metals and the Environment' ed. Jerome 0. Nriagu, New York, NY: Elsevier, 1994. $\mathrm{TiO}_{2}$, reactor, modeling, aqueous phase, application, engineering. 
198. Jardim, Wilson F., Rosana M. Alberici, Marcia M. K. Takiyama, and C. P. Huang. "Gas-Phase Photocatalytic Destruction of Trichloroethylene (TCE) Using UV,TiO ${ }_{2}$ " Hazard. Ind. Wastes 26 (1994): 230-8.

$\mathrm{TiO}_{2}$, engineering, reactor, gas phase, immobilized $\mathrm{TiO}_{2}$.

199. Johnston, A. J., and P. Hocking. "Ultrasonically Accelerated Photocatalytic Waste Treatment." ACS Symp. Ser. 518, Emerging Technologies in Hazardous Waste Management III (1993): 106-8.

$\mathrm{TiO}_{2}$, engineering, reactor, aqueous phase, application, process efficiency, sonication.

200. Joselevich, Emesto, and Itamar Willner. "Photosensitization of Quantum-Sized $\mathrm{TiO}_{2}$ Particles in Water-in-Oil Microemulsions." J. Phys. Chem. 98, no. 31 (1994): 7628-35.

$\mathrm{TiO}_{2}$, aqueous phase, microemulsion.

201. Judin, Vesa P. S. "The Lighter Side of TiO $_{2}$." Chem. Brit. June (1993): 503-5. $\mathrm{TiO}_{2}$, modified $\mathrm{TiO}_{2}$.

202. Kadowaki, Satoru, Makoto Suzuki, Kunio Okamoto, and Yoshimasa Kodama, inventors. "Deodorizing Agents." Japan Nippon Denso Co, and Nippon Soken, assignees. Japan Patent' 07024256 A2.27 January 1995. CA122:247162.

$\mathrm{TiO}_{2}$, other semiconductor, engineering, gas phase, application, immobilized $\mathrm{TiO}_{2}$, adsorbent.

203. Kaise, Masahiro, Hidekazu Nagai, Kazuaki Tokuhashi, Shigeo Kondo, Shigeaki Nimura, and Osamu Kikuchi. "Electron Spin Resonance Studies of Photocatalytic Interface Reactions of Suspended $\mathbf{M}_{\mathbf{T i O}}(\mathrm{M}=\mathrm{Pt}, \mathrm{Pd}, \mathrm{Ir}, \mathrm{Rh}, \mathrm{Os}$, or Ru) With Alcohol and Acetic Acid in Aqueous Media" Langmuir 10, no. 5 (1994): 1345-7.

$\mathrm{TiO}_{2}$, metallized $\mathrm{TiO}_{2}$, mechanism.

204. Kamat, Prashant K. "Nanocrystalline Semiconductor Thin Films for Microelectronic and Optoelectronic Applications." Mat. Tech. 9 (1994): 147-9.

$\mathrm{TiO}_{2}$, other semiconductor, application.

205. Kamat, Prashant V. "Interfacial Charge Transfer Processes in Colloidal Semiconductor Systems." Prog. Reaction Kinetics 19 (1994): 277-3 16.

$\mathrm{TiO}_{2}$, other semiconductor, modeling, oxidant, reductant, aqueous phase, modified $\mathrm{TiO}_{2}$, mechanism.

206. Kamat, Prashant V. "What Makes Semiconductor Colloids Unique As Photocatalysts?" The Spectrum 6 (1993): 14-20.

$\mathrm{TiO}_{2}$, other semiconductor, aqueous phase, mechanism.

207. Kamat, Prashant V., Idris Bedja, and Surat Hotchandani. "Photoinduced Charge Transfer Between Carbon and Semiconductor Clusters. One-Electron Reduction of $\mathrm{C}_{6} \mathrm{O}$ in Colloidal $\mathrm{TiO}_{2}$

Semiconductor Suspensions." J. Phys. Chem. 98, no. 37 (1994): 913742.

$\mathrm{TiO}_{2}$, nonaqueous, modified $\mathrm{TiO}_{2}$, process efficiency, application.

208. Kamat, Prashant V., Idriss Bedja, and Surat Hotchandani. "Photoinduced Charge Transfer Between Fullerenes and $\mathrm{TiO}_{2}$ Semiconductor Colloids." Proc.-Electrochem. Soc.94-24(Recent Advances in the Chemistry and Physics of Fullerenes and Related Materials) (1994): 964-75.

nonaqueous, $\mathrm{TiO}_{2}$. 
209. Kamat, Prashant V., and K. Vinodgopal. "Titanium Dioxide Mediated Photocatalysis Using Visible Light: Photosensitization Approach. " Photocatalytic Purification and Treatment of Water and Air, eds. David F. Ollis and Hussain Al-Ekabi, 83-94, Trace Metals and the Environment, ed. Jerome 0. Nriagu, New York, NY: Elsevier, 1994.

$\mathrm{TiO}_{2}$, modeling, aqueous phase, modified $\mathrm{TiO}_{2}$, application.

210. Kato, K., A. Tsuzuki, H. Taoda, Y. Torii, T. Kato, and Y. Butsugan. "Crystal Structures of $\mathrm{TiO}_{2}$ Thin Coatings Prepared From the Alkoxide Solution Via the Dip-Coating Technique Affecting the Photocatalytic Decomposition of Aqueous Acetic Acid.” J. Mater. Sci 29, no. 22 (1994): 591 1-15. $\mathrm{TiO}_{2}$, aqueous phase, modified $\mathrm{TiO}_{2}$, immobilized $\mathrm{TiO}_{2}$.

211. Kato, Kazumi. "Synthesis of Titanium Dioxide Photocatalysts With High Activity by the Alkoxide Method." Photocatalytic Purification and Treatment of Water and Air, eds. David F. Ollis and Hussain Al-Ekabi, 809-13, Trace Metals and the Environment, ed. Jerome 0. Nriagu, New York, NY: Elsevier, 1994.

$\mathrm{TiO}_{2}$, aqueous phase, application, modified $\mathrm{TiO}_{2}$.

212. Kato, Kazumi, Akihiro Tsuzuki, Yasuyoshi Torii, Hiroshi Taoda, Tamihiko Kato, and Yasuo Butsugan. "Preparation of Titanium Dioxide Thin Coatings As Photocatalyst With High Activity by Sol-Gel Method." Nagoya Kogyo Gijutsu Kenkyusho Hokoku 42, no. 12 (1994): 346-53. $\mathrm{TiO}_{2}$, aqueous phase, modified $\mathrm{TiO}_{2}$.

213. Kato, Tamihiko, Yasuo Butsugan, Kazumi Kato, Boon Hian Loo, and Akira Fuishima. "Decomposition of Aqueous Poly(Vinyl Alcohol) on Photoexcited Titanium Dioxide." Denki Kagaku Oyobi Kogyo Butsuri Kagaku 61, no. 7 (1993): 876-7.

Polymers, $\mathrm{TiO}_{2}$, aqueous phase, application.

214. Kawaguchi, Hideki. "Dependence of Photocatalytic Reaction Rate on Titanium Dioxide Concentration in Aqueous Suspensions.” Environ. Technol. 15, no. 2 (1994): 183-8.

$\mathrm{TiO}_{2}$, modeling, aqueous phase.

215. Kawaguchi, Hideki. "Dependence of Photocatalytic Reaction Rate on Photocatalyst Concentration in Aqueous Suspensions." Photocatalytic Purification and Treatment of Water and Air, eds. David F. Ollis and Hussain Al-Ekabi, 665-82, Trace Metals and the Environment' ed. Jerome 0. Nriagu, New York, NY: Elsevier, 1994.

$\mathrm{TiO}_{2}$, modeling, process efficiency.

216. Kawashima, Shinya, and Seigo Nagame. "Inhibition of Streptococcus Mutans Adherence to Pit and Fissure Sealants Containing Powdered Semiconductor TiO.". Shika Igaku 57, no. 2 (1994): $129-40$.

$\mathrm{TiO}_{2}$, disinfection, aqueous phase, application, immobilized $\mathrm{TiO}_{2}$.

217. Kenneke, John F., John L. Ferry, and William H. Glaze. "The Titanium Dioxide-Mediated Photocatalytic Degradation of Chloroalkenes in Water." Photocatalytic Purification and Treatment of Water and Air, eds. David F. Ollis and Hussain Al-Ekabi, 179-9 1, Trace Metals and the Environment, ed. Jerome 0. Nriagu, New York, NY: Elsevier, 1994. $\mathrm{TiO}_{2}$, modeling, aqueous phase, mechanism. 
218. Kerzhentsev, M., C. Guillard, J. M. Herrmann, and P. Pichat. "Titanium Dioxide-Photosensitized Degradation of the Insecticide Tetrachlorvinphos ((z)-2-Chloro-1-(2,4,5Trichlorophenyl)Ethenyl Dimethyl Phosphate)." Photocatalytic Purification and Treatment of Water and Air, eds. David F. Ollis and Hussain Al-Ekabi, 601-, Trace Metals and the Environment, ed. Jerome 0. Nriagu, New York, NY: Elsevier, 1994.

$\mathrm{TiO}_{2}$, aqueous phase, pesticide.

219. Kesselman, Janet M., Amit Kumar, and Nathan S. Lewis. "Fundamental Photoelectrochemistry of Titanium Dioxide and $\mathrm{SrTiO}_{3}$ Applied to Environmental Problems." Photocatalytic Purification and Treatment of Water and Air, eds. David F. Ollis and Hussain Al-Ekabi, 19-37, Trace Metals and the Environment' ed. Jerome 0. Nriagu, New York, NY: Elsevier, 1994. $\mathrm{TiO}_{2}$, other semiconductor, aqueous phase, process efficiency, mechanism.

220. Kesselman, Janet M., Gary A. Shreve, Michael R. Hoffmann, and Nathan S. Lewis. "Flux-Matching Conditions At $\mathrm{TiO}_{2}$ Photoelectrodes: Is Interfacial Electron Transfer to $\mathrm{O}_{2}$ Rate-Limiting in the $\mathrm{TiO}_{2}$-Catalyzed Photochemical Degradation of Organics?" J. Phys. Chem. 98, no. 50 (1994): 13385-95.

$\mathrm{TiO}_{2}$, oxidant, aqueous phase, process efficiency, mechanism, immobilized $\mathrm{TiO}_{2}$.

221. Khan, Shahed U. M., and Bradley D. Craig. "A Model of a Dual Semiconductor Photocatalyst System for the Detoxification of Polluted Water." Proc.-Electrochem. Soc.94-19(Water Purification by Photocatalytic Photoelectrochemical, and Electrochemical Processes) (1994): 352-64. $\mathrm{TiO}_{2}$, other semiconductor, modeling, oxidant, reductant, metallized $\mathrm{TiO}_{2}$, aqueous phase, process efficiency, metal removal.

222. Kidoguchi, Akira, Toshiharu Inaba, and Hidenobu Ito, inventors. "Oxidation of Nitrogen-Containing Compounds.” Japan Mitsui Shipbuilding Eng, assignee. Japan Patent' 06339682 A2. 13 December 1994. CA122:141533.

$\mathrm{TiO}_{2}$, other semiconductor, aqueous phase, application.

223. Kim, Dong Hyun, and Marc A. Anderson. "Performance of Nb-Doped $\mathrm{TiO}_{2}$ Thin Films in Photocatalytic and Photoelectrocatalytic Degradation of Formic Acid." Proc.-Electrochem. Soc. 94-19(Water Purification by Photocatalytic Photoelectrochemical, and Electrochemical Processes) (1994): $342-51$.

$\mathrm{TiO}_{2}$, aqueous phase, modified $\mathrm{TiO}_{2}$, process efficiency.

224. Kitamura, Masaki, inventor. "Manufacture of Photocatalyst." Japan Storage Battery Co Ltd, assignee. Japan Patent, 06246165 A2.6 September 1994. CA122:42586.

$\mathrm{TiO}_{2}$, other semiconductor, gas phase, aqueous phase, modified $\mathrm{TiO}_{2}$, application, immobilized $\mathrm{TiO}_{2}$.

225. Kitamura, Masaki, and Juko Fujita, inventors. "Photocatalyst." Japan Storage Battery Co Ltd, assignee. Japan Patent, 05309267 A2.22 November 1993. CA120(22):281538q.

$\mathrm{TiO}_{2}$, other semiconductor, engineering, modified $\mathrm{TiO}_{2}$, gas phase, aqueous phase, immobilized $\mathrm{TiO}_{2}$.

226. Kitamura, Noboru, Tatsuya Uchida, Hiroyuki Sugimura, and Hiroshi Masuhara. "Microfabrication and Modification for Integrated Chemical Systems." Proc.- Electrochem. Soc. 93-12(Electrochemical Processing of Tailored Materials) (1993): 187-200.

$\mathrm{TiO}_{2}$, metallized $\mathrm{TiO}_{2}$, application, immobilized $\mathrm{TiO}_{2}$. 
227. Kiwi, J. Pulgarin C., P. Peringer, and M. Graetzel. "Beneficial Effects of Heterogeneous Photocatalysis on the Biodegradation of Anthraquinone Sulfonate Observed in Water Treatment." New $J$. Chem. 17, no. 7 (1993): 487-94.

$\mathrm{TiO}_{2}$, aqueous phase, application, solar, biotreatment.

228. Klausner, J. F., A. R. Martin, D. Y. Goswami, and K. S. Schanze. "On the Accurate Determination of Reaction Rate Constants in Batch-Type Solar Photocatalytic Oxidation Facilities." J. Sol. Energy Eng. 116, no. 1 (1994): 19-24.

$\mathrm{TiO}_{2}$, engineering, reactor.

229. Klausner, James F., Andrew R. Martin, and D. Yogi Goswami. "On the Accurate Determination of Reaction Rate Constants in Batch Type Solar Photocatalytic Oxidation Facilities." Solar Engineering 1993, eds. Allan Kirkpatrick and William Worek, 1-8, New York, NY: ASME, 1993. aqueous phase, engineering, solar, $\mathrm{TiO}_{2}$.

230. Ko, Edmond I. "Aerogels As Catalysts and Catalyst Supports." Chemtech April (1993): $31-6$. $\mathrm{TiO}_{2}$, other semiconductor, modified $\mathrm{TiO}_{2}$, immobilized $\mathrm{TiO}_{2}$.

231. Kosaka, Atsushi, Yoshimasa Kodama, and Satoru Kadowaki, inventors. "Manufacture of Regenerable Deodorants." Japan Nippon Soken, and Nippon Denso Co., assignees. Japan Patent, 06170220 A2. 21 June 1994. CA122:137318.

$\mathrm{TiO}_{2}$, other semiconductor, gas phase, aqueous phase, modified $\mathrm{TiO}_{2}$, immobilized $\mathrm{TiO}_{2}$, adsorbent.

232. Koster, T. P. M., J. W. Assink, J. M. Slaager, and C. van der Veen. "Photocatalytic Oxidation of Multi-Component Organochlorine Mixtures in Water." Photocatalytic Purification and Treatment of Water and Air, eds. David F. Ollis and Hussain Al-Ekabi, 613-18, Trace Metals and the Environment' ed. Jerome 0. Nriagu, New York, NY: Elsevier, 1994.

$\mathrm{TiO}_{2}$, aqueous phase, modeling.

233. Koval, Carl A., Foster, Nancy S., Norrof, Karen, Sczechowski, Jeffery G., and Noble, Richard D. "Effects of Controlled Periodic Illumination on the Photoeffrciency of the Photocatalytic Oxidation of Organic Compounds at Titanium Dioxide." Presented at 17th U. S.-Japan Cooperative Seminar on Photoconversion and Photosynthesis Research, La Jolla' CA, 12-14 March 1995, 4 pp.

234. Kuchmy, S. Ya, A. V. Korzhak, S. V. Kulik, A. I. Belous, and A. I. Kryukov. "Photoreductive Dehalogenation of Polychlorobenzenes by Ethanol." Teor. Eksp. Khim. 30, no. 1 (1994): 34-8.

nonaqueous, $\mathrm{TiO}_{2}$, mechanism.

235. Kuhler, Ronald J., Gregory A. Santo, Thomas R. Caudill, Eric A. Betterton, and Robert G. Arnold. "Photoreductive Dehalogenation of Bromoform With Titanium Dioxide-Cobalt Macrocycle Hybrid Catalysts." Environ. Sci. Technol. 27, no. 10 (1993): 2104-11.

$\mathrm{TiO}_{2}$, solar, reductant, aqueous phase, modified $\mathrm{TiO}_{2}$, mechanism.

236. Kuntz, Robert R. "Photocatalytic Reduction of Acetylene by $\left[\mathrm{MoOCl}(\mathrm{Dppe})_{2}\right]^{+} \mathrm{Cl}^{-}$on $\mathrm{TiO}_{2}$." J. Photo\&m. Photobiol., A 84, no. 1 (1994): 75-82.

$\mathrm{TiO}_{2}$, aqueous phase, modified $\mathrm{TiO}_{2}$. 
237. Kuznetsova, N. A., and 0. L. Kaliya. "Photoinduced Oxidation of Rhodamine 6Zh in Titanium Dioxide Aqueous Suspension." Zh. Fit Khim. 67, no. 7 (1993): 1492-5.

$\mathrm{TiO}_{2}$, aqueous phase, dye.

238. Lai, Cuiwei, and Thomas E. Mallouk. "A New Approach to the Photochemical Trifluoromethylation of Aromatic Compounds." J.Chem. Soc., Chem. Commun., no. 17 (1993): 1359-61. Benzene and Its Derivatives, $\mathrm{TiO}_{2}$, nonaqueous, application.

239. Larson, Sheldon A., and John L. Falconer. "Characterization of $\mathrm{TiO}_{2}$ Photocatalysts Used in Trichloroethene Oxidation.” Appl. Catal., B 4, no. 4 (1994): 325-42.

$\mathrm{TiO}_{2}$, gas phase, aqueous phase, mechanism, catalyst reactivation, catalyst characterization.

240. Larson, Sheldon A., and John L. Falconer. "Characterization of Titanium Dioxide Used in Liquid-Phase and Gas-Phase Photooxidation of Trichloroethylene." Photocatalytic Purification and Treatment of Water and Air, eds. David F. Ollis and Hussain Al-Ekabi, 473-9, Trace Metals and the Environment' ed. Jerome 0. Nriagu, New York, NY: Elsevier, 1994.

$\mathrm{TiO}_{2}$, gas phase, aqueous phase, process efficiency, mechanism, adsorption, catalyst reactivation.

241. Lawless, Darren F. "Photophysical Studies on Ultra-Small Semiconductor Particles: CdS Quantum Dots, Doped and Undoped $\mathrm{TiO}_{2}$, and Silver Halides." Ph.D. diss., Corcordia Univ., Can., 1993. $\mathrm{TiO}_{2}$, other semiconductor, modified $\mathrm{TiO}_{2}$, mechanism.

242. Lee, W., Y. R. Do, K. Dwight, and A. Wold. "Enhancement of Photocatalytic Activity of Titanium(N) Oxide With Molybdenum(VI) Oxide.” Mater. Res. Bull. 28, no. 11 (1993): 1127-34. $\mathrm{TiO}_{2}$, aqueous phase, modified $\mathrm{TiO}_{2}$.

243. Lee, W., H. S. Shen, K. Dwight' and A. Wold. "Effect of Silver on the Photocatalytic Activity of Titania." J. Solid State Chem. 106, no. 2 (1993): 288-94.

$\mathrm{TiO}_{2}$, metallized $\mathrm{TiO}_{2}$, aqueous phase, modified $\mathrm{TiO}_{2}$, metal removal.

244. Lee, Wan In. "Study of the Photocatalytic Property of Titanium (IV) Oxide (Gold, Ultrasonic Nebulization, Flame Hydrolysis)." Ph.D. diss., Brown Univ., Providence, RI, USA, 1993. $\mathrm{TiO}_{2}$, metallized $\mathrm{TiO}_{2}$, modified $\mathrm{TiO}_{2}$, catalyst characterization.

245. Lepore, Giuseppe P., Cooper H. Langford, Jana Vichova, and Antonin Jr. Vlcek. "Photochemistry and Picosecond Absorption Spectra of Aqueous Suspensions of a Polycrystalline Titanium Dioxide Optically Transparent in the Visible Spectrum." J. Photochem. Photobiol., A 75, no. 1 (1993): 67-75.

$\mathrm{TiO}_{2}$, aqueous phase, modified $\mathrm{TiO}_{2}$, process efficiency, mechanism.

246. Lepore, Giuseppe P., Bhuvan C. Pant' and Cooper H. Langford. 'Limiting Quantum Yield Measurements for the Disappearance of 1-Propanol and Propanal: an Oxidative Reaction Study Employing a TiO 2 -Based Photoreactor." Can. J. Chem. 71, no. 12 (1993): 2051-g. $\mathrm{TiO}_{2}$, reactor, aqueous phase, modified $\mathrm{TiO}_{2}$, process efficiency, mechanism, immobilized $\mathrm{TiO}_{2}$, adsorption. 
247. Lepore, Giuseppe P., Antonin Jr. Vlcek, and Cooper H. Langford. "The Photocatalytic Oxidation of Propanol by Titanium Dioxide." Photocatalytic Purification and Treatment of Water and Air, eds. David F. Ollis and Hussain Al-Ekabi, 95-109, Trace Metals and the Environment, ed. Jerome 0. Nriagu, New York, NY: Elsevier, 1994. $\mathrm{TiO}_{2}$, aqueous phase, modified $\mathrm{TiO}_{2}$, process efficiency, mechanism, adsorption.

248. Li, Tian, and Xushi Yan. "Photocatalytic Oxidation for Drinking Water Purification." Water Treat. 9, no. 2 (1994): 119-26.

$\mathrm{TiO}_{2}$, aqueous phase, application.

249. Li, Tian, and Xushi Yan. "Study on Removing Organochlorine Compounds in Water by Photocatalytic Oxidation Method." Shanghai Huanjing Kexue 11, no. 12 (1992): 1 1-14,7.

$\mathrm{TiO}_{2}$, aqueous phase, application, catalyst reactivation.

250. Li, Tian, Xushi Yan, and Weiyi Xu. "Photocatalytic Oxidation of Humic Acid in Aqueous Solution." Shuichuli Jishu 20, no. 1 (1994): 51-5. $\mathrm{TiO}_{2}$, aqueous phase, application.

251. Lichtin, Norman N., and M. Avudaithai. "Application of Photocatalytic Oxidative Degradation to Industrial Waste Streams."Photochem. Photoelectrochem. Convers. Storage Sol. Energy, Proc. Znt. Conf., 9th, ed. Zhao Wu Tian, 97-112, Beijing, Peop. Rep. China: Int. Acad. Publ., 1993. $\mathrm{TiO}_{2}$, oxidant, aqueous phase, application, process efficiency, cost.

252. Lichtin, Norman N., Musuthami Avudaithai, and Elliot Berman. "Relative Reactivity of Aqueous and Vaporized VOCs to Photocatalytic Oxidative Mineralization Over $\mathrm{TiO}_{2}$." Proc. Electrochem. Soc.94-19(Water Purification by Photocatalytic, Photoelectrochemical, and Electrochemical Processes) (1994): 320- 1.

$\mathrm{TiO}_{2}$, reactor, gas phase, aqueous phase, mechanism, modeling, adsorption.

253. Lichtin, Norman N., Muthusami Avudaithai, Elliot Berman, and Junchang Dong. "Photocatalytic Oxidative Degradation of Vapors of Some Organic Compounds Over $\mathrm{TiO}_{2}$." Res. Chem. Intermed. 20, no. 7 (1994): 755-8 1.

$\mathrm{TiO}_{2}$, gas phase, modified $\mathrm{TiO}_{2}$, process efficiency.

254. Lin, Lufei, and Robert R. Kuntz. "Competative Photocatalytic Reduction of $\mathrm{H}+$ and $\mathrm{C} 2 \mathrm{H} 2$ by Mo2S4(S2C2H4)2(2-) on Colloidal TiO 2." J. Photochem. Photobiol. A: Chem. 66 (1992): 245-5 1. $\mathrm{TiO}_{2}$, reductant, aqueous phase, modified $\mathrm{TiO}_{2}$, process efficiency.

255. Lin, Wen Yuan, Chang Wei, and Krishnan Rajeshwar. "Photocatalytic Reduction and Immobilization of Hexavalent Chromium At Titanium Dioxide in Aqueous Basic Media." J. Electrochem. Soc. 140, no. 9 (1993): 2477-82.

$\mathrm{TiO}_{2}$, oxidant, aqueous phase, application, metal removal.

256. Liidner, Martin, Detlef W. Bahnemann, Bemd Hirthe, and Wolf-D. Griebler. "Solar Water Detoxification: Novel $\mathrm{TiO}_{2}$ Powders As Highly Active Photocatalysts. " Solar Engineering 1995, eds. William B. Stine, Tadayoshi Tanaka' and David E. Claridge, 399-408, New York, NY: ASME, 1995. $\mathrm{TiO}_{2}$, modeling, aqueous phase, modified $\mathrm{TiO}_{2}$, process efficiency, mechanism, catalyst reactivation, anion inhibition. 
257. Linkous, Clovis A., Nazim Z. Muradov, and Ali T-Raissi. "A Perspective on Photo- and Electrochemical Detoxification of Aqueous Nitrate Esters." Proc. - Electrochem. Soc. 94-19(Water purification by Photocatalytic Photoelectrochemical, and Electrochemical Processes) (1994): 309-19. $\mathrm{TiO}_{2}$, aqueous phase, application, process efficiency, immobilized $\mathrm{TiO}_{2}$.

258. Lmsebigler, Amy L., Guangquan Lu, and John T. Yates Jr. "Photocatalysis on $\mathrm{TiO}_{2}$ Surfaces: Principles, Mechanisms, and Selected Results." Chem. Rev. 95, no. 3 (1995): 735-58.

$\mathrm{TiO}_{2}$, gas phase, aqueous phase, modified $\mathrm{TiO}_{2}$, process efficiency, mechanism, immobilized $\mathrm{TiO}_{2}$, metal removal, catalyst characterization.

259. Litter, Marta I., and Jose A. Navio. "Comparison of the Photocatalytic Efficiency of $\mathrm{TiO}_{2}$, Iron Oxides and Mixed Ti(IV)-Fe(III) Oxides: Photodegradation of Oligocarboxylic Acids." J. Photochem. Photobiol., A 84, no. 2 (1994): 183-93.

$\mathrm{TiO}_{2}$, other semiconductor, modified $\mathrm{TiO}_{2}$, aqueous phase.

260. Liu, Xinsheng, Kai Kong Iu, and J. Kerry Thomas. "Preparation, Characterization and Photoreactivity of Titanium(IV) Oxide Encapsulated in Zeolites." J. Chem. Soc., Faraday Trans. 89, no. 11 (1993): 1861-5. $\mathrm{TiO}_{2}$, aqueous phase, modified $\mathrm{TiO}_{2}$, immobilized $\mathrm{TiO}_{2}$, adsorbent.

261. Lodha, Anita' Jatinder Kaur, Pinki B. Punjabi, and Suresh C. Ameta. "Photocatalytic Reaction of Ethambutol on Zinc Oxide Powder." Rev. Roum. Chim. 39, no. 5 (1994): 583-6. other semiconductor, oxidant' aqueous phase, mechanism.

262. Love, Jonathan G., A. James McQuillan, and David A. Buckingham. "In-Situ IR Reflection Absorption Spectroscopy (IRRAS) During W-Irradiation of Ferrocyanide Adsorbed on $\mathrm{TiO}_{2} . "$ Bull. Electrochem. 9, no. 5-7 (1993): 277-9.

$\mathrm{TiO}_{2}$, aqueous phase, modified $\mathrm{TiO}_{2}$, mechanism, immobilized $\mathrm{TiO}_{2}$, metal removal.

263. Lu, G., A. Linsebigler, and J. T.Yates Jr. "Photooxidation of $\mathrm{CH}_{3} \mathrm{Cl}$ on $\mathrm{TiO}_{2}$ (110): A Mechanism Not Involving $\mathrm{H}_{2}$ O." J. Phys. Chem.99, no. 19 (1995): 7626-3 1.

$\mathrm{TiO}_{2}$, gas phase, mechanism, adsorption, catalyst characterization.

264. Lu, Guangquan, Amy Lmsebigler, and John T. Yates Jr. "The Photochemical Identification of Two Chemisorption States for Molecular Oxygen on $\mathrm{TiO}_{2}(110)$." J. Chem. Phys. 102, no. 7 (1995): 3005-8.

$\mathrm{TiO}_{2}$, gas phase, mechanism, immobilized $\mathrm{TiO}_{2}$, adsorption.

265. Lu, Ming-Chun, Gwo-Dong Roam, Jong-Nan Chen, and C. P. Huang. "Factors Affecting the Photocatalytic Degradation of Dichlorvos Over Titanium Dioxide Supported on Glass." J. Photochem. Photobiol., A 76, no. 1-2 (1993): 103-10.

Agrochemical, $\mathrm{TiO}_{2}$, pesticide, aqueous phase, application.

266. Lyons, Carol, Craig S. Turchi, and David Gratson. Solving Widespread Low-Concentration VOC Air Pollution Problems: Gas-Phase Photocatalytic Oxidation Answers the Needs of Many Small Businesses, NREL,TP-473-7569. National Renewable Energy Laboratory, Golden, CO, 1995. DE95004059.

$\mathrm{TiO}_{2}$, gas phase, application, cost. 
267. Maeda, Yukitoshi, Susumu Kato, and Atsushi Matsushita' inventors. "Pretreatment With Photocatalyst for Biological Treating Wastewaters.” Japan Maeda Kosen Kk, assignee. Japan Patent, 06182363 A2.5 July 1994. CA121:237788.

$\mathrm{TiO}_{2}$, engineering, reactor, solar, oxidant' aqueous phase, modified $\mathrm{TiO}_{2}$, application, immobilized $\mathrm{TiO}_{2}$, biotreatment.

268. Maeda, Yukitoshi, Susumu Kato, and Atsushi Matsushita' inventors. "Treatment of Wastewater by Biological Treatment Using Photocatalysts for Pretreatment.” Japan Maeda Kosen Kk, assignee. Japan Patent, 07060269 A2.7 March 1995. CA122:273233.

$\mathrm{TiO}_{2}$, other semiconductor, engineering, reactor, aqueous phase, application, immobilized $\mathrm{TiO}_{2}$, biotreatment.

269. Magrini, K. A., R. M. Goggin, A. S. Watt' A. M. Taylor, and A. L. Baker. "Improving Catalyst Performance for Solar-Based Photocatalytic Oxidation of Organics." Solar Engineering 1994, eds. David E. Klett, Roy E. Hogan, and Tadayoshi Tanaka' 163-9, 1994.

$\mathrm{TiO}_{2}$, metallized $\mathrm{TiO}_{2}$, aqueous phase, modified $\mathrm{TiO}_{2}$.

270. Magrini, Kimberly A., A. Watt' and B. Rinehart. "Photocatalyst Evaluation for Solar Based Aqueous Organic Oxidation.” Solar Engineering 1995, eds. William B. Stine, Tadayoshi Tanaka, and David E. Claridge, 415-20, New York, NY: ASME, 1995.

$\mathrm{TiO}_{2}$, metallized $\mathrm{TiO}_{2}$, aqueous phase, modified $\mathrm{TiO}_{2}$, ferrioxalate.

271. Maillard-Dupuy, Catherine, Chantal Guillard, Henri Courbon, and Pierre Pichat. "Kinetics and Products of the $\mathrm{TiO}_{2}$ Photocatalytic Degradation of Pyridine in Water." Environ. Sci. Technol.28, no. 12 (1994): 2176-83.

$\mathrm{TiO}_{2}$, pesticide, aqueous phase, application, mechanism.

272. Maillard-Dupuy, Catherine, Chantal Guillard, and Pierre Pichat. "The Degradation of Nitrobenzene in Water by Photocatalysis Over $\mathrm{TiO}_{2}$ : Kinetics and Products; Simultaneous Elimination of Benzamide or Phenol or Pb2+ Cations." New J. Chem. 18, no. 8-9 (1994): 941-8.

$\mathrm{TiO}_{2}$, oxidant, aqueous phase, process efficiency, metal removal, adsorption.

273. Malinka, E. A., and G. L. Kamolov. "Photocatalytic Hydrogen Evolution From Aqueous Pt/TiO 2 Suspension in the Presence of Oxalic Acid." React. Kinet. Catal.Lett. 52, no. 1 (1994): 13-8. $\mathrm{TiO}_{2}$, reductant, metallized $\mathrm{TiO}_{2}$, aqueous phase, mechanism.

274. Mansour, M., and E. A. Feicht. "Transformation of Chemical Contaminants by Biotic and Abiotic Processes in Water and Soil." Chemosphere 28, no. 2 (1994): 323-32.

$\mathrm{TiO}_{2}$, solar, pesticide, aqueous phase.

275. Mao, Y., C. Schoeneich, and K. D. Asmus. "Radical Mediated Degradation Mechanisms of Halogenated Organic Compounds As Studied by Photocatalysis At Titanium Dioxide and by Radiation Chemistry." Photocatalytic Purification and Treatment of Water and Air, eds. David F. Ollis and Hussain Al-Ekabi, 49-66, Trace Metals and the Environment' ed. Jerome 0. Nriagu, New York, NY: Elsevier, 1994. $\mathrm{TiO}_{2}$, modeling, oxidant, aqueous phase, process efficiency, mechanism.

276. Martin, Carlos A., Miguel A. Baltanas, and Alberto E. Cassano. "Photocatalytic Reactors. I. Optical Behavior of Titanium Oxide Particulate Suspensions." J. Photochem. Photobiol., A 76, no. 3 (1993): 199-208.

$\mathrm{TiO}_{2}$, reactor, modeling. 
277. Martin, Scot T., H. Herrmann, W. Choi, and Michael R. Hoffmann. "Photochemical Destruction of Chemical Contaminants on Quantum-Sized Semiconductor Particles. " Solar Engineering 1995, eds. William B. Stine, Tadayoshi Tanaka, and David E. Claridge, 409-13, New York, NY: ASME, 1995.

$\mathrm{TiO}_{2}$, other semiconductor, solar, oxidant, reductant, aqueous phase, modified $\mathrm{TiO}_{2}$, process efficiency, mechanism, immobilized $\mathrm{TiO}_{2}$, metal removal.

278. Martin, Scot T., Hartmut Herrmann, Wonyong Choi, and Michael R. Hoffmann. "Time-Resolved Microwave Conductivity. Part 1. $\mathrm{TiO}_{2}$ Photoreactivity and Size Quantization." J. Chem. Soc., Faraday Trans. 90, no. 21 (1994): 3315-22.

$\mathrm{TiO}_{2}$, other semiconductor, aqueous phase, modified $\mathrm{TiO}_{2}$, process efficiency, mechanism.

279. Martin, Scot T., Colin L. Morrison, and Michael R. Hoffmann. "Photochemical Mechanism of Size-Quantized Vanadium-Doped TiO 2 Particles." J. Phys. Chem. 98, no. 51 (1994): 13695-704.

$\mathrm{TiO}_{2}$, aqueous phase, modified $\mathrm{TiO}_{2}$, mechanism, process efficiency.

280. Masuda, Ryuji, Koichi Kawashima, Wataru Takahashi, Masayuki Murabayashi, and Kiminori Ito, inventors. "Photocatalysts for Treatment of Harmful Substances and Its Apparatus." Japan Nippon Muki Kk, assignee. Japan Patent, 06320010 A2.22 November 1994. CA122:118763. $\mathrm{TiO}_{2}$, immobilized $\mathrm{TiO}_{2}$, modified $\mathrm{TiO}_{2}$.

281. Masuda, Ryuji, Koichi Kawashima, Wataru Takahashi, Masayuki Murabayashi, and Kiminori Ito, inventors. "Photocatalysts and Apparatus for Heavy Metal Ion Removal From Solutions." Japan Nippon Muki Kk, assignee. Japan Patent, 06320011 A2.22 November 1994. CA122:114046. $\mathrm{TiO}_{2}$, immobilized $\mathrm{TiO}_{2}$, metal removal, aqueous phase

282. Matsumoto, Yasumichi, Michio Obata, and Jukishi Hombo. "Photocatalytic Reduction of Carbon Dioxide on P-Type $\mathrm{CaFe}_{2} \mathrm{O}_{4}$ Powder." J. Phys. Chem. 98, no. 11(1994): 2950-51.

other semiconductor, reductant, aqueous phase, mechanism.

283. Matsunaga, Tadashi, inventor. "Process for Killing Cells." Tokyo Japan Kabashiki Kalsya Advanced Kalhatsu Kenkyujo, assignee. United States Patent, 4708038.29 November 1988. disinfection, immobilized $\mathrm{TiO}_{2}$, other semiconductor, aqueous phase, gas phase.

284. Matsunaga, Tadashi, Ryuzo Tomoda, Toshiaki Nakjima, Noriyuki Nakumura, and Tamotsu Komine. "Continuous-Sterilization System That Uses Photo-Semiconductor Powders." Appl. Environ. Microbiol. 54, no. 6 (1988): 1330-3.

$\mathrm{TiO}_{2}$, disinfection, aqueous phase, process efficiency, immobilized $\mathrm{TiO}_{2}$.

285. Matsushita, Atsushi, inventor. "Manufacture of Titanium Oxide Plate Material for Photocatalyst.” Japan Maeda Kosen Kk, assignee. Japan Patent, 06210170 A2.2 August 1994. CA121:241566.

$\mathrm{TiO}_{2}$, aqueous phase, immobilized $\mathrm{TiO}_{2}$. 
286. Matthews, R. W. "Environment: Photochemical and Photocatalytic Processes. Degradation of Organic Compounds." Photochemical Conversion and Storage of Solar Energy, Proceedings of the 8th International Conference on Photochemical Conversion and Storage of Solar Energy, eds. E. Pelizzetti, and M. Schiavello, 427-3, Dordrecht: Kluwer Academic Publishers, 1991. $\mathrm{TiO}_{2}$, other semiconductor, solar, oxidant, aqueous phase, modified $\mathrm{TiO}_{2}$, application, mechanism, process efficiency, immobilized $\mathrm{TiO}_{2}$, photochemistry.

287. Matthews, Ralph, inventor. "Apparatus and Method for Determining Concentration of Organic Carbon in High-Purity Water." Australia Commonwealth Scientific and Industrial Research Organization, assignee. Australia Patent' 657477 B2. 16 March 1995. CA122:322120.

$\mathrm{TiO}_{2}$, other semiconductor, reactor, aqueous phase, application, immobilized $\mathrm{TiO}_{2}$.

288. Mehos, Mark, Craig S. Turchi, Jim Pacheco, A. J. Boegel, Tim Merrill, and Tim Stanley. Pilot-Scale Study of the Solar Detoxification of VOC-Contaminated Groundwater, NREL,TP-432-498 1. NREL, Golden, CO, 1992. DE92016405.

$\mathrm{TiO}_{2}$, engineering, reactor, solar, aqueous phase, application, process efficiency.

289. Mehos, Mark, Tom Williams, and Craig S. Turchi. Overview of Solar Detoxification Activities in the United States, NREL,TP-471-7262. NREL, Golden, CO, 1994. DE95000264. $\mathrm{TiO}_{2}$, solar, gas phase, aqueous phase, application, SI Program.

290. Memming, Ruediger. "Photoinduced Charge Transfer Processes At Semiconductor Electrodes and Particles." Topics in Current Chemistry 169 (1994): 105-81. $\mathrm{TiO}_{2}$, other semiconductor, solar, oxidant' reductant, metallized $\mathrm{TiO}_{2}$, aqueous phase, modified $\mathrm{TiO}_{2}$, application, process efficiency, mechanism, immobilized $\mathrm{TiO}_{2}$, metal removal.

291. Mezza, Michel, and Brigitte Berthomieu, inventors. "Photodegradable Propylene and Ethylene Polymer Compositions."France Patent, 2692583 Al. 24 December 1993. CA121:302239. nonaqueous, $\mathrm{TiO}_{2}$, solar, application, immobilized $\mathrm{TiO}_{2}$.

292. Micic, Olga I., Yuenian Zhang, Keith R. Cromack, Alexander D. Trifunac, and Marion C. Thumauer. "Trapped Holes on $\mathrm{TiO}_{2}$ Colloids Studied by Electron Paramagnetic Resonance." J. Phys. Chem. 97 (1993): 7277-83.

$\mathrm{TiO}_{2}$, other semiconductor, reductant, aqueous phase, mechanism.

293. Micic, Olga I., Yuenian Zhang, Keith R. Cromack, Alexander D. Trifunac, and Marion Thumauer. "Photoinduced Hole Transfer From $\mathrm{TiO}_{2}$ to Methanol Molecules in Aqueous Solution Studied by Electron Paramagnetic Resonance." J. Phys. Chem.97, no. 50 (1993): 13284-8.

$\mathrm{TiO}_{2}$, oxidant, reductant, aqueous phase, mechanism.

294. Milis, A., J. Peral, and X. Domenech. "Kinetics of Photocatalytic Oxidation of Sulfite-Nitrite Mixtures in Aqueous $\mathrm{TiO}_{2}$ Suspensions." Oxid. Commun. 17, no. 3-4 (1994): 163-9. $\mathrm{TiO}_{2}$, aqueous phase, mechanism, metallized $\mathrm{TiO}_{2}$, oxidant.

295. Milis, Ali, Jose Peral, Xavier Domenech, and J. A. Navio. "Heterogeneous Photocatalytic Oxidation of Nitrite Over Iron-Doped $\mathrm{TiO}_{2}$ Samples." J. Mol. Catal. 87, no. 1 (1994): 67-74.

$\mathrm{TiO}_{2}$, reductant, aqueous phase, modified $\mathrm{TiO}_{2}$, adsorption. 
296. Militemo, Simonetta, Luigi Campanella, and Giancarlo Crescentini. "Photocatalytic Degradation of Selected Chlorophenols and Pesticides by Immobilized Polyaniline and $\mathrm{TiO}_{2}$."Mobility Degrad. Xenobiot., Proc. - Simp. Pestic. Chem.Attilio A. M. Del Re, 431-O. Lucca, Italy: G. Biagini, 1993. $\mathrm{TiO}_{2}$, pesticide, aqueous phase, modified $\mathrm{TiO}_{2}$, immobilized $\mathrm{TiO}_{2}$.

297. Mills, Andrew, Ahmed Belghazi, Richard H. Davies, David Worsley, and Sian Morris. "A Kinetic Study of the Bleaching of Rhodamine 6G Photosensitized by Titanium Dioxide." J. Photochem.

Photobiol., A 79, no. 1-2 (1994): 131-9.

$\mathrm{TiO}_{2}$, aqueous phase.

298. Mills, Andrew, and Richard Davies. "Activation Energies in Semiconductor Photocatalysis for Water Purification: the 4-Chlorophenol- $\mathrm{TiO}_{2}-\mathrm{O}_{2}$ Photosystem." J. Photochem.

Photobiol., A 85, no. 1-2 (1995): 173-8.

299. Mills, Andrew, and Richard Davies. "The Photomineralization of Reactive Black 5 Sensitized by Titanium Dioxide: a Study of the Initial Kinetics of Dye Photobleaching." Photocatalytic Purification and Treatment of Water and Air, eds. David F. Ollis and Hussain Al-Ekabi, 595-600, Trace Metals and the Environment' ed. Jerome 0. Nriagu, New York, NY: Elsevier, 1994. $\mathrm{TiO}_{2}$, aqueous phase, process efficiency.

300. Mills, Andrew, Richard H. Davies, and David Worsley. "Water Purification by Semiconductor Photocatalysis." Chem.Soc. Rev. 22, no. 6 (1993): 417-25. $\mathrm{TiO}_{2}$, aqueous phase, application.

301. Mills, Andrew, Peter Douglas, Alison Green, and Geraint Williams. "Diffusion- and Activation-Controlled Photoredox Reactions Sensitized by Colloidal Semiconductors."Electrochem. Colloids Dispersions, [Symp. Electrochem. Microheterog. Fluids] eds. Raymond A. Texter and John Mackay, 385-97, New York, N.Y: VCH, 1992.

$\mathrm{TiO}_{2}$, other semiconductor, aqueous phase, process efficiency, mechanism.

302. Mills, Andrew, Carolyn E. Holland, Richard H. Davies, and David Worsley. "Photomineralization of Salicylic Acid: a Kinetic Study." J. Photochem. Photobiol., A 83, no. 3 (1994): 257-63.

$\mathrm{TiO}_{2}$, modeling, aqueous phase, adsorption.

303. Mills, Andrew, and Sian Morris. "Photomineralization of 4-Chlorophenol Sensitized by Titanium Dioxide: a Study of the Effect of Annealing the Photocatalyst At Different Temperatures." $J$. Photochem. Photobiol., A 71, no. 3 (1993): 285-9.

$\mathrm{TiO}_{2}$, aqueous phase, modified $\mathrm{TiO}_{2}$, process efficiency.

304. Mills, Andrew, David Worsley, and Richard H. Davies. "Effect of ph on the Stability of $\mathrm{TiO}_{2}$ Coatings on Glass Photocatalysis Reactors for Water Purification." J. Chem. Soc., Chem. Commun., no. 23 (1994): 2677-8.

$\mathrm{TiO}_{2}$, aqueous phase, immobilized $\mathrm{TiO}_{2}$.

305. Mills, German, and Michael R. Hoffmann. "Photocatalytic Degradation of Pentachlorophenol on Titanium Dioxide Particles: Identification of Intermediates and Mechanism of Reaction.” Environ. Sci. Technol. 27, no. 8 (1993): 1681-9. $\mathrm{TiO}_{2}$, oxidant, aqueous phase, process efficiency, mechanism. 
306. Minero, C., E. Pelizzetti, S. Malato, and J. Blanco. "Large Solar Plant Photocatalytic Water Decontamination: Degradation of Pentachlorophenol." Chemosphere 26, no. 12 (1993): 2103-19. $\mathrm{TiO}_{2}$, solar, engineering, reactor, oxidant, aqueous phase.

307. Minero, C., E. Pelizzetti, P. Piccinini, and M. Vincenti. "Photocatalyzed Transformation of Nitrobenzene on $\mathrm{TiO}_{2}$ and $\mathbf{Z n O}$." Chemosphere 28, no. 6 (1994): 122944.

$\mathrm{TiO}_{2}$, other semiconductor, aqueous phase, mechanism.

308. Minero, C., E. Pelizzetti, R. Terzian, and N. Serpone. "Reactions of Hexafluorobenzene and Pentafluorophenol Catalyzed by Irradiated $\mathrm{TiO}_{2}$ in Aqueous Suspensions." Langmuir 10, no. 3 (1994): 692-8.

$\mathrm{TiO}_{2}$, aqueous phase, mechanism.

309. Minoura, Hideki. "Photocatalytic Reactions on Semiconductor Films." Kikan Kagaku Sosetsu 23 (1994): 79-85. nonaqueous, $\mathrm{TiO}_{2}$, application, immobilized $\mathrm{TiO}_{2}$.

310. Minsker, L., C. Pulgarin, P. Peringer, and J. Kiwi. "Integrated Approach Useful in the Mineralization of Nonbiodegradable, Toxic P-Nitrotoluenesulfonic Acid Vi Photocatalytic-Biological Process." New J. Chem. 18, no. 7 (1994): 793-800.

$\mathrm{TiO}_{2}$, oxidant' aqueous phase, biotreatment.

311. Mitani, Michiharu, Takuya Kiriyama, and Tomoaki Kuratate. "Addition Reaction of Polychloro Compounds to Carbon-Carbon Multiple Bonds Catalyzed by Semiconductor Particles Under Photoirradiation." J. Org. Chem. 59, no. 6 (1994): 1279-82.

nonaqueous, $\mathrm{TiO}_{2}$, mechanism, reductant.

312. Miyoshi, Noriomi, Kazuteru Shinkai, Toshiharu Sasamoto, and Hitomi Kawakami, inventors. "Method and Apparatus for Removing Harmful Gas From Exhaust Gas." Ltd. Japan Fuji Electric Co., assignee. Europe Patent' 591920 A2. 13 April 1994. CA120:306375.

$\mathrm{TiO}_{2}$, other semiconductor, gas phase, modified $\mathrm{TiO}_{2}$, application, adsorbent, immobilized $\mathrm{TiO}_{2}$.

313. Monaci, Anna' Aldo La Ginestra, and Pasquale Patrono. "Zirconium Phosphates As Photocatalysts." J. Photochem. Photobiol. A: Chem. 83 (1994): 63-7.

nonaqueous, $\mathrm{TiO}_{2}$, other semiconductor, mechanism.

314. Murabayashi, Masalyuki, Kiminori Itoh, Seiji Suzuki, Koichi Kawashima, and Ryuji Masuda. "Photocatalytic Degradation of Chloroform by Using Titanium Dioxide Thin Films Coated on Solid Substrate." Proc.-Electrochem. Soc. 93-18, Proceedings of the Symposium on Environmental Aspects of Electrochemistry and Photoelectrochemistry (1993): 13 1-6.

$\mathrm{TiO}_{2}$, aqueous phase, immobilized $\mathrm{TiO}_{2}$.

315. Murabayashi, Masayuki, Kiminori Itoh, Koichi Kawashima, Ryuji Masuda, and Seiichi Suzuki. "Photocatalytic Degradation of Chloroform With Titanium Dioxide Coated Glass Fiber Cloth." Photocatalytic Purification and Treatment of Water and Air, eds. David F. Ollis and Hussain Al-Ekabi, 783-8, Trace Metals and the Environment' ed. Jerome 0. Nriagu, New York, NY: Elsevier, 1994. $\mathrm{TiO}_{2}$, aqueous phase, immobilized $\mathrm{TiO}_{2}$. 
316. Murabayashi, Masayuki, and Kazuo Okamura "Degradation of Chlorinated Organic Compounds Using Fixed Photocatalyst." Yosui to Haisui 36, no. 10 (1994): 877-2.

$\mathrm{TiO}_{2}$, gas phase, immobilized $\mathrm{TiO}_{2}$, modified $\mathrm{TiO}_{2}$.

317. Muradov, Nazim Z. "Solar Detoxification of Nitroglycerin-Contaminated Water Using Immobilized Titania." Sol. Energy 52, no. 3 (1994): 283-8.

$\mathrm{TiO}_{2}$, solar, metallized $\mathrm{TiO}_{2}$, aqueous phase, immobilized $\mathrm{TiO}_{2}$.

318. Muradov, Nazim Z., and Ali T-Raissi. "Titania-Catalyzed, Photooxidative Detoxification of Nitroglycerin-Contaminated Airborne VOCs." Chem. Oxid. Volume Date 1993, no. 3 (1994): 172-82. $\mathrm{TiO}_{2}$, gas phase, immobilized $\mathrm{TiO}_{2}$.

319. Murasawa, Sadao. "Recent Applications of Photocatalysis." Denki Kagaku Oyobi Kogyo Butsuri Kagaku 63, no. 1 (1995): 9-13.

$\mathrm{TiO}_{2}$, disinfection, gas phase, aqueous phase, application.

320. Nair, Maya' Zhenghao Luo, and Adam Heller. "Rates of Photocatalytic Oxidation of Crude Oil on Salt Water on Buoyant, Cenosphere-Attached Titanium Dioxide." Ind. Eng. Chem. Res. 32, no. 10 (1993): 23 18-23.

$\mathrm{TiO}_{2}$, engineering, aqueous phase, modified $\mathrm{TiO}_{2}$, immobilized $\mathrm{TiO}_{2}$.

321. Nargiello, Maria, and Ted Herz. "Physical-Chemical Characteristics of P-25 Making It Extremely Suited As the Catalyst in Photodegradation of Organic Compounds." Photocatalytic

Purification and Treatment of Water and Air, eds. David F. Ollis and Hussain Al-Ekabi, 801-7, Trace Metals and the Environment' ed. Jerome 0. Nriagu, New York, NY: Elsevier, 1994.

$\mathrm{TiO}_{2}$, gas phase, aqueous phase, modified $\mathrm{TiO}_{2}$, cost.

322. Nishida, K., and S. Ohgaki. "Photolysis of Aromatic Chemical Compounds in Aqueous $\mathrm{TiO}_{2}$ Suspensions." Water Sci Technol. 30, no. 9 (1994): 39-46.

$\mathrm{TiO}_{2}$, pesticide.

323. Nishiguchi, H., and M. Anpo. "Photochemical Properties of Benzophenone Adsorbed on Ti-Al and Ti-Si Binary Oxides."Chem. Funct. Dyes, Proc. Znt. Symp., 2nd, eds. Z. Yoshida, and Y. Shirota, 153-8, Tokyo, Japan: Mita Press, 1993.

$\mathrm{TiO}_{2}$, other semiconductor.

324. Nogueira, Raquel F. P., and Wilson F. Jardim. "Photodegradation of Methylene Blue: Using Solar Light and Semiconductor (Titanium Dioxide)." J. Chem. Educ. 70, no. 10 (1993): 861-2. Education, $\mathrm{TiO}_{2}$, reactor, solar, aqueous phase.

325. Nosaka, Yoshio. "Mechanism of Photocatalytic Reactions on Semiconductor Particles." Presented at 17th U.S.-Japan Cooperative Seminar on Photoconversion and Photosynthesis Research, La Jolla, CA, 12-14 March 1995, 3 pp.

326. Nosaka, Yoshio. "Photocatalytic Aspects of Ultrasmall Semiconductor Particles." Kikan Kagaku Sosetsu 23 (1994): 62-8.

$\mathrm{TiO}_{2}$, other semiconductor, aqueous phase, modified $\mathrm{TiO}_{2}$, process efficiency, mechanism. 
327. Notthakun, Sawang, John C. Crittenden, David W. Hand, David L. Perram, and Michael E. Mullins. "Regeneration of Adsorbents Using Heterogeneous Advanced Oxidation." J. Environ. Eng. 1 19, no. 4 (1993): 695-714.

$\mathrm{TiO}_{2}$, solar, metallized $\mathrm{TiO}_{2}$, aqueous phase, immobilized $\mathrm{TiO}_{2}$, adsorbent.

328. O'Shea' Kevin E., and Claudia Cardona "Hammett Study on the $\mathrm{TiO}_{2}$-Catalyzed Photooxidation of Para-Substituted Phenols. A Kinetic and Mechanistic Analysis." J. Org. Chem. 59, no. 17 (1994): 5005-9.

$\mathrm{TiO}_{2}$, aqueous phase, mechanism.

329. Obee, Timothy N., and Robert T. Brown. "TiO 2 Photocatalysis for Indoor Air Applications: Effects of Humidity and Trace Contaminant Levels on the Oxidation Rates of Formaldehyde, Toluene, and 1,3-Butadiene." Environ. Sci. Technol. 29, no. 5 (1995): 1223-3 1. $\mathrm{TiO}_{2}$, gas phase, application, immobilized $\mathrm{TiO}_{2}$.

330. Oberg, V., D. Y. Goswami, and G. Svedberg. "On Photocatalytic Detoxification of Water Containing Volatile Organic Compounds. " Solar Engineering 1994, eds. David E. Klett, Roy E. Hogan, and Tadayoshi Tanaka' 147-53, 1994.

$\mathrm{TiO}_{2}$, engineering, reactor, solar, oxidant, aqueous phase, application, adsorption.

331. Oeste, Franz Dietrich, inventor. "Photocatalytic Purification of Waste Gases." Germany PCP Photocatalytic Purification GmbH, assignee. Germany Patent' 4320218 Al. 21 July 1994.

CA121:186142.

$\mathrm{TiO}_{2}$, other semiconductor, engineering, reactor, gas phase, modified $\mathrm{TiO}_{2}$, aqueous phase, adsorption.

332. Ogawa, Shunjiro, Mamiko Tanigawa, Michiko Fujioka, and Yukiko Hanasaki.

"Photocatalyzed Destruction of Humic Acid in Aqueous Semiconductor Suspension and Diminution of Its Trihalomethane Production Potential." Jpn. J. Toxicol. Environ. Health 41, no. 1 (1995): 7.

$\mathrm{TiO}_{2}$, aqueous phase, application.

333. Ogawa, Takatoshi, Toshio Saito, Kenichi Unno, Kan Hasegawa, Yasuhiko Yoshioka, Noburo Tsubochi, Seiichi Hosoiri, Tooru Katayama, Akira Fujishima, and Kazuhito Hashimoto, inventors. "Materials for Construction of Hospitals for Preventing Infections." Japan Takenaka Komuten Co, assignee. Japan Patent, 07000462 A2.6 January 1995. CA122:170302.

$\mathrm{TiO}_{2}$, other semiconductor, metallized $\mathrm{TiO}_{2}$, disinfection, gas phase, application, IAQ.

334. Ogawa, Talcatoshi, Kenichi Unno, Hidehiko Nakasaki, Takeshi Hiromatsu, Norio Igawa, Shigetaka Magara, Toshio Saito, Akira Fujishima, and Kazuhito Hashimoto, inventors. "Apparatus for Photochemical Synthesis of Methanol." Japan Takenaka Komuten Co, assignee. Japan Patent, 07033697 A2.3 February 1995. CA122:239181.

$\mathrm{TiO}_{2}$, other semiconductor, reactor, engineering, solar, reductant, aqueous phase, modified $\mathrm{TiO}_{2}$, immobilized $\mathrm{TiO}_{2}$.

335. Ogawa, Takatoshi, Yasuhiko Yoshioka, Nobuo Tsubouchi, Toshio Saito, Tamotsu Hasegawa, Akira Fujishima, and Kazuhito Hashimoto, inventors. "Architectural Material Using Metal Oxide Exhibiting Photocatalytic Activity." Japan Takenaka Corp., assignee. Canada Patent, 2106510 AA. 23 March 1994. CA121:140271.

$\mathrm{TiO}_{2}$, other semiconductor, solar, metallized $\mathrm{TiO}_{2}$, disinfection, gas phase, aqueous phase, modified $\mathrm{TiO}_{2}$, application, IAQ, immobilized $\mathrm{TiO}_{2}$. 
336. Ohgaki, Shinichiro, and Kei Nishida. "Photocatalytic Degradation of Aromatic Compounds Using Titanium Dioxide." Yosui to Haisui 36, no. 10 (1994): 858-63.

$\mathrm{TiO}_{2}$, pesticide, aqueous phase.

337. Ohnishi, Hideyuki, Michio Matsumura, Hiroshi Tsubomura, and Makoto Iwasaki. "Bleaching of Lignin Solution by a Photocatalyzed Reaction on Semiconductor Photocatalysts." I\&EC Research 28, no. 6 (1989): 719-24.

$\mathrm{TiO}_{2}$, other semiconductor, metallized $\mathrm{TiO}_{2}$, aqueous phase, modified $\mathrm{TiO}_{2}$, application, mechanism.

338. Ohtani, Bunsho. "Recent Progress in Research of Semiconductor Photocatalysis. Materials, Reactions, and Mechanism." Shokubai 36, no. 7 (1994): 515-23.

$\mathrm{TiO}_{2}$, other semiconductor, aqueous phase, mechanism.

339. Ohtani, Fumiaki. "Photodegradation of Polyolefms by Titanium Oxide Fine Particles." Kobunshi Kako 42, no. 5 (1993): 226-31.

Synthetic High Polymers, $\mathrm{TiO}_{2}$, oxidant, modified $\mathrm{TiO}_{2}$, application, mechanism, immobilized $\mathrm{TiO}_{2}$.

340. Ollis, David F. "Comparative Aspects of Advanced Oxidation Processes." Presented at ACS Symposium - Emerging Technologies for Hazardous Waste Destruction, Atlanta' GA, October 1991, $21 \mathrm{pp}$.

341. Ollis, David F. "Solar-Assisted Photocatalysis for Water Purification: Issues, Data, Questions." Photochemical Conversion and Storage of Solar Energy, Proceedings of the 8th International Conference on Photochemical Conversion and Storage of Solar Energy, eds. E. Pelizzetti and M. Schiavello, 593-622, Dordrecht: Kluwer Academic Publishers, 1991.

$\mathrm{TiO}_{2}$, engineering, reactor, solar, oxidant, aqueous phase, application, process efficiency, mechanism, immobilized $\mathrm{TiO}_{2}$, cost.

342. Ollis, David F., Ezio Pelizzetti, and Nick Serpone. "Heterogeneous Photocatalysis in the Environment: Application to Water Purification." Photocatalysis Fundamentals and Applications, eds. Nick Serpone and Ezio Pelizzetti, 603-37. New York: John Wiley \& Sons, 1989.

$\mathrm{TiO}_{2}$, other semiconductor, engineering, reactor, modeling, aqueous phase, mechanism, metal removal.

343. Ollis, David F., Ezio Pelizzetti, and Nick Serpone. "Photocatalyzed Destruction of Water Contaminants." Environ. Sci. Technol. 25, no. 9 (1991): 1522-9.

$\mathrm{TiO}_{2}$, other semiconductor, engineering, solar, modeling, aqueous phase, application, process efficiency, mechanism, metal removal.

344. Onoda, Kinji, Yasukiyo Ogose, and Shoichiro Izumi, inventors. "A Redox Method and Membrane-Separated Photoelectrochemical Cell.” Ltd. Shiken, assignee. Japan Patent' 6328895.6 February 1988. CA109:63262b.

$\mathrm{TiO}_{2}$, other semiconductor, reactor, disinfection, aqueous phase, application, immobilized $\mathrm{TiO}_{2}$, metal removal.

345. Oozora, Hiroyuki, inventor. "Photochemical Conversion of Carbon Dioxide." Mitsubishi Heavy Ind Ltd, assignee. Japan Patent, 05146671 A2.15 June 1993. CA119(20):213896j.

$\mathrm{TiO}_{2}$, reductant, aqueous phase, modified $\mathrm{TiO}_{2}$, immobilized $\mathrm{TiO}_{2}$. 
346. Pacheco, J. E., M. R. Prairie, and L. Yellowhorse. "Photocatalytic Destruction of Chlorinated Solvents in Water With Solar Energy." J. Sol. Energy Eng. 115, no. 3 (1993): 123-9. $\mathrm{TiO}_{2}$, reactor, solar, aqueous phase, engineering.

347. Pacheco, Kelli, Andrew S. Watt' and Craig S. Turchi. "Solar Detoxification of Water: Outdoor Testing of Prototype Photoreactors." ASME,ASES Joint Solar Energy Conference, eds. Allan Kirkpatrick and William Worek, 43-49, New York, NY: ASME, 1993.

$\mathrm{TiO}_{2}$, engineering, reactor, solar, aqueous phase, immobilized $\mathrm{TiO}_{2}$.

348. Palmisano, L., M. Schiavello, A. Sclafani, C. Martin, I. Martin, and V. Rives. "Surface Properties of Iron-Titania Photocatalysts Employed for 4-Nitrophenol Photodegradation in Aqueous $\mathrm{TiO}_{2}$ Dispersion." Catal. Lett. 24, no. 3-4 (1994): 303-15.

$\mathrm{TiO}_{2}$, aqueous phase, modified $\mathrm{TiO}_{2}$, mechanism, adsorption.

349. Palmisano, L., M. Schiavello, A. Sclafani, G. Martra, E. Borello, and S. Coluccia. "Photocatalytic Oxidation of Phenol on $\mathrm{TiO}_{2}$ Powders. A Fourier Transform Infrared Study." Appl.Catal., $B$ 3, no. 2-3 (1994): 117-32.

$\mathrm{TiO}_{2}$, gas phase, mechanism, adsorption.

350. Palmisano, Leonardo, Vincenzo Augugliaro, Renzo Campostrini, and Mario Schiavello. "A Proposal for the Quantitative Assessment of Heterogeneous Photocatalytic Processes." J. Catal. 143, no. 1 (1993): 149-54.

$\mathrm{TiO}_{2}$, other semiconductor, modeling, aqueous phase, process efficiency, modified $\mathrm{TiO}_{2}$.

351. Pandit, Goutam K., Srikumar Pal, and Asit K. Das. "Photocatalytic Degradation of Pendimethalin in the Presence of Titanium Dioxide." J. Agric. Food Chem. 43, no. 1 (1995): 171-4. nonaqueous, application, pesticide.

352. Papp, J., S. Soled, K. Dwight' and A. Wold. "Surface Acidity and Photocatalytic Activity of $\mathrm{TiO}_{2}, \mathrm{WO}_{3} / \mathrm{TiO}_{2}$, and $\mathrm{MoO}_{3} / \mathrm{TiO}_{2}$ Photocatalysts." Chem. Mater. 6, no. 4 (1994): 496-500.

$\mathrm{TiO}_{2}$, aqueous phase, modified $\mathrm{TiO}_{2}$, process efficiency.

353. Papp, Jennifer Ellen. "Titanium(IV) Oxide Photocatalysts With Palladium and Surface Acidity and Photocatalytic Activity of Titanium(IV) Oxide, Tungsten(VI) Oxide,Titanium(IV) Oxide, and Molybdenum(VI) Oxide,Titanium(IV) Oxide Photocatalysts." Ph.D. diss., Brown Univ., USA, 1994. $\mathrm{TiO}_{2}$, modified $\mathrm{TiO}_{2}$, catalyst characterization.

354. Pehkonen, Simo O., Ron Siefert, Yigal Erel, Sam Webb, and Michael R. Hoffmann. "Photoreduction of Iron Oxyhydroxides in the Presence of Important Atmospheric Organic Compounds." Environ. Sci. Technol. 27 (1993): 2056-62.

other semiconductor, solar, reductant, atmosphere.

355. Pelizzetti, E., C. Minero, E. Borgarello, L. Tinucci, and N. Serpone. "Photocatalytic Activity and Selectivity of Titania Colloids and Particles Prepared by the Sol-Gel Technique:

Photooxidation of Phenol and Atrazine." Langmuir 9, no. 11(1993): 2995-3001.

$\mathrm{TiO}_{2}$, pesticide, modified $\mathrm{TiO}_{2}$, aqueous phase, immobilized $\mathrm{TiO}_{2}$.

356. Pelizzetti, E., C. Minero, and V. Carlin. "Photoinduced Degradation of Atrazine Over Different Metal Oxides." New J. Chem. 17, no. 4 (1993): 315-19.

$\mathrm{TiO}_{2}$, other semiconductor, aqueous phase, modified $\mathrm{TiO}_{2}$, pesticide. 
357. Pelizzetti, E., C. Minero, P. Piccinini, and M. Vincenti. "Phototransformations of Nitrogen Containing Organic Compounds Over Irradiated Semiconductor Metal Oxides. Nitrobenzene and Atrazine Over Titania and Zinc Oxide." Coord. Chem. Rev. 125, no. 1-2 (1993): 183-93.

$\mathrm{TiO}_{2}$, other semiconductor, pesticide, aqueous phase.

358. Pelizzetti, E., C. Minero, E. Pramauro, and M. Vincenti. "Recent Issues on Environmental Detoxification by Solar Photocatalysis." Photochem. Photoelectrochem. Convers. Storage Sol. Energy,

Proc. Int.Conf., 9th, ed. Zhao Wu Tian, 2 17-33, Beijing, Peop. Rep. China: Int. Acad. Publ., 1993.

$\mathrm{TiO}_{2}$, reactor, solar, aqueous phase, application.

359. Pelizzetti, E., C. Minero, M. Sega, and M. Vincenti. "Formation and Disappearance of Biphenyl Derivatives in the Photocatalytic Transformation of 1,2,4-Trichlorobenzene on Titanium Dioxide." Photocatalytic Purification and Treatment of Water and Air, eds. David F. Ollis and Hussain Al-Ekabi, 291-300, Trace Metals and the Environment, ed. Jerome 0. Nriagu, New York, NY: Elsevier, 1994.

$\mathrm{TiO}_{2}$, aqueous phase, mechanism.

360. Pelizzetti, Ezio, Claudio Minero, Hisao Hidaka, and Nick Serpone. "Photocatalytic Processes for Surfactant Degradation." Photocatalytic Purification and Treatment of Water and Air, eds. David F. Ollis and Hussain Al-Ekabi, 261-73, Trace Metals and the Environment, ed. Jerome 0. Nriagu, New York, NY: Elsevier, 1994.

$\mathrm{TiO}_{2}$, solar, aqueous phase.

361. Peral, Jose, and David F. Ollis. "Photocatalyst Deactivation: Oxidation of Decamethyl-Tetrasiloxane, Pyrrole, Indole and Dimethyl Sulfide. " Photocatalytic Purification and Treatment of Water and Air, eds. David F. Ollis and Hussain Al-Ekabi, 741-5, Trace Metals and the Environment, ed. Jerome 0. Nriagu, New York, NY: Elsevier, 1994.

$\mathrm{TiO}_{2}$, gas phase, application.

362. Peris-Cardells, E., J. Terol, A. R. Mauri, M. de la Guardia, and E. Pramauro. "Continuous Flow Photocatalytic Degradation of Carbaryl in Aqueous Media." J. Environ. Sci. Health, Part B B28, no. 4 (1993): 431-5.

Agrochemical, $\mathrm{TiO}_{2}$, reactor, pesticide, aqueous phase.

363. Perraud, R., M. Papazian, L. Khidour, B. Pouyet, and J. P. Percherancier. "Suitable Process for Degradation of Malodorous Molecules." Recents Prog. Genie Procedes 7, no. 30 (1993): 3 89-94.

$\mathrm{TiO}_{2}$, engineering, gas phase, immobilized $\mathrm{TiO}_{2}, \mathrm{IAQ}$.

364. Peyton, Gary R., and David W. DeBerry. Feasibility of Photocatalytic Oxidation for Wastewater Clean-Up and Reuse, OWRT,RU-81,1.SumX, Corp., Austin, TX, 1981.

$\mathrm{TiO}_{2}$, other semiconductor, solar, aqueous phase, application, process efficiency.

365. Pham, Hien N., Thomas McDowell, and Ebtisam Wilkins. "Photocatalytically-Mediated Disinfection of Water Using $\mathrm{TiO}_{2}$ As a Catalyst and Spore-Forming Bacillus Pumilus As a Model." J. Environ. Sci. Health, Part A: Environ. Sci. Eng. Toxic Hazard. Subst. Control A30, no. 3 (1995): 627-36.

$\mathrm{TiO}_{2}$, disinfection, aqueous phase, application. 
366. Pichat, P. "Photocatalysis: Heterogeneous Regime. Catalysts, Adsorption and New Techniques." Photochemical Conversion and Storage of Solar Energy, Proceedings of the 8th International Conference on Photochemical Storage Of Solar Energy, eds. E. Pelizzetti and M. Schiavello, 277-93, Dordrecht: Kluwer Academic Publishers, 1991.

$\mathrm{TiO}_{2}$, other semiconductor, gas phase, aqueous phase, modified $\mathrm{TiO}_{2}$, mechanism, immobilized $\mathrm{TiO}_{2}$, adsorption, isotope exchange.

367. Pichat, Pierre, Jean-Marie Herrmann, Jean Disdier, and Marie-Noelle Mozzanega. "Photocatalytic Oxidation of Propene Over Various Oxides At 320 K. Selectivity." J. Phys. Chem. 83, no. 24 (1979): 3122-6.

$\mathrm{TiO}_{2}$, other semiconductor, gas phase, process efficiency, mechanism.

368. Polo, E., R. Amadelli, V. Carassiti, and A. Maldotti. "Photocatalytic Oxygenation of Hydrocarbons on Titania,Iron-Porphyrin Hybrid Catalysts.” Stud. Surf. Sci. Catal. 78, Heterogeneous Catalysis and Fine Chemicals III (1993): 409-16.

$\mathrm{TiO}_{2}$, nonaqueous, modified $\mathrm{TiO}_{2}$.

369. Porter, J. F., and P. L. Yue. "Simple Supported Photocatalysts." Photocatalytic Purification and Treatment of Water and Air, eds. David F. Ollis and Hussain Al-Ekabi, 759-64, Trace Metals and the Environment' ed. Jerome 0. Nriagu, New York, NY: Elsevier, 1994.

$\mathrm{TiO}_{2}$, engineering, reactor, modeling, solar, aqueous phase, modified $\mathrm{TiO}_{2}$, immobilized $\mathrm{TiO}_{2}$.

370. Poulios, I., and A. Avranas. "Heterogeneous Photocatalytic Degradation of the Cationic Surfactants Cetyldimethylbenzylammonium Chloride and Cetylpyridinium Chloride." NATO ASI Ser., Ser. E 225, Chemical Reactor Technology for Environmentally Safe Reactors and Products (1992): 609- 15. $\mathrm{TiO}_{2}$, reactor, metallized $\mathrm{TiO}_{2}$, aqueous phase.

371. Prairie, M. R., B. M. Stange, and L. R Evans. "Titanium Dioxide Photocatalysis for the Destruction of Organics and the Reduction of Heavy Metals." Photocatalytic Purification and Treatment of Water and Air, eds. David F. Ollis and Hussain Al-Ekabi, 353-63, Trace Metals and the Environment' ed. Jerome 0. Nriagu, New York, NY: Elsevier, 1994.

$\mathrm{TiO}_{2}$, oxidant, reductant, aqueous phase, metal removal.

372. Prairie, Michael R., Lindsey R. Evans, and Sheryl L. Martinez. "Destruction of Organics and Removal of Heavy Metals in Water Via $\mathrm{TiO}_{2}$ Photocatalysis." Chem. Oxid. Volume Date 1992, no. 2 (1994): 428-1.

$\mathrm{TiO}_{2}$, oxidant, reductant, aqueous phase, metal removal.

373. Prairie, Michael R., Lindsey R. Evans, Bertha M. Stange, and Sheryl L. Martinez. "An Investigation of Titanium Dioxide Photocatalysis for the Treatment of Water Contaminated With Metals and Organic Chemicals.” Environ. Sci. Technol. 27, no. 9 (1993): 1776-82.

$\mathrm{TiO}_{2}$, oxidant, reductant, aqueous phase, metal removal.

374. Pramauro, Edmondo, Alessandra Bianco Prevot, Vincenzo Augugliaro, and Leonardo Palmisano. "Photocatalytic Treatment of Laboratory Wastes Containing Aromatic Amines." Analyst 120, no. 2 (Cambridge, U. K.) (1995): 237-42.

$\mathrm{TiO}_{2}$, aqueous phase. 
375. Pramauro, Edmondo, Marco Vincenti, Vincenzo Augugliaro, and Leonardo Palmisano. "Photocatalytic Degradation of Monuron in Aqueous Titanium Dioxide Dispersions." Environ. Sci. Technol. 27, no. 9 (1993): 1790-5.

$\mathrm{TiO}_{2}$, pesticide, aqueous phase, mechanism.

376. Presley, R. W. "Characterization of Titanium Dioxide: Factors Affecting Photocatalytic Performance."Sci. Technol. Alliance, Mater. Conf. '93, ed. Jagannathan Sankar, 211-16. Lancaster, PA: Technomic, 1993.

$\mathrm{TiO}_{2}$, aqueous phase, modified $\mathrm{TiO}_{2}$.

377. Rader, W. Scott' Ljiljana Solujic, E. B. Milosavljevic, J. L. Hendrix, and J. H. Nelson. "Photochemistry of Aqueous Solutions of Dicyanomercury(II) and Potassium Tetracyanomercurate(II)." J. Sol. Energy Eng. 116, no. 3 (1994): 125-9.

$\mathrm{TiO}_{2}$, solar, oxidant' aqueous phase, application, metal removal.

378. Rader, W. Scott, Ljiljana Solujic, Emil B. Milosavljevic, James L. Hendrix, and John H. Nelson. "Sunlight-Induced Photochemistry of Aqueous Solutions of Hexacyanoferrate(II) and -(III) Ions." Environ. Sci. Technol. 27, no. 9 (1993): 1875-9.

$\mathrm{TiO}_{2}$, solar, oxidant, reductant, aqueous phase, metal removal.

379. Rader, W. Scott, Ljiljana Solujic, Emil B. Milosavljevic, James L. Hendrix, and John H. Nelson. "Photochemistry of Aqueous Solutions of Dicyanomercury(II) and Potassium Tetracyanomercurate(II). " Solar Engineering 1994, eds. David E. Klett, Roy E. Hogan, and Tadayoshi Tanaka' 111-6, 1994.

$\mathrm{TiO}_{2}$, solar, aqueous phase, application, metal removal.

380. Rao, N. N., Sangeeta Dube, and P. Natarajan. "Photocatalytic Degradation of Some Chlorohydrocarbons in Aqueous Suspensions of $\mathrm{M0}_{3}, \mathrm{TiO}_{2}(\mathrm{M}=\mathrm{Mo}$ or W)." Photocatalytic Purification and Treatment of Water and Air, eds. David F. Ollis and Hussain Al-Ekabi, 695-700, Trace Metals and the Environment' ed. Jerome 0. Nriagu, New York, NY: Elsevier, 1994.

$\mathrm{TiO}_{2}$, modified $\mathrm{TiO}_{2}$, pesticide.

381. Rao, N. Nageswara, and P. Natarajan. "Particulate Models in Heterogeneous Photocatalysis." Current Science 66, no. 10 (1994): 742-52.

$\mathrm{TiO}_{2}$, other semiconductor, solar, aqueous phase, application, mechanism, metal removal.

382. Raupp, G. B., and J. A. Dumesic. "Adsorption of CO, C02, H2, and $\mathbf{H} 20$ on Titania Surfaces With Different Oxidation States.” J. Phys. Chem. 89 (1985): 5240-6.

$\mathrm{TiO}_{2}$, gas phase, mechanism, immobilized $\mathrm{TiO}_{2}$, adsorption.

383. Raupp, Gregory B., and Craig T. Junio. "Photocatalytic Oxidation of Oxygenated Air Toxics." Appl. Surf. Sci. 72, no. 4 (1993): 321-7.

$\mathrm{TiO}_{2}$, gas phase, process efficiency.

384. Raupp. Gregory B., Craig T. Junio, R. K. Mallela, and L. A. Phillips. "Destruction of Organics in Gaseous Streams Over W-Excited Titania." Proc., Annu. Meet. - Air Waste Manage. Assoc., Paper No. 92-26.10, 1992.

gas phase, $\mathrm{TiO}_{2}$, reactor. 
385. Richard, C. "Photocatalytic Reduction of Benzoquinone in Aqueous $\mathbf{Z n O}$ or $\mathrm{TiO}_{2}$ Suspensions." New J. Chem. 18, no. 4 (1994): 443-5.

$\mathrm{TiO}_{2}$, other semiconductor, oxidant, reductant, aqueous phase.

386. Richard, C. "Regioselectivity of Oxidation by Positive Holes (h+) in Photocatalytic Aqueous Transformations." J. Photochem. Photobiol., A 72, no. 2 (1993): 179-82.

$\mathrm{TiO}_{2}$, other semiconductor, oxidant, reductant, aqueous phase, mechanism.

387. Richard, C., and P. Boule. "Photocatalytic Oxidation of Phenolic Derivatives: Influence of $\mathrm{OH}$ and $\mathrm{H}+$ on the Distribution of Products." New J.Chem. 18, no. 4 (1994): 547-2.

$\mathrm{TiO}_{2}$, aqueous phase, mechanism.

388. Richard, C., A. M. Matre, and P. Boule. "Photocatalytic Transformation of 2,5-Furandimethanol in Aqueous $\mathrm{ZnO}$ Suspensions." J. Photochem. Photobiol. A: Chem. 66 (1992): 225-34. other semiconductor.

389. Riegel, Georg, and James R. Bolton. "Photocatalytic Efficiency Variability in $\mathrm{TiO}_{2}$ Particles." J. Phys. Chem. 99, no. 12 (1995): 4215-24.

$\mathrm{TiO}_{2}$, modeling, aqueous phase, modified $\mathrm{TiO}_{2}$, process efficiency, mechanism.

390. Rizzuti, L., and A. Brucato. "Recent Developments in Heterogeneous Photoreactor Modelling." Photochemical Conversion and Storage of Solar Energy, Proceedings of the 8th International Conference on Photochemical Conversion and Storage of Solar Energy, eds. E. Pelizzetti and M. Schiavello, 561-74, Dordrecht: Kluwer Academic Publishers, 1991.

$\mathrm{TiO}_{2}$, other semiconductor, engineering, reactor, modeling, solar, application.

391. Robertson, Michael K., and Robert B. Henderson, inventors. "Fluid Purification." London Ontario Canada Nutech Energy Systems Inc., assignee. United States Patent, 5032241.16 July 1991. $\mathrm{TiO}_{2}$, other semiconductor, reactor, disinfection, application, immobilized $\mathrm{TiO}_{2}$.

392. Rose, T. L., J. Ibechem, and B. Aurian-Blajeni. "Photocatalytic Destruction of Dichloroacetic Acid in Aqueous Solutions At Temperatures Above 100 C." Proc.-Electrochem. Soc. 94-19(Water Purification by Photocatalytic Photoelectrochemical, and Electrochemical Processes) (1994): 298-308.

$\mathrm{TiO}_{2}$, aqueous phase, reductant.

393. Ross, Hardow, Juergen Bendig, and Stefan Hecht. "Sensitized Photocatalytical Oxidation of Terbutylazine." Sol. Energy Mater. Sol. Cells 33, no. 4 (1994): 475-8 1.

$\mathrm{TiO}_{2}$, solar, aqueous phase, modified $\mathrm{TiO}_{2}$, mechanism, reductant.

394. Ruppert, G., K. Hofstadler, R. Bauer, and G. Heisler. "Heterogeneous and Homogeneous Photoassisted Wastewater Treatment." Proc.-Indian Acad. Sci., Chem. Sci. 105, no. 6 (1993): 393-7. $\mathrm{TiO}_{2}$, oxidant, aqueous phase, mechanism.

395. Saiga, Tetsuyuki, inventor. "Method for Preparation of 1,1 '-Dialkyl-4,4'-Dipyridinium Salts." Japan Shingijutsu Kaihatsu Jigyodan, and Saiga Tetsuyuki, assignees. Japan Patent, 06263734 A2. 20 September 1994. CA122:187400.

$\mathrm{TiO}_{2}$, other semiconductor, solar, nonaqueous, application. 
396. Saiga, Tetsuyuki, inventor. "Semiconductor Modified by Insoluble Polyviologen Derivatives." Japan Shingijutsu Kaihatsu Jigyodan, and Saiga Tetsuyuki, assignees. Japan Patent, 06268199 A2.22 September 1994. CA122:304360.

nonaqueous, $\mathrm{TiO}_{2}$, other semiconductor, modified $\mathrm{TiO}_{2}$.

397. Saika, Tetsuyuki, Tomokazu Iyoda, and Takeo Shimidzu. "Preparation of PolyviologenModified $\mathrm{TiO}_{2}$ by Photocatalytic Polymerization of Bis(4-Cyano-1 -Pyridinio)-p-Xylene Dibromide."

Chem. Lett., no. 12 (1993): 2025-8.

$\mathrm{TiO}_{2}$, nonaqueous, modified $\mathrm{TiO}_{2}$.

398. Sakai, Hideki, Ryo Baba, Kazuhito Hashimoto, and Akira Fujishima. "Separate

Monitoring of Reaction Products Formed At Oxidation and Reduction Sites of $\mathrm{TiO}_{2}$ Photocatalysts Using a Microelectrode.” J. Electroanal. Chem. 379, no. 1-2 (1994): 199-205.

$\mathrm{TiO}_{2}$, oxidant, reductant, aqueous phase, mechanism.

399. Sakai, Hideki, Ru Xiong Cai, Ryo Baba, Kazuhito Hashimoto, Yoshinobu Kubota, and Akira Fujishima "Detection of Intermediates Formed At the Surface of Photoexcited Titanium Dioxide Film Electrode Using Microelectrode." Photocatalytic Purification and Treatment of Water and Air, eds. David F. Ollis and Hussain Al-Ekabi, 651-7, Trace Metals and the Environment' ed. Jerome 0. Nriagu, New York, NY: Elsevier, 1994.

$\mathrm{TiO}_{2}$, aqueous phase, mechanism, immobilized $\mathrm{TiO}_{2}$.

400. Sakura, Makoto, inventor. "Moisture-Resistant Catalysts Decomposing Ethylene Formed During Storage of Vegetables and Fruits.” Japan Nitsuki Yunibaasaru Kk, assignee. Japan Patent, 07016473 A2. 20 January 1995. CA122:289437.

$\mathrm{TiO}_{2}$, gas phase, modified $\mathrm{TiO}_{2}$, application, IAQ, adsorbent, immobilized $\mathrm{TiO}_{2}$.

401. Sakurada, Tsukasa. "Sterilization of Microorganisms by Photo-Semiconductor Cloth. On Some Applications of Function Material." Hyomen Gijutsu 41, no. 10 (1990): 1008-1.

$\mathrm{TiO}_{2}$, metallized $\mathrm{TiO}_{2}$, disinfection, aqueous phase, modified $\mathrm{TiO}_{2}$, process efficiency, immobilized $\mathrm{TiO}_{2}$.

402. Sampath, Srinivasan, Hiroyuki Uchida, and Hiroshi Yoneyama "Photocatalytic

Degradation of Gaseous Pyridine Over Zeolite-Supported Titanium Dioxide." J. Catal. 149, no. 1 (1994): 189-94.

$\mathrm{TiO}_{2}$, gas phase, modified $\mathrm{TiO}_{2}$, immobilized $\mathrm{TiO}_{2}$, adsorbent.

403. Sanchez, Beningo, Manuel Romero, Alfonso Vidal, Begona Fabrellas, Jesus Blanco, and Pedro Avila "Destruction of Toluene and Xylene Using Concentrated Solar Photons." Solar Engineering 1994, eds. David E. Klett, Roy E. Hogan, and Tadayoshi Tanaka' 123-9, 1994.

$\mathrm{TiO}_{2}$, solar, gas phase, immobilized $\mathrm{TiO}_{2}$.

404. Sato, Shigeyuki, and Kenichiro Suzuki, inventors. "Metal Oxide-Based Photocatalysts and the Preparation.” Japan Toyoda Chuo Kenkyusho Kk, assignee. Japan Patent, 06182205 A2.5 July 1994.

CA121:239300.

$\mathrm{TiO}_{2}$, metallized $\mathrm{TiO}_{2}$, gas phase, modified $\mathrm{TiO}_{2}$, application, immobilized $\mathrm{TiO}_{2}$.

405. Sato, Shinri, and Toru Kadowaki. "Photocatalytic Activities of Metal Oxide

Semiconductors for Oxygen Isotope Exchange and Oxidation Reactions." J. Catal. 106 (1987): 295-300. $\mathrm{TiO}_{2}$, gas phase, modified $\mathrm{TiO}_{2}$, mechanism. 
406. Sato, Shinri, A. Sobczynski, J. M. White, A. J. Bard, A. Campion, M. A. Fox, T. E. Mallouk, and S. E. Webber. "Photochemical Properties of Ultrathin $\mathrm{TiO}_{2}$ Films Prepared by Chemical Vapor Deposition.” J. Photochem. Photobiol. A: Chem. 50 (1989): 283-90.

$\mathrm{TiO}_{2}$, metallized $\mathrm{TiO}_{2}$, aqueous phase, modified $\mathrm{TiO}_{2}$, immobilized $\mathrm{TiO}_{2}$.

407. Sauer, Michael L., and David F. Ollis. "Acetone Oxidation in a Photocatalytic Monolith Reactor." J. Catal. 149, no. 1 (1994): 81-91.

$\mathrm{TiO}_{2}$, reactor, gas phase, application, IAQ, immobilized $\mathrm{TiO}_{2}$, adsorbent, adsorption.

408. Sauve, Genevieve, Prashant V. Kamat, and Rodney S. Ruoff. "Excited Triplet and Reduced Forms of $\mathrm{C}_{84} . "$ J. Phys. Chem. 99, no. 7 (1995): 2162-5.

$\mathrm{TiO}_{2}$, aqueous phase, modified $\mathrm{TiO}_{2}$.

409. Schaper, K., and D. Hesse. "Photocatalytic Flue Gas Cleaning."IChemE Res. Event, Two-Day Symp., 380-2, Rugby, UK: Inst. Chem. Eng., 1994.

$\mathrm{TiO}_{2}$, reductant, gas phase, application, immobilized $\mathrm{TiO}_{2}$, catalyst reactivation.

410. Schaper, Klaus, and Diethard Hesse. "Problem of Catalyst Deactivation During Photocatalytic Flue Gas Treatment." Chem.-Ing.-Tech. 66, no. 1 (1994): 86-9.

$\mathrm{TiO}_{2}$, reductant, gas phase, catalyst reactivation.

411. Schertz, Paul, Dee Kelly, and Luke Lammert. Analysis of Cost of Generating or Capturing Ultraviolet Light for Photocatalytic Water Detoxification Systems. Final Report for NREL Subcontract No. AF-2-11252-1, NREL Subcontract No. AF-2-11252-1 Final Report.

cost, engineering, lamps.

412. Schmelling, D. C., and K. A Gray. "Feasibility of Photocatalytic Degradation of TNT As a Single or Integrated Treatment Process." Photocatalytic Purification and Treatment of Water and Air, eds. David F. Ollis and Hussain Al-Ekabi, 625-3 1, Trace Metals and the Environment' ed. Jerome 0. Nriagu, New York, NY: Elsevier, 1994.

$\mathrm{TiO}_{2}$, aqueous phase, biotreatment.

413. Schneider, M., E. D. Duff, T. Mallat, M. Wildberger, and A. Baiker. "High Surface Area Platinum-Titania Aerogels: Preparation, Structural Properties, and Hydrogenation Activity." J. Catal. 147 (1994): 500-14.

$\mathrm{TiO}_{2}$, metallized $\mathrm{TiO}_{2}$, modified $\mathrm{TiO}_{2}$, catalyst characterization.

414. Schwitzgebel, Jorg, Ekerdt, J. G., Gerischer, H., and Heller, Adam. "Role of the Oxygen Molecule and of the Photogenerated Electron in $\mathrm{TiO}_{2}$ Photocatalyzed Air Oxidation Reactions." Presented at 17th U. S.-Japan Cooperative Seminar on Photoconversion and Photosynthesis Research, La Jolla' CA, 12-14 March 1995, 4 pp.

aqueous phase, application, immobilized $\mathrm{TiO}_{2}$, mechanism, oxident

415. Sclafani, A., A. Brucato, and L. Rizzuti. "Mass Transfer Limitations in a Packed Bed Photoreactor Used for Phenol Removal." Photocatalytic Purification and Treatment of Water and Air, eds. David F. Ollis and Hussain Al-Ekabi, 533-45, Trace Metals and the Environment' ed. Jerome 0. Nriagu, New York, NY: Elsevier, 1994.

$\mathrm{TiO}_{2}$, reactor, aqueous phase, mechanism, immobilized $\mathrm{TiO}_{2}$. 
416. Sclafani, A., L. Palmisano, M. Schiavello, V. Augugliaro, S. Coluccia, and L. Marchese. "The Photodecomposition of Ethanoic Acid Adsorbed by Semiconductor and Insulator Oxides. Part 1. Pure Oxides." New J.Chem. 12 (1988): 129-35.

$\mathrm{TiO}_{2}$, aqueous phase other semiconductors.

417. Sclafani, A., L. Palmisano, M. Schiavello, V. Augugliaro, S. Coluccia, and L. Marchese. "The Photodecomposition of Ethanoic Acid Adsorbed by Semiconductor and Insulator Oxides. Part 2. Mixed Insulator Oxides." New J.Chem. 12 (1988): 137-41.

$\mathrm{TiO}_{2}$, aqueous phase, modified $\mathrm{TiO}_{2}$.

418. Sczechowski, J. G., C. A. Koval, and R. D. Noble. "Improved Photoefficiencies for Titanium Dioxide Photocatalytic Reactors Through the Use of Controlled Periodic Illumination. " Photocatalytic Purification and Treatment of Water and Air, eds. David F. Ollis and Hussain Al-Ekabi, 645-50, Trace Metals and the Environment, ed. Jerome 0. Nriagu, New York, NY: Elsevier, 1994. $\mathrm{TiO}_{2}$, reactor, engineering, aqueous phase, process efficiency.

419. Sczechowski, Jeffrey G. "Applying Controlled Periodic Illumination in a Taylor Vortex Reactor to Increase the Photoeffrciency in Heterogeneous Photocatalysis." Solar Engineering 1995, eds. William B. Stine, Tadayoshi Tanaka' and David E. Claridge, 459-66, New York, NY: ASME, 1995. $\mathrm{TiO}_{2}$, engineering, reactor, modeling, aqueous phase, process efficiency, mechanism.

420. Sczechowski, Jeffrey G., Carl A. Koval, and Richard D. Noble. "Evidence of Critical Illumination and Dark Recovery Times for Increasing the Photoefficiency of Aqueous Heterogeneous Photocatalysis." J. Photochem. Photobiol., A 74, no. 2-3 (1993): 273-8.

$\mathrm{TiO}_{2}$, engineering, reactor, aqueous phase, process efficiency.

421. Selzer, Volker H., and John C. Crittenden. Technological and Economic Feasibility of Full Scale Photocatalytic Oxidation Processes Applied to Air and Water Treatment, Michigan Technological University, Houghton, MI, 1995. National Center for Clean Industrial and Treatment Technologies, Michigan Technological University, Houghton, MI 49931.

$\mathrm{TiO}_{2}$, engineering, reactor, modeling, solar, metallized $\mathrm{TiO}_{2}$, gas phase, aqueous phase, modified $\mathrm{TiO}_{2}$, application, process efficiency, mechanism, immobilized $\mathrm{TiO}_{2}$, cost, catalyst reactivation.

422. Serpone, N., D. Lawless, R Terzian, C. Minero, and E. Pelizzetti. "Heterogeneous Photocatalysis: Photochemical Conversion of Inorganic Substances in the Environment: Hydrogen Sulfide, Cyanide, and Metals." Photochemical Conversion and Storage of Solar Energy, Proceedings of the 8th International Conference on Photochemical Conversion and Storage of Solar Energy, eds. E. Pelizzetti and M. Schiavello, 45 1-75, Dordrecht: Kluwer Academic Publishers, 1991.

$\mathrm{TiO}_{2}$, other semiconductor, reductant, metallized $\mathrm{TiO}_{2}$, aqueous phase, application, adsorption.

423. Serpone, N., P. Maruthamuthu, P. Pichat, E. Pelizzetti, and H. Hidaka "Exploiting the Interparticle Electron Transfer Process in the Photocatalyzed Oxidation of Phenol, 2-Chlorophenol and Pentachlorophenol: Chemical Evidence for Electron and Hole Transfer Between Coupled Semiconductors." J. Photochem. Photobiol., A 85, no. 3 (1995): 247-55. $\mathrm{TiO}_{2}$, other semiconductor, aqueous phase, modified $\mathrm{TiO}_{2}$, mechanism. 
424. Serpone, Nick. "A Decade of Heterogeneous Photocatalysis in Our Laboratory: Pure and Applied Studies in Energy Production and Environmental Detoxification." Res. Chem. Intermed.20, no. 9 (1994): 953-2.

$\mathrm{TiO}_{2}$, other semiconductor, reactor, solar, reductant, aqueous phase, application, process efficiency, mechanism, immobilized $\mathrm{TiO}_{2}$, metal removal.

425. Serpone, Nick. "Heterogeneous Photocatalysis for Environmental Water Purification: Why, What, and How?" Proc. Electrochem Soc.94- 19 (1994): 236-54.

$\mathrm{TiO}_{2}$, other semiconductor, solar, aqueous phase, application, mechanism.

426. Serpone, Nick, Darren Lawless, Rita Terzian, and Dan Meisel. "Redox Mechanisms in Heterogeneous Photocatalysis. The Case of Holes Versus Hydroxyl Radical Oxidation and Free Versus Surface Bound Hydroxyl Radical Oxidation Processes."Electrochem. Colloids Dispersions, [Symp. Electrochem. Microheterog. Fluids/Raymond A., and John Texter, 399-416, New York, NY: Mackay VCH, 1992.

$\mathrm{TiO}_{2}$, other semiconductor, oxidant, aqueous phase, mechanism.

427. Serpone, Nick, Ezio Pelizzetti, and Hisao Hidaka. "Identifying Primary Events and the Nature of Intermediates Formed During the Photocatalyzed Oxidation of Organics Mediated by Irradiated Semiconductors." Photocatalytic Purification and Treatment of Water and Air, eds. David F. Ollis and Hussain Al-Ekabi, 225-50, Trace Metals and the Environment, ed. Jerome 0. Nriagu, New York, NY: Elsevier, 1994.

$\mathrm{TiO}_{2}$, aqueous phase, mechanism.

428. Serpone, Nick, Rita Terzian, Darren Lawless, Pierre Kennepohl, and Genevieve Sauve. "On the Usage of Turnover Numbers and Quantum Yields in Heterogeneous Photocatalysis." $J$. Photochem. Photobiol., A 73, no. 1 (1993): 11-16.

$\mathrm{TiO}_{2}$, aqueous phase, process efficiency.

429. Serpone, Nick, Rita Terzian, Claudio Minero, and Ezio Pelizzetti. "Heterogeneous Photocatalyzed Oxidation of Phenol, Cresols, and Fluorophenols in Titanium Dioxide Aqueous Suspensions." Photosensitive Metal-Organic Systems, 28 1-3 14. Adv. Chem. Ser., eds. Charles Kutal and Nick Serpone. Washington, DC: American Chemical Society, 1993.

$\mathrm{TiO}_{2}$, aqueous phase, mechanism.

430. Serra, Francesca, Maria Trillas, Josep Garcia' and Xavier Domenech. "Titanium Dioxide-Photocatalyzed Oxidation of 2,4-Dichlorophenol." J. Environ. Sci. Health, Part A A29, no. 7 (1994): 1409-21.

$\mathrm{TiO}_{2}$, aqueous phase, adsorption.

431. Sharma, S. K., D. Yogi Goswami, and C. K. Jotshi. "Techno-Economic Analysis of Solar Detoxification Systems." Solar Engineering 1995, eds. William B. Stine, Tadayoshi Tanaka' and David E. Claridge, 467-73, New York, NY: ASME, 1995.

$\mathrm{TiO}_{2}$, engineering, reactor, modeling, aqueous phase, cost.

432. Sherrard, Kim B., Philip J. Marriott, Malcolm J. McCormick, Ray Colton, and Geoff Smith. "Electrospray Mass Spectrometric Analysis and Photocatalytic Degradation of Polyethoxylate Surfactants Used in Wool Scouring." Anal. Chem. 66, no. 20 (1994): 3394-9. $\mathrm{TiO}_{2}$, aqueous phase, application. 
433. Shiragami, Tsutomu, Shinako Fukami, Yuji Wada, and Shozo Yanagida. "Semiconductor Photocatalysis: Effect of Light Intensity on Nanoscale CdS-Catalyzed Photolysis of Organic Substrates." J. Phys. Chem. 97, no. 49 (1993): 12882-7.

other semiconductor, reductant, nonaqueous.

434. Sierka, R. A., and C. W. Bryant "Enhancement of Biotreatment Effluent Quality by Illuminated Titanium Dioxide and Membrane Pretreatment of the Kraft Extraction Waste Stream and by Increased Chlorine Dioxide Substitution." Water Sci Technol. 29, no. 5-6 (1994): 209-18. $\mathrm{TiO}_{2}$, aqueous phase, application, engineering.

435. Sierka, Raymond A., and Curtis W. Bryant. "Biological Treatment of Kraft Wastewater Following Pretreatment of the Extraction Waste Stream by Illuminating Titanium Dioxide and Membranes." Photocatalytic Purification and Treatment of Waer and Air, eds. David F. Ollis and Hussain Al-Ekabi, 275-90, Trace Metals and the Environment, ed. Jerome 0. Nriagu, New York, NY: Elsevier, 1994.

$\mathrm{TiO}_{2}$, engineering, aqueous phase, application.

436. Sirota, Vitaly. "Estimation of the Efficiency of Atmospheric Ozone Aerosol Sink." Proc. SPIE-Int. Soc. Opt. Eng. 2047, no. Atmospheric Ozone (1993): 216-18.

$\mathrm{TiO}_{2}$, other semiconductor, oxidant' gas phase, mechanism.

437. Sjogren, Jon C., and Raymond A. Sierka "Inactivation of Phage MS2 by Iron-Aided Titanium Dioxide Photocatalysis." Appl. Environ. Microbiol. 60, no. 1 (1994): 344-7.

Microbial, $\mathrm{TiO}_{2}$, disinfection, aqueous phase, modified $\mathrm{TiO}_{2}$.

438. Solymosi, Frigyes, and Imre Tombacz. "Photocatalytic Reaction of $\mathbf{H}_{2} \mathrm{O}+\mathrm{CO}$, Over Pure and Doped Rh/TiO,." Catal. Lett. 27, no. 1-2 (1994): 61-5.

$\mathrm{TiO}_{2}$, reductant, metallized $\mathrm{TiO}_{2}$, aqueous phase.

439. Somasundaram, Narayanan, Kasi Pitchumani, and Chockalingam Srinivasan. "Photoinduced Reduction of Aryl Methyl Sulfones on Titanium Dioxide." J. Chem. Soc., Chem. Commun., no. 12 (1994): 1473-4.

$\mathrm{TiO}_{2}$, reductant, nonaqueous.

440. Sopyan, Iis, Sadao Murasawa, Kazuhito Hashimoto, and Akira Fujushima. "Highly Efficient $\mathrm{TiO}_{2}$ Film Photocatalyst. Degradation of Gaseous Acetaldehyde." Chem. Lett., no. 4 (1994): 723-6.

$\mathrm{TiO}_{2}$, gas phase, modified $\mathrm{TiO}_{2}$.

441. Soria, J., M. J. Lopez-Munoz, V. Augugliaro, and J. C. Conesa. "Electron Spin Resonance Study of Radicals Formed During Photooxidation of Phenol on Titania" Colloids Surf., A 78, no. 1-3 (1993): 73-83.

$\mathrm{TiO}_{2}$, gas phase, mechanism, immobilized $\mathrm{TiO}_{2}$.

442. Spacek, W., R. Bauer, and G. Heisler. "Heterogeneous and Homogeneous Wastewater Treatment-Comparison Between Photodegradation With $\mathrm{TiO}_{2}$ and the Photo-Fenton Reaction." Chemosphere 30, no. 3 (1995): 477-84.

$\mathrm{TiO}_{2}$, aqueous phase, mechanism. 
443. Stafford, Ulick, Kimberly A. Gray, and Prashant V. Kamat. "Radiolytic and $\mathrm{TiO}_{2}$-Assisted Photocatalytic Degradation of 4-Chlorophenol. A Comparative Study." J.Phys. Chem. 98, no. 25 (1994): 6343-51.

$\mathrm{TiO}_{2}$, aqueous phase, mechanism.

444. Stafford, Ulick, Kimberly A. Gray, Prashant V. Kamat, and Arvind Varma. "An in Situ Diffuse Reflectance FTIR Investigation of Photocatalytic Degradation of 4-Chlorophenol on a $\mathrm{TiO}_{2}$ Powder Surface." Chem. Phys. Lett. 205, no. 1 (1993): 55-61.

$\mathrm{TiO}_{2}$, gas phase, mechanism.

445. Stradella, Luigi. "Photochemical Reactions of Halogenated Hydrocarbons on Pure and Doped Titanium Dioxide." Chemosphere 27, no. 7 (1993): 1129-36.

$\mathrm{TiO}_{2}$, gas phase, mechanism.

446. Sugimura, Hiroyuki, Tatsuya Uchida, Nobuo Shimo, Noboru Kitamura, and Hiroshi Masuhara "Scanning Tunneling Microscopy,Spectroscopy of Surface Oxide on Titanium: Photoexcitation Effect and Nanoanodization." Mol. Cryst. Liq. Cryst. Sci. Technol., Sect. A 252-253 (1994): 497-506. $\mathrm{TiO}_{2}$, modified $\mathrm{TiO}_{2}$.

447. Sullivan, Jack M., John H. Grinstead, Jr., Douglas J. Kiserow, Kathleen C. Pugh, and Joe Gautney. "TiO 2 Catalyzed Photo Oxidation of Atrazine in Dilute Aqueous Solutions Under Solar Irradiation: Process Development. " Solar Engineering 1994, eds. David E. Klett, Roy E. Hogan, and Tadayoshi Tanaka' 131-8, 1994.

$\mathrm{TiO}_{2}$, reactor, solar, pesticide, aqueous phase, immobilized $\mathrm{TiO}_{2}$, application.

448. Suri, Rominder P. S., Junbiao Liu, David W. Hand, John C. Crittenden, David L. Perram, and Michael E. Mullins. "Heterogeneous Photocatalytic Oxidation of Hazardous Organic Contaminants in Water." Water Environ. Res. 65, no. 5 (1993): 665-73.

$\mathrm{TiO}_{2}$, other semiconductor, metallized $\mathrm{TiO}_{2}$, aqueous phase, modified $\mathrm{TiO}_{2}$.

449. Sychev, M., N. Kostoglod, A. Kosorukov, V. Goncharuk, and V. Kashkovski. "Pillared Clays: Preparation and Investigation of (Physicochemical) and Catalytic Properties."Proc. - Pol.-Ger. Zeolite Colloq., ed. Michal Rozwadowski, 63-78, Torun, Pol.: Publisher: Nicholas Copernicus Univ. Press, 1992.

$\mathrm{TiO}_{2}$, aqueous phase, modified $\mathrm{TiO}_{2}$, immobilized $\mathrm{TiO}_{2}$.

450. T-Raissi, A., and N. Z. Muradov. "Flow Reactor Studies of Titanium Dioxide Photocatalytic Treatment of Airborne Nitroglycerin." Photocatalytic Purification and Treatment of Water and Air, eds. David F. Ollis and Hussain Al-Ekabi, 435-54, Trace Metals and the Environment, ed. Jerome 0. Nriagu, New York, NY: Elsevier, 1994.

$\mathrm{TiO}_{2}$, gas phase, application, oxidant.

451. Tahara, Shu, and Youzo Morita, inventors. "Method and Apparatus for Analyzing Nitrogen Compounds and Phosphorus Compounds in Water." Japan Shimadzu Corp., assignee. Europe Patent, 634646 Al. 18 January 1995. CA122:273701. $\mathrm{TiO}_{2}$, aqueous phase, application. 
452. Takahama, Koichi, Toshiharu Sako, Masaru Yokoyama, and Shozo Hirao. "Preparation of Platinum-Loaded Supercritically-Dried $\mathrm{TiO}_{2}$ Pillared Clays and Their Photocatalytic Activities in Oxidation of Carbon Monoxide." Nippon Kagaku Kaishi 7 (1994): 613-18.

$\mathrm{TiO}_{2}$, metallized $\mathrm{TiO}_{2}$, gas phase, modified $\mathrm{TiO}_{2}$, immobilized $\mathrm{TiO}_{2}$, adsorbent, adsorption, carbon monoxide.

453. Takeuchi, Koji, Kosuke Toyose, Shuzo Kutsuna, and Takashi Ibusuki. "Removal of Nitrogen Oxides From Air by Catalytic Oxidation With Photoenergy." Shigen to Kankyo 3, no. 2 (1994): 103- 10.

$\mathrm{TiO}_{2}$, gas phase, modified $\mathrm{TiO}_{2}$, application, immobilized $\mathrm{TiO}_{2}$, adsorbent.

454. Takiyama, Marcia M. K., Luis R. Takiyama, C. P. Huang, and Harm S. Huang. "Recovery of $\mathrm{Fe}$ (II)-EDTA From Wet-Scrubber Liquid Using $\mathrm{TiO}_{2}$,UV System." Hazard. Ind. Wastes 26 (1994): 595-60 1.

$\mathrm{TiO}_{2}$, reductant, oxidant, aqueous phase, application.

455. Tanaka' K., and T. Hisanaga "Photodegradation of Chlorofluorocarbon Alternatives on Metal Oxide." Sol. Energy 52, no. 5 (1994): 447-50.

$\mathrm{TiO}_{2}$, other semiconductor, gas phase, mechanism.

456. Tanaka' Keiichi, and Teruaki Hisanaga, inventors. "Photocatalytic Wastewater Treatment Apparatus.” Japan Kogyo Gijutsuin, assignee. Japan Patent, 06328068 A2.29 November 1994.

CA122:196089.

$\mathrm{TiO}_{2}$, other semiconductor, reactor, aqueous phase, application.

457. Tanaka' Shuzo, and Uttam K. Saha. "Effect of pH on Photocatalytic Degradation of Trichlorophenol Using Titanium Dioxide." Yosui to Haisui 36, no. 10 (1994): 883-6.

$\mathrm{TiO}_{2}$, aqueous phase, anion inhibition.

458. Tanaka' Shuzo, and Uttam Kumar Saha. "Effects of pH on Photocatalysis of 2,4,6-Trichlorophenol in Aqueous $\mathrm{TiO}_{2}$ Suspensions." Water Sci Technol. 30, no. 9 (1994): 47-57. $\mathrm{TiO}_{2}$, aqueous phase, anion inhibition.

459. Tang, Xiangping, Weichuan Jiang, Hong $\mathrm{Xu}$, and Hongjun Wang. "Study on Photodegradation of Printing and Dyeing Wastewater by Silver-Containing $\mathrm{TiO}_{2}$ Semiconductor Catalyst." Huanjing Wuran Yu Fangzhi 16, no. 5 (1994): 5-7, 21.

$\mathrm{TiO}_{2}$, metallized $\mathrm{TiO}_{2}$, aqueous phase, application.

460. Taqui Khan, M. M., D. Chatterjee, A. Hussain, and M. A. Moiz. "Synthesis and Characteristics of Mixed Ligand $\mathbf{R u}(\mathrm{III})$ Complexes With EDTA-Polypyridyl, and $\mathrm{Pt} / \mathrm{TiO}_{2} / \mathrm{RuO}_{2}$ Semiconductor Particulate System Modified by the Complexes." J. Photochem. Photobiol., A 76, no. 1-2 (1993): 97-101.

$\mathrm{TiO}_{2}$, reductant, aqueous phase, modified $\mathrm{TiO}_{2}$.

461. Tennakone, K., 0. A. Ileperuma, C. T. K. Thaminimulla, and J. M. S. Bandara

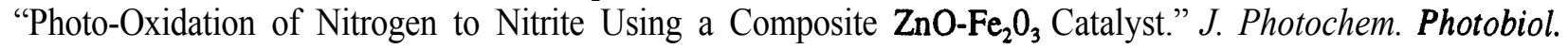
A: Chem. 66 (1992): 375-8.

other semiconductor. 
462. Tennakone, K., and U. S. Ketipearachchi. "Photocatalytic Method for Removal of Mercury From Contaminated Water." Appl. Catal., B 5, no. 4 (1995): 343-9.

$\mathrm{TiO}_{2}$, reductant, aqueous phase, application, metal removal.

463. Tennakone, K., S. Senadeera, and A. Priyadharshana "Titania Catalyzed Photooxidation of Water in the Presence of Methylene Blue." Sol. Energy Mater. Sol. Cells 29, no. 2 (1993): 109-3. $\mathrm{TiO}_{2}$, reductant, aqueous phase, modified $\mathrm{TiO}_{2}$.

464. Terzian, R. "Fundamental and Applied Studies in Heterogeneous Photocatalysis. Primary Radical Intermediates and Kinetics of Photo-Oxidation of Creosote Phenolics." Ph.D. diss., Concordia University, 1993.

aqueous phase, application, $\mathrm{TiO}_{2}$.

465. Tinucci, L., E. Borgarello, C. Minero, and E. Pelizzetti. "Treatment of Industrial Wastewaters by Photocatalytic Oxidation on Titanium Dioxide." Photocatalytic Purification and Treatment of Water and Air, eds. David F. Ollis and Hussain Al-Ekabi, 585-94, Trace Metals and the Environment' ed. Jerome 0. Nriagu, New York, NY: Elsevier, 1994.

$\mathrm{TiO}_{2}$, aqueous phase, application, reactor.

466. Tomkiewicz, M., Geula Dagan, and Zhu Zhu. "Morphology and Photocatalytic Activity of $\mathrm{TiO}_{2}$ Aerogels." Res. Chem. Intermed. 20, no. 7 (1994): 701-10.

$\mathrm{TiO}_{2}$, modeling, aqueous phase, modified $\mathrm{TiO}_{2}$, process efficiency, aerogel.

467. Trillas, Maria' Jose Peral, and Xavier Domenech. "Redox Photodegradation of 2,4-Dichlorophenoxyacetic Acid Over TiO. ." Appl. Catal., B 5, no. 4 (1995): 377-87.

$\mathrm{TiO}_{2}$, metallized $\mathrm{TiO}_{2}$, pesticide, aqueous phase.

468. Trillas, Maria' Jose Peral, Xavier Domenech, and Jaime Gimenez-Farreras. "Photocatalytic Oxidation of Phenol and 2,4-Dichlorophenol Over TiO ${ }_{2}$." Solar Engineering 1994, eds. David E. Klett, Roy E. Hogan, and Tadayoshi Tanaka' 171-4, 1994.

$\mathrm{TiO}_{2}$, aqueous phase.

469. Tseng, Jesseming. "Photocatalytic Oxidation of Phenols by Titanium Dioxide Suspension.” Ph.D. diss., Univ. Delaware, 1991.

$\mathrm{TiO}_{2}$, aqueous phase, mechanism.

470. Turchi, Craig S., James F. Klausner, D Yogi Goswami, and Edward Marchand. Field Test Results for the Solar Photocatalytic Detoxification of Fuel- Contaminated Groundwater,

NREL,TP-471-5345. NREL, Golden, CO, 1993.

aqueous phase, $\mathrm{TiO}_{2}$, engineering, process efficiency, reactor, solar.

471. Turchi, Craig S., and Mark S. Mehos. "Solar Photocatalytic Detoxification of Groundwater: Developments in Reactor Design." Chem. Oxid. Volume Date 1992, no. 2 (1994): 301-14. $\mathrm{TiO}_{2}$, engineering, reactor, solar, aqueous phase, immobilized $\mathrm{TiO}_{2}$.

472. Turchi, Craig S., Edward J. Wolfrum, and Richard A. Miller. Gas-Phase Photocatalytic Oxidation: Cost Comparison With Other Air Pollution Control Technologies, NREL,TP-47 1-7014. NREL, Golden, CO, 1994. DE95000241.

$\mathrm{TiO}_{2}$, engineering, reactor, gas phase, application, cost, competing technologies. 
473. Uchida, Hiroyuki, Shigeyoshi Itoh, and Hiroshi Yoneyama "Photocatalytic Decomposition of Propyzamide Using $\mathrm{TiO}_{2}$ Supported on Activated Carbon.” Chem. Lett., no. 12 (1993): 1995-8.

$\mathrm{TiO}_{2}$, pesticide, aqueous phase, immobilized $\mathrm{TiO}_{2}$, adsorbent.

474. Uchida, Hiroyuki, Shinobu Katoh, and Masahiro Watanabe. "Photocatalytic Decomposition of Trichlorobenzene Using $\mathrm{TiO}_{2}$ Supported on Nickel-Poly(Tetrafluoroethylene) Composite Plate." Chem. Lett., no. 4 (1995): 261-. $\mathrm{TiO}_{2}$, metallized $\mathrm{TiO}_{2}$, aqueous phase, immobilized $\mathrm{TiO}_{2}$.

475. Valladares, Julio E., and James R. Bolton. "A Method for the Determination of Quantum Yields in Heterogeneous Systems: the Titanium Dioxide Photocatalyzed Bleaching of Methylene Blue." Photocatalytic Purification and Treatment of Water and Air, eds. David F. Ollis and Hussain Al-Ekabi, 11 1-20, Trace Metals and the Environment, ed. Jerome 0. Nriagu, New York, NY: Elsevier, 1994.

$\mathrm{TiO}_{2}$, reactor, aqueous phase, process efficiency.

476. van Groenestijn, J. W., H. J. Doddema, H. J. G. Kok, and T. P. M. Koster. "Combined Photochemical and Biological Treatment of Off-Gases."Int. Symp. Environ. Biotechnol., Three-Day Symp., 2nd, 37-9, Rugby, UK: Inst. Chem. Eng., 1994.

$\mathrm{TiO}_{2}$, gas phase, biotreatment.

477. Vidal, A., J. Herrero, M. Romero, B. Sanchez, and M. Sanchez. "Heterogeneous Photocatalysis: Degradation of Ethylbenzene in $\mathrm{TiO}_{2}$ Aqueous Suspensions." J. Photochem. Photobiol., A 79, no. 3 (1994): 213-19.

$\mathrm{TiO}_{2}$, aqueous phase, mechanism.

478. Vidal, Alfonso, Manuel Romero, Benigno Sanchez, and Ferenc Mogyorodi. "Photocatalytic Degradation of Thiocarbamate Pesticides in Water. " Solar Engineering 1994, eds. David E. Klett, Roy E. Hogan, and Tadayoshi Tanaka' 117-21, 1994.

$\mathrm{TiO}_{2}$, pesticide, aqueous phase, application.

479. Vinodgopal, K. "Environmental Photochemistry: Electron Transfer From Excited Humic Acid to $\mathrm{TiO}_{2}$ Colloids and Semiconductor Mediated Reduction of Oxazine Dyes by Humic Acid." Res.

Chem. Intermed. 20, no. 8 (1994): 825-33.

nonaqueous, $\mathrm{TiO}_{2}$, reductant, aqueous phase, mechanism.

480. Vinodgopal K., Idriss Bedja, Surat Hotchandani, and Prashant V. Kamta. "A Photocatalyzed Approach for the Reductive Decolorization of Textile Azo Dyes in Colloidal Semiconductor Suspensions." Langmuir 10, no. 6 (1994): 1767-71.

$\mathrm{TiO}_{2}$, other semiconductor, reductant, aqueous phase, efficiency.

481. Vinodgopal, K., S. Hotchandani, and P. V. Kamat. "Electrochemically Assisted Photocatalytic Degredation of 4-Chlorophenol Using $\mathrm{TiO}_{2}$ Particulate Films." Symp. Environ. Aspects Electrochem. Photoelectrochem., 122-30, Proc.- Electrochem. Soc., no. 93-18. 1993. $\mathrm{TiO}_{2}$, reactor, aqueous phase, modified $\mathrm{TiO}_{2}$, process efficiency, immobilized $\mathrm{TiO}_{2}$.

482. Vinodgopal, K., and Prashant V. Kamat. "Enhanced Rates of Photocatalytic Degradation of an Azo Dye Using $\mathrm{SnO}_{2}$, TiO ${ }_{2}$ Coupled Semiconductor Thin Films." Environ. Sci. Technol. 29, no. 3 (1995): 841-5. $\mathrm{TiO}_{2}$, other semiconductor, aqueous phase. 
483. Vinodgopal, K., and Prashant V. Kamat. "Photochemistry of Textile Azo Dyes. Spectral Characterization of Excited State, Reduced and Oxidized Forms of Acid Orange 7." J. Photochem.

Photobiol., A 83, no. 2 (1994): 141-6.

$\mathrm{TiO}_{2}$, aqueous phase, mechanism, reductant.

484. Vinodgopal, K., Ulick Stafford, Kimberly A. Gray, and Prashant V. Kamat. "Semiconductor Particulate Films for the Photocatalytic Degradation of Organic Contaminants." Proc. Electrochem. Soc.94-19(Water Purification by Photocatalytic Photoelectrochemical, and Electrochemical Processes) (1994): 332-4 1.

$\mathrm{TiO}_{2}$, other semiconductor, aqueous phase, immobilized $\mathrm{TiO}_{2}$.

485. Vinodgopal, K., Ulick Stafford, Kimberly A. Gray, and Prashant V. Kamat.

"Electrochemically Assisted Photocatalysis. 2. The Role of Oxygen and Reaction Intermediates in the Degradation of 4-Chlorophenol on Immobilized $\mathrm{TiO}_{2}$ Particulate Films." J. Phys. Chem. 98, no. 27 (1994): 6797-803.

$\mathrm{TiO}_{2}$, aqueous phase, mechanism.

486. Viswanathan, B., U. D. Mary, and R. P. Viswanath. "Photocatalytic and Physicochemical Studies on Metalized Titania Systems." Stud. Surf. Sci. Catal. 75, New Frontiers in Catalysis, Pt. C (1993): 2147-50.

$\mathrm{TiO}_{2}$, metallized $\mathrm{TiO}_{2}$, gas phase, nonaqueous, mechanism.

487. Wada, Kenji, Kiyomi Yoshida, Tsuyoshi Takatani, and Yoshihisa Watanabe. "Selective Photooxidation of Light Alkanes Using Solid Metal Oxide Semiconductors." Appl.Catal., A 99, no. 1 (1993): 21-36.

$\mathrm{TiO}_{2}$, other semiconductor, gas phase, mechanism, process efficiency.

488. Wada, Yuji, Masahiko Taira, Dongyang Zheng, and Shozo Yanagida. "TiO ${ }_{2}$-Catalyzed Exhaustive Photooxidation of Organic Compounds in Perfluorotributylamine." New J. Chem. 18, no. 5 (1994): 589-96.

$\mathrm{TiO}_{2}$, nonaqueous, mechanism.

489. Wake, Hitoshi, and Tadashi Matsunaga, inventors. "Redox Reaction Using Photocatalysis of Semiconductor." Japan Pentel Kk, and Matsunaga Tadashi, assignees. Japan Patent, 06134476 A2. 17 May 1994. CA1 22:20314.

$\mathrm{TiO}_{2}$, other semiconductor, reactor, aqueous phase.

490. Waki, Kunio, Lingxuan Wang, Kayo Nohara, and Hisao Hidaka "Photocatalyzed Mineralization of Nitrogen-Containing Compounds At $\mathrm{TiO}_{2}, \mathrm{H}_{2} \mathrm{O}$ Interfaces." J. Mol. Catal. A: Chem. 95, no. 1 (1995): 53-9.

$\mathrm{TiO}_{2}$.

491. Wamhoff, Heinrich, Helmut Koch, Rolf Foerster, Christiane Herrmann, Sanaa m. S. Atta, M. Refat Mahran, and Mahmoud M. Sidky. "Photochemistry of Plant Protectants. On the Photodegradation of 1-(4-Chlorophenyl)-4,4-Dimethyl-3-( 1 H-1,2,4-Triazol- 1 -Ylmethyl)- Pentan-3-ol (Folicur)." Z. Naturforsch., B: Chem. Sci. 49, no. 2 (1994): 280-1. nonaqueous, $\mathrm{TiO}_{2}$, pesticide, reductant, aqueous phase, mechanism. 
492. Wang, Aihua, Jimmie G. Edwards, and Julian A. Davies. "Photooxidation of Aqueous Ammonia With Titania-Based Heterogeneous Catalysts.” Sol. Energy 52, no. 6 (1994): 459-66. $\mathrm{TiO}_{2}$, aqueous phase, modified $\mathrm{TiO}_{2}$.

493. Wang, FaYang, YuPan Weng, and LiangMu Lin. "Direct Observation of Crystal Surface Structure of Powdery Titanium Dioxide Photocatalyst by SAD in TEM." Photocatalytic Purification and Treatment of Water and Air, eds. David F. Ollis and Hussain AI-Ekabi, 753-8, Trace Metals and the Environment' ed. Jerome 0. Nriagu, New York, NY: Elsevier, 1994.

$\mathrm{TiO}_{2}$, mechanism.

494. Wang, Ko Ming, and Benito J. Marinas. "Control of VOC Emissions From Air-Stripping Towers: Development of Gas-Phase Photocatalytic Process." Photocatalytic Purification and Treatment of Water and Air, eds. David F. Ollis and Hussain Al-Ekabi, 733-9, Trace Metals and the Environment, ed. Jerome 0. Nriagu, New York, NY: Elsevier, 1994.

$\mathrm{TiO}_{2}$, gas phase, application.

495. Wang, Ko-ming, and Benito J. Marinas. "Gas-Phase Photocatalytic Process for the Control of VOC Emissions From Air-Stripping Towers."Proc. - Annu. Conf., Am. Water Works Assoc., 585-605, 1993.

$\mathrm{TiO}_{2}$, gas phase, immobilized $\mathrm{TiO}_{2}$.

496. Wang, Koming Vincent. "Gas Phase Photocatalytic Treatment of Trichloroethylene Using Titanium Dioxide Irradiated With Near Ultraviolet Light.” Ph.D. diss., Purdue Univ., West Lafayette, IN, USA, 1994.

$\mathrm{TiO}_{2}$, gas phase.

497. Wang, Kuo-Hua, Yung-Hsu Hsieh, and Kuo-Shu Huang. "Study on the Control of Aquatic Monochlorophenol Through Photocatalytic Decomposition." Zhongguo Huanjing Gongcheng Xuekan 4, no. 2 (1994): 111-8.

$\mathrm{TiO}_{2}$, aqueous phase.

498. Wang, Yuh-Yuan, and Chi-Chao Wan. "Investigation of Photoelectrochemical Reduction of Cupric Ions Over $\mathrm{TiO}_{2}$ in the Presence of Methanol." J. Photochem. Photobiol. A: Chem. 84 (1994): 195-202. $\mathrm{TiO}_{2}$, reductant, metallized $\mathrm{TiO}_{2}$, aqueousphase, mechanism.

499. Wang, Zeng-Hua, and Qi-Xing Zhuang. "Photocatalytic Reduction of Pollutant $\mathbf{H g}(\mathrm{II})$ on Doped WO, Dispersion." J. Photochem. Photobiol. A: Chem. 75 (1993): 105- 1.

other semiconductor, solar, reductant, aqueous phase.

500. Wang, Zhikai, and Charles Kutal. "Photocatalytic Mineralization of 2,4,6-Trinitrotoluene in Aqueous Suspensions of Titanium Dioxide." Chemosphere 30, no. 6 (1995): 1125-36.

$\mathrm{TiO}_{2}$, aqueous phase.

501. Ward, Michael D., James R. White, and Allen J. Bard. "Electrochemical Investigation of the Energetics of Particulate Titanium Dioxide Photocatalysts. The Methyl Viologen-Acetate System." $J$. Am. Chem. Soc. 105 (1983): 27-31.

$\mathrm{TiO}_{2}$, oxidant, aqueous phase, mechanism. 
502. Watabe, Tosha, and Eiichi Kojima, inventors. "Manufacture of Photocatalysts Containing Titanium Oxide and the Photocatalysts." Japan Toto Ltd, assignee. Japan Patent, 06205977 A2.26 July 1994. CA121:237634.

$\mathrm{TiO}_{2}$, gas phase, aqueous phase, modified $\mathrm{TiO}_{2}$, application, immobilized $\mathrm{TiO}_{2}$.

503. Watanabe, Toshiya, Atushi Kitamura, Eiichi Kojima, Kazuhito Hashimoto, and Akira Fujishima, inventors. "Air Treating Method Using Photocatalyst Under Interior Illumination." Japan, assignee. International Patent, 9411092 Al. 26 May 1994. CA121:140666.

$\mathrm{TiO}_{2}$, other semiconductor, disinfection, gas phase.

504. Watanabe, Toshiya, Atushi Kitamura, Eiichi Kojima, Chiaki Nakayama, Kazuhito Hashimoto, and Akira Fujishima "Photocatalytic Activity of Titanium Dioxide Thin Film Under Room Light." Photocatalytic Purification and Treatment of Water and Air, eds. David F. Ollis and Hussain Al-Ekabi, 747-5 1, Trace Metals and the Environment, ed. Jerome 0. Nriagu, New York, NY: Elsevier, 1994.

$\mathrm{TiO}_{2}$, gas phase, IAQ, immobilized $\mathrm{TiO}_{2}$.

505. Watts, Richard J., Sungho Kong, Margaret P. Orr, Glenn C. Miller, and Berch E. Henry. "Photocatalytic Inactivation of Coliform Bacteria and Viruses in Secondary Wastewater Effluent." Water Res. 29, no. 1 (1995): 95-100.

$\mathrm{TiO}_{2}$, disinfection, aqueous phase.

506. Watts, Richard J., Sungho Kong, Margaret P. Orr, and Glenn C. Miller. "Titanium Dioxide-Mediated Photocatalysis of a Biorefractory Chloroether in Secondary Wastewater Effluent." Environ. Technol. 15, no. 5 (1994): 469-75.

$\mathrm{TiO}_{2}$, aqueous phase, application.

507. Wei, Chang, Wen Yuan Lin, Zulkamain Zainal, Nathan E. Williams, Kai Zhu, Andrew P. Kruzic, Russell L. Smith, and Krishnan Rajeshwar. "Bactericidal Activity of $\mathbf{T i O}_{2}$ Photocatalyst in Aqueous Media: Toward a Solar-Assisted Water Disinfection System." Environ. Sci. Technol. 28, no. 5 (1994): 934-8.

$\mathrm{TiO}_{2}$, solar, disinfection.

508. Weng, Yupan, Fayang Wang, Liangmu Lin, and Ru Xie. "The Photocatalysis of Anatase Type Titanium Dioxide." Photocatalytic Purification and Treatment of Water and Air, eds. David F. Ollis and Hussain Al-Ekabi, 713-18, Trace Metals and the Environment, ed. Jerome 0. Nriagu, New York, NY: Elsevier, 1994.

$\mathrm{TiO}_{2}$, nonaqueous, mechanism.

509. Wilkins, Frank W., and Daniel M. Blake. "Use Solar Energy to Drive Chemical Processes." Chem. Eng. Prog. 90, no. 6 (1994): 41-9.

$\mathrm{TiO}_{2}$, engineering, reactor, solar, gas phase, aqueous phase, application.

510. Willner, I., Y. Eichen, and B. Willner. "Supramolecular Semiconductor Receptor Assemblies: Improved Electron Transfer At $\mathrm{TiO}_{2}$-Beta-Cyclodextrin Colloid Interfaces.” Res. Chem. Intermed. 20, no. 7 (1994): 681-700.

$\mathrm{TiO}_{2}$, metallized $\mathrm{TiO}_{2}$, aqueous phase, modified $\mathrm{TiO}_{2}$. 
511. Willner, Itamar, Yoav Eichen, Arthur J. Frank, and Marye Anne Fox. "Photoinduced Electron-Transfer Processes Using Organized Redox-Functionalized

Bipyridinium-Polyethylenimine-Titania Colloids and Particulate Assemblies." J. Phys. Chem. 97, no. 28 (1993): 7264-1.

$\mathrm{TiO}_{2}$, aqueous phase, modified $\mathrm{TiO}_{2}$.

512. Wyness, P., J. F. Klausner, D. Y. Goswami, and K. S. Schanze. "Performance of Nonconcentrating Solar Photocatalytic Oxidation Reactors, Part II: Shallow Pond Configuration.” J. Sol. Energy Eng. 116, no. 1 (1994): 8-13.

$\mathrm{TiO}_{2}$, reactor, solar, engineering, aqueous phase, immobilized $\mathrm{TiO}_{2}$.

513. Wyness, P., J. F. Klausner, D. Y. Goswami, and K. S. Schanze. "Performance of Nonconcentrating Solar Photocatalytic Oxidation Reactors, Part I: Flat-Plate Configuration." J. Sol. Energy Eng. 116, no. 1 (1994): 2-7.

$\mathrm{TiO}_{2}$, engineering, reactor, solar, aqueous phase, immobilized $\mathrm{TiO}_{2}$.

514. Wyness, P., James F. Klausner, D. Yogi Goswami, and Kirk S. Schanze. "Performance of Nonconcentrating Solar Photocatalytic Reactors: Part I - Flat Plate Configuration." Solar Engineering 1993, eds. Allan Kirkpatrick, and William Worek, 19-24, New York, NY: ASME, 1993.

aqueous phase, engineering, solar, $\mathrm{TiO}_{2}$.

515. Yamashita, H., H. Nishiguchi, N. Kamada, M. Anpo, Y. Teraoka, H. Hatano, S. Ehara, K. Kikui, L. Palmisano, and et al. "Photocatalytic Reduction of $\mathrm{CO}$, With $\mathrm{H}_{2} \mathrm{O}$ on $\mathrm{TiO}_{2}$ and $\mathrm{Cu} / \mathrm{TiO}_{2}$ Catalysts." Res. Chem. Intermed. 20, no. 8 (1994): 815-23.

$\mathrm{TiO}_{2}$, reductant, metallized $\mathrm{TiO}_{2}$, aqueous phase, modified $\mathrm{TiO}_{2}$.

516. Yamashita, Hiromi, Nobuhiro Kamada, Hong He, Kenichi Tanaka, Shaw Ehara, and Masakazu Anpo. "Reduction of $\mathrm{CO}$, With $\mathrm{H}_{2} \mathrm{O}$ on $\mathrm{TiO}_{2}(100)$ and $\mathrm{TiO}_{2}(110)$ Single Crystals Under W-Irradiation." Chem. Lett., no. 5 (1994): 855-58.

$\mathrm{TiO}_{2}$, gas phase, mechanism.

517. Yamazaki, Suzuko. "Destruction of Groundwater Contaminants Using Photocatalyst." Kagaku to Kogyo 47, no. 2 (Tokyo) (1994): 152-5.

$\mathrm{TiO}_{2}$, reactor, aqueous phase, modified $\mathrm{TiO}_{2}$, immobilized $\mathrm{TiO}_{2}$.

518. Yanai, Koichi, inventor. "Harmful Substance-Removing Agents, the Removal, and Apparatus.” Japan Nippon Zeon Co., assignee. Japan Patent' 06327965 A2.29 November 1994.

\section{CA122:141313.}

$\mathrm{TiO}_{2}$, other semiconductor, gas phase, modified $\mathrm{TiO}_{2}$, application, immobilized $\mathrm{TiO}_{2}$, adsorbent.

519. Yang, Junlin, Daohui Lu, and Guangzhi Xu. "Study of Free Radicals Generated in the Photoreaction of Co-Colloidal System.” Bopuxue Zazhi 11, no. 1 (1994): 107-1.

$\mathrm{TiO}_{2}$, other semiconductor, aqueous phase, modified $\mathrm{TiO}_{2}$, mechanism.

520. Yasumori, Atsuo, Koji Yamazaki, Shuichi Shibata, and Masayuki Yamane. "Preparation of $\mathrm{TiO}_{2}$ Fine Particles Supported on Silica Gel As Photocatalyst." J. Ceram. Soc. Japan 102, no. 8 (1994): 702-07.

$\mathrm{TiO}_{2}$, reductant, metallized $\mathrm{TiO}_{2}$, aqueous phase, modified $\mathrm{TiO}_{2}$, process efficiency, immobilized $\mathrm{TiO}_{2}$, mechanism. 
521. Yatmaz, H. C., C. R. Howarth, and C. Wallis. "Photocatalysis of Organic Effluents in a Falling Film Reactor." Photocatalytic Purification and Treatment of Water and Air, eds. David F. Ollis and Hussain Al-Ekabi, 795-800, Trace Metals and the Environment, ed. Jerome 0. Nriagu, New York, NY: Elsevier, 1994.

$\mathrm{TiO}_{2}$, reactor, aqueous phase, process efficiency.

522. Yoneyama, Hiroshi. "Effect of Supports for $\mathrm{TiO}_{2}$ Loading on the Rate of Photo-induced Oxidative Decomposition of Propionaldehyde." Presented at 17th U.S.-Japan Cooperative Seminar on Photoconversion and Photosynthesis Research, La Jolla' CA, 12-14 March 1995, 4 pp.

523. Yoshida, Katsuhiko, Kazuo Okamura, Koji Hirano, Kiyoshi Iguchi, Kiminori Itoh, and Masayuki Murabayashi. "Photocatalytic Degradation of Trichloroethylene in Water by Using Thin-Film $\mathrm{TiO}_{2}$ Prepared by Sol-Gel Process.” Mizu Kankyo Gakkaishi 17, no. 5 (1994): 324-9. $\mathrm{TiO}_{2}$, aqueous phase, immobilized $\mathrm{TiO}_{2}$.

524. Yost, Eric C., Isabel Tejedor-Tejedor, and Marc A. Anderson. "In Site CIR-FTIR Characterization of Salicylate Complexes At the Geothite,Aqueous Solution Interface." Environ. Sci. Technol. 24, no. 6 (1990): 822-28.

other semiconductor, aqueous phase, mechanism, surface binding.

525. You, Daoxin, Yongsheng Chen, and Shugui Dai. "Sunlight Photocatalytic Degradation of o-Chloroaniline." Water Qual. Res. J. Can. 30, no. 1 (1995): 61-7.

$\mathrm{TiO}_{2}$, other semiconductor, solar, pesticide, aqueous phase.

526. Yu, Ying, Boe Gu, Zhenxiao Wu, and Yajie Zhu. "Photo-Degradation of Organics in Wastewater With Catalysts of Titanium Dioxide." Shiyou Daxue Xuebao, Ziran Kexueban 18, no. 2 (1994): 90-5.

$\mathrm{TiO}_{2}$, aqueous phase, modified $\mathrm{TiO}_{2}$.

527. Yue, P. L., and D. Allen. "Photocatalytic Degradation of Atrazine." Photocatalytic Purification and Treatment of Water and Air, eds. David F. Ollis and Hussain Al-Ekabi, 607-1 1, Trace Metals and the Environment' ed. Jerome 0. Nriagu, New York, NY: Elsevier, 1994. $\mathrm{TiO}_{2}$, pesticide, aqueous phase, modified $\mathrm{TiO}_{2}$, mechanism.

528. Zhang, Manping, Shinan Xie, and Weiping Chen. "Study on the Isotherm for Adsorption of Dye on Titanium Dioxide." Haiyang Huaxue Lunwenxuan, ed. Manping Zhang, 41-4, Beijing, Peop. Rep. China: Haiyang Chubanshe, 1994.

$\mathrm{TiO}_{2}$, aqueous phase, adsorption.

529. Zhang, P., R. J. Scrudato, J. J. Pagano, and R. N. Roberts. "Photocatalytic Decomposition of PCBs in Aqueous Systems With Solar Light." Photocatalytic Purification and Treatment of Water and Air, eds. David F. Ollis and Hussain Al-Ekabi, 619-24, Trace Metals and the Environment, ed. Jerome 0. Nriagu, New York, NY: Elsevier, 1994.

$\mathrm{TiO}_{2}$, aqueous phase, application.

530. Zhang, Yin, J. C. Crittenden, D. W. Hand, D. L. Perram, and E. G. Marchand. "Solar Detoxification of Groundwater Using Fixed-Bed Photocatalysis." The Proceedings of the Water Environment Federation 67th Annual Conference, 659-70, 1994.

$\mathrm{TiO}_{2}$, engineering, reactor, solar, metallized $\mathrm{TiO}_{2}$, aqueous phase, application, immobilized $\mathrm{TiO}_{2}$. 
531. Zhang, Yin, John C. Crittenden, and David W. Hand. "The Solar Photocatalytic Decontamination of Water." Chem. Ind September (1994): 714-17.

$\mathrm{TiO}_{2}$, other semiconductor, engineering, reactor, solar, aqueous phase, modified $\mathrm{TiO}_{2}$, application, cost.

532. Zhang, Yin, John C. Crittenden, David W. Hand, and David L. Perram. "Fixed-Bed

Photocatalysts for Solar Decontamination of Water." Environ. Sci. Technol. 28, no. 3 (1994): 435-2.

$\mathrm{TiO}_{2}$, aqueous phase, modified $\mathrm{TiO}_{2}$, immobilized $\mathrm{TiO}_{2}$, solar, process efficiency.

533. Zhao, Jincai, Hisao Hidaka, Akira Takamura, Ezio Pelizzetti, and Nick Serpone.

"Photodegradation of Surfactants. 11. Zeta-Potential Measurements in the Photocatalytic Oxidation of Surfactants in Aqueous Titania Dispersions." Langmuir 9, no. 7 (1993): 1646-50.

$\mathrm{TiO}_{2}$, aqueous phase, mechanism, adsorption.

534. Zhou, Yongqiu, Yunzhi Cai, and Yiyi Bau. "Study on the Photocatalytic Degradation of Trichloroacetic Aldehyde Solution Containing Semiconductor Compound." Huanjing Wuran Yu Fangzhi 16, no. 1 (1994): 15-17.

$\mathrm{TiO}_{2}$, aqueous phase. 


\subsection{Supplemental References}

535. Augustynski, Jan. "Aspects of Photo-Electrochemical and Surface Behavior of Titanium(IV) Oxide." 61 pp. Structure and Bonding, 69. Berlin, Germany: Springer Verlag, 1988. adsorption/ aqueous phase/ catalyst characterization/ immobilized $\mathrm{TiO}_{2} /$ process efficiency/ mechanism/ $\mathrm{TiO}_{2}$.

536. Barbeni, Massimo, Edmondo Pramauro, Ezio Pelizzetti, Enrico Borgarello, Nick Serpone, and Mary A. Jamieson. "Photochemical Degradation of Chlorinated Dioxins, Biphenyls, Phenols and Benzene on Semiconductor Dispersions." Chemosphere 15, no. 9-12 (1986): 1913-6. aqueous phase.

537. Bickley, R. I. "Fundamental Aspects of the Adsorption and the Desorption of Gases At Solid Surfaces Under Illumination." NATO ASI Ser., Ser. C 146 (1985): 379-88. adsorption/ catalyst characterization/ photocatalysis.

538. Borgarello, Enrico, Nick Serpone, Michael Gratzel, and Ezio Pelizzetti. "Photodecomposition of Hydrogen Sulfide in Aqueous Alkaline Media Catalyzed by Ruthenium Dioxide-Loaded Alumina in the Presence of Cadmium Sulfide. Application of the Inter-Particle Electron Transfer Mechanism.” Inorg. Chim. Acta 112, no. 2 (1986): 197-201. aqueous phase/ other semiconductor.

539. Borgarello, Enrico, Nick Serpone, Paul Liska, Wilson Erbs, Michael Gratzel, and Ezio Pelizzetti. "Photocleavage of Hydrogen Sulfide in Alkaline Aqueous Media With a Ruthenium Dioxide-Loaded Cadmium Sulfide Catalyst Supported on a Polycarbonate Matrix." Gazz. Chim. Ital. 115, no. 11-12, Pt. A (1985): 599-602. aqueous phase/ other semiconductor.

540. Borgarello, Enrico, Rita Terzian, Nick Serpone, Ezio Pelizzetti, and Massimo Barbeni. "Photocatalyzed Transformation of Cyanide to Thiocyanate by Rhodium-Loaded Cadmium Sulfide in Alkaline Aqueous Sulfide Media." Inorg. Chem. 25, no. 13 (1986): 2135-7. aqueous phase/ other semiconductor.

541. Chen, Dezhi, and Xuezhu Wen. "Oxidation of Sulfite With the Heterogeneous Tungsten Trioxide/.Alpha.-Ferric Oxide/Tungsten Photocatalyst in Aqueous Solution." Huaxue Tongbao, no. 5 (1988): 32-3.

aqueous phase/ other semiconductor.

542. Chen, Dezhi, Xuezhu Wen, and Wennu Jin. "Study of Oxidation of Iodide on a Heterogeneous Photocatalyst Tungsten Trioxide/.Alpha-Ferric Oxide/Tungsten in Aqueous Solution." Huaxue Tongbao, no. 2 (1989): 32-4.

aqueous phase/ other semiconductor.

543. Childs, Lorette Pruden, and David F. Ollis. "Is Photocatalysis Catalytic?" J. Catal. 66, no. 2 (1980): 383-90. aqueous phase/ mechanism.

544. Daroux, M., Y. Parent' and D. Klavana. "A New Reactor for the Study of Photocatalytic Reactions." Chem. Eng. Commun. 4 (1980): 501-6. gas phase/ reactor / engineering. 
545. Domenech, J., and J. Peral, Cerdanyola, Spain. "Removal of Toxic Cyanide From Water by Heterogeneous Photocatalytic Oxidation Over Zinc Oxide.” Sol. Energy 41, no. 1 (1988): 55-9. aqueous phase/ other semiconductor.

546. Fox, Marye Anne, Austin, TX 78712, USA. "Organic Heterogeneous Photocatalysis: Chemical Conversions Sensitized by Irradiated Semiconductors." Acc. Chem Res. 16, no. 9 (1983): $314-21$. aqueous phase/ mechanism/ nonaqueous.

547. Henglein, Arnim. "Small-Particle Research: Physicochemical Properties of Extremely Small Colloidal Metal and Semiconductor Particles." Chem. Rev. 89, no. 8 (1989): 1861-73. catalyst characterization/ modified $\mathrm{TiO}_{2} /$ other semiconductor.

548. Hodes, Gary, and M. Gratzel. "Photoelectrochemistry At Semiconductor Electrodes and Small Particles: A Comparative Study." Nouveau J. De Chemie 8 (1984): 509-20. photocatalyst/ mechanism/ process efficiency.

549. Kalyanasundaram, K. "Semiconductor Particulate Systems for Photocatalysis and Photosynthesis: an Overview." Energy Resources Through Photochemistry and Catalysis, ed. Michael Gratzel, 217-60. New York: Academic Press, 1983. aqueous phase/ catalyst characterization/ mechanism/ metallized $\mathrm{TiO}_{2} /$ other semiconductor/ oxidant.

550. Kalyanasundaram, Kuppuswamy, and M. Graetzel. "Heterogeneous Photocatalysis With Semiconductor Particulate Systems.” Springer Ser. Chem. Phys 35 (1984): 11 1-39. mechanism.

551. Lewis, Nathan S. "Mechanistic Studies of Light-Induced Charge Separation At Semiconductor/Liquid Interfaces." Acc. Chem. Res. 23 (1990): 176-83.

mechanism/ nonaqueous/ aqueous phase.

552. Marinangeli, R. E., and D. F. Ollis. "Photo-Assisted Heterogeneous Catalysis With Optical Fibers. Part III. Photoelectrodes." AIChE J.28, no. 6 (1982): 945-55.

aqueous phase/ engineering/ reactor / immobilized $\mathrm{TiO}_{2}$.

553. Marinangeli, R. E., and D. F. Ollis. "Photo-Assisted Heterogeneous Catalysis With Optical Fibers. II. Nonisothermal Single Fiber and Fiber Bundle." AIChE J. 26, no. 6 (1980): 1000-8. aqueous phase/ engineering/ reactor / immobilized $\mathrm{TiO}_{2}$.

554. Marinangeli, Richard E., and David F. Ollis. "Photoassisted Heterogeneous Catalysis With Optical Fibers. I. Isolated Single Fiber." AIChE J. 23, no. 4 (1977): 415-26. aqueous phase/ immobilized $\mathrm{TiO}_{2} /$ engineering/ reactor.

555. Muto, H., M. Shinada, and Y. Takizawa. "Heterogeneous Photolysis of Polychlorinated Dibenzo-p-Dioxins on Fly Ash in Water-Acetonitrile Solution in Relation to the Reaction With Ozone." Environ. Sci. Technol. 25 (1991): 3 16-22.

Dibenzo-p-dioxins/ other semiconductor/ nonaqueous.

556. Ollis, David F. "Contaminant Degradation in Water." Environ. Sci. Technol. 19, no. 6 (1985): 480-84. aqueous phase. 
557. Ollis, David F. "Process Economics for Water Purification: a Comparative Assessment." NATO ASI Ser., Ser. C, Photocatal. Environ. 237 (1988): 663-77.

aqueous phase/ engineering/ cost.

558. Ollis, David F., Ezio Pelizzetti, and Nick Serpone. "Photocatalyzed Destruction of Water Contaminants.” Environ. Sci. Technol. 25, no. 9 (1991): 1522-29.

aqueous phase/ metal removal/ oxidant/ mechanism/ pesticide.

559. Ollis, David F., and Ann L. Pruden. "Photostimulated Heterogeneous Catalysis: Description of the Process; Oxidation of Hydrocarbon and Chlorohydrocarbon Compounds." Geterogen. Khimiya Atmosf., L (1987): 251-63.

aqueous phase.

560. Pelizzetti, E., E. Pramauro, C. Minero, N. Serpone, and E. Borgarello. "Photodegradation of Organic Pollutants in Aquatic Systems Catalyzed by Semiconductors." NATO ASI Ser., Ser. C,

Photocatal. Environ. 237 (1988): 469-97.

photocatalysis.

561. Pelizzetti, Ezio. "Homogeneous and Heterogeneous Photocatalysis." Chim. Ind., Milan 68, no. 10 (1986): 51-2.

aqueous phase/ photocatalysis.

562. Pelizzetti, Ezio, Massimo Barbeni, Edmondo Pramauro, Wilson Erbs, Enrico Borgarello, Mary A. Jamieson, and Nick Serpone. "Photocatalytic and Photosynthetic Processes With Semiconductor Particulates." Quim. Nova 8, no. 4 (1985): 288-302.

563. Pelizzetti, Ezio, Enrico Borgarello, and Nick Serpone. "Photocatalytic Reactions of Organic Compounds. Hydrogen Generation From Organics and Degradation of Wastes." NATO ASI Ser., Ser. C 146 (1985): 305-19.

aqueous phase/ mechanism.

564. Pelizzetti, Ezio, and Nick Serpone. "Homogeneous and Heterogeneous Photocatalysis. [Proceedings of the NATO Advanced Research Workshop on Homogeneous and Heterogeneous Photocatalysis, Maratea Potenza, Italy, September 1-7, 1985]." 721 pp. NATO Advanced Science Institutes Series C: Mathematical and Physical Sciences, 174. Dordrecht, Neth: D. Reidel Publishing Co., 1986.

photocatalysis.

565. Peral, J., and D. F. Ollis. "Heterogeneous Photocatalysis for Air Purification." 1991 Summer National Meeting of AIChE. gas phase/ immobilized $\mathrm{TiO}_{2}$.

566. Peral, Jose, Juan Casado, and Javier Domenech. "Light-Induced Oxidation of Phenol Over ZnO Powder." J. Photochem. and Photobiol., A: Chem. 44 (1988): 209-17. aqueous phase/ other semiconductor.

567. Sato, S., and J. M. White. "Photoassisted Catalysis Using Platinized Titania." Stud. Surf. Sci. Catal., Pt. B 7 (1981): 1500-1.

aqueous phase. 
568. Sato, Shinri. "Gas Phase Photoelectrochemical Reactions Over Metalized, Powdered Semiconductors.” Denki Kagaku Oyobi Kogyo Butsuri Kagaku 53, no. 1 (1985): 9-14.

aqueous phase/ metallized $\mathrm{TiO}_{2}$.

569. Scaife, D. E. "Oxide Semiconductors in Photoelectrochemical Conversion of Solar Energy." Solar Energy 25, no. 1 (1980): 41-54.

catalyst characterization/ other semiconductor/ $\mathrm{TiO}_{2} /$ modeling.

570. Serpone, Nick. "Solar Photochemistry and Heterogeneous Photocatalysis: a Convenient and Practical Utilization of Sunlight Photons." Photochem. Energy Convers., Proc. Int. Conf. Photochem. Convers. Storage Solar Energy, eds. James R. Norris, Jr. and Dan Meisel, 297-3 15, 1989. aqueous phase/ metal removal/ other semiconductor.

571. Serpone, Nick, Enrico Borgarello, and Ezio Pelizzetti. "Photoreduction and Photodegradation of Inorganic Pollutants: I. Cyanides.” NATO ASI Ser., Ser. C, Photocatal. Environ. 237 (1988): 499-526.

aqueous phase/ other semiconductor.

572. Turchi, Craig S., and David F. Ollis. "Photocatalytic Reactor Design: An Example of Mass-Trasfer Limitations With an Immobilized Catalyst." J. Phys. Chem. 92 (1988): 6852-53. engineering/ reactor / immobilized $\mathrm{TiO}_{2} /$ aqueous phase.

573. Vinodgopal, K., and P. V. Kamat. "Environmental Photochemistry on Surfaces. Charge Injection From Excited Fulvic Acid into Semiconductor Colloids." Environ. Sci. Technol. 26 (1992): 1963-66.

fulvic acid/ aqueous phase/ other semiconductor.

574. Watanabe, Tadashi, Takuo Takizawa, and Kenichi Honda. "Heterogeneous Photocatalytic Processes and Semiconductor Photoelectrode Reactions." Shokubai 20, no. 6 (1978): 370-80.

photocatalysis/ solar. 


\subsection{Distribution List}

Civil Engineering Research Foundation 1015 15th Street NW, Suite 600

Washington, DC 20005

Dr. Manuel Romero Alvarez

CIEMAT-IER

Avda. Complutense 22

28040 Madrid

SPAIN

Dr. James A. Armstrong

Manager of Air Quality Services

Foster Wheeler Environment Corporation 143 Union Blvd., Suite 1010

Lakewood, CO 802281824

Ms. Sayward Ayre

114 Pear Street NE, No. 2

Olympia, WA 98506

Dr. Ron Balkissoon, M.D.

Staff Physician

National Jewish Center for Immunology and Respiratory Medicine

1400 Jackson St.

Denver, CO 80206

Mr. Chuck Beck

Coors Brewing Co

Golden, CO 80401
Dr. Craig Adams

Clemson University

Environmental Systems Engineering

Clemson Research Park

Clemson, SC 296340919

Dr. Marc Anderson

University of Wisconsin

Water Chemistry Program

660 North Park Street

Madison, WI 53706

Ms. Karen Atkinson

WasteTech News

131 Madison Street

Denver, CO 80206

Mr. Mike Bahm

Senior Program Manager

Radian Corporation

1801 Broadway, Suite 1000

Denver, CO 80202

Dr. Alan Bard

University of Texas

Department of Chemistry

Austin, TX 78712-1167

Mr. Gino Beer

5 via Risorgimento

16033 Lavagna,

Genoa

ITALY

Ms. Kathleen Bennett

Vice President

James River Corporation

P.O. Box 2218

Richmond, VA 23217

Dr. Gale Biggs

W. Gale Biggs Associates

P.O. Box 3344

Boulder, CO 80303
Dr. Hussain Al-Ekabi

Hitech Advanced Photo-Oxidation

71 Brandy Lane Road

London, Ontario,

CANADA, N6G 4S2

Mr. R. D. Andrew

Mobil Research and Development Corp.

Engineering Department

P.O. Box 1026

Princeton, NJ 08543

Professor Jan Augustynski

Université de Genève

Chimie Appliquée

Sciences II

30 quai E. Ansermet, $\mathrm{CH}-1211$ Geneve 4

SWITZERLAND

Dr. Detlef Bahnemann

Institut fur Solarenergieforschung $\mathrm{GmbH}$

Sokelantstrasse 5

D-30165 Hannover

GERMANY

Mr. Herb Bassow

Germantown Friends School

31 West Coulter Street

Philadelphia, PA 19144

\section{Mr. Kelly Beninga}

Science Applications International Corp. 15000 W. 6th Ave., Suite 202

Golden, CO 80401

Dr. Elliot Berman

Resident

Photox Corporation

P. 0. Box 15717

Boston, MA 02215

Mr. Peter J. Blake

Dir. of Pub. And Env. Affairs

North East Fabricare Association

343 Salem Street

Wakefield, MA 01880

Box 4054

Richmond, CA 94804 
Dr. Julian Blanco

CIEMAT-PSA

Aptdo. 22

E-4200 Tabernas (Almeria)

SPAIN

Dr. Jim Bolton

Solarchem Environmental Systems

130 Royal Crest Court

Markham, Ontario

CANADA L3R OA1

Mr. Steven Bowles

Kleinfelder

3249 East Harbour Drive

Phoenix, AZ 850347227

Mr. Brian Butters

General Manager

Pulifics

161 Mallard Road

Hyde Park, Ontario NOM 120

CANADA

\section{Dr. Luis Canas}

Savanah River Westinghouse Co.

Mail Stop 244-2h

P.O. Box 616

Aiken, SC 29802

Mr. Clifton Carwile

U.S. Dept. of Energy

EE-222, 5G-035

Office of Industrial Technologies

1000 Independence Ave. SW

Washington, DC 20585

Dr. Eileen Chant

University of Turabo

School of Engineering

Apartado 3030

Gurabo, Puerto Rico 00778

\section{Mr. James D. Chrisman}

Vice President

Stapleton Redevelopment Foundation

730 17th Street, Suite 340

Denver, CO 80202
Ms. Cathy Blount

Chemical Engineering Department

University of Colorado

Campus Box 424

Boulder, CO 80309

Dr. Paul Bonczyk

UTRC - M.S. 30

Silver Lane

East Hartford, CT 06108

Mr. Mark Burger

U.S. Department of Energy

1 South Wacker Drive, Suite 2380

Chicago, IL 606064616

Mr. Beningo Cabrero

CIEMAT-IER

Avda. Complutense 22

28040 Madrid

SPAIN

Dr. Nelson Cardona

University of Puerto Rico at Mayaguez

Chemical Engineering Dept.

P.O. Box 5000

Mayaguez, PR 00681-5000

Dr. Stephen R. Cater

Manager, Research \& Process Engineering

Solarchem Environmental Systems

130 Royal Crest Court

Markham, Ontario LeR0A1

CANADA

Mr. James M. Chavez

Sandia National Laboratories

Solar Thermal Test

Department 6215, MS1 127

Albuquerque, NM 87185

Dr. Ronald Clazie

Vice President, Engineering

Free-Flow Packaging Corporation

1903 Charter Street

Redwood City, CA 94063
Mr. David Bluestein

Dow Environmental

49 Stevenson Street

Suite 600

San Francisco, CA 94105

Ms. La Ronda V. Bowen

Public Advisor

South Coast Air Quality Management

District

21865 Copley Drive

Diamond Bar, CA 91765

Mr. Heyward Burnette

LOGTEC, Incorporated

2900 Residential Drive

Fairborn, OH 45324

Mr. Mark Camenzind

Balazs Labs

252 Humboldt Court

Sunnyvale, CA 94089-13 15

Mr. Jeffrey Carmody

Santa Barbara Air Pollution Control

District

Innovative Technologies Group

26 Castilian Drive, Suite B-23

Goleta, CA 93117

Mr. Jerry Ceasar

BP

17618 Plum Creek Trail

Chagrin Falls, OH 44023

Mr. Daniel Chen

Lamar University

Department of Chemical Engineering

P.O. Box 10053

Beaumont, TX 77710

Dr. Armin Clobes

Senior Research Associate

S.C. Johnson \& Son, Inc.

1525 Howe Street

Racine, WI 53403-50 11 
Mr. James L. Condit

Project Manager

Kleinfelder

3249 East Harbour Drive

Phoenix, AZ 850347227

Mr. Gerald Cooper

Photocatalytics, Inc.

755 South 42nd Street

Boulder, CO 80303

Dr. John Crittenden

Michigan Technological University

Environmental Engineering Center

1400 Townsend Drive

Houghton, MI 49931

\section{Dr. Chuck Darvin}

U.S. EPA/AEERL

Research Triangle Park, NC 27711

Mr. Arthur Davidson

Davidson \& Associates Environmental 4814 Somerset Drive

Bellevue, Washington 98006

Ms. Cathy Dombrowski

Haz TECH News

14120 Huckleberry Lane

Silver Springs, MD 20906-2012

Mr. Roch Ducey

U.S. Army Corps of Engineers, CERL

P.O. Box 9005

Champaign, IL 61826

Dr. Gary Epling

University of Connecticut

Department of Chemistry

215 Glenbrook Road U-60

Storrs, CT 06269-3060
Mr. Richard Consolas

Editor

Environtech

P. 0. Box 640310

Oakland Gardens, NY 11364

Dr. John A. Cooper

TRC Environmental Corp.

12242 SW Garden Place

Tigard, OR 97223

Dr. Donald Cropek

USA CERL

P. 0. Box 9005

Champagne, IL 618269005

Dr. Abhaya Datye

University of New Mexico

Center for Microengineered Ceramics

Albuquerque, NM 87131

Dr. David Deberry

Corporate Fellow

Radian Corporation

8501 Mapac Blvd.

Austin, TX 78759

Dr. Xavier Domenech

Univ. Autonoma Barcelona

Dtp. Quimica, Bellaterra

08193 Barcelona

SPAIN

Mr. Mike Dunn

Air Manager

Naval Surface Warfare Center, Indian

Head

101 Strauss Ave.

Indian Head, MD 20640

Dr. John Falconer

University of Colorado

Department of Chemical Engineering

Campus Box 424

Boulder, CO 80309
Dr. Donald Conte

California University of Pennsylvania

Earth Science Box 55

California, PA 15419

Mr. Douglas Cregar

DuPont Chemicals R\&D

Jackson Laboratory

Deepwater, NJ 08023

Dr. John Daily

Department Chair

University of Colorado

Department of Mechanical Eng.

Engineering Center

Boulder, CO 80309

Mr. Roger Davenport

Science Applications International

Corporation

15000 W. 6th Ave., Suite 202

Golden, CO 80401

Dr. Barry Dellinger

UDRC

300 College Park

Dayton, OH 45469

Dr. Jose M. Doiia

Departamento de Química

Universidad de Las Palmas de Gran

Canaria

Campus Universitario de Tafira

35017 - Las Palmas

SPAIN

Dr. David Ensor

Research Triangle Institute

P. 0. Box 12194

Research Triangle Park, NC 27709-2194

Mr. William Fisher

International Fabricare Institute

12251 Tech Road

Silver Spring, MD 20904 
Dr. Renee Ford

Editor, Materials Technology Magazine P.O. Box 72

Harrison, NY 10528-0072

Dr. Mary Anne Fox

University of Texas

Department of Chemistry

Austin, TX 78712-1167

Mr. Norman Francinques

US Army Engineers

3909 Halls Ferry Rd.

Mail Code CEWES-EE-R

Vicksburg, MI 39180-6199

Mr. Ralph Froehlich

Resident

Helix Environmental, Inc.

7720 Paragon Road, Suite B

. Dayton, OH 45459

Mr. James Garmaker

Senior Project Engineer

3M Company

P. 0. Box 33331

Building 42-4E-03

St. Paul, MN 55 133-3331

Dr. Anne Giesecke

Vice President, Environmental Activities

American Bakers Association

1350 I Street NW, Suite 1290

Washington, DC 20005

Mr. Steven Gorman

American Energy Technologies, Inc P.O. Box 1865

Green Cove Springs, FL 32043
Dr. Fardad Forouzan

University of Texas

Department of Chemistry

Austin, TX 78712

Mr. Randy Fox

Kleinfelder

3249 East Harbour Drive

Phoenix, AZ 850347227

Mr. Allan L. Frank

Editor

The Solar Letter

9124 Bradford Rd.

Silver Springs, MD 20901-4918

Dr. Karl-Heinz Funken

German Aerospace Research Development

Energy Technology Division

D-5 1140 Koln

GERMANY

Mr. Gary Garner

3M Company

P. 0. Box 33331

Building 21-2W-05

St. Paul, MN 55133-3331

Dr. William Glaze

University of North Carolina

Environmental Science \& Engineering

Rosenau Hall, CB\# 7400

Chapel Hill, NC 27599-7400

Dr. Yogi Goswami

Professor of Mechanical Engineering

University of Florida

College of Engineering

220 MEB

Gainesville, FL 326 1 1-2050

Dr. Michael Grätzel

Institut de Chemic Physique

EPFL

1015 Lausanne

SWITZERLAND
Dr. Nancy Foster-Mills

New Mexico State University

2241 Entrada Del Sol \#1J

Las Cruces, NM 88001

Mr. Robert Fox

Vice President

IT corporation

312 Directors Drive

Knoxville, TN 37923

Dr. Jim Freihaut

United Technologies Research Center

411 Silver Lane, MS 129-24

East Hartford, CT 06108

Mr. David Gaboardi

Mark III Industries

P.O. Box 2525

Ocala, FL 34478-2525

Mr. Ranji George

South Coast Air Quality Management

District

21865 Copley Drive

Diamond Bar, CA 91765-4182

Mr. James Goodrich

Environmental Scientist

U.S. EPA

Drinking Water Research

26 W. Martin Luther King Dr

Cincinnati. Ohio 45268

Mr. John Graf

NASA JSC

Mail Code EC3

Houston, TX 77058

Dr. Kimberly Gray

Notre Dame University

Dept of Civil Engineering

Notre Dame, IN 46556 
Dr. Robert Hall

Sr. Research Scientist

UTRC - M.S. 30

Silver Lane

East Hartford, CT 06108

Mr. Bob Henderson

Matrix Photocatalytic, Inc.

22 Pegler Street

London, Ontario N5Z 2B5

CANADA

Dr. Michael Hoffmann

W. M. Keck Laboratories

California Institute of Technology

Pasadena, CA 91125

Mr. Robert Hulsey

Black \& Veatch

8400 Ward Parkway

Kansas City, MO 64114

Dr. John Ireland

U.S. EPA

Office of Research and Development

Risk Reduction Engineering Lab

26 W. Martin Luther King Dr.

Cincinnati, OH 45268

Ms. Sandra Jenkins

Parsons Engineering Science

Woodruff Annex Complex

P. 0. Box 1625

Idaho Falls, ID 83415-3954

Mr. Mike Kemme

U.S. Army Construction

Engineering Research Laboratories

P. 0. Box 9005

Champaign, IL 61826

\section{Dr. Jim Klausner}

University of Florida

College of Engineering

Dept. of Mech. Engineering

Gainesville, FL 32611
Dr. Adam Heller

Professor of Chemical Engineering

University of Texas

Austin, TX 78712-1062

Dr. Thomas Hess

University of Idaho

Department of Agricultural Engineering

Moscow, ID 83843

Dr. Jeffrey Hoke

Staff Chemist, Corporate Research

Engelhard Corporation

101 Wood Avenue

Iselin, NJ 08830-0770

Mr. Gus Hutchinson

Solar Kinetics Inc.

10635 King Williams Drive

Dallas, Texas 75220

Mr. Avtar Jassal

ES\&H Project Manager

SEMATECH

.2706 Montopolis Drive

Austin, TX 78741

Dr. Mark Jones

DOW

1776 Building

Midland, Michigan 48674

Ms. Deborah Kielian

City of Denver

Department of Health and Hospitals

Environmental Section

605 Bannock Street

Denver, CO 802044707

Mr. Nick Korte

Oak Ridge, Grand Junction

Oak Ridge National Lab

2597 B 3/4 Road

Grand Junction, CO 81503
Mr. Ephraim Heller

E. Heller \& Company

1311 Harbor Bay Parkway, Suite 1000

Alameda, CA 94501

Mr. Michael Hoffman

Business Development Manager

NEPCCO

2140- 100 N.E. 36th Ave.

Ocala. FL 34470

Mr. Bennett Howell

Solar Kinetics, Inc.

P.O. Box 540636

Dallas, TX 75354

Dr. Bruce Hutton

University of Denver

College of Business Administration

2020 South Race Street

Denver, CO 80208

Mr. Ohad Jehassi

Economist

U.S. EPA

Office of Toxic Substances

401 M Street, S.W. (TS-779)

Washington, DC 20460

Dr. Prashant V. Kamet

University of Notre Dame

Radiation Laboratory

Notre Dame, IN 46556-0579

Mr. J. R. Kittrell

Resident

KSE Inc.

P. 0. Box 368

Amherst, MA 01004

Dr. Carl Koval

University of Colorado

Department of Chemistry

Boulder, CO 80309-0215 
Mr. Raymond Krishock

Corporate Staff Engineer

E/M Corporation

P.O. Box 2400

West Lafayette, IN 47906

Ms. Linda Ladas

Solar Energy Industries Association

777 North Capitol St. NE

Suite 805

Washington D.C. 20002-4226

Dr. C.C. Lee

Risk Reduction Engineering Laboratory

U.S. Environmental Protection Agency

26 West Martin L. King Drive

Cincinnati, OH 45268

Ms. Norma Lewis

U.S. EPA

Emerging Technology Section

Cincinnatti, OH 45268

Dr. Noam Lior

University of Pennsylvania

Dept. of Mech. Engineering

297 TB/6315

Philadelphia, PA 19104-6315

\author{
Dr. Sixto Malato \\ CIEMAT-PSA \\ Aptdo.22 \\ E-04200 Tabernas (Almeria) \\ SPAIN
}

Mr. Keith Mason

Senior Economic Analyst

U.S. EPA

Office of Air and Radiation

401 M Street, S.W. (6103)

Washington, D.C. 20460

Dr. Augustus McEvoy

Institut de Chemic Physique

EPFL

1015 Lav sanne

SWITZERLAND
Dr. Peter Kuhn

Paul Scherrer Institute

CH-5232 Villigen

SWITZERLAND

Mr. Lawrence Laitres

Lawter Associates

157 Hillcrest Rd.

Fairfield, CT 06430

Ms. Gretchen Leslie

Technical Librarian

Washington State Energy Office

P.O. Box 43171

925 Plum Street S.E., Bldg. \#4

Olympia, WA 98504-3 171

Dr. Norman Lichtin

Boston University

Chemistry Department

590 Commonwealth Avenue

Boston, MA 02215

Mr. Douglas P. Lorriman

Chairman

Solarchem Environmental Systems

130 Royal Crest Court

Markhcem, Ontario L3ROA1

CANADA

Dr. Blair Martin

Air and Energy Engineering Research Laboratory

U.S. Environmental Protection Agency

Research Triangle Park, NC 27711

Mr. Ken May

Industrial Solar Technology

4420 McIntyre Street

Golden, CO 80403

Dr. Thomas McKinnon

Colorado School of Mines

Department of Chemical Engineering

Golden, CO 80401
Dr. Charles Kutal

University of Georgia

Department of Chemistry

Athens, GA 30602-2556

Mr. Jack Lauber

New York State Dept. of Environmental

Conservation

Division of Air Resources

50 Wolf Road

Albany, NY 12233-3254

Dr. Miriam Lev-On

Senior Consultant, Environmental

Protection

ARC0

515 South Flower Street

Los Angeles, CA 90071

Mr. Clovis Linkous

Research Engineer

Florida Solar Energy Center

300 SR 401

Cape Canaveral, FL 32920

Dr. John Malanchuk

IT Corporation

Regional Office

312 Directors Drive

Knoxville, TN 37923-4799

\section{Dr. Paul Martino}

American Petroleum Institute

1220 L. Street, NW

Washington, DC 20005

Ms. Naydene Maykut

Senior Air Quality Scientist

Puget Sound Air Pollution Control

Agency

110 Union Street, Suite 500

Seattle, Washington 98101 - 1038

\section{Mr. Derek McManus}

Wheelabrator Clean Air Systems

1501 E. Woodfield Rd., Suite200 West

Schaumburg, IL 60173 
Mr. Richard Miller

IT Corporation

312 Directors Drive

Knoxville, TN 37923

\section{Mr. Terrell Minger}

Center for Resource Management

1410 Grant Street, Suite C307

Denver, CO 80203-1 846

Mr. Nazim Muradov

Research Associate

Florida Solar Energy Center

301 State Road

Cape Canaveral, FL

Dr. J.H. Nelson

University of Nevada at Reno

Department of Chemistry

Reno, NV 89557

Dr. Manfred Noack

Senior Consulting Chemist

350 Knotter Drive

P.O. Box 586

Cheshire, CT 06410-0586

Dr. David Ollis

Chemical Engineering Department

N.C. State University

Box 7905

Raleigh, NC 27695-7905

Dr. Arijit Pakrasi

Senior Project Engineer

IT Corporation

312 Director's Drive

Knoxville, TN 37923

\section{Mr. Tom Paul}

Aegis Environmental, Inc.

2470 Wrondel Way

Suite 208

Reno, NV 89502
Mr. Steve Miller

Jacobs Engineering

175 Freedom Blvd

Kevil, KY 42053

Mr. Lawrence Mireles

2063 A San Antonio Ave

Alameda, CA 94501

Ms. Maria Murray

INRAD, Inc

181 La Grand Ave

Northvale, NJ 07647

Mr. Timothy Nicely

Solutions International

19528 Ventura Blvd., Suite 278

Tarzana, CA 91356

Dr. Richard Noble

University of Colorado, Boulder

Dept of Chemical Engineering

Engineering Center, CH 1-60

Campus Box 424

Boulder, CO 80309-0424

Mr. Tim O'Neill

Measurement Technologies Northwest

3822 Latone Lane, NE

Seattle, WA 98105

Dr. Bill Parker

Editor

Solar Progress

Box 175

North Perth 6006

AUSTRALIA

Mr. Chris Paulson

Friedlob Sanderson Raskin Paulson \&

Tourtillott

1400 Glenarm Place

Denver, CO 80202-5099
Dr. Emil Milosavljevic

University of Nevada, Reno

Mackay School of Mines

Reno, NV 89557

Dr. Booker Morey

SRI International

333 Ravenswood Avenue

Menlo Park, CA 94025

Ms. Maria Nargiello

Degu ssa Corp

Applied Technology Pigment Group

Suite 110, 150 Springside Drive

Akron, $\mathrm{OH} 44333$

Dr. David E. Nicodem

Instituto de Química-UFRJ, CT

Bloco A, Ilha do Fundao, 21949-900

Rio de Janeiro, RJ

BRAZIL

Mr. Carlos Nunez

U.S. EPA

AEERL-OCT (MD-6 1)

Research Triangle Park, NC 27711

Dr. Kevin O'Shea

Florida International University

College of Arts \& Sciences

Miami, FL 33199

Mr. Gerald Parkinson

McGraw-Hill, Inc.

17432 Teachers Ave.

Irvine, CA 92714

Mr. Greg Peebles

American Energy Technologies, Inc. P.O. Box 1865

Green Cove Springs, FL 32043 
Dr. Phyllis Pei

Manager, ES\&H

SEMATECH

2706 Montopolis Drive

Austin, TX 78741-6499

\section{Mr. Keith Pettus}

Huck International, Inc.

Safety, Health and Environmental Affairs P.O. Box 19590

Irvine, CA 92713

Ms. Anne Polanski

Solar Energy Industries Association

777 North Capitol St. NE

Suite 805

Washington D.C. 20002-4226

\section{Mr. Steve Pratt}

Merrick Engineers \& Architects

10855 East Bethany Drive

P.O. Box 22026

Denver, CO 80222

Dr. Greg Raupp

Arizona State University

Dept of Chemical Bio \& Materials

Engineering

Tempe, AZ 85287-6006

Dr. Mary Clare Robbins

Assistant Professor

Univ. of Texas at El Paso

M\&I Engineering

College of Engineering

El Paso, Texas 79968-0521

Dr. Shabbar Saifee

Senior Renewable Energy Spec.

Virgin Islands Energy Office

81 Castle Coakley

Christiansted, St. Croix

U.S. Virgin Islands 00820

Ms. Linda Schneider

Trojan Industries

3020 Gore Rd.

London, Ontario N5V4T7

CANADA
Dr. Nicola Peill

California Institute of Technology

Environmental Engineering Science

Department

MC 138-78

Pasadena, CA 91125

Dr. Gary Peyton

Illinois State Water Survey

Office of Environmental Chemistry

1008 S. Mattis Ave

Champaign, IL 61820

Dr. Wade Ponder

U.S. EPA

Air and Energy Engineering Research

Laboratory

Research Triangle Park, NC 27711

Dr. Charles Quinlan

KSE, Inc.

P.O. Box 368

Amherst, MA 01004

Mr. John S. Reese

NEPCCO

2140 N.E. 36th Ave.

Ocala, FL 34470

Dr. Tim Rose

EIC Laboratories Inc.

Research Division

111 Downey St.

Norwood, MA 02062

Dr. Craig Saltiel

University of Florida

CASE

3950 RCA Blvd., Suite 5003

Palm Beach Garden, FL 33410

Dr. Judith Schreiber

Director, Bureau of Toxic Sub.

State of New York Dept. of Health

2 University Place, Rm. 240

Albany, NY 12203-3399
Dr. Ezio Pelizzetti

Institute di Chimica Fisica

Universita di Parma

43100 Parma

ITALY

Dr. Pierre Pichat

U.R.A. au C.N.R.S.

Ecole Central de Lyon

E.P. 163, 69131 Eculty Cedex

FRANCE

Dr. Michael Prairie

Chemical Engineer

Sandia National Laboratories

P.O. Box 5800, Division 6216

Albuquerque, NM 87185-0703

Dr. Krishnan Rajeshwar

University of Texas at Arlington

Dept of Chemistry \& Biochemistry

Box 19065

Arlington, TX 76019-0065

Mr. Mark Rinaman

Law Environmental

112 Town Park Drive

Kennesaw, GA 30144

Dr. Kathy Rowlen

University of Colorado

C.B. 215

Boulder, CO 80309

Dr. Peter Schaeffer

West Virginia University

Agricultural Services Building

Division of Resource Management

P.O. Box 6108

Morgantown, WV 26506-6 108

Dr. Jeffrey Sczechowski

California Polytechnic State University

Civil \& Environmental Engineering Dept

San Luis Obispo, CA 93407 
Mr. Richard Segrave-Daly

Small Business Ombudsman

Pennsylvania Department of Commerce

Fulton Bank Building, Suite 901

Harrisburg, PA 17101

\section{Mr. Nanjun Shetty}

Geraghty \& Miller

2840 Plaza Pl., Suite 350

Raleigh, NC 27613

Dr. Kurt Sisson

U.S. Dept. of Energy

NP-42 1 H-088

1000 Independence Ave. SW

Washington DC 20585

Mr. Mark Smith

Tyndall AFB

Air Force Civil Engineering

Support Agency

Tyndall AFB, FL 32403-6001

Mr. Stephen Snyder

Energy Management Division Chief

HQ, Fort Carson \& 4th Infantry Division

Attention: AFZC-ECM-EM

Fort Carson, CO 80913-5000

Mr. Stephen Stiles

Chemist

Energetic Materials Chemistry Division

Indian Head Division, NSWC

Indian Head, MD 20640-5035

Mr. Michael Swan

Vice Resident

Process Technologies, Inc.

910 Main Street

Boise, ID 83701-0476

Dr. Ali T-Raissi

Florida Solar Energy Center

300 State Road 401

Cape Canaveral, FL 32920
Mr. L.M. Seigel

PRC Environmental Management, Inc.

1505 PRC Drive, Suite 220

McLean, Virginia 22102

Dr. Raymond Sierka

University of Arizona

Dept of Civil Engineering and Engineering

Mechanics

Tucson, AZ 85721

Mr. David Skiles

The Denver Smart Places Project

605 Bannock Street, Room 333

Denver, CO 802044707

Dr. Russell Smith

University of Texas at Arlington

Department of Chemistry

P. 0. Box 19065

Arlington, TX 76019

Dr. Herbert Spencer

EC\&C Technologies

4234 Chevy Chase Dr.

Lacanada, CA 91011

Mr. Jack Sullivan

Research Chemist

Tennessee Valley Authority

P.O. Box 1010

Muscle Shoals, AL 35660

Mr. Bob Sweeney

New Mexico Environmental Dept.

Groundwater Bureau

Harold Runnels Building

1190 St. Francis Dr.

Sante Fe, NM 87502

Mr. Hal Taback

Hal Taback Company

378 Paseo Sonrisa

Walnut, CA 91789
Dr. Nick Serpone

Department of Chemistry

Concordia University

1455 DeMaisonneuve Blvd. West

Montreal, Quebec H3G1M8

CANADA

\section{Dr. Subbas Sikdar}

EPA

Risk Reduction Engrg Lab

26 W. Martin Luther King Drive

Cincinnati, OH 45268

Mr. Jake Smith

Hennepin County Environmental

Management

417 North Fifth Street

Minneapolis, MN 55401-1309

Dr. Wayde Smith

UTRC - M.S. 30

Silver Lane

East Hartford, CT 06108

Dr. Donald Stedman

University of Denver

Department of Chemistry

2101 East Wesley Avenue

Denver, CO 80208

Dr. Ken-ichirou Suzuki

Toyota Central R\&D Labs

41-1 Yokomichi, Nagakute

Nagakute-cho, Aichi-gun

Aichi-ken 480- 11

JAPAN

Ms. Denise Swink

U.S. Dept. of Energy

EE-20 6B-052

Office of Industrial Technologies 1000 Independence Ave. SW

Washington DC 20585

Dr. Walter Tang

Florida International University

Dept of Civil and Environmental

Engineering

Miami, FL 33199 
Dr. Marion Thumauer Argonne National Lab 9700 S. Cass Ave.

Argonne, IL 60439-4815

Dr. Craig Tyner

Manager

Sandia National Laboratories

Solar Thermal Technology Department

Albuquerque, NM 87185-0703

Ms. Rebecca Vories

Infinite Energy

P.O. Box 481905

Denver, CO 80248

Mr. Frank Wilkins

U.S. Dept. of Energy

EE-222 5G-067

1000 Independence Ave. SW

Washington DC 20585

Dr. John Yates, Jr.

University of Pittsburgh

Department of Chemistry

Chevron 234

Pittsburgh, PA 15260

Dr. Mark Zappi

US Army Engineers

3909 Halls Ferry Rd.

Vicksburg, MI 39180-6199
Dr. Micha Tomkiewicz

Department of Physics

Brooklyn College, C.U.N.Y.

Brooklyn, NY 11210

Mr. John Vig

Army Research Lab

AMSRL-EP-ME

Ft. Monmouth, NJ 07703-5601

Dr. Richard Watts

Washington State University

Department of Civil Engineering

Pullman, WA 991642910

Ms. Suzanne Wilson

Environment and Safety Division

Anaheim Public Utilities Dept.

201 South Anaheim Blvd., Room 1102

Anaheim, CA 92805

Professor Courtney Young

219 ELC Building

Dept. of Metallurgical Engineering

Montana Tech.

Butte, MT 59701

Dr. R.G. Zepp

U.S. EPA

960 College Station Road

Athens, GA 30613-7799
Mr. Ronald J. Turner

Regional Manager

Kleinfelder

3249 East Harbour Drive

Phoenix, AZ 850347227

Dr. Darryl Von Lehmden

Midwest Research Institute

401 Harrison Oaks Blvd

Cary, NC 27513

\section{Mr. David Wickham}

TDA Research

12421 West 49th Ave

Wheatridge, CO 80033

Dr. Aaron Wold

Brown University

Department of Chemistry

Providence, RI 02912

Dr. Po Lock Yue

Hong Kong University of Science \&

Technology

Room 457 1, Academic Building

Clear Water Bay

Kowloon, HONG KONG 


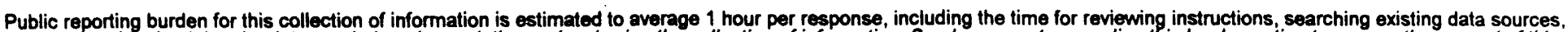

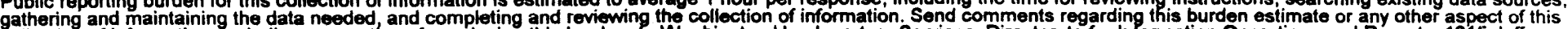

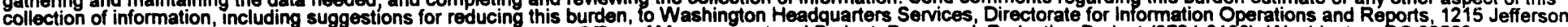
Davis Highway, Suite 1204, Arlington, VA 22202-4302, and to the Office of Management and Budget, Paperwork Reduction Project (0704-0188), Washington, DC 20503.

\begin{tabular}{|l|c|c|}
\hline 1. AGENCY USE ONLY (Leave blank) & $\begin{array}{c}\text { 2. REPORT DATE } \\
\text { October } 1995\end{array}$ & $\begin{array}{c}\text { 3. REPORT TYPE AND DATES COVERED } \\
\text { Technical Report }\end{array}$ \\
\hline
\end{tabular}

\section{TITLE AND SUBTITLE}

Bibliography of Work on the Heterogeneous Photocatalytic Removal of Hazardous Compounds from Water and Air, Update Number 1, to June, 1995

5. FUNDING NUMBERS

(TA) $S 151.3020$

\section{AUTHOR(S)}

Dan Blake

7. PERFORMING ORGANIZATION NAME(S) AND ADDRESS(ES)

National Renewable Energy laboratory

1617 Cole Boulevard

Golden, CO 80401-3393

8. PERFORMING ORGANIZATION

REPORT NUMBER

NRELITP-47320300

9. SPONSORING/MONITORING AGENCY NAME(S) AND ADDRESS(ES)

National Renewable Energy Laboratory

1617 Cole Boulevard

Golden, CO $80401-3393$

10. SPONSORING/MONITORING AGENCY REPORT NUMBER

NA

\section{SUPPLEMENTARY NOTES}

\section{2a. DISTRIBUTION/AVAILABILITY STATEMENT}

National Technical Information Service

U.S. Department of Commerce

5285 Port Royal Road

Springfield, VA 22161 12b. DISTRIBUTION CODE

UC-1404

\section{ABSTRACT (Maximum 200 words)}

This report is an update of a bibliography, published in May, 1994, of research performed on the photocatalytic oxidation of organic or inorganic compounds in air or water and on the photocatalytic reduction of metal-containing ions in water. The general focus of the research is on removing hazardous contaminants from air or water to meet environmental or health regulations. The processes covered are based on the application of heterogeneous photocatalysts. The current state-of-thsart in catalysts are forms of titanium dioxide or modifications of titanium dioxide, but work on other heterogeneous catalysts is also included in this compilation. This update contains 574 references, most published between January, 1993 and June, 1995, but some references are from earlier work that were not included in the previous report.

\section{SUBJECT TERMS}

adsorbent, adsorption, anion inhibition, aqueous phase, biotreatment, catalyst characterization, catalyst reactivation, disinfection, gas phase $I A Q$, immobilized $\mathrm{TiO}_{2}$, metal removal, metallized $\mathrm{TiO}_{2}$, modified $\mathrm{TiO}_{2}$, nonaqueous, photocatalysis, process efficiency, reductant, $\mathrm{TiO}_{2}$

\section{NUMBER OF PAGES}

104

16. PRICE CODE

A06

\section{SECURITY CLASSIFICATION} OF REPORT

\author{
19. SECURITY CLASSIFICATION \\ I OF ABSTRACT
}

20. LIMITATION OF ABSTRACT 
